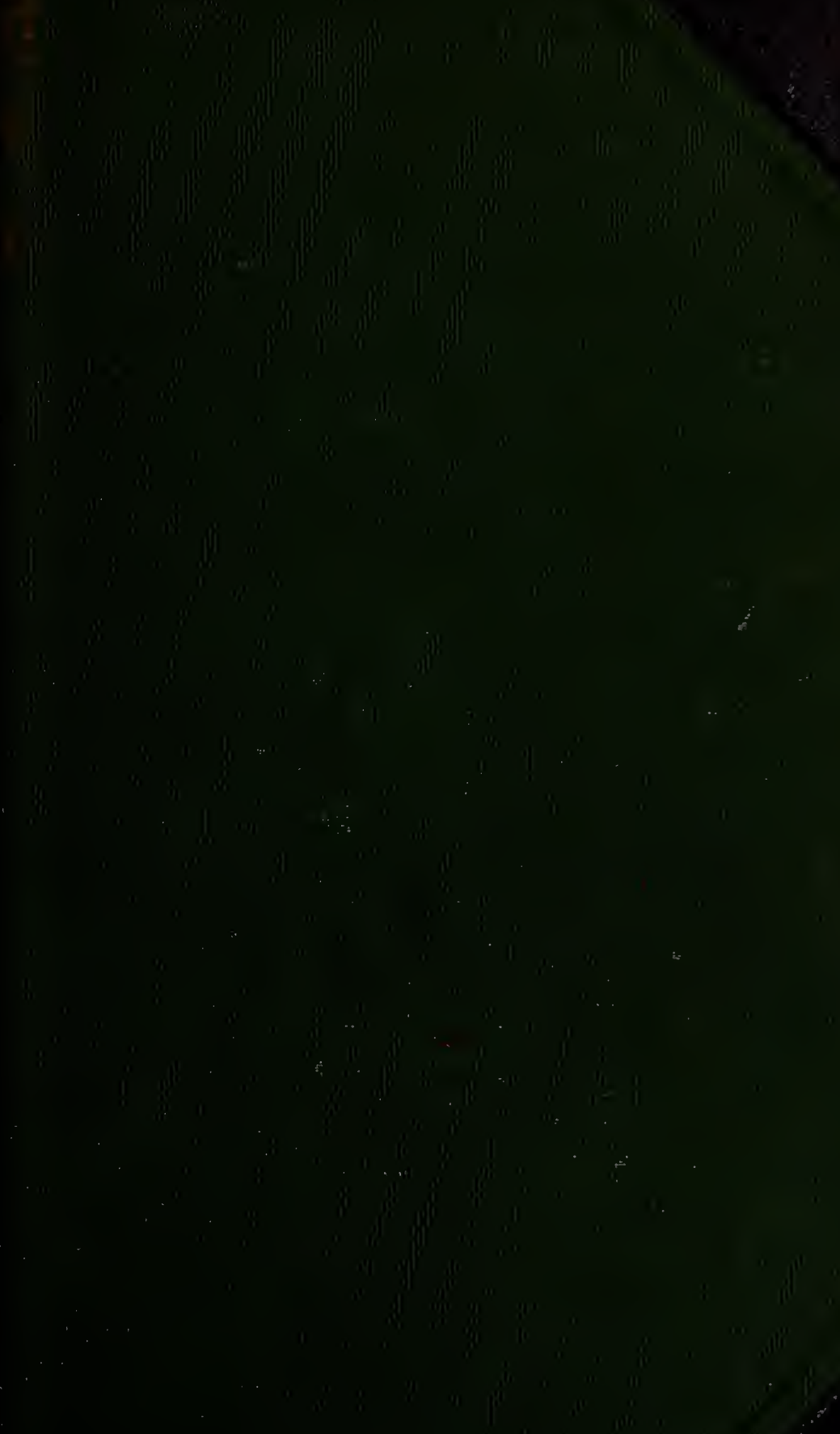



Return this book on or before the Latest Date stamped below. A charge is made on all overdue books.

University of Illinois Library

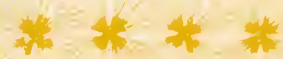

the of a th

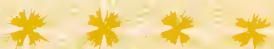

* $x^{2}-4 x$

HAPR 1? 1948

$v^{2}=2 y$

MAP - 3105

wath

-

DEC 241969

$y^{2}=3 x^{2}=y^{4}=3 x$

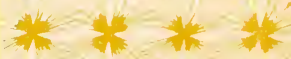

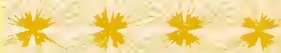

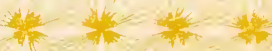

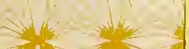

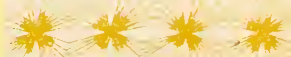

$x^{4}+x^{2}+1$

foc $x+4=$

1.

a

$\sqrt{2}+4 x+4$

the $1 / 4-24$

th $y=0$

कै

$\rightarrow-14$

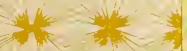

$-1 /=0$

$y=1$

MI32

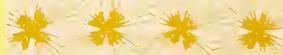

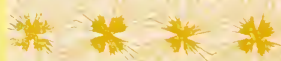

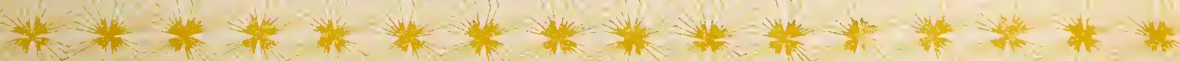

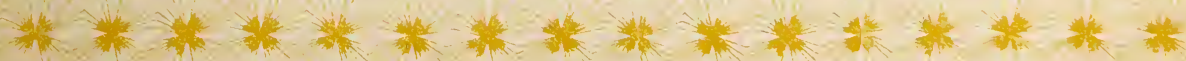

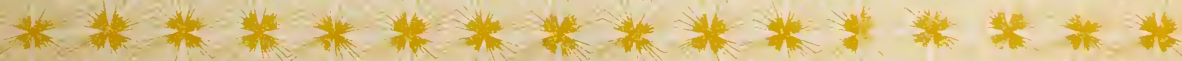



Digitized by the Internet Archive in 2016 


\title{
THEORY AND PRACTICE
}

OF

\section{VETERINARY MEDICINE}

NOTES TAKEN FROM

LECTURES

DELIVERED BY

A. H. BAKER, V. S .

PROFESSOR OF THEORY AND PRACTICE AT THE CHICAGO VETERINARY COLLRGE

\section{THIRD EDITION, REVISED}

\author{
CHICAGO \\ ALEXANDER EGER \\ PUBLISHER \\ 1911
}


COPYRIGHTED

AT WASHINGTON, D. C.

1011

BY ALEXANDER EGER 


$$
B 17+3
$$





\section{PREFACE TO THIRD EDITION.}

Owing to the rapid exhaustion of the second edition, a third edition of this book has been published.

While the style of arrangement has not been changed and additions are but few, still the book has been thoroughly revised and brought up to date.

The liberal patronage of the profession is herewith gratefully acknowledged.

A. E.

\section{PREFACE TO SECOND EDITION.}

The frequently expressed desire of Veterinary Students and Practitioners for a small and concise work on Veterinary Theory. and Practice has prompted me to publish this little book of Student's notes.

The similar edition issued several years ago was much appreciated by both students and practitioners to whom also I believe this new issue will be as welcome.

This being merely a book of student's notes the language used is somewhat free and non-technical, a fact that will, I hope, be excused by the critical reader.

A. E. 




\section{CONTENTS.}

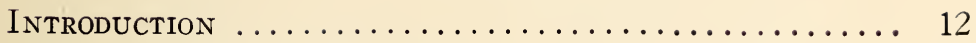

The Institutes of Medicine................... 13

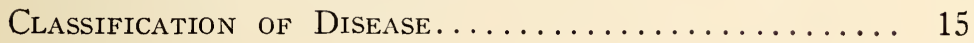

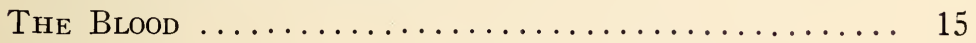

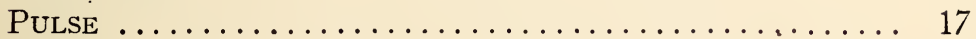

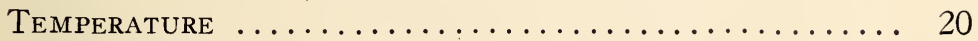

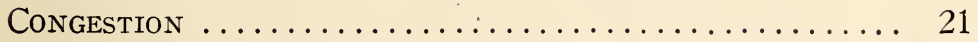

Results of Active Congestion.............. 24

Thrombosis ${ }^{\circ}$ and Embolism.................. 26

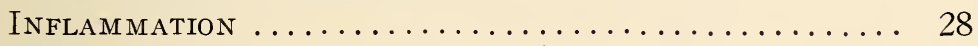

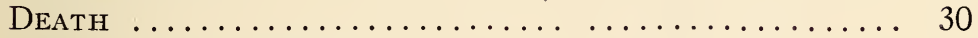

How to Kill a Horse.................... 33

Nosology .............................. 35

Rational Treatment of Disease in General........ 36

Rational Treatment of a Fever............... 37

Respiratory Diseases .................... 41

Rhinitis ..................... 42

Laryngitis ........................... 48

Chronic Laryngitis ................... 52

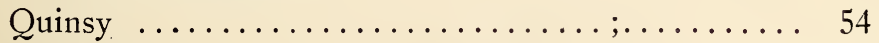

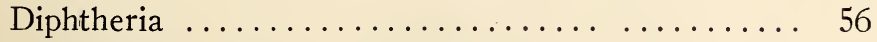

Bronchitis ........................... 57

Heaves or Pulmonary Emphysema........... 63

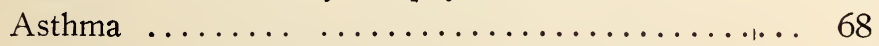

Pulmonary Congestion ................. 69

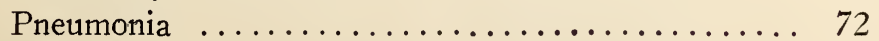

Pleurisy .............................. 81

Record of a Case of Pneumonia............ 88 
Diseases of the Digestive System.............. 89

Anatomy and Physiology................. 89

Phenomena of the Digestive Organs............ 91

Diseases of the Mouth................. 92

Congestion of the muccal membrane....... 92

Stomatitis .................. 93

Glossitis ...................... 94

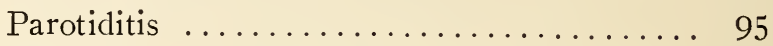

Ptyalism ......................... 96

Salivary Fistula ................. 97

Salivary Calculi ................... 98

Diseases of the Throat.................. 99

Pharyngitis ...................... 99

Diseases of the Oesophagus................ 100

Oesophagitis ....................... 100

Oesophagismus ................... 101

Chocking ..................... 101

Organic Diseases of the Oesophagus........ 104

Diseases of the Stomach.................. 105

Vomition ........................ 105

Indigestion $\ldots \ldots \ldots \ldots \ldots \ldots \ldots \ldots$

Acute Gastric Indigestion.............. 109

Chronic Gastric Indigestion............. 110

Gastritis ....................... 113

Chronic Gastritis ................. 115

Rupture of the Stomach............ 116

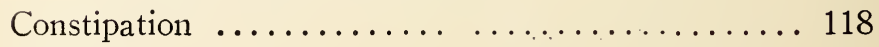

Diarrhœa ......................... 123

Acute Diarrhoa ......................126

Chronic Diarrhœa ................. 126

Colic .............................. 129

Flatulent Colic .................... 132

Intestinal Obstructions .............. 135

Eversion of the Rectum................ 137

Hæmorrhoids ...................... 139

Rupture of the Intestinal Wall. ........... 140

Enteritis ...................... 140 

Peritonitis ...................... 143

Dysentery ........................ 144

Diseases of the Abdominal Glands............. 145

Liver-Spleen-Pancreas ................ 145

Congestion of the Liver.............. 146

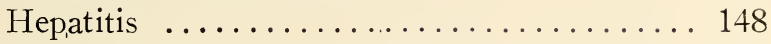

Chronic Hepatitis ................... 149

Fatty Degeneration ................ 150

Icterus $\ldots \ldots \ldots \ldots \ldots \ldots \ldots \ldots \ldots$

Constitutional or Blood Diseases............. 153

Variola ............................. 154

Anthrax ......................... 155

Rabies ............................. 158

Glanders ........................... 161

Strangles ........................ 170

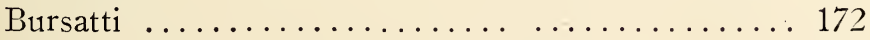

Botryomycosis ...................... 174

Corn Stalk Disease...................... 174

Surra .......................... 174

Influenza ........................ 175

Purpura Hæmorrhagica ................. 177

Scarlatina ............................ 179

Infectious Anemia ..................... 180

Rheumatism ..................... 182

Lymphangitis ....................... 186

Epizootic Lymphangitis . . . . . . . . . . . . . 189

Azoturia ............................. 190

Diseases of the Circulatory System............... 195

Palpitation .......................... 195

Cyanosis ............................. 197

Syncope ................................. 198

Acute Inflammatory Diseases. . . . . . . . . . . . . . . 198

Pericarditis ........................ 198

Endocarditis .................... 200

Myocarditis ...................... 203

Hypertrophy and Atrophy................. 203

Fatty Degeneration ................... 205 
Polypi or Tumors of the Heart............. 206

Rupture .......................... 206

Diseases of the Blood Vessels............... 206

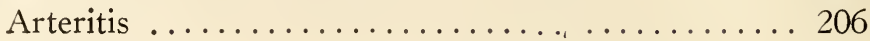

Aneurism ......................... 207

Phlebitis .......................... 207

Varix .............................. 208

Diseases of the Urinary System............... 208

Albuminuria ....................... 210

Hematuria ........................ 211

Diabetes Insipidus .................... 212

Diabetes Mellitus ..................... 213

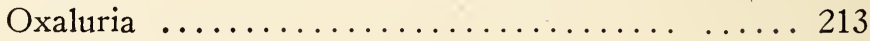

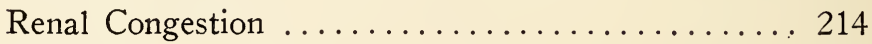

Nephritis ......................... 215

Renal Calculus ........................ 217

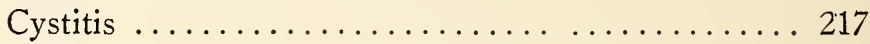

Dysuria .......................... 218

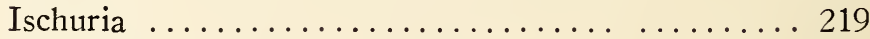

Enuresis .......................... 220

Urethritis .......................... 221

Tumors ......................... 221

Cysts ........................... 225

Diseases of the Bones.................... 228

Atrophy of Bone.................... 231

Constitutional Osteoporosis ............... 231

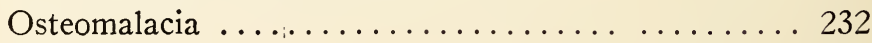

Rachitis ........................... 233

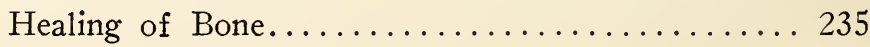

Diseases of the Nervous System.............. 236

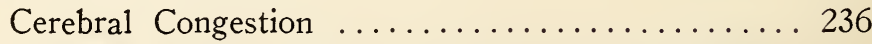

Cerebral Anæmia, Embolus, Apoplexy and Meningitis 237

Cerebral Softening .................... 239

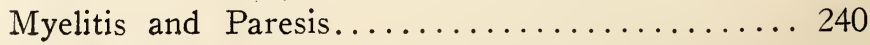

Hydrocephalus ....................... 241

Tabes Dorsalis ........................... 242 

Tetanus ........................... 243

Chorea ............................. 246

Diseases of the Reproductive System............ 247

Simple Pox ....................... 247

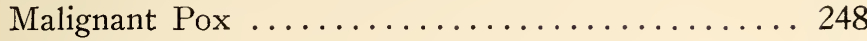

Urethritis ......................... 249

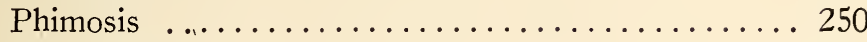

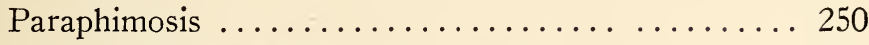

Dropsy of the Ovaries.................... 251

Hydrometra ........................ 251

Nymphomania, Hysteria ................. 252

Leucorrhøa ....................... 252

Onanism ............................ 253

Diseases of the Organs of Special Sense.......... 253

Simple Ophthalmia .................... 253

Hæmatoides Fungus ...................... 254

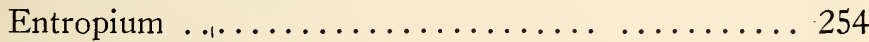

Ectropium, Leucoma .................. 255

Ulceration of the Cornea................ 255

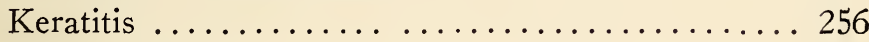

Filaria Oculi .....................256

Amaurosis .......................... 257

Detachment of the Retina................ 258

Staphyloma ........................ 258

Periodic Ophthalmia .................... 259

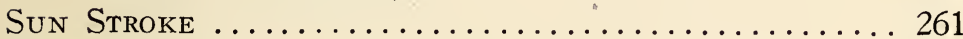

DeAth вy Lightning.......................... 264 


\section{INTRODUCTION.}

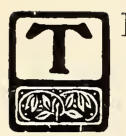

HE PRACTICE OF VETERINARY MEDICINE is both a science and an art. As a science it inquires into the existence, conditions, nature and causes of disease. A science relates to facts; it is not theory, it can be demonstrated. The existence, conditions, and nature of the case, the location of the disease, the causes of it,- these are all facts.

As an art Veterinary Medicine directs its efforts toward the prevention, treatment, and recognition of disease. It used to be considered as an art only; but now, the world over, it is regarded as a scientific profession.

What is disease? Disease is any deviation from the normal condition i. e., from health. Health is best and most concisely defined as the condition in which all the vital organs perform their functions rhythmically. A disorder in one organ upsets the others. Health implies that all are working in harmony. 



\section{THE INSTITUTES OF MEDICINE.}

The Institutes of Medicine are necessary in order to facilitate the study of disease.

Disease is either functional or organic. A functional disease is one in which the physiological action is disturbed, but does not show pathological lesions or morbid anatomy. A disease is organic when it gives indication of morbid anatomy. A disease may be both functional and organic; but it may be purely functional without showing any change of structure, the physiological action alone being disturbed. Hence a good knowledge of physiology and anatomy is necessary in order to understand disease, --physiology for the functional diseases and anatomy and histology for the organic.

Pathology is the doctrine of disease, i. e., it is the doctrine of everything connected with morbid conditions, either functional or organic. It is divided into:

1. General Pathology.

2. Special Pathology.

General Pathology treats of diseased conditions in general without reference to any special case. Special Pathology takes into consideration special diseases and treats of their morbid anatomy.

Under general pathology the following factors come into consideration:

1. Etiology-Cause.

2. Symptomatology - Semeiology, Symptoms.

3. Pathogeny-Pathogenesis (the generation, production and development of disease).

4. Nosology-Classification and nomenclature. 
5. Diagnosis-Recognition of the disease.

6. Prognosis-Probable termination.

7. Morbid Anatomy-Structural changes produced by the disease.

8. Hygiene-Prevention of disease by good management.

9. Therapeutics-Medicinal indications.

Etiology.-The causes of disease are divided into two classes, predisposing and exciting. The predisposing arise within the body,- they are intrinsic. Conformation and heredity are examples. Consider a case of purpura hemorrhagica, a specific disease due to the action of some microbe on the red blood corpuscles. This germ lives especially in dirty stables, but is resisted by animals in good health; let the animals become debilitated, however, and microbian invasion occurs. The debilitation may be caused by a decayed tooth, by poor digestion, etc., and we call these predisposing causes. A narrow chest and straight ribs predispose an animal to chest diseases; a sickle hock to curb; a straight hock to bone spavin.

The exciting causes of disease in the horse are:-

1. Overwork or too little work.

2. Exposure to extreme changes of temperature and to other meteorological conditions.

3. Indigestible and impure food.

4. Poisons, foul drinking water and contagions.

Symptomatology.-The symptoms of disease are the signs, changes, actions, inclinations, and feelings expressed by the animal. It covers everything that can be noticed as differing from the normal. In order for a practitioner to be able to recognize these, he must first be familiar with the normal habits of the various species of animals. For everything differing from the normal is a symptom of disease.

Pathogeny. - The generation of disease relates to its minute cause, for example a bacterium; the production indicates how the disease is produced by the cause; the development is the 


result of the action of the cause. Tuberculosis is produced by the multiplication of the bacillus of tuberculosis, and this bacillus causes the disease; caseation, cell-infiltration, and miliary tubercles are the result of the germ action.

Diagnosis.-To be able to diagnose is to succeed, either in veterinary practice or in the human. Next comes logical reasoning power-to be able to put the symptoms together. The veterinarian cannot ask his patient anything, and he must arrive at his own conclusions from what he sees.

Hygiene.-This relates to diet, ventilation, drainage and general management (housing, blanketing, feeding and exercise). Seventy-five per cent of one's practice is produced by the bad management by the owners of animals.

\section{CLASSIFICATION OF DISEASES.}

Diseases are divided into three classes, Epizootic, Enzootic, and Sporadic. An epizootic disease attacks large numbers of animals over a large tract of country; there is no local cause. Any specific disease may become epizootic, as influenza in horses. An enzootic disease is due to a local cause and it may also attack large numbers over a large tract of country. Specific diseases are at times enzootic; glanders might be or rabies. A sporadic disease is one which occurs in single scattered cases without specificity. Diseases of this kind are non-contagious diseases.

\section{THE BLOOD.}

Arterial blood is scarlet; venous blood is blue; dead blood is black. Under the microscope blood is nearly colorless. It is made up of water and salts and the red and white blood corpuscles. It also contains fibrin forming elements; fibrin itself does not appear in the circulating blood. The function of the blood is to carry nutrition. If the circulation is disturbed a disturbance of the nutrition will occur. Some parts of the body suffer more from interrupted nutrition than others, the brain for 
instance. The amount of intelligence in an animal depends largely upon the amount and character of the brain circulation.

The composition of the blood is as follows:

Water ..........784 parts to 1000 .
Corpuscles .......
Albumin, salines and
$\quad$ parts to 1000
Fibrin .......... 2.2 parts to 1000

Any material change in the blood either produces disease or is the result of disease. If the solids increase the water decreases; if the solids decrease the water increases. An excess of solids and a decreased amount of water is designated as Plethora. Anaemia is the condition in which there is a decrease in the solids and an increase in the water. A plethoric animal is said to have very rich blood; the blood vessels are increased in size, the pulse is stronger and the mucous membranes are brighter. Plethora disposes the horse to certain diseases as AZOTURIA for instance, and ACUTE PLETHORIC LYMPHANGITIS. In these diseases there is an excess of the solids in the blood at the expense of the water. Plethora itself, however, does not constitute a disease.

- The symptoms of ANAEMia are:

Paleness of mucous membranes.

Decrease in the size of the blood vessels.

Decrease in the strength of the pulse.

General debility.

Liberal feeding, especially of nitrogenous food and moderate work are the causes of plethora. Debilitating diseases, chronic indigestion, and poor food, and certain parasites cause anæmia of the body:

Physiology teaches that fibrin is decreased in certain animals and they bleed easily. Such a conditicn is called Hemophilia, where an excessive hemorrhage will occur from a small wound. For example, a colt sweating blood is a case of hemophilia. Cases of castration occur in which the colts are disposed to bleed. Cats have more fibrin in their blood than any other animal. It is said that you cannot bleed a cat to death. 

Fibrin is also decreased in certain diseases. In pregnancy there is a decreased amount of fibrin. Some idiosyncracy may be a factor in causing a lack of fibrin in the blood. The function of fibrin is to prevent hemorrhage; if it were not for fibrin every animal would bleed to death.

Anæmic animals are lacking in fibrin; animals starving to death are an exception. The fibrin in their blood is increased. A local blister increases the fibrin in the adjacent tissues. During debilitating diseases as in purpura hemorrhagica, the fibrin is decreased.

\section{PULSE.}

To define the pulse concisely is difficult; the various dictionaries give a variety of definitions. Probably the American Pocket Dictionary has the best. According to it the pulse is the expansion and contraction of the arteries as the result of the action of the heart. The latter part of the definition is necessary for the vessels are dependent upon the action of the heart.

Arteries have three coats, the middle one, which consists of non-striated muscle, the inner one, the intima, and the outer one oi the adventitia. The muscle is controlled by the vas.o-motor system, which maintains the tonicity of the vessels. The vasomotor system is dependent upon the general condition of the body for its vitality.

The pulse depends upon the tonicity of the arterial muscles.

These muscles depend upon the vaso-motor system.

The vaso-motor system depends upon the general condition.

A horse might appear to be in apparently good health but if his pulse runs up to 60 or drops a beat now and again, then something is wrong with him. The pulse is the index to the nervous system.

The Effect of an Acute Inflammatory Disease Upon the Pulse.-In the first stage the nervous system is excited; the pulse is strong, full, regular and increased in frequency a little. In the second stage the nervous system is still more excited, but the pulse is growing weaker. Under such a condition the pulse is rapid, smaller and weaker than normal. In the third stage, 
supposing it to be fatal, the general system is becoming exhausted, and the pulse keeps growing weaker until finally it becomes imperceptible, the heart ceases to beat and the animal is dead.

The pulse of the horse varies from $35-45$ beats per minute. Under different conditions, on account of some idiosyncrasy, or external influences such as heat, cold, exercise, etc., the pulse of the horse may be faster or slower than 35 . In taking the pulse approach the horse slowly and quietly, and in an unconcerned manner place one hand high up on his nose to steady the head. The right hand is the best to use. Let the other feel for the sub-maxillary artery. Roll the artery with the fingers making sure of its location, and then count the pulse. Give the animal time to quite down, else the first count will be too high, due to the excitement of your approach. Do not look the horse in the eye. It makes him nervous.

The pulse of the ox runs from 45 to 50 , with an average of 45 . The pulse of the sheep runs from 70 to 80 , with an average of 72 or 75 . The pulse of the $\operatorname{dog}$ is 72 , the same as in the human.

In the ox it is not best to take the pulse from the submaxillary artery, for the skin over that region is thick, loose and pendulous, and this makes it difficult to find the pulse beat or if found to determine its correct character. The carotid artery in the neck is more suitable. Work the fingers under the margin of the levator humeri and feel for the artery. In the sheep, if the wool extends far down on the legs, then listen to the heart.

Of the various kinds of pulse we have the following:

1. Frequent and infrequentreferring to the number of beats per minute. Physiological conditions and disease cause the pulse to vary.

2. $Q \mathrm{uick}$ and slow-referring to the actual time of each individual beat.

3. Large and sma11-with reference to the volume of blood making up the pulse wave. 



\section{4. $\mathrm{Hard}$ a $\mathrm{nd}$ s of $\mathrm{t}$-this characteristic} measures the compressibility of the artery.

When the pulse gets very small and hard, it is said to be wiry-the artery feels like a wire-or thready, as it is sometimes called. In inflammatory diseases of mucous membranes the pulse is not as wiry as in the inflammation of serous membranes. A pulse is soft because it is more compressible than normal. This is due to prostration of the nervous system. The softest pulse we ever meet with is that of acute bronchitis-a very light pressure will cut it off entirely.

The dicrotic pulse is one composed of two beats, both of which correspond to one beat of the heart. The two beats are quite distinguishable to the touch. The phenomenon is only an exaggeration of what occurs in the normal pulse. After each systole the volume of blood in the aorta recoils, thus developing a new positive wave, the dicrotic wave or recoil wave. In certain fevers, when the pulse is soft, this secondary wave can be felt. The best illustration of a dicrotic pulse can be found in a case of azoturia three days old. In this condition there is not force enough in the left ventricle to empty itself and the systolic action is wavering. The natural effect of this is to produce passive congestion in the capillaries.

An irregular pulse is non-rhythmic in its action; it may be irregular in frequency, i. e., it may beat at 60 and then jump up to 80 or 90 , then drop back again. Or the pulse may be irregular in character; this is apt to occur when the force of the heart muscle is not equal to the work it has to do.

An intermittent pulse drops one or more beats or even part of a beat at intervals. The intermittency may be regular or irregular. Convalescent cases of pneumonia are apt to develop an intermittent pulse. The most common form is a drop of every fifth beat; an irregular intermittency may occur as a drop of every fifth, seventh and seventeenth beat, etc.

Venous Pulse.-This is seen in the jugular vein, due to weak cardiac action. It is produced by the blood flowing back into the jugular during the systolic action of the right side of the heart. In horses the jugular pulse always indicates a weak 
heart. In the ox and other ruminants we get the jugular pulse during rumination,--purely a physiological condition. -

The pulse is the most important means of making a diagnosis. One should become familiar with the various kinds of pulse. The sense of touch should be cultivated so as to be able to differentiate the characteristics of the pulse.

\section{TEMPERATURE.}

As regards the temperature of animals there are two conditions to be found; warm-blooded animals maintain an even temperature regardless of the surrounding medium. Coldblooded animals have the same temperature as the medium in which they live. All mammals are warm-blooded; amphibians, fishes and insects are cold-blooded.

In a warm-blooded animal any rise in temperature denotes a fever; any loss denotes waning vitality. The amount of the fever is measured by the number of degrees of rise in temperature; the subnormal temperature is measured by the number of degrees the temperature is lowered.

The normal temperature of the horse is 100 ; if the temperature rises above 104, the case is serious, and if above 106, dangerous. The temperature may rise to 107,109 , or possibly higher and recover if reduced promptly, i. e., in a few hours. But if a horse's temperature stands at 106 for a week he will die; at 107 for three days, or 109 for three hours, or 110 for one hour, a horse will die. A sub-normal temperature of 93 is always fatal in the horse. In fact 2 degrees below normal is very dangerous and nearly always fatal.

It is not safe to guess at the temperature, the practitioner must measure it with a clinical thermometer. The best place to take the temperature is at the anus, although there is some danger in inserting the thermometer. The animal may not like it and resist and kick. Therefore always stand to one side of the horse, he cannot kick out at the side. When inserting the thermometer in the anus, notice whether it is closed or not. In nervous prostration it is usually relaxed and the thermometer must be held in place. 

A sub-normal temperature may be produced artificially at a much lower point than it can occur in disease. The best records of this are in the human. Practitioner Peters took the temperature of a woman found drunk in the winter and it was 78.8; she recovered. This is the lowest case on record. It is said that an animal cooled to 75.2 will die.

The following is a table of the temperature of the domestic animals, taken from the German with the addition of Dr. Baker's :

$\begin{array}{lccl}\text { Animal. } & \text { No. of Observ. } & \text { German. } & \text { Baker. } \\ \text { Horse } & 78 & 99.9 & 100 \\ \text { Ox } & 16 & 103.4 & 101-2 \\ \text { Sheep } & 39 & 103.1 & 102-3 \\ \text { Dog } & 162 & 102.6 & 101-5 \\ \text { Hog } & 13 & 103.4 & 102 \text { (Brog.) } \\ \text { Fowls } & \ldots & 106.7-111 & 107-111\end{array}$

The temperature varies somewhat according to the atmospheric conditions and depends upon the amount of exercise to which the animal has been subjected. In taking the temperature the animal must be quiet, exercise raises the temperature. Driving animals raises their temperature, especially hogs, whose fat prevents them from cooling off.

In conducting the mallein test for glanders in horses, the temperature should be taken before feeding and drinking; cold water lowers the temperature. The animals should be comfortably placed so as not to cause them any excitement.

\section{CONGESTION.}

Congestion (Latin congere, to heap up) is defined as the increased flow of blood to a part. It is also called HYPERAEMIA, meaning an excess of blood. It is divided into Active and Passive. The active is confined to the arterial system, the passive to the venous. There is also another congestion which is called HYPOSTATIC. Congestion is described with the name of the organ affected; as, cerebral, hepatic, renal, etc.

A physiological congestion may be produced by nature to allow the organ to perform its function. For example, as par- 
turition approaches, the mammary glands become congested in order to stimulate the cells to secrete the milk. Otherwise agalactia would be the result.

The causes of active congestion are:

1. Increased blood pressure.

2. Diminished arterial resistance.

The first is due to an obstruction in one part causing an increased volume of blood to another, thus raising the pressure in the vessels. At first their walls remain practically of the same diameter, but later on become dilated by the increased volume of blood.

The second is caused by the nerves; the vaso-constrictors become depressed and as a result the arterioles dilate.

Whatever the irritating factor causing congestion, the immediate effect of it is a spasmodic contraction of the blood vessels. Dilatation then follows; the red blood corpuscles pile up in the enlarged lumen of the blood vessel, forming rouleaux, while the leucocytes take up their position along the vessel wall to which they apply themselves very closely. By means of their ameboid movements and on account of the increased pressure they work their way through the vessel into the surrounding tissues. At the same time a considerable amount of blood plasma has exuded into the tissues. The blood current has slowed down so that there is almost complete stasis, the blood is thicker and more viscid, and the red blood corpuscles may become pressed out into the tissues either by diapedesis or rhexis.

In hemorrhage by diapedesis the blood passes out into the tissues through the pores of the vessels,- the plasma, the leucocytes, and some few red blood corpuscles. In hemorrhage by rhexis the pressure is so great that a rupture occurs in the vessel wall, and the whole number of the blood elements passes through the rupture. As a result we get an extravasation of blood, a patch of tissue outside a vessel infiltrated with blood. Oedema is produced by hemorrhage by diapedesis.

If the congestion in the vessel is severe enough to plug it, an infarction may result. The stasis of blood in the vessel cuts off the nutrition from the surrounding tissues and they die. This is most apt to occur where there is no collateral circulation, 

Petechiæ are small red spots formed by the blood oozing out through the vessel. When a more extensive hemorrhage forms it is called ecchymosis. Hemophilia is an abnormal tendency to bleeding. It is usually hereditary.

After an excessive hemorrhage,-one that would produce infarction, gangrene is apt to follow. Moderate congestion maintained for any length of time, i. e., indefinitely, will result in hypertrophy. The opposite extreme, anæmia, will produce atrophy of a part.

The causes of passive congestion are:

1. Mechanical otstruction.

2. Diminished cardiac power.

3. Gravitation.

4. Altered conditions of the blood.

5. Defective secretion.

The last four of these causes we speak of as more complicated. The natural result of diminished cardiac.power is less force to drive the blood through the vessels. The venous system especially suffers. Passive congestion of the lungs is common.

Passive congestion by gravitation is seen in horses whose legs stock or swell up. The cause of this is a poor circulation in which the walls of the blood vessels are weak and the veins become dilated, and the blood settles in them by gravitation.

Altered conditions of the blood are seen especially in blackleg, in which the quarters swell up, forming cracklirg tumors. When one of these is punctured, bloody water flows out; the blood is tarry from the action of the germs causing the disease. The swollen quarters are passively congested.

- Defective excretions in an animal cause œdematous swellings. In other words, the emunctories are inactive. For instance, a horse's legs swell-he is otherwise in good health-give him a purgative and the œdema will disappear.

The topical signs of active congestion are as follows:

1. Redness-Rubor.

2. Enlargement and swelling of arterioles and thickening of their walls-Tumor.

3. Heat-Calor. 
4. Pain-Dolor.

5. Altered function of the affected organ.

The swelling in active congestion is resilient-press the part with the finger and the depression at once vanishes.

The topical signs of passive congestion are:

1. Swelling of an œdematous nature, i. e., doughy, inelastic; swollen tissue pits on pressure.

2. Congested area is sharply circumscribed.

3. Passive congestion tends to degeneration, never to inflammation.

In active congestion the swelling is resilient, it fades away imperceptibly into the healthy part; the congestion tends to run into inflammation on account of altered nutrition; the liquid oozing through the pores of the vessels is plastic and the swellirg is apt to become permanent; and it may produce death by gangrene.

In passive congestion the swelling is doughy, and is sharply circumscribed; the congestion tends to degeneration, never inflammation; the liquid oozing through the pores of the veins is not inclined to coagulate nor organize.

In passive congestion of the lungs an apparently paradoxical condition occurs because the blood leading to the lungs is venous, although carried by arteries; and that going away is arterial, but carried by veins. We call it passive congestion of the lungs when due to defective cardiac power, and active when due to diminished arterial resistence.

\section{RESULTS OF ACTIVE CONGESTION.}

When active congestion occurs in a part of considerable size and that in excess, moist gangrene results. Gangrene is of two kinds, moist and dry. Moist gangrene occurs in tissues whose nutrition has been suddenly cut off and which therefore dies. The dead part is moist gangrene. Dry gangrene is the result of starvation, that is, when the nutrition to a part is slowly cut off.

In moist gangrene the part is cold, insensible, and if punc- 

tured a moisture comes out. If occuring in white skin the color is black. In the horse the color of the gangrenous part is not so well contrasted. The dead part decomposes rapidly and sulphuretted hydrogen is produced, which is found in solution in the moisture of the decomposing mass, giving it the odor of rotten eggs, a most characteristic smell. A silver probe inserted in the tissue will at once become black. Between the living and the dead parts there is a red line of demarcation. If the gangrenous portion is small, the part may slough off, the separation taking place at the line of demarcation. By getting rid of the dead part the life of the animal is saved; but if the dead portion is too large to remove, absorption of the putrefactive matters takes place and poisons the blood and kills the animal. The dead part is usually spoken of as having a cadaverous smell.

In the time of the outbreak of contagious pleuro-pneumonia in 1887 , the disease was found to have existed for two years among the cows in the distillery sheds fed on distillery slops. Amongst these cows were many with bob-tails and it was said that the bull-dogs had bitten them off. But upon inquiry it was found that these animals had been inoculated against pleuropneumonia, and that a careless method of inoculation (serum probably decomposed) had set up a violent inflammation in their tails. These had become gangrenous and had sloughed off.

Moist gangrene often occurs in the lower lobe of the lung. The part cannot slough off and the animal dies from absorption of putrefactive products.

If moist gangrene occurs in a small part it is called NECROSIs.

In fistulous withers we speak of the dead parts removed as necrotic tissue.

Dry gangrene is death of tissue when the part dies from inanition, i. e., slowly starving to death. The part becomes paler and paler, losing its natural heat in proportion to the lack of nutrition.

The results of dry gangrene are:

1. Mummification-the part dries up, shrivels, and dies; it hangs on and will not drop off.

2. Chalky degeneration (calcareous)- 
most common in the human. In this condition the chalky portion crumbles off as in leprosy. It never occurs in horses.

A common example of dry gangrene may be seen in a sitfast on the horse. It is caused by the screws of the saddle extending too far down and producing pressure upon the skin; the part loses blood and dries, becoming gangrenous and insensible to pain. It does not slough, but finally produces a zone of inflammation around it. The only way to cure it is to remove the dead part and to remove the cause.

\section{THROMBOSIS AND EMBOLISM.}

A thrombus is a plug or clot in a vessel remaining at the point of formation. An embolus is a clot or other plug brought by the blood current from a distant vessel and forced into a smaller one, thereby obstructing the circulation.

Thrombosis is the coagulation of the blood within the heart or blood vessels during life-it is the plugging of a vessel by a thrombus. Embolism is produced by an embolus, which is a body floating in the blood until it lodges in a vessel too small for it to pass through. In either case active congestion would be produced by increased blood pressure. When a thrombus forms in a part which has no collateral circulation, the part dies from inanition. In the brain there is no free anastomosis between the vessels and if emboli lodge in them the tissues soon degenerate and die.

In embolism a clot has formed perhaps on the valve of the heart, or a little fibrin lodged among the chordæ tendineæ; a little piece floats off to some distant vessel and lodges, forming an embolism. An illustration of this may be seen in a horse which suddenly becomes lame; he drives all right for two or three miles and then suddenly goes lame on one leg, in a few minutes is practically on three legs. He sweats profusely and the affected leg is cold. After rest he is all right, but this experience occurs again and again until he is practically useless. The cause is an arterial embolus. During exercise the collateral circulation 
. 

of the leg is not sufficient and congestion occurs producing lameness. Severe exercise would have caused gangrene if the horse had not become so lame that he could not go any further.

An ante-mortem clot is light colored; a post-mortem clot is black. In making a post-mortem we often find in the cavities of the heart a clot resembling chicken fat, lodged among the valves, - a soft and friable mass. The remaining portions of the cavities are filled with black clots. The chicken fat clot is formed before death; it varies in color and density, from a soft yellow substance to a fibrinous grayish mass. The old antemortem clots are usually found in the posterior aorta or in the iliac arteries. They are more or less hard and somewhat elastic, rather filling the vessels. The degree of hardness indicates their time of formation. Very hard clots may have formed months back, even years perhaps. An ante-mortem clot consists of fibrin, leucocytes and some few red blood corpuscles, which fade out.

A post-mortem clot consists of all the elements of the blood and if the coagulation is rapid is of a dark red or black color.

An IRRITANT is anything that produces a local disturbance primarily and a constitutional disturbance secondarily. It is a substance which when applied to a part causes a greater or less degree of vascular excitement. The stimulating influence of an i: ritant may simply alter the function of the part, it may produce marked congestion and it may result in inflammation.

The effects of local irritants are usually the same as in active congestion,--redness, swelling, heat and pain. The topical signs are the same also.

It is the action of an irritant to stimulate, but the various irritants do this in different ways. For example, alcohol increases the function and circulatory action of the heart without irritating it; on the other hand turpentine irritates the part to which it is applied. If aromatic spirits of ammonia be given internally the mucous membrane of the stomach will be irritated and stimulated. If the dose is increased, it produces increased functional activity through the increased local irritation upon the mucous lining of the stomach.

The secondary or constitutional disturbance of an irritant 
is produced by reflex action. For example, the local irritation of a nail prick in the navicular bursa causes pain. This is transmitted to the brain by the efferent nerves, where this irritation may stimulate the efferent nerves, and as a result be carried to all parts of the body, causing an increase in the temperature, in the frequency and hardness of the pulse, and in the respiration. Eventually a general emaciation results. These effects may be mild or they may cause death, but they all come as the result of the action of some irritant.

\section{INFLAMMATION.}

A constitutional disturbance carried to the extreme always produces inflammation. According to Gould inflammation (Latin inflammare, to burn) is a condition of nutritive disturbance, characterized by hyperæmia, with the proliferation of the tissue cells, and attended by one or more symptoms of redness, swelling, heat, pain, discoloration and disordered function.

As a result the part may be very much discolored.

To appreciate an inflammation keep in mind active congestion; it is impossible to have an inflammation without active congestion, although we may have active congestion without inflammation. Viscidity of the fluids of the blood and tissues plays a1 important part. The increased fibrin causes bad results and aggravates inflammatory tendencies.

In inflammation the topical characteristics of active congestion are exaggerated, more pain, burning and throbbing. The proliferation of cells as the result of inflammation is seen in the healing of wounds. It has been said by some that inflammation cannot occur without cell proliferation, but I (Dr. Baker) say that it can; the process of inflammation may be going on for hours before any proliferation of cells takes place. But altered function is inseparable from inflammation; inflammation cannot occur without altered function.

Inflammation terminates by resolution when the inflammatory deposits soften, liquefy and resolve, leaving no traces of the disease behind. If any trace is left, it is not resolution. 


The stages of inflammation are three:

1. Hot dry stage.

2. Wet stage--.the stage of effusion.

3. Stage of suppuration, or formation of

pus.

The hot, burning stage is characterized by hyperæmia, exudation of plastic substances, infiltration of the surrounding tissues, discoloration of the tissues in the form of redness and suspension of function. This stage is short, an average of six hours.

In the wet stage, or stage of effusion, serum exudes from the blood vessels. The serum is thin, non-coagulable (not plastic); it infiltrates the surrounding tissues and produces œdema. This second stage is long, indefinite, lasting from a day or two to a month or more. It is called the wet stage from the effusion of serum. It is particularly illustated in the inflammation of mucous membranes.

The formation of pus in the third stage is brought about by the disorganization of tissue in which the inflammation occurs. The effusion of serum, pressure upon the nerves, and altered nutrition in a part result in a small focus of disorganized tissue, which eventually grows into a cavity containing pus. The pus-forming germs are most commonly the cocci (streptoand staphylo). These germs inoculated into healthy tissue will produce suppuration, but is it possible to produce a suppurative inflammation without them. Hamilton, the Scotch pathologist, demonstrates this as follows:

Sterilize the surface of a part, make an incision, and insert a sterile capsule containing a drop of croton oil. Seal over the wound with collodion and after three days you will find pus, but no germs. As soon as the capsule is dissolved, the croton oil cauterizes the part setting up an inflammation which runs the various stages until pus is formed.

Of the various terms used in describing inflammation, we have the following:

Induration-swelling hardens.

Adhesion-a growing together of two surfaces; seen especially in pleurisy, where the costal pleura adheres to the pulmonary pleura. 
Organization-the inflammatory deposits (the proliferating cells, coagulated albumen, fibrin, etc.) become permanent by the growth of the connective tissue cells, new formation of blood vessels, and the penetration of new nerves, the previous seat of inflammation becoming a permanent part of the animal.

Disorganization-a degeneration and liquefying of tissue. In case of resolution, the softening, liquefaction and absorption occur, leaving the part perfectly healthy.

As a last result of inflammation we may get gangrene,both moist and dry. It will not differ from that of active congestion, except that it will be more pronounced and will occur more frequently.

\section{DEATH.}

The time comes when every animal must die. With the veterinarian there is little sentiment about this event. It is impossible to define it; if we say that it is the cessation of life the question immediately arises, What is life? But we do not know. However, we consider animal to be a machine dependent upon three vital functionating systems:

1. Hydraulic system, - that including the heart and blood vessels.

2. Pneumatic system,- that made up of the respiratory apparatus.

3. Nervous system, - that composed of the brain, medulla, spinal cord and nerves.

In general, we may say that death occurs by interruption of one of these three systems every time. Interruption of the function of one will cause death, without reference to the other two. These three are the vital means of sustaining life, and if one ceases to functionate, the other two will also.

Some pathologists record a fourth channel through which death may occur,-the blood, the poisoning of which (necræmia) results in death. But this in turn acts upon the nervous system to which in such a condition the cause of death may be referred. 


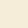



Death due to interruption of the heart's action is called syncope, and there are two kinds:

1. Sudden-sthenic.

2. Slow-asthenic.

Sthenic syncope (sudden heart failure) is purely functional. The nerves propelling the heart lose their irritability and the heart ceases to beat. In this condition there is no morbid anatomy, no premonitory symptoms of death. The heart stops beating suddenly:

In the asthenic form there is morbid anatomy and there are premonitory symptoms of imminent death. The horse will have repeated attacks of dangerous heart failure, indicating valvular insufficiency, or aneurism of the aorta, etc.

Sthenic syncope occurs in two forms:

1. Paralysis of the motor nerves of the heart, in which condition the heart is large, soft, and in a few hours after death will be full of a black postmortem clot.

2. Tonic spasm, in which condition the heart is small, hard and empty, i. e., when the animal is posted. The reason for this is that the heart contracted and failed to relax. If the animal had died during diastole, the heart would be large, soft and full; but during systole, death finds it small. hard and empty. This condition of the heart is seen in death from tetanus, in which there are tonic spasms of the whole body.

Sthenic syncope always occurs as the result of shock. This may happen during an operation, or as the result of a kick or some severe violence to the solar plexus. Shock is the condition caused by any violent disturbance of the nervous system.

Syncope may be caused by hemorrhage, and this is either external or internal. If the carotid artery, the jugular vein, or the femoral or brachial artery be severed, it takes no time for 
an animal to bleed to death. This is external hemorrhage; enough blood is lost to produce syncope. Internal hemorrhage occurs as the result of the rupture of some internal blood vessel into either the thorax or abdomen. Castrate a colt, say a bleeder, and as soon as the ecraseur is removed and if the cord is retracted into the abdominal cavity, the hemorrhage begins and in 5 or 10 hours the colt will bleed to death.

The symptoms of internal hemorrhage are:

1. Great and increased pallor-mucous membranes pale, finally white as paper. This is the most important diagnostic symptom.

2. Temperature of extremities varying according to the rapidity of the hemorrhage-cold if slow, and more or less warm if rapid.

3. Great and increasing weakness-as death approaches, in case of a hemorrhage requiring 5 or 6 hours to culminate, cold sweat breaks out on the surface. Then follows a dazed expression of countenance; the eyes get dim; the animal spreads his legs to prop his body; if down, he struggles convulsively to rise; the pulse becomes feeble, small and finally imperceptible; the expired air is cold; the breathing convulsive; and as death approaches the pupils dilate.

Death due to interruption of the pneumatic apparatus is called ASPHYXIA, whereby the animal is deprived of air.

The symptoms of asphyxia are as follows:

1. The mucous membranes are dark and grow darker. This darkening is called cyanosis. It does not occur suddenly, but comes on gradually.

2. Eyes bulge.

3. Nostrils dilate. 


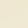

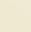

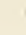



4. Breathing labored.

5. Cold sweat usually bedews the body.

In such a death the post mortem will show more or less cyanosis of all the organs, but especially of the lungs. There is general passive congestion. The blood turns black from the excess of carbon dioxide and from the decrease of oxygen. In the human, the face would be black, but a horse does not show cyanosis in his face.

Death by interruption of the brain and nervous system is called coma. A comatose condition is one of stupor, insensibility, and unconsciousness. When an animal dies from coma, the symptoms are as follows:

1. Prone on the ground.

2. Insensible.

3. Pulse slow-decreased in frequency way below normal.

4. Breathing slow and stertorous; slow, deep, irregular breathing and in some cases snoring, is always present in coma.

At the approach of death, the breathing keeps getting slower and more irregular, the pulse the same although it may continue to beat after respirations have stopped. In this condition of death the brain loses its irritability; the other organs are in normal condition.

When it does not pay to treat an animal, the veterinarian is expected to destroy it and this he must know how to do. The quickest and most painless method of killing is to shoot the animal through the fourth ventricle. An animal can lose nearly the whole of his cerebrum, large portions of his cerebellum without dying, but not the medulla.

\section{HOW TO KILL A HORSE.}

First, fill the gun with sufficient powder to drive the bullet through the cranium of the horse; a 22-calibre revolver will not do it. We use a 44-calibre gun, barrel about a foot long. Use long cartridges. 
Place one hand over the nose of the horse, holding his nose down toward his breast. Stand in front of him and shoot straight through the medulla. It is not necessary to place the muzzle of the gun against the forehead. If the animal is nervous, it may be necessary to blindfold him.

Do not attempt to shoot through the ear; the petrous portion of the temporal bone is the hardest bone in the body. But shoot high up toward the poll and you will hit the right spot every time. The horse shauld drop instantaneously. He dies from coma. The bullet causes shock; shock produces coma; and coma, paralysis of the brain.

The main objection to shooting a horse is the noise. There are other means of killing but they take longer and are more painful. Strychnine is the most active poison but it is very painful. Dissolve a large dose in water and inject into the jugular vein. When giving it by mouth, give at least 10 grains. Hydrocyanic acid is all right for killing dogs but it is too weak for the horse. The commercial acid does not keep its strength and the anhydrous is rare, we never get it. One-hundredth of a drop of the anhydrous acid on the tongue of a horse would kill him instantly.

Chloroform kills by paralyzing the brain and the animal dies from coma. Strychnine kills by syncope, tonic spasm of the heart. Hydrocyanic acid kills by paralyzing the heart. The chief objection to using chloroform is that the animal struggles severely during its administration. The feeling of suffocation caused by the chloroform produces violent struggling and in such cases the animal must be restrained the same as for an operation.

All poisons considered, probably strychnine is the best to use in killing an animal if you do not wish to shoot him.

Death through poisoning of the blood is called necræmia. The blood dies, or in other words is killed by some specific micro-organism, such as the bacillus anthracis. In this disease the blood is so disorganized by the germ that the corpuscles are wholly broken up and killed. This kind of death can be referred to the nervous system and we can say that the animal dies from coma, yet at the same time the dead blood is the cause of death. 

In order to determine the channel through which death occurs, one must note the effect of the disease processes. If the animal dies in a comatose condition, then the nervous system is the cause; if syncope sets in, then the heart is at fault. A person in a faint is comatose, but it is not deep enough to produce death; in real death from coma, the stertorous breathing must be present. All these conditions must be taken into consideration in order to determine the correct cause of death.

\section{NOSOLOGY.}

By nosology we mean the classification and nomenclature of disease. Diseases are divided into two general classes, constitutional and local. Of the constitutional there are two kinds, exogenous and endogenous; of the local, eight, these referring to the system affected; they are the diseases of the respiratory system, the digestive system, the circulatory system, the urinary, the nervous, etc. The following is a simple form of the classification of disease:

1. Constitutional-

a. Exogenous.

b. Endogenous.

2. Local.

a. Diseases of the Respiratory System.

b. Diseases of the Digestive System.

c. Diseases of the Circulatory System.

d. Diseases of the Urinary System.

e. Diseases of the Nervous System.

f. Diseases of the Generative System.

g. Diseases of the Locomotory System.

h. Diseases of the Cutaneous System.

A constitutional disease is one that affects the whole system primarily; if it localizes itself in any organ, it does so secondarily. A local disease is just the reverse; it is one that affects some part primarily, and the whole system secondarly, i. e., if it does so at all.

An exogenous disease is one arising from without the body, i. e., the cause is from without. This includes all of the con- 
tagious diseases, such as glanders, anthrax, tuberculosis, etc.

An endogenous disease arises within the body, the cause producing it is within the animal. This includes all non-contagious diseases, such as azoturia, acute constitutional lymphargitis, rheumatism, rickets, etc.

These constitutional diseases are sometimes spoken of as blood diseases. Local diseases include all the diseases not constitutional, and they are classified according to the part in which they are located.

\section{RATIONAL TREATMENT OF DISEASE IN GENERAL.}

Rational treatment is that prescribed according to the indications present. It is based upon the pathology of the case. Medicinal indications always should follow the pathological conditions. The prescriber should give attention to the individual case, and in order to do this, he must be a pathologist. He must first make a correct diagnosis and then follow the lesions up to the point where the case is presented to him. Rational treatment is based on the motto, Vis medicatrix naturæ, which means "the power of nature to heal." Our efforts must be directed toward the assistance of nature, help tide her over the rough places and difficult conditions.

Along the line of general treatment we divide diseases into acute, subacute, and chronic. Acute diseases require prompt and vigorous treatment what the object of either aborting the disease, or of lessening its severity and giving nature a chance to recover. The acute disease requires heroic treatment especially in its early stages, for it tends to run a rapid course, to develop and terminate quickly. For example, in intestinal flatulence, the horse is tympanitic, there is pressure upon the diaphragm causing interference with respiration, and a resulting cyanosis; the horse will die from asphyxia if he does not get immediate relief. The treatment must be prompt; if necessary let the gas off with a trocar and canula. This is heroic treatment, and many owners object on account of the possible danger, but it is the only way to save the life of the animal.

A subacute disease is mild, arises slowly, and is inclined to 
. 

terminate favorably. The rational treatment should be mild and need not be so prompt. Small doses less often repeated are given; in fact with no treatment at all, the case will recover.

A chronic case is one of long standing. The rational treatment is slow, and employs such remedies as tend to produce the absorption of inflammatory deposits.

\section{RATIONAL TREATMENT OF A FEVER.}

Ephemeral fever or fabricula is a fever in which it is the disease, there is no morbid anatomy. Fabricula is a simple fever.

Nature.-Fabricula is in itself the disease; it does not originate in any particular organ; runs a benign course; terminates favorably in 2 to 8 days. The nature of this disease considers whether it is febrile or inflammatory, the organ affected, the course the disease is likely to run, and its probable termination.

Etiology.-The main factors in causing a simple fever are exposure to wet and cold, fatigue from hard work, irregular feeding, improper diet, sudden change from the open air to confinement in the stable, change of climate, change from natural life to artificial,- any one of these conditions may cause a simple fever.

Symptomatology or Semeiology.-Fabricula comes on suddenly; a severe case will be preceded by rigor, the symptoms of which are:

1. Coldness of extremities.

2. Surface of the body cold.

3. Nose and ears cold.

4. Shivering-begins slight and increases in severity until the animal is shaking vigorously all over.

5. Breathing increases in frequency, and a peculiar whistling sound is produced.

The number of normal respirations in the horse is 10 , but in 
a chill, it may be increased to 60 per minute. The breathing is not labored, and there is no dilatation of the nostrils-these are negative conditions. As the chill grows worse, the horse hang; his head, droops his ears; this may last from 2 to 8 hours. The more severe the chill, the longer it will last, and the higher will be the fever following it.

To prescribe rationally, consider first the age and species of the animal; then the size, sex, vitality of the patient, duration of the disease, time of the year, the weather, and the surroundings. All of these conditions have their influence. The treatment consists 'of medicinal prescriptions and hygienic measures.

Suppose now you have a horse with a chill. The nervous system is excited, the blood has left the surface, the respirations are rapid, the pulse hard, rapid and strong. The sympathetic nerves control the heart and if we give Fld. Ext. of Aconite, this will reduce the irritability of the nerves. We prescribe with this a stimulant, alcohol, and the form of the prescription would be as follows:

\section{$\mathrm{R}$}

Fld. Ext. Aconite............... 1 drachm

Spts. Vini Rect................. 4 ounces

Aqua $q \mathrm{~s}$ ad.................... 1 pint

M. Sig. -2 ounces every 15 minutes until you get the physiological effect of the drugs.

The horse will sweat, and the heart will slow and the pulse get soft. Some will argue against combining a stimulant and sedative together, saying that the one neutralizes the action of the other, but experience teaches that this is not true.

Hygienic treatment consists in putting on woolen blankets and buffalo robes, bandaging the legs, shutting off the draughts, and rubbing the legs of the animal to increase the circulation.

As soon as the animal begins to sweat give the doses less frequently, and finally drop them off altogether. The dose above given, with proper hygienic measures will put the animal to sweating in about $2 \frac{1}{2}$ hours.

Suppose now that the disease has been running three days, you are called, say, the fourth day; you find the ears drooping, pulse 60 , temperature 105, feces slimy, etc. In this climate, usually some thoracic disease-acute bronchitis, pleurisy, or 


pneumonia may develop, but in this case let us say that it has not. Now what will you prescribe? A fever mixture, and this is our stock prescription.

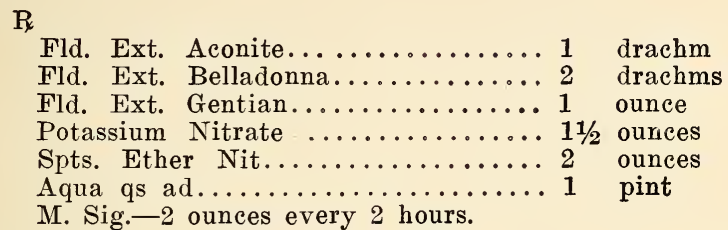

Make the dose small and repeat often, and in this way the physiological action will be produced gradually. Robertson in his Equine Practice gives large doses two or three times a day, but you will get better results with small doses every two hours. This impresses the owner so that he stays on hand and tends to his animal, and this produces better results.

As to the hygiene, water the animal every 15 minutes (you may be pretty sure that the owner has been afraid to give the animal anything to eat or drink) until he has had his thirst quenched, then put a bucket of fresh water before him, and keep it fresh. For fodder, give oats and bran mixed and scalded with boiling water. The boiling water ruptures the cellulose covering of the grain, and make it easier to digest.

The dose which I have given you is for an average weight animal, say 1,300 pounds. The aconite lessens the irritability of the nerves; the belladonna lessens the congestion; the gentian, a vegetable, helps to keep the stomach in order; the potassium nitrate is a diuretic and a refrigerant and helps to regulate the temperature. If ammonium chloride be added it will lessen the temperature more quickly. In addition to this prescription give a dram of acetanilid every four hours.

The bowels will take care of themselves, only take notice whether they move or not. If not, give a soap and water enema.

After getting the temperature down to 102, stop the acetanilid and put in fluid extract of nux vomica, 1 ounce to the pint. Drop the potassium nitrate. When the temperature gets to normal put the animal on Tr. Iron. Keep him quiet even if his legs are stocked. After the temperature has been normal for 48 hours, exercise may begin. 
The various procedures in making a diagnosis are as follows :

1. Inspection.

- 2. Palpation.

3. Palpitation.

4. Auscultation.

5. Percussion.

6. Succussion.

These are the means employed to make a physical diagnosis.

Inspection.-This implies a thorough observation of the animal before touching him at all. It includes the movements of the chest and abdominal muscles; the position of the elbows; the expression of the countenance, which is quick to indicate distress; the extent of dilatation of the nostrils; the amount and character of any nasal discharge, and the appearance of the coat.

Mensuration.-This implies the measuring of any part and the comparison of it with the opposite side. The measurements are usually sized up with the eye rather than with a tapeline; in this way the eye becomes trained to routine practice.

Prof. Gamgee was a great stickler for tapeline measurements, but he was more scientilic than practical.

Palpation.-This is the act of feeling. See if the surface is cold or hot. Manipulate the parts to find if any soreness be present; feel of the coat; count the pulse; take the temperature; when indicated, lay the hand upon the horse's hip and feel the impulse of the labored breathing, for it is an important point in diagnosis.

Auscultation.-This is an application of the ear to the wall of the body cavities to ascertain whether the sound be normal or not. The ear can be applied directly to the surface or a stethoscope can be used. In veterinary practice the ear is more commonly used.

Percussion.-This is the tapping of the surface over cavities to ascertain the density of the cavity. The practitioner must be familiar with the normal resonance of the body cavities. This varies according to the amount of flesh overl:eing the cavity. A thin horse has greater resonance of his body cavities than a fat one. Dullness of the chest indicates disease; dullness of the 


facial sinuses indicates that they are filled with mucous discharges or pus; dullness of the abdominal cavity is normal, and it becomes resonant only when filled with gas.

Succussion.-This is the act of shaking a patient so as to detect the pressure of a liquid in the cavities of the body.

\section{RESPIRATORY DISEASES.}

The phenomena of the diseases of any particular system are the various symptoms and signs which call one's attention to it.

As applied to the horse, the various phenomena of the diseases of the respiratory system are as follows:

1. Grunting. In cattle grunting occurs as

a symptom of digestive diseases; in

the horse never as such.

2. Coughing.

3. Dyspnœa.

4. Roaring.

5. Whistling.

6. Rales-called mucous rales.

7. Crepitation.

8. Friction sounds.

9. Sneezing.

10. Snoring.

11. Wheezing.

Grunting.-The grunt is present in acute pleurisy, and indicates painful respiration.

Coughing.-A cough is a forcible and sudden expulsion of air from the chest. It begins with a sudden and deep inspiration, and then all the respiratory forces come into play to expel the air,- the diaphragm, the intercostals and the abdominal muscles. A cough may be either moist or dry. The moist cough is heard in the second stages of acute respiratory diseases; the dry, in the first stage of the acute and in the chronic respiratory diseases. A cough may be shallow or deep; the shallow is from the throat, and the deep from the bronchi. The throat cough 
is a hacking, short, sharp and shallow one; the bronchial cough is hoarse and deep.

Dyspnoea.-This is labored breathing, interrupted more or less by some interference. It may be slight or severe, and it may be fatal. It is seen in either acute or chronic diseases. In the acute disease it requires prompt attention, but in the chronic it is not dangerous.

Roaring.-This is a recognized name of a disease, and is due to the dropping into the larynx cavity of the left arytenoid cartilage.

Whistling.-This is a shriller noise than that of roaring. It may be produced by anything that constricts the caliber of the air-passage.

Rales.-This sound is produced by air passing through a liquid, such as mucous, pus, blood, water, medicine, etc.

Crepitation.-A crackling sound heard in the early stages of pneumonia.

Friction Sounds.-This is the sound of two dry surfaces rub-. bing together, as heard in the second stages of acute pleurisy.

Sneezing.-Among the lower animals, this is simply blowing the nose, there is no cough.

Snoring.-A noisy breathing produced usually by a polypus in the posterior nares. It is occasionally the result of nervous disturbance, seen in nervous high-bred Jersey cows. Pug dogs always snore.

Wheezing.-This is the sound heard when the air passes through a more or less obstructed passage, such as would be caused by a nasal polypus, enchondroma of the septum nasi, or the asthmatic exacerbation of heaves.

\section{RHINITIS.}

Definition.-Rhinitis is the inflammation of the mucous membrane lining the nose. It is also called catarrh, which is derived from the Greek katarrein, meaning to flow down, and always refers to the discharge of a mucous membrane. The name catarrh can be applied to any mucous membrane of the body, but it is usually applicable to the nasal membrane. 


\section{.}



Nature.-Rhinitis may be acute, subacute, or chronic. In the acute form inflammation of the Schneiderian membrane is present; it extends back and involves the mucous lining of the fauces. In a very severe case, it involves the sinuses of the head, the frontal and the superior and inferior maxillary.

Etiology.-This inflammation is usually brought on by exposure to cold winds, or to sudden change of temperature. It can occur in hot weather as well as in cold. In Chicago the northeast wind is apt to cause catarrh in horses that are exposed to it after exercise.

Symptoms.-The first symptom is usually sneezing; pain is probably present in the Schneiderian membrane. After a few hours a slight cough develops; elevation of temperature-103 to 104 ; pulse 48 to 50 ; lassitude; congestion of the mucous membrane of the nose and eyelids.

Pathology.-The inflammation runs three stages; first, the mucous membranes are dry and their function is suspended, second, this is the moist stage and a red, limpid, thin, water discharge begins which in 12-20 hours becomes starchy; third, if the case is bad, suppuration develops, and the discharge is mucopurulent-pus mixed with mucous. In the first stage the function of the mucous membranes is suspended, but as soon as reaction begins, they begin to act again and pour out their discharge, which, however, is not normal, but is degenerated,-a thick, starchy coagulated substance. Not all the mucous follicles suppurate, and what normal mucous is poured out becomes mixed with the pus and degenerated coagulum. In time the discharge may collect in the sinuses, the swollen mucous membranes preventing its escape. It then becomes very offensive, because of decomposition of the mucous element. The ordinary discharge from the nose is usually odorless.

Everitually the mucous membranes may ulcerate; the periosteum of the bones desquamate.

Treatment.-The acute form of rhinitis is a febrile disease and therefore needs antiphlogistic treatment. The following procedures are recommended:

1. Put animal in a comfortable place.

2. Let him rest; do not work him. 
3. Dose him with acetanilid.

4. Give a fever mixture, such as recommended in ephemeral fever.

5. Give a diuretic-the kidneys must be stimulated early in every febrile disease, so as to prevent an accumulation of debris in the urinary system.

6. Apply local treatment to the head.

Steaming the head over a tub of hot water is good for this case. Set a wash or soap tub on top of a barrel, and tie the horse's head so that he cannot thrust it down into the hot water. Throw a blanket over his head, and a red hot brick into the tub of water; this will precipitate suddenly a lot of steam, which will bring relief to the animal if the inflammation is located in the head, but not if in the lungs. It is possible to medicate the steam with an ounce of white wine vinegar, or dilute acetic acid, both of which are soothing to inflamed mucous membranes. If the catarrh has reached the suppurative stage, throw an ounce of carbolic acid or an ounce of tincture of iodine into three gallons of water (hot), and allow the steam of it to penetrate the animal's head. These will. act as antiseptics. (Empirics will burn old leather and old boots, thinking that the fumes will be soothing to the inflamed membranes of the head, but this is erroneous.)

If the sinuses of the head are involved, the case is severe; but if not nature will effect her own cure. The nature of the discharge will enable one to tell whether the sinuses are affected or not. If they are, after steaming, rub a little ammonical liniment on the overlaying skin.

$\mathbf{R}$

Aqua Ammonia ............... 1/2 ounce

Ol. Terébinth ................ 1/2 ounce

Ol. Gossypii................ qs ad 2 ounces

M. Sig.-Rub a little of the liniment over the sinuses-frontal and maxillary-night and morning; in severe cases apply to the throat and fauces.

(Linseed oil can be used instead of the cottonseed.)

After following this treatment for two or three days, the case is practically cured, but you must dry up the discharge and put the animal on a chalybeate course,- - a course of iron. 


R

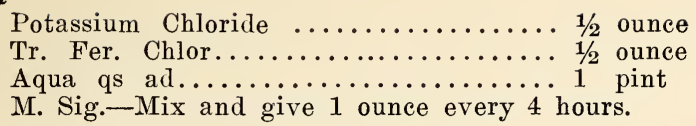

In a bad case keep the horse in for a week. As soon as the acute symptoms have subsided discontinue the steaming; stop the use of the ammoniacal liniment as soon as the skin begins to wrinkle, and apply a little cottonseed oil to soften up the skin.

Subacute Rhinitis.-These cases are mild and will not require much if any treatment. Probably the case will not be brought to you in this stage. However, acute cases may develop into chronic and affect the sinuses of the head.

Chronic Rhinitis or Catarrh.-This is sometimes called Ozena. It is a chronic nasal catarrh. Among the English Veterinarians it is known as nasal gleet. Chronic catarrh always follows the acute if it occurs at all. It has constantly to be differentiated from glanders. Its symptoms are as follows:

1. An intermittent mucco-purulent discharge, of heavy specific gravity, and nearly always offensive. The color varies from greenish yellow to whitish yellow, according to the amount of coagulated mucous in it.

2. Chunky condition of the discharge following the intermittency. This is due to coagulation taking place during the confinement of the discharge over night, and it becomes very offensive. As soon as the animal begins to exercise, chunks of coagulated pus and mucous, fetid and very profuse, pour out of the nose until the sinuses are empty.

3. Cough may be present; usually the submaxillary lymphatics are enlarged.

4. Bulging of the sinuses from the pressure of the muco-purulent material within. In such a case the eyes may water a 
little, the conjunctiva be red, and the corners of the eyes contain a little coagulated mucous.

5. Appetite usually good.

6. Coat looks well if the animal has been kept in good condition.

Negative symptoms :

7. No ulceration of the Schneiderian membrane.

8. If the mallein test is tried, it proves negative.

Treatment.-The treatment of chronic catarrh is entirely dependent upon the condition of the animal when first seen. If the disease has been running for the previous three or four weeks without treatment, or even if treated before, try to cure the case without any operation. Do this by using a nasal douche.

Tie the horse from each side and fasten him down, holding his face as nearly perpendicular as possible. Twitch one ear and insert the douche in the nostril. Do not place the twitch on the nose in this case. Insert the douche in the side running the most, using a mild lotion. A good lotion can be made from a 10 per cent solution of sodium chloride; sodium bicarbonate: boracic acid; or borax. Borax is borate of sodium. If carbolic acid is used, make up a solution about one-half of one per cent strength. Do not use solutions stronger than those mentioned. Rinse the nose with a solution of permanganate of potash1 to 1000 -then use the salines. Run through the douche about 2 or 3 quarts or a gallon of this. Internally give the horse iron -exsiccated sulphate-one dram in the feed night and morning. In addition to this, if you wish, give him Fowler's solution of arsenic, one-half ounce night and morning. This dosage would be for a 1000-1b. horse; for a 1600-1b. animal, give one ounce night and morning. Keep all this treatment up for two or three weeks, and feed on soft feed, grass if possible. If the sinuses are not too severely affected, this will cure the horse; but if they are full of mucous and pus, of degenerated and decomposing material, or if the sinuses are bulging, then an operation is necessary. 

Operation.-Put the horse on the table or lay him down, using a soft cushion under his head with a strong man to help hold him. Cut out a circular piece of skin opposite the fourth molar tooth, making the opening large enough to insert the instrument. Cut away the cellular tissues so as to expose the bone. Then bore through and withdraw the instrument and cut out the mucous membrane. In a long-standing case the pus will be very thick, so thick that it may have to be spooned out. Do not try to get all the pus out. Syringe the sinus well; the air and water will soften the mass so that in 60 to 72 hours the pus will come out easily. After the operation syringe out the cavity with a mild solution such as previously mentioned, and repeat night and morning. Hang an apron over the horse's head to cover the opening, and leave the hole open. The air will do as much to effect good healing as anything that could be applied. Some practitioners use a cob to stop up the opening, and corks are also used, but the wound should be left open. Nine times out of ten a case will yield to this treatment. After about three weeks, it will not be necessary to use the syringe longer, as the mass will have become so soft that it can easily be removed. The first two weeks, syringe out once every day; thence once every three days. Too much washing maintains a discharge. Nature will do better than local applications.

If you meet with a case that has been running a year or longer, the treatment may fail. There are many cases in which the mucous membrane is entirely gone as the result of absorption. Keep the horse on iron all through the treatment; it helps materially to arrest suppuration.

This operation is simple and usually satisfactory. Bear in mind that the fetid breath is a symptom of chronic nasal discharge. When this condition is present, always examine the teeth to see that they are not decayed. If you find a decayed molar, remove it first; but if the mouth is sound, get after the nasal catarrh. It is very easy to mistake this condition for glanders.

In removing a tooth, two things are to be guarded against, the cutting of the blood vessels and also of the nerves that supply the roots of the molars. Work above these and do not dis- 
turb them if possible. Although a very painful operation it is not advisable to use chloroform.

\section{LARYNGITIS.}

Definition.-Laryngitis is the inflammation of the mucous lining of the larynx. Theoretically it may be limited to the larynx, but in every day practice, we usually find that it involves the pharynx. It is then known as laryngo-pharyngitis. This is the usual case we find to treat, and it is spoken of as sore throat. In bad cases three or four inches at least of the upper part of the trachea are involved.

Pathology.-In this disease the inflammation runs the regular typical course; first the dry, hot stage with suspension of the function of the mucous membrane; then the second or the wet stage with an abundant effusion producing more or less œdema. This œdema makes the parts swell; if it is located mostly around the rima glottidis, it will diminish the size of the air passage and cause dyspnœa. Sometimes the passage becomes closed entirely, and the animal dies from asphyxiation. This œdematous condition merits a special name-oedema glottidis. It may result in asphyxiation in 48 hours. This is always a dangerous condition in man or beast, but more particularly in children under 5 and in people over 60 . The first stage is short, 5 or 6 hours only. Sometimes the inflammation goes on to the third stage,puration, but this never amounts to much. Occasionally the case is so severe as to produce local necrosis in the larynx, and the horse in coughing will throw out shreads of necrotic mucous membrane. This condition is called necrotic laryngitis, and the part coughed up is called a "sphacelus." The act is called "sphacelation." This is usually fatal, and in 6 or 8 weeks the horse dies from septicemia. In the horse this is a very dangerous condition because the larynx cannot be reached to clean it out.

Death from œdema glottidis usually occurs within 48 hours if it occurs at all; after that there is less danger.

Etiology.-The cause is undue exposure. Take a barn holding 40 horses-brick barn worse than frame-wide open, no fire, 

and very cold. The bricks hold the chill. The horses feed out at noon in nose bags, and come in at night tired, very much so if the ground is covered with snow and ice. They cannot resist the chill of the barn, and the next morning one horse does not eat his breakfast. He has acute laryngitis.

Symptoms. - In all bad cases there is more or less chill, which the attendant may or may not see; temperature 104-106; pulse full, round and soft, and increased in frequency; throat swollen, outside and in (the more it swells on the outside, the less it swells on the inside), and those swelling freely on the outside are not the most serious cases; nose poked out. If this were seen in the first stage, there would probably be a dry, sore, hacking cough with disinclination to swallow. In the second stage an œdematous, moist cough is present, and it is painful. The pain is indicated by the horse shaking his head after each cough. The discharge is profuse, mostly saliva. the reason for which is that the inflammation is in close proximity to the parotid glands, and stimulates them to increased functional activity. This secretion passes back through the fauces but cannot go further down on account of the swelling, and is therefore forced out through the nose. In this condition the throat is sore; pressure on the outside causes the animal to throw up his head, an indication of pain.

The case runs along for 4 or 5 days, by which time there may be a little pus, a few mucous follicles may suppurate, and the discharge may be starchy. However, is usually consists of mucous and saliva. The breathing is accelerated; upon opening the mouth it is full of ropy saliva. In all these cases there is more or less dyspnœa, and the mucous membranes become cyanotic according to the amount of dyspnœa present. The less oxygen the horse gets the darker the mucous membranes become. If the dyspnœa becomes severe, the eyes bulge, and the animal makes a great noise both during expiration and inspiration. Death may come any time between 48 and 100 hours, usually in about 75 hours. As death comes on the extremities get cold, the eyes bulge, and weakness develops rapidly; but the animal persistently stands until he drops dead.

Prognosis.-This depends entirely upon the amount of dysp- 
nœa present. Laryngo-pharyngitis with œdema glottidis is very dangerous and often fatal. If it takes three or four days for the asphyxia to kill him, the mucous membranes will not only be cyanotic, but they will become ecchymotic as well.

Treatment.-As a rule the doctor is called the first day; he sees the horse not eating and discharging from the nose. The following is the course of treatment to pursue:

1. First look after the hygiene, warm up the stable-with a stove if possible. Keep the temperature 60-66 degrees. You cannot treat an animal successfully in a cold barn.

2. Clothe warmly.

3. Apply the ammoniacal liniment on the outside of the throat freely. The old theory of a blister supposed its action to be that of a revulsive to draw the inflammation out and to drive it away; but this theory is obsolete. We know now that it acts as a local stimulant, setting up a mild inflammatory process; and in an acute disease this is always beneficial.

4. After applying the liniment, then put on a hot linseed poultice, which must be repeated night and morning. Do not repeat the application of the liniment. Bandage over the poultice well.

5. For internal medication give a dram of acetanilid and repeat every 4 hours.

6. Give an ounce of fever mixture every hour-small doses will insure that more be swallowed.

7. See the case every day. If the inflammation increases, give a camphor electuary. 

$\mathbf{R}$

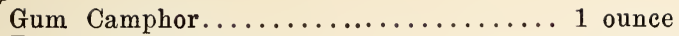

Fl. Ex. Belladonna...............2 ounces

Fl. Ex. Glycyrrhiza...............2 ounces

Fl. Ex. Nux Vomica............. 1 ounce

Theriacae ...................6.6 ounces

Pharmacy.-Powder the gum camphor in a mortar, and add a little alcohol. Then add the fluid extracts and stir up and then the theriacæ. Stir all up together, adding enough powdered glycyrrhiza, probably 2-4 ounces, to make the mixture thick enough to administer the dose on a wooden spatula. In administering a dose, simply take it up on the spatula and wipe it off on the tongue of the animal. The advantage of the method is that the dose stays in the throat longer.

If the case does not yield to these measures of treatment, do not wait too long for an operation; as soon as the mucous membranes become cyanotic, tracheotomy is indicated. There are several different kinds of tracheotomy tubes and they vary in size to suit the animal.

Tracheotomy.-Make an incision 2 inches long through the skin to the cartilaginous rings, then turn the scalpel crosswise and cut through the membrane of the trachea. Enlarge the opening with a probe-pointed bistoury. Do not wound the cartilage rings. Insert the tube and fasten around the neck well. The tissues overlaying the wound and adjacent to it may begin to swell and force the instrument out, but this need not occur if the operation is aseptic. The tube should be removed and cleaned every night and morning.

If this operation does not cure, keep up the other treatment until the disease begins to yield.

An ordinary sore throat is cured by the iron gargle:

$\mathbf{R}$

Tr. Fer. Chloride................ 1 ounce

Potassium Chlorate ................ 1/2 ounce

Aqua qs ad...................... 1 pint

M. Sig.-One ounce every four hours.

In cases not severe enough for the camphor electuary, alternate the gargle with the fever mixture. Also use the gargle during convalescence.

Hygiene.-Keep fresh water before the animal all the time; give him anything he wants to eat. 


\section{CHRONIC LARYNGITIS.}

Under this head come nearly all the conditions producing noisy breathing-roaring, whistling, wheezing, thick wind, chronic cough, and paralysis of the throat.

Etiology.-These chronic conditions generally follow some acute disease of the throat, but they may not. Roaring, for example, is due to paralysis of a nerve; whistling may be caused by a certain anatomical conformation; wheezing, diseases of the nostrils; thick wind may follow bronchial diseases; chronic cough-anything; paralysis of the throat may follow acute laryngo-pharyngitis.

Roaring.-Roaring is due to the paralysis of the left recurrent laryngeal nerve. As a result we get paralysis of certain arytenoid muscles. These are the arytenoideus, thyro-arytenoideus, crico-arytenoideus, and the crico-arytenoideus posticus. In this condition the muscles atrophy and change their red, fleshy color to that of pale yellow or amber. As a result the arytenoid cartilage drops into the larynx and obstructs inspiration. Expiration is performed with these, but in inspiration, the rima glottis may be almost closed by the obstructing cartilage.

Symptoms.-These consist of loud roaring sounds in breathing, great difficulty in respiration,--may be so great as to choke the horse until he drops. But the animal does not die from asphyxia; when he goes down, the recumbent position helps restore respiration. So long as the horse is quiet, there is no difficulty in breathing, but the roaring occurs during exercise. As a rule, roaring ruins the horse, although the famous Eclipse was a roarer.

Whistling.-This is a sharp shrill sound on a high key. It is sometimes produced by diseases of the throat. It can be produced mechanically. A horse with narrow jowls harnessed with a curb bit, under the tap of the whip will produce pressure on the larynx so that it becomes constricted, and this may cause whistling.

Wheezing.-This disease is not necessarily connected with the larynx, it may be in the nose. It may be caused by neoplasm of the septum nasi, polypus in the nostrils, fracture with 


depression of the nasal bone, and general swelling of the mucous membranes as seen in glanders. The cause may be in the chest in the form of bronchitis, for example, in cattle; in bad cases of heaves in which there are asthmatic exacerbations.

An abcess in the wall of the throat is the usual cause of wheezing, i. e., when its cause can be referred to the throat. Tympanitis of the guttural pouch may cause it-a congenital malformation. Wheezing may also be caused by fracture of the tracheal rings with depression. Stenosis of the trachea following tracheotomy may cause it.

Treatment.-The treatment is usually surgical. Some cases may be treated successfully with an external application of a blister while iodide of potash is given internally.

Chronic Cough.-This condition is generally incurable. It may come as an accompaniment of any of the chronic laryngeal troubles. It may come from heaves. It is a short, hacking throat cough, usually in spasms. The horse coughs once, or a half dozen times, then stops. In roaring and other chronic laryngeal diseases, there is usually a chronic cough and it may be the only thing the matter. Pinch the iarynx to locate the place of irritation.

Treatment.-Increased functional activity is desired, therefore apply a fly blister over the larynx. The blister should be mild; too severe an application will blemish the thin skin which covers the throat. After removing the blister grease the throat and wash it. Repeat the blister from three to six times, six weeks apart. Internally give iodide of potash (1 dram for a 1,200 $\mathrm{lb}$. horse) two or three times a day for a month, or twice a. day for three months. Some cases will yield to the iron gargle. 1 ounce every four hours. Guaiacol can be given also.

$\mathrm{R}_{\mathrm{k}}$

Guaiacol $\ldots \ldots \ldots \ldots \ldots \ldots \ldots \ldots \ldots, 1$ ounce

Simple Syrup or Linseed Oil......... 15 ounces

M. Sig.-Give 1 ounce 3 times a day.

A valuable addition to this is a little heroin, $11 / 2$ grains to the ounce, especially if the cough is painful. Or a little codine can be given. These quiet the laryngeal nerves.

In an ordinary case of strangles after the abscess has been 
treated, the guaiacol mixture with a fly blister on the outside, is good treatment.

Hygiene.-Keep the horse on a grass diet or at any rate on soft feed.

Paralysis of the Throat Following Laryngo-Pharyngitis.This is a very peculiar condition; it is a wasting of the soft tissues of the throat. The skin is drawn tightly over the atlas, and the outline of the hyoid bone can be seen. The neck looks like a skeleton covered with skin. There is paralysis of the muscles of deglutition, difficult swallowing, persistent coughing and the food and water come back through the nose. The horse runs down in flesh, is weak, and the case becomes chronic. This disease is liable to occur any time, but it does not occur often.

Prognosis.-The prognosis is usually favorable if thorough treatment is carried out.

Treatment.-Apply a fly blister to the outside of the throat, and repeat 5-6 times a month apart, being very careful not to blemish the skin. Put the animal on iodide of potash and strychnine. Give a grain of strychnine with 1 dram of iodide of potash in two ounces of water 3 times a day for 2-6 months. The cases all recover.

\section{QUINSY.}

In the human, quinsy is inflammation of the tonsils in which abscesses form. But horses have no tonsils, and consequently they do not have typical quinsy. Pigs, however, can have quinsy, for they have tonsils. But in the horse, abscesses gather occasionally in the lymph glands about the throat as a result of laryngo-pharyngitis. We get quinsy also in some cases of strangles. Pus may gather in the guttural pouches-in one or in both-and becoming confined there, produce pressure and dyspnœa.

Etiology.-Quinsy is always secondary to severe inflammation of the throat. When it comes with strangles, it is easy enough to account for it, but in the other cases it is not always understood. These abscesses, however, probably always follow some iocal inflammation. 

Symptoms.-The symptoms consist of swelling on one or both sides; if on both, dyspnoea; swelling hot, hard and sore under pressure; usually a rise of temperature; pulse 50 ; mouth full of ropy saliva; mucous membranes discolored according to the amount of dyspnoea present. The abscess may form posterosuperiorly to the pharynx (post-pharyngeal abscess) in the centre, but usually develops on one side more than on the other. In all these cases the nose is poked out. Pressing the nose toward the breast causes great pain; if done suddenly, may make the animal rear.

Treatment.-When the abscess present itself by an external swelling, surgical interference is indicated. Being deep-seated, these abscesses will not point and rupture spontaneously, although they may occasionally do so, and 9 times out of 10 they have to be opened. When called to such a case, find out the history, the length of time the case has been developing, etc., and then operate 5 days from the time the disease began. If the case has been running only 2 or 3 days, order a hot poultice. Change night and morning until the abscess is 5 days old, when the pus will have gathered sufficiently to be easily found, and then the abscess can be evacuated.

Operation.-The seat of operation is a dangerous one, there are so many large blood vessels to be avoided. If the abscess is superficial, there is not so much danger. Above the larynx and in front of it there is a small triangular space in which to cut,- - the external carotid and jugular, must be guarded against. Clip the hair over a place 2 inches in diameter, and make a puncture through the most prominent point of the swelling (put intermittent pressure on the jugular below so as to easily see the vein) and incise the skin with an inward, up and outward motion of the knife. Then turn the knife down and push it into the abscess slowly until resistance ceases. Then you are into the pus sac, and when you withdraw the knife, the pus will come out. With a probe pointed bistoury cut up or down as conditions will permit, being careful not to cut off the blood vesse's, and enlarge the opening sufficiently to admit the finger. Slow cutting causes less shock than fast. Stabbing will produce shock.

Syringe out the abscess and inject 1 pint of antiseptic solu- 
tion (bichloride 1-500). first and then use a bichloride solution 1-1000 until the abscess is clean. Soak a pledget of oakum in the bichloride solution and insert into the cavity, allowing it to protrude a little, and then put a hot linseed poultice over the place, mixing with the meal a little of a 5 per cent solution of carbolic acid. This poultice will reduce the inflammation quickly. Dress night and morning until the discharge has ceased, then stop the packing, smear the surface with vaseline, and put on a flannel hood to keep the parts warm. In about a week the horse can go to work.

Occasionally you may have a hemorrhage. Do not stop operating, but go on quickly and finish; then pack the cavity fuller than you otherwise would, and take a stitch or two in the skin to keep the oakum in. Do not dress for 24 hours and the cut vessel will have ceased to bleed.

Post-pharyngeal abscesses are treated in the same way.

\section{DIPHTHERIA.}

In the human, diphtheria is a specific inflammation of the throat with the formation of a false membrane. It is a constitutional disease of the exogenous type. The diagnosis is made by making a microscopical examination of the scrapings from the throat, in which will be found the bacillus of diphtheria.

Treatment.-This consists in the administering of anti-diphtheritic serum. The serum is obtained from horses that have been inoculated with cultures of the bacillus until no febrile reaction follows. The neck is washed and shaved, and the jugular is tapped, and the blood is drawn off into a sterile vessel. After the blood has clotted, the serum is removed and treated in some way to prevent decomposition, and then is put up in sealed cases for distribution.

The horse is used for this work because he is especially healthy and his tissues are good culture media for the germs. $\mathrm{He}$ is only used once for this purpose and is then sold.

There is no disease among the lower animals corresponding to diphtheria in the human, 




\section{BRONCHITIS.}

Definition.-Bronchitis is the inflammation of the mucous membrane lining the bronchi or bronchioles. It is divided into three classes, acute, subacute and chronic. The acute form affects the large bronchi mainly, but in severely acute cases the bronchioles may become involved. The inflammation is catarrhal in nature on account of the free flow of mucous present. Occasionally the air vesicles are also involved in the inflammation, and pneumonia is the result. We may have bronchitis and pneumonia running at the same-broncho-pneumonia.

Bronchitis is very common in the horse, dog and man. The same phenomena are in all cases. It usually runs the first and second stages of inflammation and then an absorption of the inflammatory products takes place, the case terminating by resolution. But it may become chronic, especially in man and cattle.

Acute.-The inflammation runs from 10 days to 4 weeks and terminates favorably. In an unfavorable case, say one which runs on to the third stage, the mucous follicles suppurate and an absorption of the toxins and poisonous products takes place and $90 \%$ of such cases die from septicemia in 2-4 weeks. Another fatal termination of the case occurs in the second stage. In this case the effusion is excessive and occludes the bronchioles. The animal would die quickly from asphyxiation if the condition were extensive enough. But it is usually localized and the air in the vesicles of the occluded bronchioles becomes absorbed and the vesicles collapse, producing atelectasis, the so-called foetal lung. It is small, blue, more or less solid and does not crepitate.

Etiology.-The cause of bronchitis is usually exposure to cold when the animal is tired. Exhaustion lessens the powers of resistance. Acute bronchitis may develop from laryngitis. It may be produced by impure air, such as we find in close stables, on board ship, etc. Introduction of foreign bodies into the bronchi, medicine, food or any other body, will cause bronchitis. It is more common in children and dogs. Attempt to drench an ox which is in a comatose condition and the failure of the epiglottis to close down may cause asphyxia and at any rate acute 
bronchitis. Gastric flatulence in the horse is often followed by acute bronchitis because of the irritation produced by vomited matter falling into the air passage as it passes over the larynx and descend to the bronchi. Gastric flatulence produces great nervous prostration and the glottis, its nerves and muscles, are depressed. In drenching a horse do not elevate his head too high. It is bad practice to drench a horse through the nose. Inhalations of smoke and hot air in burning stables may cause bronchitis.

Symptoms.-Bronchitis is usually ushered in with a rigor if the inflammation, follows exposure. The mucous membranes are injected. The first stage runs a rapid course and the cough is deep, hoarse and dry. The temperature is 105-107 and the pulse 50, soft, full, round and regular. The respirations are 18-20 per minute. The stage lasts about 6 hours.

In the second stage effusion begins with a limpid reddish discharge from the nose. The expired air is hot and by auscultation over the front of the neck at the base of the trachea a loud rasping sound can be heard. In a few days the mucous membranes secrete an abnormal mucous that coagulates and looks like starch. In the bronchi some effusion occurs, and there is a lot of mucous that does not coagulate. Mucous rales can be heard at the base of the neck. The nostrils are dilated, the appetite usually lost and secretions altered, and the excretions scanty; cough moist and suppressed, painful and made with the mouth closed.

Course.-In the first stage we find the head hung low; ears drooping; visible mucous membranes red; pulse 55 ; respiration 20 ; temperature 106-108 according to the condition of the horse.

In the second stage the first evidence will be a watery discharge from the nose, while the pulse becomes rapid and softer. Respiration by this time probably will get up to 24 and pulse probably 60. Expiring air hot and loaded with moisture, especially plain if the horse is in a cold atmosphere. By this time the appetite is less and the thirst probably a little increased, feces inclined to be slimy, discharge from the nose quite profuse and more or less starch like, cough deep, painful and suppressed. When the horse coughs he arches his back, lowers his head and 


\section{.}



refrains from coughing as much as possible because it is painful; coughs with his mouth shut, which is about the only case where this occurs.

This stage is very indefinite in length, may be from 3-20 days. In moderately bad cases the mucous rattle will be heard in the large bronchi. In a very severe case you will hear a squeaky sound in the outer third of the lung. When you hear this you can conclude that you have a very serious case on hand. These sounds are spoken of as "sibilant" sounds and indicate a very severe and dangerous case. They indicate that the bronchioles are involved and the condition is so distinct that it has been given a separate name called Bronchiolitis, which is an inflammation of the mucous lining of the bronchioles. In the first stage you are not likely to hear a rattle in the outer third of the lungs but as the case runs into the second stage and through it, the effusion is more plastic than that in the large bronchi, so much so that another name is given to it; it is called "fibrinous," which indicates a coagulable character and suggests possible dangers-effusion and exudation and tendency to coagulate and plug the bronchioles. In this case under auscultation there will be more or less dullness or possibly more or less absence of sound, which will compel you to consider the case very dangerous. This may involve any part of one lung or both lungs. If both lungs are involved the horse will surely die. This dullness or partial absence of sound under auscultation indicates occlusion of the bronchi. In a favorable case the fever subsides and the mucous rattle gradually grows less, coughing is less often and painful and the appetite returns. The horse has been persistently standing up to this time, but he may soon lie down and the lungs recover by resolution. In neglected or prolonged cases the horse may retain his cough for many months or even life.

In the third stage (suppuration) some of the mucous glands suppurate, the discharge from the nose then becomes mucopurulent. The odor of the expiring air is fetid. This fetor is a positive indication of the third stage. The temperature usually remains around 103 to 104 , possibly dropping a little; pulse 60 , 70,75 or 78 , weak, soft and small, appetite poor; horse loses 
flesh rapidly and dies in from 2 to 4 weeks from septicemia. The fetor of this expiring air is so marked that you can smell it plainly.

$90 \%$ of the cases of bronchitis in the third stage die and it is safe to say that at least $90 \%$ of the cases that do not run beyond the second stage recover. In cattle acute bronchitis is more apt to prove fatal than in horses.

Special Pathology.-In the first stage the ciliary epithelium is ruffled up producing the sensation of rawness that we feel in such cases and the mucous membrane is intensely congested.

In the second stage the effusion of serum increases and there is a secretion of degenerated mucous that coagulates more or less. This serum macerates the attachments of the ciliary epithelium and they become loose and float out with the discharge leaving the surface unprotected. If the disease is confined to the bronchi no further development will take place unless it goes on to the third stage, which is that of suppuration. In that case some suppuration will take place and probably ulceration. This ulceration in all probability leads to a fatal termination by absorption of the putrefactive matter. In cases of bronchitis the effusion is drawn back by inspiration, leading to the plugging of the bronchioles. This condition is spoken of as "occlusion." In some cases some of the thinner portion of the effusion or mucous may be forced back into the vesicles. The air in the vesicles at the ends of the occluded bronchioles becomes absorbed. In that case the vesicles collapse, which constitutes atelectasis, producing what is known as "fetal lung."

Post Mortem.-There are three stages in bronchitis in which horses may die. One is when the effusion in the bronchi is excessive, filling the smaller bronchi and the horse dies from asphyxiation, practically in the same condition as an animal would be under water. In bronchitis with effusion the medium sized bronchi are full and the horse practically drowns. The second is when he goes into the third stage and dies from septicaemia. Third, when he has bronchiolitis with occlusion and atelectasis.

The post mortem characteristics are as follows: The large bronchi are about half filled, we will say, with a frothy, bloody, 

sero-mucous liquid; the smaller or medium sized bronchi will be found full of this. Cut the lung across and upon pressure large quantities of this liquid will be forced out of the bronchi. If the horse dies in the third stage pus will be present. If you lay open the bronchi you will find more or less ulceration. The affected part of the lungs will be blue, noncrepitant, usually with small whitish spots or specks, about a quarter the size of a pinhead under the pleura. These specks are mucous or serum which is forced back into the vessels in the effort to breathe.

Treatment of an average case with a temperature of 107 following a chill; horse weighing $1200 \mathrm{lbs}$; pulse 55 ; respiration 20 ; visible mucous membranes red, head hanging, ears drooping.

Criticise the stable; see that the horse does not stand in a draft and gets plenty of fresh air. Ventilate the stable well. Put in a box stall if possible. Put a stove in the barn under the window, a thermometer near the horse and keep the temperature of the stable 60 to 66 degrees. Put two blankets and a hood on him, bandage his legs, keep the drafts off his feet by placing plenty of straw on the floor. If the horse has a rapid pulse, if effusion is profuse and there is depression, then give him a stimulant.

Give 1 dram of acetanilid every 2-4 hours, according to the size of the horse. Put on regular fever mixture less the aconite with 1 ounce of muriate of ammonia added to a pint mixture. Apply some counter-irritant on both sides of the chest and in front. Provide a nurse day and night and see the horse twice a day.

With the fever mixture and the acetanilid you can reduce the fever in 24 hours. If the mucous glands are severely excited and inflamed, then do not try to reduce it too quickly.

Suppose you have a severe case-temperature 106, pulse 90 , respiration 30, etc. You must stimulate with alcohol, quinine, strychnine, strophanthus and perhaps nitroglycerine. Increase the strength of the heart if possible. We get the best results from moderate doses repeated often.

If you have a valuable draft stallion to treat, mucous rales, pulse 90 and soft, mucous membranes blue, etc., give a dram of 
nux vomica every hour night and day and a dram of alcohol and 20 grains of quinine at the same time.

Alcohol promotes diuresis sufficiently in this stage. It is a good plan to add a little gentian to keep the stomach in order. Give the animal anything that he will eat.

Counter-irritation I am a firm believer in. For this use ammoniacal liniment or mustard.

As to hygiene keep the patient warmly clothed and the stable at the right temperature.

In painful persistent bronchial cough give fl. ex. glycyrrhiza, ammonium chloride, strychnine, strophanthus or digitalis, and an anodine such as heroin (an alkaloid) of which give a grain every 4 hours. Make up a mixture on this basis. drugs.

In prescribing you must know the physiological action of

As regards cats and dogs, they can be dosed according to the breed, size and age of the animal.

Cattle are more subject to fatal bronchitis than horses.

Prognosis.-Bronchiolitis is serious. In acute bronchitis $90 \%$ recover and the other $10 \%$ includes bronchiolitis and suppurative bronchitis. $50 \%$ of bronchiolitic cases recover and $10 \%$ of suppurative bronchitis.

Chronic Bronchitis does not exist very often in horses. It has a peculiar morbid anatomy-a chronic dilatation of the bronchioles, and a chronic irritation of the mucous membranes. This in cattle is so marked, and the exudate is so plastic, that fibrinous tumors forms in the large bronchi. This never occurs in horses, but is found in people and in cattle and predisposes ihe animal to tuberculosis, at least this is the general opinion.

Such an animal is always unthrifty and has a distressing bronchial cough.

Treatment.-There is not very much that can be done. Treat on general principles. Give animal a grass diet. Give a heart stimulant along with

1. Iodide of Potash.

2. Strychnine.

3. Muriate of ammonia.

4. Heroin. 


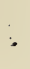



Hydrocyanic acid is recommended by some text-books, but I have not had success with it. It kills by paralyzing the heart.

\section{HEAVES OR PULMONARY EMPHYSEMA.}

This disease is known among horsemen as broken wind. Pulmonary emphysema would seem a paradoxical expression because emphysema indicates that air is present where it should not be; but in this case we have dilated air vesicles and an excess of air in a place where air is normally present.

Nature.-Pulmonary Emphysema is a neurosis affecting the pneumogastric nerve, and incidentally the heart and lungs. In the lungs the air vesicles dilate and sometimes rupture, with extensive destruction of the contractility of the lung substance. Consequently dyspnoea results.

Etiology.-We divide the causes into four classes: 1. Heredity; 2. Breed; 3. Dietetics; 4. Structural changes, dependent upon a previously diseased condition.

It is not difficult to say wherein heredity produces heavesso much so that in localities where stallion inspection is practiced, a "heavy" stallion cannot stand in a public stud, and this is proper. Heaves is unquestionably hereditary. Dr. Baker suggests that this factor may have a relation to the gluttonous appetite of the parent.

Breed is not a very important factor in the etiology. Prof. Robertson claimed, however, that the light draft breed is most subject to heaves.

Dietetics cuts a very important figure. This even plays its part in heredity. A bulky innutritious diet, tends to produce heaves-especially is overripe Timothy hay a very common cause of heaves. All horses living on overripe Timothy do not have heaves, but heredity comes in to determine the percentage.

In structural changes depending. upon a previously diseased condition, the lungs may have had their function partially suspended by pneumonia or bronchitis and the healthy part had to do the work of the whole, in which a chronic dilatation of the air vesicles took place in the healthy part.

In case of dilatation of the bronchioles, the air vesicles at 
the ends of those bronchioles become dilated and the lung is emphysematous.

When a horse with a full stomach is driven rapidly against a strong wind, he is very apt to develop heaves. The patlyogenesis is this: the stomach rests upon the diaphragm interfering with respiration and the horse takes in too much wind and the powers of expiration become tired. The horse takes in more air than his lungs can dispel because the diaphragm gets tired, due to pressure of the stomach.

Nature.-Pulmonary emphysema may be either vesicular or interlobular. Vesicular is seen in the horse in the form of dilatation of the vesicles. Interlobular is seen in cattle in which the air is found in the interlobular connective tissue. The probable reason for this difference is that in the horse there is very little interlobular connective tissue and the walls of the vesicles are stronger, while in cattle the interlobular connective tissue is abundant and there is little or no resistance to the air passing through the vesicular walls into the connective tissue around them.

Pathogenesis and Morbid Anatomy.-In this connection I shall endeavor to show you the connection between cause and effect and how the effect is produced by the cause through the pneumogastric nerve. The pathogenesis will also include the morbid anatomy.

Assume that a horse has an excessive hereditary appetite and is a ravenous eater; eats all that is given him, wants more and is inclined to eat his bedding after he has eaten his feed! The result is distention of the stomach, partly caused by eating more or less innutritious food. Over-ripe Timothy hay is not fit for horse feed. If he has to eat it he should not be allowed to eat more than two-thirds of the straw, this the upper part; the lower third or perhaps half of it is too woody and hard and contains little nourishment. Suppose now that this horse with a ravenous appetite is being fed on overripe Timothy hay,- -he will develop the heaves. He is forced into the habit of having his stomach filled with innutritious food and will soon not be satisfied with the ordinary amount of feed. The result is that the walls of the stomach become distended, 

and this produces pressure on the gastric periphery of the pneumogastric nerves. This is the starting point of heaves. It starts in the stomach. This pressure effects these peripheral nerves, increases their irritability. This disturbed condition of the nerves constitutes what is known as neurosis. The neural disturbance works upwards and forwards through the pneumogastric and involves the pulmonary and more or less the cardiac branches. The pulmonary branch is lessened in tonicity which allows the vesicles to dilate by reducing their tonicity. These vesicles dilate a little more and more every day until finally the dilatation becomes chronic.

In connection with the pathogenesis, especially as it refers to morbid anatomy, notice the results on the vesicle walls. The first affect of the dilatation is anaemia of the vesicle walls. This is a natural mechanical result. The second effect is irritation, and then follows a mild subacute inflammation which runs into a chronic. The only evidence we have of chronic inflammation in these air vesicles is cell proliferation. This produces a thickening of the walls of the vesicles and destroys their contractility. If the early development of the disease is rapid you may get rupture of several vesicles into one large one. Imagine that this is quite general throughout the lungs and you can imagine you are going to have considerable disturbance in respiration. In this pathogenic connection you will 'find that the horse is worse at times; this is due to the condition of the stomach. If a heavy horse gets an unusually big load of food in the stomach you will have increased irritability of the pneumogastric nerves and the pulmonary branches as well. This irritability of the sympathetic system that supplies the unstripped muscular fibres produces spasms of them.

Symptoms.-Heaves may be incipient or confirmed. In incipient heaves you have the disturbed function without the morbid anatomy. In confirmed heaves you have the disturbed function and morbid anatomy. It is therefore difficult in some cases to diagnose a case of incipient heaves. If the doctor can diagnose this he can cure it. Anybody can diagnose confirmed heaves, but nobody can cure it.

The symptoms of incipient heaves are as follows: Breathing 
about 30; nostrils slightly dilated; spasmodic cough tıat is usually dry but without pain; pulse and temperature normal; coughing sometimes in spasms; horse feels well, drives well and eats like a pig-never misses a meal; abdomen good sized, though probably not over large; animal usually in good flesh, assuming that he is liberally fed and exercised every day. If the horse is quiet for two or three hours you can hear a little mucous rattle at the base of the trachea. This mucous rattle indicates irritation of the bronchi or perhaps of the bronchioles.

In confirmed heaves we have these symptoms: inspiration normal; first half of expiration normal; second half requiring additional force which is brought to bear upon the diaphragm by contraction of the abdominal muscles, which throws the abdominal viscera forward upon the diaphragm, pressing the lungs. Concomitant with this is a firm contraction of the intercostal muscles and the second half of the tidal air is forced out by mechanical pressure. Just as soon as this is accomplished the abdominal muscles instantly drop back to their normal position. This produces a flank movement which is characteristic of heaves. If this flank movement is present you can satisfy yourself that the case is one of heaves, that is incurable and that there is morbid anatomy in the lungs. In chronic heaves the cough is apt to be more spasmodic. Sometimes the horse will cough for a minute or two without stopping. During asthmatic exacerbations the nostrils are dilated and the visible mucous membranes frequently become cyanotic. A chronic heaver always suffers more or less from chronic indigestion. This is manifested by a thinness of flesh, pot belly, long coat and general unthriftiness, and by breaking wind while coughing.

Treatment.-The whole treatment should have a bearing upon the pneumogastric nerve which needs its irritability lessened. The following is a good prescription:

$\mathrm{R}$

Fi. Ex. Gelsemium................ 1 dram

Lobelia ....................... 1 dram

Chloral Hydrate (freshly dissolved).... 1 dram

Aqua $\ldots \ldots \ldots \ldots \ldots \ldots \ldots \ldots \ldots \ldots \ldots \ldots \ldots$ pint 


The treatment should follow along the line of a tonic for the nerves.

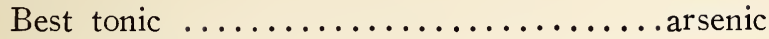

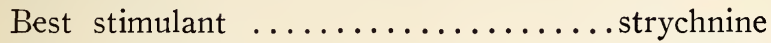

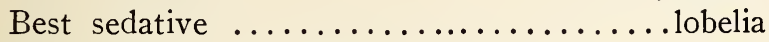

Bear in mind that the horse will eat anything-in fact is inclined to be gluttonous. Therefore give his medicine in powders.

In order to insure that the horse gets the powder, put a double handful of bran in with the oats, dampen it, and mix well. This is the only medicine the horse will require.

R

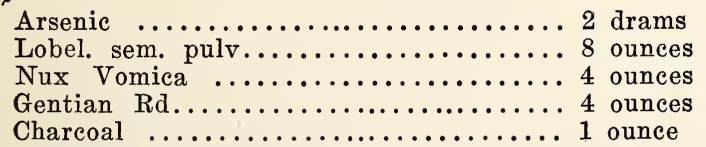

Ft. 60 powders-these will last a month.

M. Sig. -1 powder night and morning in the feed.

Hygiene.-Regulate feed. Give horse a change of hayylimit the amount to $10-14 \mathrm{lbs}$. a day. After he has eaten up his hay put on a muzzle. This is absolutely indispensable. It is a good plan to divide the feed into 2 feeds. If the horse is on the track, take him away and let him rest a month-this gives the air cells time to rest. Give the same amount of grain as usual. The stomach in this case is distended from bulky food, and not from the grain. There is a lot of virtue in bran-not from its nutritive qualities but from its indigestibility.

In 3 or 4 weeks the cough will disappear if the hygiene is regarded. After having one attack, the horse is predisposed to another. This hygiene must be carried out as long as he lives.

Three points I wish to emphasize.

1. That the nature of case is neurosis starting in the stomach and we must treat the pneumogastric.

2. Give a sedative, then a tonic.

3. Then comes the muzzle. Do not forget the hygiene.

Don't imagine that rapid breathing indicates thoracic trouble, and that breathing 30 times a minute and dilated nostrils are diagnostic of acute bronchitis. Do not diagnose a case from the first symptoms or one symptom. In incipient heaves you have 
dilated nostrils without dropping of the flanks. Just as soon as you get morbid anatomy, then you have confirmed heaves. Incipient heaves is curable; confrrmed heaves is not-it all hangs on the morbid anatomy.

Confirmed Heaves.-The treatment practically is the same except you may have to give more of the nerve sedatives and treat the stomach, for it is always in a state of chronic indigestion. Do not give too much arsenic. You can relieve the distress in the breathing in confirmed heaves, but can't cure it. If you have to give Timothy hay, let it be cut early, when it is full in bloom. If you wait until the bloom (2nd bloom) is off, the lower half of the stalk is of no use.

In examining horses for soundness, you must watch closely for the wind of the horse, for dealers have means of covering up the wind by giving alleviating remedies. Watch the action of the flanks; notice how long it takes the horse to recover after being jumped. Normal horse recovers his wind in two or three breaths; a horse may take an half hour-then be suspicious. Catch the horse by the bit and shake him up! Slap him on the ribs, and if he has confirmed heaves he will grunt-then turn hin down.

You can't cover up a roarer with dope so that if he is a whistler, a roarer, etc., the quick jump will bring it out, but the grunt indicates heaves.

If a bovine has heaves, it is no great matter; we are never called to treat it-it is simply a scientific pathological curiosity.

\section{ASTHMA.}

There is a great difference of opinion as to whether horses have asthma or not, but the best authorities recognize it. It is so closely related to heaves that the two are identical with a little difference in symptoms.

Symptoms.-These are as follows:

1. Asthma never arises as an original lesion-animal has heaves in the incipient or confirmed form. 

2. Marked dyspnoea-equally so in inspiration and expiration.

3. Wheezing both in inspiration and expiration. The air goes in and out with difficulty but in heaves the expiration only is accompanied by strong breathing.

4. Symptoms sudderily developed.

5. Anxious countenance.

6. Flanks heaving.

7. Wheezing.

8. Temperature, 102-5.

9. Pulse 60 , or thereabouts.

Diagnosis.-How shall we differentiate asthma from acute bronchitis? The wheeze we never get in acute bronchitis; it is produced by spasmodic contraction of the non-striated muscles in the bronchioles, and this contraction is caused by the suddently increased irritability of the pneumogastric nerve. The pulse and temperature are incidental-not diagnostic, probably due to the dyspnoea.

Treatment.-Nerve sedative is indicated. The sedatives are:

1. Gelsemium.

2. Lobelia.

3. Chloral Hydrate.

4. Belladonna.

Give liberal doses every hour until horse is relieved. 'Also you can give a hypodermic dose of morphine $(4 \mathrm{gr})$ and atropine (1-2 grain) and repeat after 3 or 4 hours.

\section{PULMONARY CONGESTION.}

In its severe form pulmonary congestion is known as pulmonary apoplexy, mechanical engorgement, hemorrhagic infarction (impaction means the same), etc.

Nature.-It is a passive congestion of the lungs-the capillaries and veins are involved. Suppose the capillaries become plugged and the outlet is dammed and the arterioles become involved, as a result we get: 
1. Interrupted nutrition.

2. Dilatation of the blood vessels.

3. More or less extravasation.

4. Sometimes hemorrhage by rhexis.

In case of hemorrhagic infarction, it is never general throughout the lungs, but in patches, which are black and solid.

Etiology.-The most common cause of a typical case is severe exertion when the animal is not in a condition to take it. This applies to horses. A horse "out of condition" is grosshas not had sufficient work. The animal is plethoric and there is an excess of fat in and around the muscles and kidneys, which weakens the muscles, and that around the heart weakens the heart. The bowels are larger and more vascular; the blood ressels are larger and the walls are weaker.

A horse "in condition" to take fast work is in just the reverse condition.

1. Muscles are clearly delineated and hard.

2. Walls of vessels are strong.

3. Contractility of the lung tissue is strong-weak in horse "out of condition."

A horse "out of condition" has a weak heart; a horse "in condition" has a strong heart. The conditioning of a horse to do fast work is a slow process, but he must have regular workwork off the surplus water and fat. Let the horse stand in the stable 10 days, well fed, and not exercised, and then be taken out. Then if he starts out at 12 miles an hour the following symptoms will show up:

1. Horse begins to blow.

2. Slows up in his gait.

3. Short in his wind.

If the driver has any sense, he will let him slow up, but suppose he hits him a crack, and the horse jumps into a quick pace again, then

4. He breaks out into sweat.

5. Slows up again.

6. Looks around-eyes blood-shot. 

7. Anxious countenance.

8. Elbows turned out.

9. Panting 100 a minute.

10. Expired air is cold-air does not reach the capillaries so as to get warm.

11. Ears and extremities cold.

12. Hemoptysis (in bad cases)-bleeding from lungs.

13. Blood coming from the nostrils is frothy.

14. Action of heart is tumultuous-the beats come piling in one after another.

15. Pulse irregular in every respect-frequency, force and rhythm.

In an aggravated case-we do not see it in this countrythe horse dies right away. Such can happen with young bloods, in fox hunts, etc. The horses are at the mercy of the hunters.

So far as other causes are concerned, impure air may cause passive congestion of the lungs-the imperfectly ærated blood would not nourish the tissues properly. This occurs in cold stables which are shut up tight, and the air becomes poor in quality.

Morbid Anatomy.-There is no free arterial anastomosis in the lungs, and the consequences are grave on account of this fact. The infarction occurs in patches, and the circulation there is arrested. If the impacted portion is large enough, the animal will die of asphyxia, but in any case the impacted portion dies, and moist gangrene results: the animal dies in 3 or 4 days. In case the congestion is not severe enough to cause gangrene, then it probably will produce inflammation and end in pneumonia.

These are the four results of pulmonary congestion:

1. Resolution-congestion products become absorbed.

2. Moist gangrene of certain portions.

3. Pneumonia.

4. Asphyxia. 
Post Mortem.-The lesions depend upon the immediate cause of death:

1. Asphyxia-lungs black.

2. Gangrene-lungs green-mortified.

3. Pneumonia (always limited to certain parts) portions of lung will be red or gray.

4. In case of impaction there will be more or less bloody. froth in the tubes. Microscopically there is more or less extravasation of blood into the vesicles.

Hypostatic congestion of the lungs occurs sometimes. The lung is not as severely congested; no bloody froth in the bronchioles; normal crepitation and normal specific gravity of the lung; blood is not forced in-simply due to gravitation; no exudation, no extravasation, no hemorrhage by rhexis or diapedesis. This kind of congestion plays a very important part in diagnosis for forensic purposes.

Treatment.-Give plenty of fresh air; keep him quiet-do not let the horse be ridden or driven home; take him to the nearest stable and leave him there until he recovers. Clothe him warmlly. Give him stimulants-alcohol, strychnine, atropine, aromatic spirits of ammonia, digitalis, strophanthus.

Bear in mind that the congestion is due to diminished cardiac power. It used to be practiced to bleed horses in this condition, but we do not do it any more. The weak heart dictates the rationality of the treatment. Keep the animal quiet for several days and watch for pneumonic symptoms. Clothe warmly, bandage legs, and keep him on light diet. If gangrene develops, great and sudden weakness comes on, loss of appetite and death.

\section{PNEUMONIA.}

Definition.-Pneumonia is an inflammation of the parenchyma of the lungs, i. e., the air vesicles. It is divided into several different classes: 

Location.-

1. Lobar-when a whole lobe is affected.

2. Lobular-when it is limited to lobules. Character-

1. Fibrinous or Croupous.

2. Catarrhal-usually complicated with bronchitis-broncho-pneumonia.

3. Caseous Pneumonia-tuberculosis.

4. Interstitial Pneumonia-when the inflammation is located in interstitial tissue.

We also have (1) Contagious and (2) Non-Contagious (sporadic) pneumonia. Pleurisy is sometimes combined with pneumonia and we get pleuro-pneumonia. The horse practically always has the lobar pneumonia and it is always fibrinous. He has both the contagious and the non-contagious and has the interstitial as a complication in glanders. Occasionally he has the catarrhal form as far as the complication of bronchitis with it is concerned, constituting broncho-pneumonia, but the pneumonia is fibrinous.

The catarrhal form is seen most often in children; fibrinous in horses and men. The catarrhal is usually the form seen in dogs and cats. Caseous is seen in cattle, men and swine.

Characteristics of cut surface of consolidated lung:

1. Fibrinous-glistening, rough, dry, i. e., comparatively.

2. Catarrhal-smooth and moist.

3. Caseous-opaque, cheesy, smooth and lustreless.

4. Interstitial-this is more the inflammation of the interlobular connective tissue, and extends to the vesicles only secondarily. This is the pneumonia which complicates glanders, seen also in coal miners.

Fibrinous pneumonia is usually lobar, while the catarrhal is frequently lobular. Pneumonia may be single or double-right or left lateral. It runs through four well defined stages. 'As- 
suming now that preceding the pneumonia there must be active congestion of the lungs, the various stages are:

1. Dry hot stage of inflammation in which the function is suspended. Walls of the vesicles are dry, and during respiration they crackle and the sound can be heard by auscultation instead of the normal respiratory murmur. Stage lasts 6-10 hours.

2. Stage of effusion-the lung becomes œdematous and will pit on pressure. In the contagious pleuro-pneumonia of cattle the cut lung will drip with serum-never so œdematous in horses. Stage lasts 3-4 days and gradually drifts into the 3 rd stage.

3. Red hepatization-so-called because the lung resembles liver in consistency. The third stage is long or short according to the severity of it. Stage lasts 1-2 weeks and drifts into the fourth.

4. Gray hepatization-this is practically the same as the 3rd stage except that the tissue loses its color-the hematin fades out.

The time of crisis comes at the height of the 3rd stage. This is followed by a progressive softening which runs through the 4th. In recovery the inflammatory deposits soften, liquefy and become absorbed, terminating in resolution in the large majority of horses. More horses recover from pneumonia than any other species of animal. It is marvelous to what extent they recover.

Pneumonia may occur in either one side or the other, either the right or left lateral or both lungs, in which case it is double. We find by experience that it occurs most often on the right side. Right lateral pneumonia is more common than left lateral or double. I think that in this respect it will correspond to the 


observations in the human subject. A German authority gives the following statistics of 6666 cases of pneumonia in the human:

53.70 per cent were right lateral.

38.23 per cent were left lateral.

8.07 per cent were double.

These will run about the same in the horse.

Semeiology.-First Stage-Breathing is accelerated, nostrils dilated slightly, temperature probably 106 , pulse 50 , full, round and strong. Auscultation reveals a faint crackling sound in the lungs; respiration probably 20.

Second stage-By auscultation you hear some crackling and a good deal of dullness; respiration a little faster, probably 24 ; nostrils a little more dilated; visible mucous membranes highly injected and about the 3rd day they begin to become icteric (yellow). The secretions become altered, namely, the urine gets scanty and high colored; feces are scanty; what pellets are passed are coated with slime; thirst increases; expired air hot; tubular breathing increased. In normal respiration you do not hear any tubular breathing; the air passes in and out without any perceptible sound. But in case of rapid breathing, as the vesicles begin to fill, we can hear the air rushing in and out through the bronchioles. This is called "tubular breathing." In normal breathing the only sound we hear is the quiet respiratory murmur.

The symptoms run along from day to day gradually growing worse: temperature always about 106; pulse getting more rapid,-3rd day about $60 ; 4$ th, $64 ; 5$ th, $70 ; 6$ th, 75 ; and 7 th, 80 . The strength of the pulse will depend upon the extent of the lungs involved. If both lungs are involved, the case is usually fatal. A case of double pneumonia that is fatal generally dies on the 5th day. The animal may live to the 6 th, and if he lives to the 7 th, there is hope that the crisis will be passed with safety and he will recover.

Suppose that a fatal case with extensive consolidation has been running for 5 days: on the morning of the 5 th day you find the nostrils intensely dilated, respirations probably $40-50$ per minute, and there is an impulse given to the body by the respiration. You will detect that impulse by placing your hand on 
the hip of the animal. This impulse is in direct proportion to the amount of dyspnœa present. This is a valuable point in making your prognosis. In other words, the greater the impulse, the greater the labor in breathing with disastrous consequences.

The mucous membranes begin to get cyanotic by about the end of the 3rd day. This increases until time of death, when they are livid with an orange tinge. As death approaches, the horse sweats in patches, his extremities get cold, and exhaustion develops rapidly. He persistently stands all through the course of the disease until he drops and dies from asphyxia.

After the lungs have become solid, under percussion you get a solid dense sound,-no sound except the tubular breathing. Note particularly that there is a difference between pneumonia and hydrothorax. In pneumonia you hear the tubular breathing to the very bottom; in hydrothorax you hear no sound below the water line. This tubular breathing is a very important symptom in pneumonia, in which it is a diagnostic symptom. No respiratory murmur can be heard for the air vesicles are full. In such a condition the expired air is usually cold, for it does not stay in the lungs long enough to get warmed up.

Etiology.-A specific pneumococcus causes an ordinary case in croupous pneumonia. In other cases a bacillus is found, which some bacteriologists consider pathogenic for this disease. But these germs are usually found in the mouth of the healthy animal so that it yields to the action of germs which are constantly present in his mouth.

A lowered resistence on the part of the animal will allow the bacteria to grow and multiply. Those exciting causes are as follows :

1. Exposure to cold and wet. If the cold is damp, this aggravates the case.

2. Insufficient ventilation.

3. Defective cardiac power as seen in passive congestion of the lungs.

4. Inhalation of smoke and irrespirable gases.

5. Foreign bodies taken in through the trachea and bronchi. 


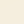



6. Gastric flatulence and eructations.

7. Faulty mechanism of deglutition,-epiglottis does not close over the rima glottis properly, and eructated matter from the stomach falls into the larynx. This occurs in severe nervous prostration in gastric flatulence.

8. Traumatisms.

9. Broken rib-end jammed into the lung.

10. Old age-senile pneumonia.

Pneumonia is a peculiar disease especially as it is seen in oid age. In this condition probably the nervous system is worn out, and we get a weak heart-diminished cardiac power, therefore, being the cause of the congestion and the pneumonia.

Contagious Pneumonia.-We do not know much about its cause. Horses affected with influenza, known as "shipping fever," "sales stable fever," etc., have this pneumonia. This form of pneumonia arising as an incidental feature of influenza is specific-it is contagious. Upon physical examination we find no difference from the ordinary form of pneumonia, but the history is different. I consider that this pneumonia is a local manifestation of a constitutional disease.

Symptoms.-There are two marked symptoms which occur in the contagious penumonia:

1. Great nervous prostration.

2. Marked icterus.

These symptoms are not so marked in sporadic pneumonia. In the contagious the prostrated nerves fail to act in the liver and we get biliary congestion, the bile becomes absorbed and the mucous membranes become yellow. It takes three or four days for this symptom to appear.

Interstitial Pneumonia of Glanders.-This is an incidenial localization of the virus of the disease. It attacks the interstitial tissue primarily, and extends to the parenchymatous tissue secondarily.

Contagious Pleuro-pneumonia of Cattle. - This is specific, usually lobular, but in severe cases may be lobar. In all cases pneumonia in the horse produces a localization of the virus. It is 
fibrinous. When localized, it becomes encapsulated, indicating that the penumonia is sharply circumscribed. Its definite localization is a marked characteristic of the disease. This pneumonia becomes chronic; it never terminates by resolution. In '87-'88 two-thirds of the Cook County cattle died from contagious pleuro-pneumonia.

Symptomatology:-Contagious pneumonia in the horse is usually preceded by a rigor. There is high fever on the first day; temperature 106; pulse full, round, strong and about 50; fine crackling sounds in the lungs; breathing about 22. The second day the temperature is still 106; respirations about 26 ; not so much crackling; leśsened respiratory murmur; increased tubular sounds; part is dull under percussion; expired air is hot; urine is scanty and high-colored; feces scanty; usually complete anorexia. On the third day the temperature is still the same-106; pulse 66; respirations 30; mucous membranes yellowish; nostrils dilated; considerable impulse to the body by respiration; thirst increased; elbows turned out; pellets of feces coated with slime: by auscultation the lung substance is dull, by percussion less resonance. The fourth day (now in the third stage), temperature 106.5; mucous membranes cyanotic; body impulse increased; tubular breathing only (can be heard to the very bottom). Fifth day-temperature lowered, 104.5; pulse 80; respirations 30 ; heaving of the flanks; increased cyanosis; extremities cold; rusty discharge from the nose (not always present); sometimes actual hemoptysis; ears drooped; haggard countenance; expired air cold; pulse growing imperceptible. The animal dies usually on the 5th day. But if he recovers, the crisis is reached on the 7 th day. As the lung clears up you will hear a little respiratory murmur here and there.

Most horses recover quickly from pneumonia, but there will be an occasional one that will linger along for 4-6 months and than die. In such a case the consolidation was excessively hard, so that when it ran into the stage of gray hepatization, instead of resolution, the material broke down and formed abscesses. The products become absorbed and the horse dies from septicæmia. 
- 

Treatment.-There is no specific treatment for pneunonia. Inasmuch as the pneumococcus is recognized as a specific cause, there will probably sometimes be found a serum which will counteract the disease. Now we must treat the symptoms. With the pathology in mind we come to the conclusion that we must stimulate the heart. A strong heart tends to prevent consolidation; a weak heart encourages it. Therefore if you let the heart get weak, the horse will run down rapidly.

Strychnine comes at the head of the list of stimulants. You cannot treat penumonia successfully without it. I have not much use for digitalis-we do not get satisfactory results from using it in treating the horse. It is all right in the disease of the dog and man. As regards alcohol we get good results from its use.

Let us suppose a case in the first stage, the short stage. Heroic treatment may abort the disease. I would recommend either liberal blood-letting or a fever mixture such as acetanilid, aconite, belladonna and spirits of nitrous ether. The acetanilid will act the same as blood-letting, i. e., lower the temperature. Apply stimulants externally, say a liniment. The ammoniacal liniment is too strong to spread over the broad side of a horse; the ordinary white liniment which we use as a sprain liniment is good here. Apply a pint over the surface and then cover it immediately. This warms the animal and seems to act as a special stimulant to the vaso-motor system. Bandage the animal's legs, put him off by himself where he will not have to breathe the air of other animals,-where the air is not impoverished by the fumes of urine and feces.

With this treatment for 24 hours, if you have been successful in aborting the disease, the temperature will have come down to normal, the pulse 50 , though soft and perhaps a little weak.

If the case comes to you in the second stage, pulse 60-70, mucous membrarres yellow, etc., the case is serious. Pulse at 60 is the danger point-you cannot abort the disease. With a view to eliminating the consolidation, carry the horse to the crisis. Cut out the aconite on the second day and potash on the third. Give stimulants. 
The following is a prescription for a serious case in the second stage :

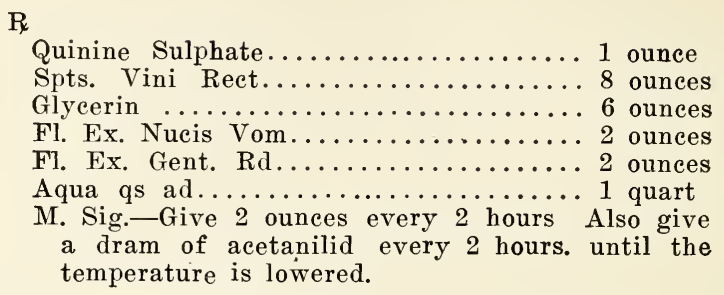

If the horse weighs $1800 \mathrm{lbs}$. increase the dose. Repeat the stimulating liniment night and morning until he begins to get a little sore. Push this mixture until you get the heart to pounding like a hammer.

External treatment in the form of ice packs over the lungs is good for pneumonia. A linseed poultice with mustard mixed in is preferred by some. Woolen blankets wet with cold water can be applied to the chest and covered with warm dry blankets. This abstracts heat and lowers the temperature, alleviating the congestion.

In regard to hygiene avoid drafts, give good bedding, keep cold water before the animal and change often. In lieu of good grass, give scalded oats and bran with a pinch of salt. Give the horse anything that he will eat so as to keep up his appetite. Sometimes a horse will eat corn off the cob when he will not eat anything else. Keep the bowels open,- - soap and water enema once or twice a day if necessary. Keep the temperature at 102-4, but the main thing is the pulse. Keep it at 60 and below and the horse is safe. Watch the action of the kidneys; do not let the diuresis go too far. When sufficient, drop out the potash, the alcohol will maintain sufficient diuresis through the rest of the course. If necessary give a little $\mathrm{Tr}$. Iron and lessen the alcohol. Bear in mind that strychnine is the sheet anchor and hygiene comes next.

If your case is one of broncho-pneumonia, add chloride of ammonia to the fever mixture. This is a special stimulant for bronchial diseases. 



\section{PLEURISY.}

Definition.-Pleurisy is an inflammation of the pleura, a serous covering to the inside of the chest cavity (parietal) and to the outside of the lung (pulmonary). Between the two sides is a partition called the mediastinum. In the horse cribriform openings are in it making a communication between the right and left pleural cavities. The pleura normally secretes serum, which, however, is different from the serum of inflammation.

In the horse pleurisy frequently exists as a separate and uncomplicated disease, but in man and cattle, it often exists in combination with pnemonia. Pleurisy may affect either side (right or left lateral) and it may be on both sides (general). It may originate on either the costal or pulmonary pleura and extend to the other parts by contiguity. In most cases in the horse the costal pleura is the one most usually affected.

Etiology.-There are several causes which come into play:

1. Extension of inflammation to the pleura from contiguous tissues.

2. Direct irritation from local injury or from adventitious growths.

3. Exposure to cold and dampness.

4. Blood contamination. Pleurisy in this case is the localization of a specific disease, e. g., the contagious pleuro-pneumonia of cattle. In horses we find an equally typical case in this localization of influenza.

Extension.-The pulmonary pleura can easily be affected by an inflammation in the adjacent lung tissue, such as we find in pneumonia.

Direct Irritation.-Suppose a broken rib juts into the costal pleura, though not puncturing it. This irritation will set up an inflammation. 'A shaft thrust into the chest of a horse will always cause pleurisy; grapy tumors so common in cattle and hogs will cause it.

Exposure.-Meteorological conditions will cause pleurisy. If 
an animal has a long wet coat and stands for some time, he may come down with an attack of pleurisy in a few hours.

Blood Contamination.-The so-called "sale stable fever" or influenza, may cause pleurisy, but it is general while that due to the other three causes is localized.

Pleurisy may develop from intrinsic conditions within the body, as from anæmia or pyæmia. Hereditary conditions, acute rheumatism or typhoid fever may cause it. It may be incidental to old age. In these latter conditions the pleurisy develops as a complication.

Course.-Pleurisy runs through four well-defined stages:

1. Stage of active congestion.

2. Dry hot stage of inflammation (first)

3. Oedematous stage of inflammation (second).

4. Absorption (if horse does not die).

Special Pathology.-In the first stage of pleurisy, the pleura becomes congested in streaks, spots or patches. These by extension become confluent until the whole pleural surface is bright red. This condition is accompanied by definite symptoms on the part of the animal. These symptoms are very marked and they do not occur in the congestive stage of any other inflammation. The second stage of pleurisy (the first or dry hot stage of inflammation) is characterized by a dry hot pleural membrane whose function is suspended. The costal and pulmonary pleura are both dry rubbing on each other, and by auscultation friction sounds can be detected. The second stage is short, 6-8 hours. The third stage is the second or œdematous one of inflammation and is divided into two parts: A plastic exudation upon the pleural surface (the pleura is so dense that it cannot easily become infiltrated), - a coagulated mass forming a false membrane. In this first part of the third stage pleurisy may terminate by resolution, a sort of fatty degeneration of the false membrane; or if both pleurae are involved, they may adhere and grow together, forming a perfect adhesion, common in cattle and in people, but not often in horses. Recovery may take place after adhesions have formed, but the adhesions remain permanent. The second half of the third stage is characterized by an 


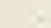



effusion into the pleural cavity, i. e., providing adhesions do not form. This effusion is called hydrothorax-water in the chest. The lungs float up until the animal cannot breathe and he dies from asphyxia. If the extent of œedematous pleura is not too big, and the inflammation subsides before the chest is more than one-third full, absorption will take place and the animal will recover.

The special pathology of pleurisy is just as typical as in any other inflammation. All fatal cases of pleurisy die in the second half of the third stage. If the animal goes on to the fourth stage, the stage of absorption, he recovers.

Hydrothorax is dangerous or not according to the amount of serum in the chest. Its quantity depends upon the extent of the inflammation and its severity. In most cases the liquid in the cavity is serum, but it may be purulent and the condition is called empyema. Empyema is much more serious than hydrothorax.

Symptomatology.-Pleurisy is ushered in with rigor which is more or less severe. As a rule it is less severe than the chill of bronchitis or pneumonia. The coat of the animal is staring and he has a rise of temperature. The congestion of the first stage is painful; the horse is restless, he paws, gets up and down, and sweats. These symptoms are often mistaken for those of colic. This mistake does no harm except that it delays the proper treatment. This stage lasts a few hours when the horse becomes more quiet, he is disinclined to move and breathes with care. His pulse is increased and begins to get hard; ribs are fixed; abdominal muscles contracted, producing a line to the point of the elbow. This depression is called the pleuritic line, which as a rule we do not get in any other disease.

As further symptoms we find the elbows turned out; ears drooping and cold; legs cold and the expired air cold. The horse breathes as little as he can because it is painful, and the air does not reach the vesicles. Auscultation reveals friction sounds over the affected part, like two dry surfaces rubbing together. If you force the fingers between the ribs, it will cause pain and the horse will flinch and grunt. If you move him he will grunt. This grunt in acute diseases always indicates pleurisy in the horse; 
cattle, dogs and men grunt in other conditions. Also in chronic respiratory diseases in the horse we may have a grunt. When pleurisy is complicated with other diseases we can always detect it by the grunt.

The second stage of pleurisy runs along for 24 hours and we find the pulse increased in frequency and hardness. In the first half of the third stage the horse persistently stands; pleuritic line is well marked; breathing increased to 30; temperature 104; urine scanty and high-colored; bowels inactive; friction sounds can still be heard but less on account of the plastic exudate, which lessens the pain as well. $U p$ to this time the animal has eaten practically nothing, but now he begins to eat because the pain is diminished. The inflammation may subside right here and the horse go on to rapid recovery-in 4-5 days. But if the case runs through the third stage, then we get an effusion of serum into the thorax. The pain grows less and the animal will probably lie down and eat a little. The owner thinks that the horse is getting better, but you can see that the eyes are brighter and his nostrils dilated, and the flanks begin to heave. The pleuritic line is lessened, and auscultation reveals increased respiratory sounds above the water line, and a total absence of sound below. The lung floats up on the surface of the water and works harder than usual. As the serum in the chest increases, and as the case has been running 3 or 4 days, œdema can be noticed on the legs as high up as the elbow. Oedematous thickening in the lower part of the chest between the skin and the ribs can be detected. This is a very valuable corroborative symptom. If the pleurisy is complicated with pneumonia, it is not easy to make a diagnosis, for tubular breathing can be heard. If the lungs are consolidated, they cannot float on the surface of the water and it is difficult to diagnose the condition.

Some horses seem to be stronger than others and the œdema does not take place, but as a rule some is present. Also we get œdema as the result of local treatment and this must be differentiated from the pathological lesion.

As the thoracic cavity of the animal fills up with water, notice that the nostrils are dilated, that the eyes bulge; the back arched; horse breathes more slowly; the expired air is cold in 

most cases; with the ear at the nostrils a metallic rınkle can be heard, although this tinkle is not diagnostic. But it is not heard in pneumonia nor in bronchitis. It is a mucous rattle in the bronchi, and is very pronounced.

As the dyspnœa increases the mucous membranes get darker -become cyanotic. The horse dies from asphyxia.

Hydrothorax may be single or double the same as pleurisy. In the mediastinum are cribiform openings through which the fluid can pass from one side to the other. In many cases these openings become plugged up. In such a case the hydrothorax is confined to one side. If the water does not fill the chest more than one-third full, the fluid can become absorbed and the animal recover. If the inflammation subsides during the first half of the third stage the horse makes a rapid and complete recovery, but if the plastic exudate instead of softening forms adhesions, the lungs grow to the ribs.

Symptoms of Pleural Adhesions.-After a horse has made an apparently good recovery and is put to work again, say he goes out driving, when he feels the sudden pain, stops, puts his nose to one side-and forgets all else. He may turn into the ditch, and then go on. This act is due to a stitch in his side, a disturbance of the circulation around the adhesion, and it produces sharp, lancinating pain. The animal does this once and then again. He is a good horse to get rid of.

Treatment of Pleurisy.-If the doctor is called during the chill, he must treat that symptom until he can make a diagnosis as to whether the case is one of pneumonia, pleurisy, etc. Suppose now you have a carriage horse which has had to stand some time after a long drive and upon returning home and going to the stable he soon begins to hang his head, paws around, gets up and down, etc. The coachman gives him colic medicine but it does no good. Upon observing the respirations, you see that they are made with care ; the animal has a temperature of 105-6; his pulse is 50 . He has pleurisy. Adopt heroic measures to abort the trouble; and pleurisy is easily aborted. Put the horse on acetanilid and a fever mixture containing aconite. Apply local stimulants-the ammoniacal liniment, the white sprain liniment, or a sinapism. The value of the external stimulation 
cannot be over estimated-it's ability to abort disease. The liniment must be prompt in its action. By next morning the animal will be all right if you have aborted the case, which can be done 99 times out of a hundred.

If you get a case in the second or third stage, or if your first case does not recover, apply a counter-irritant night and morning. Keep up the fever mixture with aconite to the middle of the third stage. Repeat the acetanilid every four hours in moderate doses. Keep the temperature around 102 if possible, but do not dose too much with acetanilid. Keep the animal warm, feed light, and 90 per cent of the cases will yield to this treatment, and recover by resolution in the first half of the third stage. The other per cent will go on to hydrothorax.

In case of generalized pleurisy "sale stable fever," you may get a horse with hydrothorax after a three days' sickness, while it ordinarily takes 10-20 days for this condition to develop. Pleurisy runs into the third stage very rapidly, but the chest does not fill up with water so quickly. Do not carry the stimulation so far as to blemish the side of the horse, but if you do make this blunder, you can tell the owner that it is better to have a scar on the side than to have a dead horse. You can tell a blister by pinching the skin. If it wrinkles, a blister is there. Open it and liberate any pus that is present, for blemishing is always brought about by leaving the pus and serum there.

While the sides of the animal are soiled with liniment, put a cloth over your face when you wish to auscultate.

Suppose now that you have a case of hydrothorax. Discontinue the counter irritant and the aconite. Give stimulantsstrychnine, alcohol, etc., alternating with tincture of iron. The temperature in hydrothorax stays about 104 . If the chest fills more than one-third full of water you must tap it. This operation is called "paracentesis thoracis."

With trocar and canula evacuate the serum. Some prefer to aspirate the serum, some draw it off with a rubber tube, emptying the fluid under water. Some think that it is not safe to draw off the water rapidly but this is a fallacy. As a consequence none of the trocars are big enough. This one that I use is three-eighths of an inch in diameter and has a short point, 

which is valuable in a case of pleuro-pneumonia. The short point will not be so liable to puncture a solid lung (one such as is characteristic of pneumonia). If the lung is not involved, it will be floated so high in the thoracic cavity that it will be out of the way of the trocar. In the majority of cases tap on the right side between the eighth and ninth ribs. Give the animal one-half pint of whiskey twenty minutes before beginning to operate. This will prevent syncope. Never neglect this stimulant.

Push the fingers down deep between the eighth and ninth ribs and in the pit that forms insert the scalpel, making an incision three-fourths of an inch long. After cutting through the skin, then go through the chest wall-go in slow. The incision should be made close to the anterior border of the rib. Insert the trocar and cannula and withdraw the trocar and the water begins to flow out. While it is running, if the horse shows any resistance, tighten the twitch on his nose-this is all the restraint that is necessary. If the operation is done between the seventh and eighth ribs, the elbow is apt to interfere, if the animal is in pain and restless. Remove the twitch as soon as the flow is established. If there is any coagulum in the fluid, keep the cannula pervious with a small blunt instrument. The horse will improve at once with this operation.

If the cribriform openings of the mediastinum are plugged, you may have to tap the other side. Wait a day or two before performing a second operation. Do up the wound with a dry dressing and keep on for a few days.

In some cases you may have to tap again, but do not operate in the same place; make a fresh opening. Never open up the old sore.

Prognosis.-This is according to the color of the serum; if amber colored, it is favorable; if red, unfavorable.

In case of a purulent liquid, clean out the chest cavity with 2-3 gallons of tincture of iodine-1 per cent solution. Attach a rubber hose to the cannula, and run the solution (temperature 102 ) into the chest from a fountain syringe. Hold there for 10-15 minutes and then let it run out again. In the human two 
or three openings are made and the cavity is flushed out with a solution of bichloride of mercury.

Pneumothorax.-In case of puncture or wounding of the thoracic wall, air may get into the chest. This is dangerous and will kill the animal if the pressure is sufficient to interfere with the action of the lungs. In such a case stop up the opening as quickly as possible.

\section{RECORD OF A CA'SE OF PNEUMONIA:}

HISTORY.-Case of a bay horse, 8 years old. He was taken sick one forenoon while standing in the railroad yard waiting for the milk train to come in. He came down with a chill soon after reaching home. Dr. Baker was called to see him the next day (13th).

The following is the record of his pulse, temperature and respiration for the two weeks of his sickness:

Date. Temp. Pulse. Resp.

\begin{tabular}{lcccc}
13 & End of 1st stage & 104.8 & 60 & 24 \\
14 & 106.2 & 66 & 30 \\
15 & 2nd and 3rd & 105.4 & 72 & 32 \\
16 & stages & 105.6 & 70 & 36 Crisis \\
17 & 4 th stage begins & 104.4 & 68 & 48 passed. \\
18 & Gray hepatization & 102.6 & 50 heart be- 32 \\
19 & \multicolumn{4}{c}{ gins to pound32 } \\
20 & 102.8 & 56 & 32 \\
21 & 103.5 & 58 & 20 Nervous \\
22 & 102 & 50 & 32 system \\
23 & 101.6 & 47 & 33 only mod- \\
24 & 102.2 & 48 & 30 erately \\
25 & 101.6 & 48 & 22 & excited \\
& 100 & 37 &
\end{tabular}




TREATMENT.-The following was the dosage:

$\mathbf{R}$

Quinine ................... ounce

Nux Wom. .................... 1 ounce

Fl. Ex. Gent................ 1 ounce

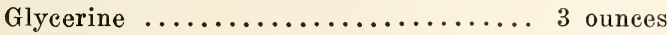

Alcohol ...................... 4 ounces

Aqua qs ad................... 1 pint

M. Sig.-One dose, 2 ounces every 2 hours. Also give 1 dram of acetanilid every 4 hours.

On the 23rd day the quinine was stopped and ammonium carbonate (1-2 dram) was substituted for it in the mixture, the alcohol being lessened to 3 ounces. The acetanilid was discontinued after the fifth day.

\section{DISEASES OF THE DIGESTIVE SYSTEM.}

\section{ANATOMY AND PHYSIOLOGY.}

\section{(Horse.)}

The lips are the prehensile organs, the incisor teeth are the nippers. The food passes back on the tongue, and while the animal is chewing, the salivary glands are stimulated to secrete enormous quantities of saliva, which is poured out partly to moisten the food and partly to digest it. Its digestive action is amylolytic, i. e., digests starch. It takes about 15 minutes for the saliva to act. The food is then formed into a bolus, which passes down the œsophagus to the stomach.

In all monogastric animals the stomach is in a collapsed condition before eating begins; in ruminants some food is always present in the stomach. In monogastrics the stomach is no larger than the amount of food put into it. After the food enters the stomach, the starch digestion goes on for a little time, and some lactic acid is formed. The presence of the food stimulates the gastric juice to flow; this juice contains hydrochloric acid, and when its secretion is fully established, the starch digestion ceases. As soon as the acidulation comes up to a certain point, the pylorus opens and the products of digestion (now called chyme) pass out into the duodenum, where the bile 
and pancreatic ferments act upon it. The action of the bile is three-fold :

1. Changes the acid reaction to alkaline.

2. Emulsifies the fat.

3. Acts as a laxative.

The pancreatic juice contains four ferments:

1. Amylopsin-to digest starch.

2. Steapsin-to digest fat.

3. Rennin-to digest milk.

4. Trypsin-to digest proteids.

In the duodenum the chyme is changed to chyle.

In the intestine the food meets with succus intericus, which Pawlow calls the "juice of juices." The food is absorbed by the villi of the small intestine. In the villi are the small lymphatics which take in the chyle, carrying it to the receptaculum chyli and from there on through the thoracic duct to the heart; the villus capillaries absorb the other products of digestion and carry them to the liver.

The stomach is the fountain of health; if in good condition the animal is well. In man indigestion is the main factor causing disease and this is also true in the other animals. The food is either too bulky, or too concentrated; too rich or too poor; too wet or too dry; contains too much indigestible matter or not enough of it; the animal is either over-fed or not fed enough; he is fed at too long intervals or irregularly.

As regards the tendency of digestive organs to develop disease, this depends largely upon the parts of the digestive system most often in use: such parts will be most abused. In the horse the food is a short time in the stomach and a long time in the bowels. Consequently the horse has less disturbance in the stomach than in the bowels-diseases of the bowels are common. In ruminants the reverse conditions attain-diseases of the stomach are common and rare in the bowels.

The horse, not being a ruminant, should eat slowly and masticate his food thoroughly. It is important that the amylopsis in the mouth should be complete. Many diseases come from bolting the food. Sometimes it is difficult to prevent bolting. Too concentrated food produces diseases of the stomach; tou 


bulky food, diseases of the bowels. It is a recognized fact that wholly digestible food produces paralysis of the stomach. For this reason it is not a good plan to feed a horse on flour: the stomach requires the cellulose covering of the grain to irritate its glands. Nature provides this indigestible matter for an irritating factor and consequently the stomach will not work on too concentrated food. If such is given, the germs present in the stomach take advantage of its inertia and set up a fermentation which leads to gastric flatulence and possibly rupture. On this account some object to cooking the food, but this does no harm if plenty of indigestible stuff is given too. Suppose you scald the oats, this makes them easier to digest and also renders part of the cellulose digestible as well; but you cannot feed the horse the rolled oats which people eat, for it is too concentrated.

Do not clip the oats for the horse, he needs the hull as an irritant. Clipped oats make work for the veterinarian.

Barley, wheat or other heavy grain is too concentrated food -it has not enough indigestible cellulose. The horse needs hay. Without it the stomach will not work.

\section{PHENOMENA OF THE DIGESTIVE DISEASES.}

The phenomena of Digestive Diseases are fewer than those of the respiratory system but these phenomena are well marked. They are as follows:

1. Flatulence.

2. Colicky pains.

3. Turning up of the upper lip-expression of nausea. The horse stands still, suddenly stops eating and then turns up his upper lip. The only exception to this being an evidence of nausea is in the case of a stallion teasing a mare.

4. Constipation - pellets hard, small, scanty, or wholly absent.

5. Diarrhœa. 


\section{DISEASES OF THE MOUTH.}

\section{CONGESTION OF THE BUCCAL MEMBRANE.}

Definition.-This condition is a congestion of the mucous lining of the mouth. It occurs in young animals during teething. When a temporary tooth drops out and a permanent one comes in its place, this produces a circulatory disturbance. The buccal membrane may also become congested as the result of gastric indigestion.

"Lampas" is really an imaginary disease. It is nothing but the congestion of the bars of the mouth. The bars hang down usually on a level with the upper incisors, sometimes below in old horses. Disease of the bars never occurs except in puncture or injury. If a horse does not eat, a quack may be called to look into his mouth; he sees the bars projecting down, and as a bluff he says its the "lampas." Or the horse falls into the hands of some horse-shoer, who says that the bars must be turned out. This is cruelty to animals. The bars in a horse's mouth are for the purpose of keeping the oats inside the mouth while being chewed.

You cannot ignore the owner's diagnosis of "lampas." Take your knife and scarify the first bar about 1-3 of an inch deep. Then give the animal a mouth lotion, bicarbonate of soda, or borax and charge your client $\$ 2.00$ ! If your client will permit you, examine the animal further to find the reason of his not eating.

Dentition and indigestion are the two main causes of sore mouth. In children, puppies and pigs, convulsions may occur as a result of the nervous disturbance accompanying the eruption of a tooth. This can be relieved by scarifying the gum.

Hemorrhage of the Palatine Artery.-The palatine artery is often wounded by empirics who try to scarify the bars in case of the so-called lampas. The tissues around the artery are very firm and do not contract down upon it when wounded as with other vessels. When such a condition falls into your hands, put on your overalls and gown, for the blood will spurt and pour out profusely. Take a tightly rolled wad of a substance like oak- 


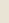



um or cotton (about the size of a goose egg) ; dip the end of the wad in Munsen's solution of iron and apply it to the roof of the mouth. Holding up the upper lip, wind the oakum tightly to the incisors 6 or 8 times, and then leave it alone. It will be all right the next day. Give the owner a lotion to wash out this horse's mouth and charge him a good fee!

Do not embarrass the horseshoer by running down his work before the owner. As far as possible protect the horseshoers.

\section{STOMATITIS.}

Stomatitis runs a course through three kinds of inflammation: (1) Catarrhal (2) Vesicular and (3) Pustular. Further we recognize two kinds, the contagious and non-contagious.

Etiology.-The contagious form of stomatitis is produced by some specific germ, which as yet we do not know. The noncontagious is often due to indigestion; it sometimes occurs through local injuries and infection of wounds. Very fortunately for all animals the infection of the mouth wounds is not common. The wounds are common but their infection is not. 'The salivary wash of the mouth is one continual disinfectant; the saliva washes the germs away and bathes the wounds.

Semciology.-We see stomatitis in foals and in calves from too much nursing. The foam at the side of the mouth of a little calf, when in excess, produces sore mouth. This is "baby's thrush." A little gastric indigestion may accentuate it. Acari may infect these sore places. The form seen in foals is the catarrhal form. It will show itself in deep red patches, on which a bran-life scurf will form. This is coagulated mucous, a plastic exudate, and it soon gets fetid. The feter is due to the degenerated mucous. In a bad case these patches extend and coalesce so that the whole lining of the cheek may be involved, even that of the lips and the frenum lingui. The roof is not often affected. The catarrhal form occurs later in the fall-the lips of the horse get chilled when he is grazing on the frozen grass in the late fall much worse than in winter.

In the vesicular form of stomatitis the patches are covered with little vesicles. These vesicles are probably due to indiges- 
tion; they are little cankers, coming up first as vesicles then discharging and ulcerating. The pustular form may follow the vesicular.

The technical name of the pustular form of stomatitis has been given by Prof. Williams as Stomatitis pustulosa contagiosa. It has an incubation of about 3 days. It will likely show as a profuse eruption on the lower lip, sometimes extending around on the outside of the lips even to the outside of the cheeks, down the shoulders and breast to' the hoofs. The specific germ has never been found. The course and termination are the same as equine variola, which in my opinion is the same disease. Horse pox is very contagious to other animals and to people. Never give a horse a ball or do dentistry on him without examining his mouth for contagious stomatitis. If the saliva from such a case drys on your hands, they will become inoculated with infection. If this does occur, soak the hands in a 5 per cent solution of formaldehyde.

Treatment.-In the non-contagious form the first thing to do is to remove the cause. Change the diet of the animal if necessary. If the disease occurs in a nursing animal, do not let him nurse continually. Separate him from his mother. Wash the udder and teats with borax and also the mouth of the foal. In adult horses nothing is better than borax.

As to the contagious form isolate the animal. It is no use to take him out of the stall, you must isolate the stall. If the partitions are board, they are all right, but if wire, board ones must be put up. Put ropes or chains behind the horse to keep him in. However, he can be used in single harness if he is not tied to a post or watered at a public watering trough. If his bit is used for other horses it must be disinfected. The inflammation must run its course but chlorate of potash, a saturated solution, will help.

\section{GLOSSITIS.}

Definition.-This is an inflammation of the muscular substance of the tongue. It is usually due to local injury. It may be produced by scalding doses. A puncture produces an inflamma- 
. 

tion of the intimate lingual structure, but the scalding dose produces also a catarrhal inflammation of the mucosa.

Cause.-The cause is invariably due to local injury. In cattle it may take on the form of actinomycosis, tuberculosis, etc. The horse is not often affected with actinomycosis and tuberculosis is rare.

In glossitis the tongue swells and protrudes between the incisor teeth. The mouth hangs open and the tongue hangs out. Deglutition is difficult; the horse is unable to eat and he may be unable to drink. Constitutional disturbances may occur.

Treatment.-Ascertain the cause and remove any foreign body. If a puncture is present, follow it to the bottom with a probe-pointed bistury and enlarge the opening. Irrigate well with an antiseptic; if a large wound, bathe with an antiseptic solution. Use water as warm as the horse can stand, carbolized 1 per cent or a 10 per cent solution of boracic acid or a saturated solution of chlorate of potash. If the swelling does not yield promptly, scarify the tongue on the side an inch long and 1-4 inch deep. Bathe the tongue with water 102-3 degrees F. to promote bleeding and then go on using the antiseptic solution. If the mouth gets foul, sponge out twice daily with permanganate of potash.

If the tongue is injured by getting caught in a halter chain, and is torn, say three-fourths off, amputate the balance. You cannot get union between incised or lacerated surfaces in the mouth. In examining a horse for soundness, always examine the tongue. A horse with a short tongue is damaged and he cannot feed so well.

\section{PAROTIDITIS.}

Parotiditis is the inflammation of the parotid gland. It is usually acute and it usually goes on to suppuration, forming one large abscess or two or more small ones. The cause may be influenza or strangles, or some other blood contamination. It may be due to local injury. In my experience the disease occurs without any ascertainable cause.

Symptoms.-The parotid gland swells and is very sore; the 
nose is poked out; deglutition is difficult; neck stiff; and it is hard for the animal to eat. The abscess is likely to be deepseated, and will take a long time to rupture spontaneously. Consequently we do not wait but open it up. Small superficial abscesses may rupture of their own accord.

Treatment.-Hot poultices are easier than applications of hot water. If you set a man to bathing a part with hot water, he in inclined to get tired and stop before he has accomplished any good results. A hot linseed poultice will soften the abscesses and about the fifth day it can be opened and the pus let out. Into the cavity inject an antiseptic solution, then insert a little pledget of gauze and poultice some more to soften the inflamed swelling. If a poultice is properly made with boiling water, it must be pretty nearly sterile. You never get any infection from a poultice, if properly made.

Usually the salivary discharge from the gland is increased and a salivary fistula will follow. This discharge is clear and odorless. Insert a probe-pointed bistury into the fistula, enlarge the opening slightly-to about $1-4$ inch in diameter-and inject tincture of iodine full strength. A single dose ought to cure. If not, repeat about once in every ten days.

\section{PTYALISM.}

Ptyalism is an increased flow of saliva. This cannot be considered a disease but is a symptom of some other trouble. Ptyalism accompanies many diseases, such as sore throat, tetanus, sore mouth, etc. As a rule it may be said that any inflammation near the salivary gland will increase the secretion of saliva.

Treatment.-The most effectual treatment is to remove the cause. When the horse slobbers too freely while in harness or while eating, there is something wrong with his mouth-either the bit irritates him and causes soreness or his teeth are too sharp and the bit pulls the cheek against the sharp corners of the upper molars. It is not at all uncommon for a horse to foam at the mouth a good deal while driving. This, of course, is disagreeable and looks bad. When the veterinarian is consulted he 

naturally expects to find something wrong with the mouth. We usually find that the bar of the curb bit (the usual bit for coach horses in order to give them proud carriage) does not fit the mouth or jaw; and for that very purpose, to make the horse prance and make him showy, the coachman has the lines buckled down in the curb and touches him up with the whip. Occasionally the horse gets crazy, especially a young horse, under such irritating conditions. The horse pulls hard on the bit, and the bar bit with the chain underneath is brought back against the sharp edge of the jaw, it cuts through the tissues, and injures the bone. The contused bone dies and sloughs off. This condition makes a very sore mouth, therefore always look for the cause.

The horse that slobbers too freely while eating cannot be cured unless you can find some mechanical cause, as the sharp molars. If you do, round them off, especially the front molars where the bit pulls against them. It may be necessary to change the bit for a time; get a bit that will bring pressure on the sides of the bone instead of on the bar. Recommend a chain bit covered with leather or rubber. A straight bar bit is an abomination in a horse's mouth, such a bit should never be used.

\section{SALIVARY FISTULA.}

The duct of Steno carries the saliva from the parotid gland to the mouth, passing down on the inside of the lower jaw, rounding the bone to the outside of it and passes upward on the edge of the masseter muscle and empties into the mouth opposite the third upper molar. In a fistula this duct becomes opened-it is difficult to say exactly what does open it, but it is so exposed to injury by blows of various kinds-from kicks, from rubbing on the manger, etc., that it is probably opened in this way.

Treatment.-Establish an opening between the fistula and the mouth through the natural passage. To do this pass a stiff silver-plated probe, a small one first to establish the opening into the mouth, then use a larger one. By increasing the size of the probe in the course of four or 'five days you will be able to pass a sound which is at least a quarter of an inch in diameter. Then put a speculum into the mouth, but do not open too wide; 
pass the hand into the mouth with the front of the hand against the cheek and feel the probe as it comes up.

Suppose the accident came from the kick of another horse on the cheek. The main damage, was a bruise on the jaw bone. This resulted in inflammation, exudation, organization and finally complete obliteration of the duct. This is frequently the case. We find that the organized lymph is not as tough as the wall of the tube and with pressure you can work an opening through into the mouth, a small one anyway. Take a brass or silver probe and run a piece of tape through the eye of it, drawing it into the mouth from the outside. Leave it there three days, then remove it and syringe it out. Clean the outer surface around the opening, scarify the edges of the fistula freely and trim it out to make a raw edge. Put a continuous suture around the opening and draw it up like a draw-string, using a solution of collodion to wash it. Do not give the horse anything to eat; tie his head up for about three days and give him plenty of water. This reduces to the maximum the secretion of saliva. After three days give him a little fine hay, letting him eat it off the floor. While he is eating the saliva will run out through the mouth. If this treatment does not succeed, then put on a fly blister. This stimulation will produce a local inflammation and increase the repairing process. Repeat if not successful the first time. Some surgeons recommend to destroy the parotid gland in bad cases, but this cannot be condemned too strongly. It is very painful and produces very unsatisfactory results.

\section{SALIVARY CALCULI.}

Salivary calculi are calcareous concretions which are found usually in the duct of Steno on the broad side of the cheek about opposite the lower molars. Probably every calculus has a nucleus, some foreign body which has worked itself into the duct of Steno through the mouth. It may be a little piece of barley or a wheat beard, hull of oats, buckwheat, etc. If this happens it seems never to get out and produces a little local irritation, enough to change the character of the secretion in the duct. The salivary salts accummulate around and on the body and finally 


the calculus is formed. These calculi are heavier than those of any other part of the body. Their increased density is due to phosphate of lime. Also they are slow-forming calculi. They often obstruct the duct to a considerable degree and the part of it nearest the gland becomes distended.

Treatment.-Remove the calculus by the aid of the knife, always operating through the mouth, never through the skin. Probably no after-treatment will be required. Sponge the wound three or four times a day with a solution of borax and impress upon the owner that it is a serious case; it is, however, a simple one!

\section{DISEASES OF THE THROAT.}

\section{PHARYNGITIS.}

Pharyngitis is an acute inflammation of the pharynx. It is a catarrhal inflammation. Many cases of sore throat are cases of pharyngitis. Usually a combination of laryngitis exists with the pharyngeal inflammation, but either may exist alone.

Etiology.-Pharyngitis may come on with sudden exposure to cold and dampness. It may be caused by foreign bodies, acrid substances in the food or medicine, etc. Sometimes the inflammation is so severe as to produce a post-pharyngeal abscess.

Semeiology.-The symptoms run as follows:

1. Difficult swallowing.

2. Possibly a little fever.

3. Respirations normal.

The symptoms in pharyngitis are much the same as those of laryngopharyngitis.

Treatment.-In this condition counter-irritation is indicated. Give anodine or a stringent medicine with a syringe. Either give an electuary on wooden spatula or the iron gargle with a syringe. Never drench a horse in case of pharyngitis. The iron gargle is good. It consists of tincture of iron one ounce and chlorate of potash $1 / 2$ ounce to the pint of water. The dose is 2 ounces. 


\section{DISEASES OF THE OESOPHAGUS.}

\section{OESOPHAGITIS.}

Oesophagitis is the inflammation of the oesophagus.

Etiology.-This condition is caused by an extension of inflammation from other parts; by the scalding of the mucosa with caustic drenches; by external injury, kicks, etc.

Semeiology.-The symptoms may show a rise of temperature; difficulty in swallowing is present; disinclination to eat.

Treatment.-In an ordinary simple case if you remove the cause, the animal will recover. Give a little antiseptic medicine. A solution of borax, bicarbonate of soda, a mild dose of permanganate of potash, etc., are good antiseptics.

Sequelae.-Stricture of the oesophagus may follow its inflammation. The result is a thickening of the wall with a lessening of the lumen, which in bad cases may amount to stenosis. The wall may be thickened 3-5 times and the lumen not more than $1 / 2$ inch in diameter. The animal cannot swallow. Even if the lumen is an inch in diameter, the food will go down with difficulty, although water may be swallowed easily. The food accumulates above the stricture and this occasions frequent choking.

Treatment.-Sloppy food or milk is indicated. Take away the solid food. The stricture and dilatation are incurable, and they occur oftener than we imagine.

In case of oesophagitis from local injury, there is more or less enlargement in the outside tissues and this causes choke. If the tissues of the oesophageal wall are not destroyed, the case will reeover. Injuries from a kick may press the oesophagus against the vertebrae and actually destroy the tissues of the wall without even wounding the overlying skin. Eventually an elliptical slough may occur; the surrounding tissues swell and become doughy. Open up the tissues over the wound and if such is the case, destroy the animal. But if the wall of the oesophagus is only wounded, sew up the lacerated edges, turning them in toward the lumen of the oesophagus. If they are left out, they will not adhere. Openings into the oesophagus are usually fatal. 

Following the operation, keep the animal's head up for a time and feed him on milk and water.

\section{OESOPHAGISMUS.}

Oesophagismus is a spasmodic condition of the oesophagus. It is a functional condition purely nervous. You can see the effects, but you cannot foresee the condition. Usually the spasms subside after a few minutes.

It is caused by something being swallowed that irritates the nerves, and produces spasmodic contraction upon the bolus. In view of this never give a.dry ball, always wet it.

In order to remove the obstruction put the fingers at the bottom below it and move it up towards the mouth. If the obstruction will not dislodge, you may have a case of choke.

Sometimes coachmen get the idea that raw eggs will make the horse's coat glossy. They even send them down whole, shell and all. This frequently causes choke. Oesophagismus is a symptom of choke. But if the cause of the irritation is in the thorax, there will be little eating and then vomition. The food may be streaked with blood.

Treatment.-Give a liberal dose of morphine. This blunts the nerves. When the animal is thoroughly under the influence of the drug, then give two or three doses of cottonseed oil. If the oesophagus does not relax, the animal will regurgitate the contents of the oesophagus. Then pass a probe and push the obstruction on into the stomach. Do not wait until the inflammation starts up before doing this.

\section{CHOKING.}

Definition.-Choking is obstructed deglutition by the interrupted passage of any substance from the mouth to the stomach. The regions of choke are divided into three sections, cervical, thoracic and cardiac.

Etiology.-Horses may choke on dry feed. They are ravenous eaters-they bolt their food without proper insalivation. Cows usually choke on pieces of apples, potatoes, carrots, sec- 
tions of turnips, etc. If the cutter is out of order, the food will be imperfectly cut up and the large pieces will cause choke. Cows will choke on apples which they have picked up from the ground. Cows which are giving milk are inclined to chew bones and linen garments,-I have known them to chew up a whole washing and get choked! Dogs usually choke on spools, needles, hatpins, etc. Cats the same. Sheep and pigs seldom chokethey are not so ravenous. Poultry fed once a day and then given meal will eat too fast and choke. Horses occasionally choke on solid substances.

Semeiology.-The horse quits eating and makes spasmodic but ineffectual efforts to swallow. He becomes restless; moves back and forth; has an anxious countenance, etc. Nothing can produce more nervous excitement than a choke. In the course of a few hours the obstructed œsophagus produces nauseaanimal will stretch his neck and he gets spasmodic cramps of the cervical and pectoral muscles. These draw their heads toward their knees. This is seen also in gastric indigestion. It is simply a diagnostic symptom of nausea, and may be so severe as to cause the horse to shriek with pain. These cramps are called retching or ineffectual attempts to vomit.

If the choke is a cervical one there will be an enlargement along the course of the œsophagus on the left side of the neck about the first rib. In dogs, cats and chickens the choke is cervical; in cattle the choke is often thoracic; in the horse and in cattle the choke may be at the cardiac opening. The symptoms of thoracic choke are much the same as in the cervical but the nausea is more marked. The horse fills up his œsophagus to the obstruction and then throws out the food again. He throws it out through the nose. This is an act of vomition and there is also reverse peristalsis. In cardiac choke the obstruction cannot be seen until the œsophagus becomes filled, and then the obstruction is seen in the cervical region.

In cattle any choke leads to tympanitis of the rumen. There is a great slavering of the mouth, present in all animals, however. Dogs, cats and people have violent fits of coughing, but not the horse. If the choke is not removed, it acts as an irritant and produces inflammation running through the first and second 

courses and terminates in gangrene. The animal dies from septicæmia.

As regards the horse the conditions producing choke are as follows :

1. Oesophagismus.

2. Oesophagitis - especially a factor when the inflammation runs to ulceration.

3. Organic diseases of the gullet in the form of a stricture, frequently following oesophagitis.

4. Diseases of the salivary glands, by which the quantity of saliva is diminished. It is impossible for the food to go down the oesophagus without being thoroughly insalivated.

5. Voracious appetite-the horse bolts his food and it enters the oesophagus dry. Deranged or decayed teeth interfere with mastication.

Treatment.-The first thing to do is to give a hypodermic injection of morphine- 4 grains. As a rule it is impossible to move obstructions downward, but they can be moved upward. Give an internal dose of cotton seed oil (about 2 ounces) with a syringe. Then manipulate the obstruction rubbing toward the throat. The animal will throw out the oil, and then give another dose and repeat. You can put a hose through the nose of the horse and turn the water on, thus washing out the obstruction at times. This surgical operation includes exposing the oesophagus and passing a ligature around it with the stomach tube inserted to prevent the ingesta from falling into the larynx. Before attempting this operation read up on it.

Cattle often choke in the cardiac region. The proper thing to do is to place a speculum in the mouth (in case the choke is in the cervical region) and let some one press upwards beneath the obstruction, while a small hand goes in and gets it. Or a probe can be passed and push it down into the stomach. 
In severe cases of tympanitis accompanying choke in cattle you may have to tap the rumen.

In choke in dogs and cats, hold the mouth open and go after the obstruction with a pair of forceps.

In the chicken give them a dish of water and they will help themselves.

\section{ORGANIC DISEASES OF THE OESOPHAGUS.}

An organic disease of the oesophagus is the change in structure of the wall caused by a stricture. The causes of a stricture are:

1. Irritation.

2. Congestion.

3. Inflammation.

4. Organization.

These changes occurring in the wall lessens its tube lumen, thickens the wall, and destroys its dilatibility. Immediately anterior to the stricture there will be a dilatation; the older the case the larger this will be. In connection with the stricture is more or less degeneration of tissue.

Symptoms.-There are frequent choking, slow deglutition. contents of the dilatation frequently thrown out and occasionally streaked with blood.

Prognosis.-The prognosis is unfavorable because you cannot dilate the stricture nor reduce the dilatation. The ultimate end is oesophagitis with a choke that cannot be removed.

Treatment.-Drench the animal with small doses of something that will tend to cure the inflammation-saturated solution of borax, chlorate of potash or sodium bicarbonate. These should be given several times a day. Give the animal sloppy food and compel him to eat slowly.

In order to make a horse eat slowly put stones in his feed box, and these will hinder him from getting his food so fast. If this fails there are patent boxes which can be used for this purpose. 



\section{DISEASES OF THE STOMACH.}

\section{VOMITION.}

Vomition is called emesis or vomiting. The emetic center is in the medulla. Something disturbs this center, and the reflex action of the disturbance is transmitted to the stomach. The symptoms of vomiting are:

1. Depression.

2. Turning up of the upper lip.

3. Abhorrence of food. The nausea increases until we get a reflex peristalsis of the stomach, duodenum and sometimes of more of the small intestines.

The act of vomiting is as follows:

1. Deep inspiration.

2. Glottis fixed.

3. Spasmodic forcible contraction of the abdominal muscles.

4. Food thrown out through the nose.

5. Mouth in all animals except the horse wide open.

The stomach cannot empty itself without the aid of the abdominal muscles.

Horses do not vomit except under peculiar conditions; carnivora vomit easily; omnivora comparatively so; and herbivora with great difficulty. Cattle do not vomit because the rumen is large and comparatively insensible. As a whole the ox is phlegmatic. Horses vomit with difficulty on account of the shape of their stomach. At the œesophageal entrance there is a fold of mucous membrane which acts as a valve and serves to keep the food from going back toward the mouth. Except in case of a distended stomach vomition cannot occur. It is usually accom panied with gas. There are four conditions under which a horse vomits :

1. Stomach distended with gas and food mostly liquid. 
2. Organic disease of the œsophagus with a dilatation of the cardiac opening.

3. Rupture either partial or complete of the walls of the stomach. The rupture may be $2-4$ inches long; if it is very long, the horse cannot vomit.

4. Closure of the pyloric orifice by a plug of indigestible food which prevents the food from passing out into the duodenum.

In case of distention the greater curvature will be the most distended and rupture will occur here.

\section{INDIGESTION.}

Indigestion occurs in all animals. It is a functional disturbance. It is named gastric or intestinal according to its location. The gastric form occurs in three degrees of severity:

1. Acute.

2. Subacute.

3. Chronic.

Under the head of acute indigestion we have two or three different conditions :

1. Engorgement of the stomach.

2. Stomach staggers.

3. Gastric flatulence.

Acute gastric indigestion usually comes on suddenly. It is always a serious affection, often producing death either by (1) exhaustion or collapse, (2) asphyxia, (3) rupture of the stomach, or (4) gastritis.

Etiology.-The chief causes are overloading the stomach, eating too fast, insufficient mastication, insufficient insalivation, eating when physically exhausted, and improper food, especially that not assimilable by the animal. The insalivation is very important because it produces amylopsis. Eating when physically exhausted is probably the main cause of indigestion-the horse will eat too fast and the stomach, being depressed, will not act. 

Do not give a tired horse oats. Let him eat hay for a half hour and then give him the oats. Too much bulk (if very indigestible or too concentrated) will cause gastric flatulence.

Symptoms.-Suppose we have a case of gastric flatulence from bolting the food. The following are the symptoms:

1. Restlessness - the horse lies down, gets up soon, turns around; the worse he gets, the shorter time he lies down. When tympanitis is present, he cannot lie down.

2. Mild colicky pains.

3. Animal looks around, most often to the left.

4. Trunk more or less distended. Flanks fuller than normal, but not very tympanitic. The distension is more under the ribs. In intestinal flatulence the distension is more in the flanks.

5. Horse sweats.

6. Eructations-positive evidence of gastric flatulence. It is accompanied by more or less sound, which may be so slight that you cannot hear it without putting the ear to the neck. These eructations give the animal great relief, but all cases do not eructate.

7. Nausea-retching and painful vomition, especially in the horse. As a symptom of the retching we get a spasmodic contraction of the muscles of the neck and breast the same as in choke. Retching is a painful effort to vomit without succeeding.

8. Rapidly developing pervous prostration.

9. Rapid breathing.

10. Expired air cold, 
11. Nostrils dilated.

12. Mucous membranes cyanotic.

13. Pulse rapid, small, weak and hard.

In a mild case that recovers the fermentation will stop. In fatal cases the horse most commonly dies from syncope-a result of the great nervous prostration, which is produced largely by the pressure upon the diaphragm, and the animal finally dies from collapse. Death occurs usually from 10-30 hours. The next most common cause of death is asphyxia. The tympanitis is so great and the gas cannot escape because of the cardiac fold of the stomach or of the pyloric end folding upon the duodenum. In such a case the pressure upon the diaphragm is so severe that it interferes with the action of the heart and lungs. The animal may die in 20 minutes, usually, however, in one or two hours. The third cause of death may be rupture of the stomach, which may take place in 4-5 hours. If the case runs on for 3-4 days, and the horse does not die from any of the causes just mentioned, congestion and inflammation may result and the case turn into one of gastritis. Death from gastritis can occur in 1-6 days.

Great flatulence produces anæmia of the walls of the stomach and prostration of the vaso-motor system, and as the flatulence subsides, the atonic blood vessels fill with blood and become congested. Then inflammation follows.

14. The appetite is suspended.

15. Temperature not so much affected until gastritis sets in. Then there will be an elevation of temperature.

Treatment.-This must be directed toward the arrest of the fermentation, which is always present. The cases are always urgent. Give drugs that will arrest the fermentation without producing more gas. The acid to be combated is acetic acid, not hydrochloric. Neutral salts are indicated,- -hyposulphite of soda-the sulphate is most popular and very effectual. Others are sulphite of lime, magnesium sulphate. Such antiseptics as turpentine, boracic acid, salicylic acid, cresylic acid, coal tar emulsion, etc., are good. Carbolic acid also. 

The contra-indications are these:

1. The antacids.

2. Carbonates and bicarbonates.

If the carbonates are used in this condition, more gas is produced by the chemical action of the combination of the drugs in the stomach, and therefore those who recommend them are wrong. On the other hand, the neutral salts tend to liquefy the gas already there.

In every bad case of gastric flatulence it is a good plan to pass a stomach tube. This opens the cardiac orifice and gives relief. The antiseptics can be pumped through the tube and then syphoned out again-this empties the stomach. After emptying the stomach, pump into it a couple of gallons of water with a little common salt dissolved in it, then syphon it out and repeat if necessary. Turpentine is usually given in capsules, may be given with oil. If the mouth is dry, the pure turpentine will scald it. When mixing the turpentine with oil, use equal parts. Bear in mind the nervous prostration and give a stimulant, such as sulphuric ether, alcohol, capsicum, ginger, strychnine, etc. In order to relieve the pain give an ánodinefluid extract cannabis indica, chloral hydrate, and sulphuric ether. Do not give opium, but a hypodermic of morphine, 4-5 grains may be given.

When the horse is relieved, feed on very light sloppy food to avoid gastritis, for this is usually fatal in the horse. Find out the cause if possible, make him eat slower, file his teeth, give feeding of hay before the oats.

\section{ACUTE GASTRIC IMPACTION.}

This condition is an impaction of the stomach, producing stupor; it is ordinarily known as stomach staggers. In such a case the horse gets loose in the night and gets at the grain or bags of flour and loads his stomach with solid food. When the stomach becomes filled in this way, it becomes paralyzed. The horse is in a stupor, he hangs his head, doops his ears, partly closes his eyes, stands with his face against the wall, and inclines his body forward. If the condition comes on when the 
horse is out doors, he will probably stagger along. This usually lasts several hours, or until fermentation of the mass in the stomach takes place, with evolution of gas. Then the animal wakes, bloats, and unless he gets prompt relief, will die. It then turns into gastric flatulence.

Treatment.-The doctor will probably be called during the stage of stupor, as that is the main evidence to the owner that his animal is sick. Bear in mind that the horse is loaded with grain sufficient to kill him. Drench him carefully with quantities of saturated solution of sodium sulphate, magnesium sulphate, or good liberal doses of turpentine. Give two ounces of turpentine every hour, until you have given two or three doses. A little renal congestion may follow so much turpentine, or polyuria, and possibly strangury, but these symptoms are harmless. The purpose of this treatment is to prevent fermentation and to dilute the contents of the stomach. After the danger is passed, give the horse a dose of aloes in solution. A case of this kind is always serious and sometimes fatal. Prevent if possible fermentation. Give liberal doses of antiferments, and then produce purgation. If this line of treatment fails to cure, use the stomach tube as directed for gastric flatulence.

\section{CHRONIC GASTRIC INDIGESTION.}

This corresponds to dyspepsia in the human. It is fundamentally a derangement of the stomach in which digestion is imperfectly performed. It is not dangerous nor even serious but it takes a long time to cure.

Etiology.-Chronic gastric indigestion is usually caused by:

1. Errors in diet.

2. Faulty gastric secretions.

3. Abnormalities affecting the movements of the stomach.

The errors in diet include irregular feeding, too long continuation of one kind of food, bad quality of food, like musty oats, over-ripe hay or moldy grain.

Faulty secretions are usually seen in case of deficiency in se- 


cretion of the hyrochloric acid, or a deficiency of the gastric juice, in which case the quantity of pepsin will be diminished also.

The abnormalities are the chronic thickening of a portion or portions of the stomach wall; chronic thickening of the pylorus, or chronic dilatation of the cardiac orifice of the œsophagus.

Semeiology.-The symptoms consist of the following:

1. Capricious appetite-animal sometimes very hungry and at other times will not eat at all.

2. Horse inclined to lick the wall for the lime and other alkalies; will eat dirt, especially clay, sometimes eats the bedding that has been urinated upon in preference to good hay. Young stallions will sometimes defecate and turn around and eat it.

3. Occasional mild attacks of gastric flatulence, the only evidence of which are sour eructations.

4. Excessive thirst-quite common, animal will drink a great deal of water will try to empty the trough.

5. Polyuria, a result of so much drinking. Often this is the symptom noticed by the owner which induces him to call the doctor. When he arrives, he is told that the horse is flooding the barn. The urine is clear, and there is much of it.

6. Unthriftiness, staring coat, thinness of flesh, pot belly, debility, etc. Animal may become hide bound.

7. Feces usually dry.

8. Pulse, temperature and respirations unchanged.

Treatment.-Give the animal a complete change if food if possible-grass in season, and change the hay from timothy to prairie. If this is not possible, use any mixed hay containing 
timothy, clover and red-top. Early cut timothy will do. Crush the grain for a change and add at least 25 per cent bran to the mass. Salt the feed with common salt.

Give antacids and an occasional laxative such as a pint of raw linseed oil. The best antacid is bicarbonate of soda. For the digestive apparatus prescribe arsenic, gentian, nux vomica, ginger, charcoal, etc. The antacids have a peculiar physiological influence upon the hydrochloric acid secretion of the stomach glands.

If the flatulence occurs within two hours after eating, there is a de'iciency of the hydrochloric acid, and in this case give the hydrochloric acid instead of the soda, or alternate the two. Do not give the two together. Alternate them for a week at a time. A dose of strong hydrochloric acid is about 7 drops of the pure acid (freely diluted). Make up a pint of water and a dram of the acid-add any other drug that you wish.

If the flatulence occurs about five hours after eating, that is indicative of an excess of hydrochloric acid. In such a case give anticids four hours after eating. This will intercept the secretions of gastric juice and limit the quantity, or at any rate will prevent any injurious effects from the excess. If the horse has a fair appetite and will eat powders, give him ordinary white arsenic in his feed along with the anticid; but if his appetite is poor, in addition to the mixture of the tinctures give him Fowler's solution. Calculate to give him arsenic three times a day and it should always be given after eating. Do not allow the arsenic to go into an empty stomach. Never put a strong solution into the mouth in concentrated form-small doses will produce poisonous results. Do not give Fowler's solution clear - it is too strong; it will arrest the appetite and loosen the bowels. Dilute it freely.

In cases of this kind you will have to make many changes in the hygiene usually. You will. find, for instance, that young stallions, between one and two years old, and older ones if they belong to the draft class, suffer from confinement. You will frequently find over acute, subacute or chronic indigestion in stallions, especially in young ones. Often the main symptom noticed by the owner is the refusal of the animal to eat, and this 


induces the owner to call the doctor. For this lack of appetite give a mild purgative and follow it with antacids. Let the horse fast for two or three days.

In older horses with this trouble always examine the teeth. You will find that deranged teeth are often the cause of indigestion.

In case you have a young stallion to treat for indigestion, always prescribe exercise, the lack of which is one of the causes of his trouble.

\section{GASTRITIS.}

Gastritis is inflammation of the stomach mucous membrane. This disease goes through the same course as any other inflammation. It frequently runs to the third stage of inflammation and may terminate in ulceration. It is not very common in horses but is common in dogs and cats.

Etiology.-In the horse gastritis usually. follows a long continued course of gastric flatulence. It is sometimes caused by traumatism, by foreign bodies-by anything that can wound the mucous membrane: In this case infection often follows. The gastric mucous membrane is frequently involved in a general inflammation along with a similar condition in the whole alimentary track, as seen in influenza. Poisons, such as caustic potash, any of the three vitriols (blue, white and green), arsenic (common form eaten is Paris green), etc., are some of the causes of gastritis. Others are mercury, lead, and vegetable poisons, such as the yew, rhododendron, etc.

Semeiology.-The symptoms consist of colicky pains, not unlike those of colic. The animal turns his head around to one side, has a depressed and haggard appearance; he sweats more or less; respirations quickened; pulse rapid, hard and small, and gets more so as the disease progresses. There is complete anorexia. In the very acute cases the thirst is not increased, but in subacute cases the thirst is greatly increased. The saliva is usually ropy. There may be frequent eructations; flanks tucked up; purgations; constipation. The animal dies in a stupor, and the form of death is syncope. 
In the dog the symptoms are as follows: vomiting-a dog will drink large quantities of water and will go right off and throw it up again, then come back and drink some more. This is one of the most prominent symptoms of gastritis in the early stages. Upon pressing on the stomach, pain is produced; the dog lies flat on his chest. The other symptoms are much the same as in the horse.

Post mortem.-The mucous membrane of the stomach will show inflammation. If caused by long continued gastric flatulence, the villus lining (near the pylorus) will be dark red and swollen. If the inflammation is caused by caustic poisons, there will be deep erosions. In some cases you will be puzzled to account for the death, the lesions not seeming sufficient. In case of lead poisoning the stomach at post should show redness without erosion-this poison produces general collapse. If the stomach has arsenic in it the mucous membranes will be dark.

If death from poison is sudden, there will be no change in the fecal matter, i. e. in arsenic poisoning; but if the case is one of 24 hours standing, the fecal matter will be black; if 3-4 days, there will be black diarrhœa.

Treatment.-First ascertain the cause. If gastritis occurs from poisons give the proper antidote. If it occurs from a caustic, give oil-the oil and the alkali will form soap. Give moderate doses of the oil and then repeat. A pint of the oil would be sufficient. Repeat in half pint doses three times a day. To a large horse give a pint at the time. Apply a mustard plaster over the belly. Give aconite, alcohol and an anodyne such as fluid extract canabis indica. Give a bland fluid to drink like linseed tea. If the thirst is intense, restrict the water supply-put a little bicarbonate of soda in the drinking water. As a special regulator give subnitrate of bismuth, combining it with salol. During convalescence give lime water, fluid extract gentian, strychnine.

As regards the poisons which case gastritis, it is essential for a doctor to keep posted on the various antidotes. It is a good plan to carry a dose book in one's pocket all the time. 



\section{CHRONIC GASTRITIS.}

Horses are affected occasionally with chronic gastritis. It does not occur often, but when it does, it does not yield to treatment readily. It is associated with the following conditions:

1. Continual errors in diet.

2. Frequent recurring fits of acute indigestion.

3. Textural changes in the glandular tissues of the stomach wall, such as seen occasionally in heaves. The secretions are deficient in both quantity and quality.

4. Cribbing and long-continued wind sucking.

5. Diseases of the liver interfering with the secretion of the bile.

6. Malignant disease of the stomach.

7. Mechanical injury to the mucous membrane produced by bots. These hang to the mucous membrane with little hooklets, and if there are very many, they irritate the mucous lining.

Semeiology.-The symptoms are long continued and not very expressive. A very capricious appetite will be present; greatly increased thirst; general unthrifty condition; pot belly, hide bound, long staring coat, debility, etc. The termination may be favorable if the cause can be removed, but it may develop into the acute form at any time.

Treatment.-Look at the molar teeth and attend to them if need be. Attend to the hygiene; give antacids such as bicarbonate of soda, lime water, prepared chalk, subnitrate of bismuth, etc. Put them in the drinking water or feed. A horse with this trouble will drink all that he can hold. Other drugs are gentian, ginger, mux vomica, tonic doses of quinine, arsenic, etc. Give him a run at grass if nossible. See the Hygiene for Chronic Gastric Indigestion. 


\section{RUPTURE OF THE STOMACH.}

Rupture of the stomach may be partial or complete. Remember that the stomach wall consists of three coats, the middle layer of non-striated muscle, covered with a serous coat, the peritoneum, and an inner mucous coat which lines the stomach. In partial rupture the serous coat gives away first, then the muscular.

Etiology.-Rupture may occur in gastric flatulence, repeated attacks of which will terminate in rupture. The rupture will probably occur in a much milder attack of flatulence than he may have recovered from many times before. Every attack of flatulence weakens the stomach wall. In posting a case of rupture, the tear may be a foot long-this probably took place when the horse fell. That which occurs in case of distension is usually 2-4 inches long. And the post will reveal something about the time of the rupture; if it has taken place $6-8$ hours before death, the edges of the laceration will show congestion or even inflammation; if the tear took place when the animal fell, the edges will not be congested.

Semeiology.-The symptoms of rupture are not diagnostic. Never state the diagnosis positively, to be contradicted afterward by a post. The following symptoms are usually confirmed by post mortem in case of rupture but they are present in other diseases too:

1. Vomition is a usual symptom. There is not necessarily an actual expulsion of food and injecta, but there may be retching. In some cases there will be no vomiting at all. In rupture of the diaphragm and in gut-tie we get vomition also. Horses have vomited 2-10 hours after rupture as proven by the congestion and in some cases inflammation of the edges of the rupture. We may find an extensive peritonitis in case of rupture, this showing that time may elapse and the ani- 

mal live after the rupture has taken place. The peritoneum may show exudation and effusion as the result of the contact with the injecta from the stomach.

2. Great nervous prostration.

3. Increase in frequency and decrease in size and strength of the pulse.

4. Sweating in patches.

5. Anxious countenance.

6. Animal lies down and then rises up on his forward feet, sitting on his haunches like a dog.

7. Hurried respiration.

8. Mucous membranes cyanotic-the cyanosis is due to the nervous prostration, which lessens the heart's action and thus induces the cyanosis.

As death approaches, the ears, extremities and nose get cold; the perspiration is cold; there is trembling of the muscles, more particularly the caput muscles. Death may take place anywhere from $1 / 2-20$ hours. In the majority of cases it occurs in 2-6 hours. The nervous prostration and death from syncope may occur at any time. A sensitive nervous system will collapse quickly from an attack of. rupture and the animal will die in half an hour, while a phlegmatic temperament will not show so quick a reaction. One case is recorded as living 50 hours after rupture.

The symptoms after the rupture has taken place are as follows: .

1. Pain ceases.

2. Tympanitis ceases.

3. Sudden cessation of the flatulence.

In a case where rupture occurs say 18 inches long without any congestion of the lacerated edges, we must conclude that the rupture took place when he fell, and that he died from asphyxia. In such a case you could not say that the rupture killed him, it was the dropping. On the other hand if you find a partial 
rupture and the mucosa bulging outside the wall, then the rupture causes the death.

Treatment.-To begin with all cases of rupture are fatalwhether the rupture is partial or complete. But seeing that the symptoms of rupture are not very diagnostic, the doctor must continue the case right up to the end. Treat the case for acute gastric indigestion.

Occasionally at post mortem, holes will be found in the wall of the stomach, varying from the size of a dime to a dollar. A horse dying in the city which recently has come in from the country will always have bots. To these some attribute the cause of the holes, and it is also common to refer the cause of colic to bots-men say that the horse has the "bots." This is wrong; the bots are not the cause of colic, or of the perforated condition of the stomach as found post mortem. The bots may cause gastritis by irritating the stomach mucosa or they may become so numerous as to make an obstruction when they let go, the only two conditions in which they can be responsible for any lesion. The real cause of the holes in the stomach is this: They occur in a digested condition of the wall. In such a case the animal has probably been in a clover patch where in half an hour he can eat enough clover to blow himself up like a balloon! He dies, and if the post mortem is delayed 4 or 5 hours, the stomach will become digested in spots and patches, and warm weather will aggravate the condition. The abundance of the gastric juice which was present at the time of death digests the stomach wall in patches. This is known as post mortem digestion.

\section{CONSTIPATION.}

Definition.-Constipation is a condition of the bowels in which the feces are unnaturally retained, or if ejected, are scanty, hard and dry. It is not usually a serious matter, but it occasionally leads to death through other developments. The large intestine is the part involved; constipation usually occurs in the floating colon or rectum.

Etiology.-The causal factors are three: 

1. Feeding on bulky, innutritious food with too little water.

2. Defective peristalsis, amounting in some cases to paralysis of the large bowel.

3. Deficient secretion of the succus entericus or excessive absorption of the fluid portion of the food.

\section{Intestinal obstructions.}

Pathogenesis.-In regard to the first cause, bulky food will not cause constipation if the horse gets plenty of water; deficiency of water, however, will of itself cause constipation whether the food is bulky or not. But suppose the horses are wintering in the barnyard on straw, and have to go a long way to get water. If the weather is cold, they will not go so often, and furthermore they will not drink much when they do go. This causes constipation. Or it may be mid-summer when the grass is dry and short. The animals have to go into the bottoms to get the grass and the streams are dried up. They get bulky, innutritious, dry hay and little or no water. These are two conditions to cause constipation.

In cases of constipation from the second cause, we shall find that the horses are over-fed, perhaps kept for a long time on one kind of feed. As a result the secretions are defective, the nervous system is tired, and this fatigue reacts causing paralysis of the bowels. The third cause, a deficient secretion of the succus eritericus, is a peculiar condition, and may be due to inactivity of the glands, or to excessive absorption of the fluids of the bowel. The features peculiar to the fourth cause will be given under the head of Intestinal Obstructions.

Semeiology.-The symptoms of constipation depend upon the cause. In case the first cause is operating to produce the disease, there will be a general unthrifty condition. The horse will be thin, more or less hide-bound, his coat will be long and staring and the feces scanty and dry. If the constipation is caused by the second factor, we shall get inactivity of the bowels due to nervous depression, which, in many cases, means paralysis of the colon. In the morning you may find an absence of fecal mat- 
ter, the horse perhaps will not eat his breakfast, and is inclined to lie down a good deal. If you drive him out, he will paw, and if loose in the box stall he will turn around and lie down again. This condition continues, gradually growing worse in the way of pawing more, getting up and down at shorter intervals, switching the tail, etc. At the end of the third day or the beginning of the third, the temperature rises. It may be $102^{\circ}$, and the pulse is increased in frequency about 50 . By the end of the third day the temperature will be $103^{\circ}$, pulse 55 ; fourth day, temperature $105^{\circ}$, pulse 60 and getting small and hard. By the morning of the fifth day the animal is in bad condition,-temperature $105^{\circ}$ or more, pulse 60 to 70 , respirations 25 or 30 , haggard countenance, injected mucous membrane, etc. He begins to tramp,not get relief before night, he will die from enteritis.

If the constipation is caused by a deficient water supply, the rectum is apt to be everted to the extent of two or three inches. This is caused by straining. The everted rectum is very dark red, and if it stays out very long it becomes nearly purple.

If the third cause is the factor producing the condition, the pellets will be small, hard and dry, sometimes falling on to the floor like marbles. In aggravated cases of this kind there will be an inclination to lie down more than usual and mild colicy pains when standing. This form of constipation occurs in driving horses that are highly fed on hay and oats and are overworked, keeping them thin in flesh.

In constipation from the first and third causes 5-15 days may elapse before enteritis develops, but in all cases, if the horse is not relieved, the enteritis will eventually kill him.

When a foal cannot have its first passage on account of the dryness of the meconium, you will find that the mare has been worked too hard and kept on dry feed so that there is not enough water in her system and the young foal partook of the same dryness from which the mother suffered. If the mother is kept on soft feed for a month before the birth, this is not likely to occur. In case the foal does not have a passage by the end of 10-12 hours, he must be relieved.

If the constipation is due to the second cause, the contents of 


$$
\text { . }
$$


the bowel in this case are not hard and dry; there is no impaction nor disturbance, simply paralysis of the bowel. The normal motion of the fecal matter has ceased, and the contents are lying persistently in one place. This acts as an irritant but not enough to stimulate the bowel to functional activity, enough, however, to produce congestion and eventually inflammation. The paralysis of the bowel is probably caused by too long continuation of one kind of food; the bowel gets tired, and this tired feeling may be due to a loss of irritability in the peripheral terminations of the sympathetic nerves, which supply the bowel with motor power.

Treatment.-Naturally constipation would indicate the use of a purgative, but much judgment needs to be used in treating a case of this kind. As a rule, the drastic purgatives are contraindicated. The oleaginous, lubricating purgatives are better. Consequently we give raw linseed oil instead of aloes, sulphate of magnesia, or sulphate of soda. Sometimes these saline laxatives work fairly well when the case is due to the second cause, but otherwise they are not useful in equine practice. The linseed oil stimulates the bowel sufficiently and it also lubricates it. It can be repeated with safety, but not so with the other purgatives. In cases of severe constipation, aloes will not work; if you repeat the dose even after a reasonable length of time, and the bowels do finally get started, super-purgation and death will probably follow. In addition to the oil, the horse needs a stimulant, which in this case should be strychnine. I think that strychnine in constipation is indispensable; it will do more to cure the trouble than any other drug. You can use other stimulants right along too, such as ginger, capsicum, and carbonate of ammonia. Further, give enemata (soap and water); these are indispensable, too. They should be warm, about 100 degrees.

As a rule, in treating constipation, anodynes are not necessary until the case gets along into a late stage, when the pain may be so severe as to require an anodyne on humane principles. We are inclined to think that they tend to decrease peristalsis, which you want to increase, and therefore they must be such as will not interfere with the very thing you are trying to bring about. Chloral hydrate and chloroform can be given if necessary. 
To an average sized horse we usually give a quart of linseed oil. Combine with this an ounce of fluid extract of nux vomica and a dram of the fluid extract of ginger, or half a dram of fluid extract of capsicum. In giving a soap and water rectal injection, do this by gravitation. You can use a pump, but gravitation is better. Hang a bucket on the wall and let the solution run in through a hose, which should be inserted in the rectum anywhere from 2-6 feet. In this way more can be introduced than with the pump. The pump forces the water in, but the bowel resists it and will throw it out quicker. Consequently the water does not stay in long enough to soften the fecal matter. The injection should be repeated every 3-4 hours. The oil should be repeated night and morning in pint doses, but with each repetition, lessen the quantity of nux vomica. Give an ounce the first dose and half an ounce every subsequent dose."

Suppose the trouble runs on and does not yield to this treatment, give stronger remedies, such as eserine, arecolin and barium chloride. You can give a grain and a half of eserine with a half grain of strychnine dissolved in a dram of watergive hypodermically. Fifteen or twenty minutes before giving these doses, give half an ounce of cannabis indica. Or give him morphine, but cannabis is better than morphine. If this does not work, in the course of two hours give a grain of arecolin and a half grain of strychnine. Dissolve in water and give hypodermically. Some add to either or both of these from $i / 4$ to $1 \frac{1}{4}$ grains of pilocarpine.

If the horse gets no relief by the fourth day, and the fever begins, the pulse begins to climb up, his countenance is distressed, etc., the doctor begins to get worried. If warm enemas have been given, change them to cold-this will help very much. Run the hose in slowly five or six feet and use plenty of water. The cold water will reduce the temperature, which by this time may be 106 degrees. In half an hour the temperature will come down perhaps two degrees. It will allay the pain and stimulate the sympathetic system. This cold injection often saves a horse. This is especially true if the case does not yield to eserine or to arecolin.

Never repeat eserine or arecolin oftener than three hours 

apart. If you repeat them alternate them, and do not forget that the stimulants are as useful as any other part of the treatment. In case of an infant suffering from constipation, give a soap and water injection, inserting the finger, and let him have an ounce of New Orleans molasses, or a half ounce of linseed oil, or an ounce of castor oil.

If in 6-10 hours very urgent symptoms come on, do not wait for the action of oil, but resort to eserine or arecolin. Dr. Quitman cuts out the eserine in case of complete paralysis of the bowel, but if the strychnine is given first to stimulate, then the eserine will work all right in most cases. Eserine should not be repeated under 3 hours. The dose is $1 \frac{1}{2} 2$ grains.

Horses afflicted with any kind of paralysis in any part of the body will take large doses of strychnine.

Counter irritation is good in cases of constipation. If mustard does not seem strong enough, then take croton oil. Mix croton oil (1 part) with linseed oil ( 8 parts). Thะ doctor should apply this externally himself, and be careful not to blemish. The irritant acts as a purgative as the result of absorption.

In obstinate constipation we hind that massage of the bowels is very practical, especially for dogs, cats and the humar. It cannot be applied to the horse. In treating the dog, lay him on the left side, fill him up with soap and water and firmly work the water out of the colon. Begin on the right side and work along the colon and then on the left.

\section{DIARRHOEA.}

Definition.-Diarrhœa is an unnaturally fluid condition of the feces. It is a peculiar functional disturbance in which there is an excessive secretion of the succus entericus with increased peristalsis. This additional intestinal juice helps to produce the fluidity of the feces. Accompanying the diarrhoea are increased peristalsis and increased amount of mucous and gelatinous exudate accumulating on the mucous membrane of the bowel. This is a catarrhal condition, 
In superpurgation we have diarrhœa, but it is not functional; it is the result of the action of the dose.

Etiology.-Diarrhœa is caused by the action of some irritant in the bowels. This irritant acts as a stimulant. Undue fermentation may be present, brought about by specific germs, especially fungi and molds. Or there may be inactivity of the liver in which there is a defective secretion of bile,- - there is not bile enough to change the acid chyme to the alkaline chyle. If the contents of the bowel are not alkaline, then diarrhœa takes place. This is the cause of 95 per cent of all the cases that occur. Parasites, various intestinal worms, especially the ascaris megalocephala, tape worms, mechanical and chemical irritants, local irritants such as would produce a local inflammation with alteration of structure, excess of bile which is of itself a laxative (ox gall was the old standard family laxative), bad hygiene (poor food, such as hay cut from the bottoms. which overflowed the previous spring, the action of the sand that sticks to the grass and is eaten with the hay being the cause of the diarrhœa; foul water containing wigglers and parasites, and irregular and overfeeding), exposure to cold and dampness, overheating especially on a hot day and some forms of indigestion-these are the principal factors producing diarrhœa. Diarrhœa is also a symptom of influenza.

Semeiology.-In diarrhœa there is usually a watery discharge from the bowels, often spoken of as alvine discharges. The color is dirty brown or it may be clay or yellow. The dirty brown is the usual color when the liver is not affected. The lighter color indicates a deficiency of bile, a yellow color an excess of bile. In case of an excessive secretion of bile there will be a switching of the tail prompted by the irritating action of the bile, which scalds the anus. This is bilious diarrhœa.

In all cases of diarrhœa evacuations are frequent; in mild cases the discharge may be frequent too, but it is not so fluid. There is more or less pain present, and in bad cases actual cramps. If the case is acute the animal may lose a day or two from his work. Increased secretion and increased peristalsis are nature's remedies to get rid of irritants. Consequently in diarrhœa we find the mouth pasty, like the coated tongue in the 

human. The mouth smells sour. As a result there will be a lowering of temperature, i. e., subnormal. The amount of the fall of temperature depends upon the nervous prostration which results from the evacuations. The mucous membranes become blanched and they become paler as death approaches; the pulse gets small, weak and hard; animal has a dejected appearance; he sometimes sweats in patches, as death approaches; ultimately he dies from syncope, or collapse.

Diarrhœa runs a very rapid course; in young animals 6-10 hours may be fatal. In case of superpurgation (an overdose or injudicious repetition) the course is very rapid. Do not repeat a dose of aloes under seven days, for you are liable to get serious results. Be very careful about repeating purgative doses. Some say that oil will kill a horse. "I know better." You can give him a gallon in some cases when needed. It is better to let the horse die from other trouble than from superpurgation.

The bowels of the horse are very sensitive; I do not believe in severe purgation for it is weakening. The danger lies in injudicial repetition of the dose.

The strongylus tetracanthus sometimes sets up an enteritis rather than a diarrhœa.

In all cases where the temperature runs down, the surface gets cold, especially in young animals. Foals shut up from their mothers should not be separated for more than four hours, for the milk will spoil and produce diarrhœa in the foal. Let the colt suck some every four hours. Never overheat a mare suckling a colt; for this produces an unthrifty condition of the colt.

Post mortem.-General pallor; the mucous lining of the bowels is pale; intestinal epithelium eroded in places over extensive areas; may find inflammation; peritoneal coat spotted; ecchymosis; contents of the rectum more or less bloody, called by some dysentery, but it is really bloody diarrhœa. In dysentery we have a rise of temperature; in diarrhœa, a lowering.

Treatment.-Remove the cause. For the excessive peristalsis opium is the only remedy; for the acid condition of the bowels give antacids. If the fermentation is microbic, give antiseptics. Salol is the best; it is effectual and harmless. Opium 
is best given in powder by the mouth. To prevent nervous prostration, give a stimulant-zingiber or capsicum, or if the case is serious, ammonium carbonate or alcohol. Strychnine is contraindicated. The object is to tide nature over while the other drugs get to work. In acute cases astringents are useless; in chronic they are indispensable. In cases of bloody feces, give an injection of starch gruel with laudanum. When the bowels are running it is unsafe to stop them too quickly for you are apt to get enteritis. For dogs, cats and people give subnitrate bismuth (10 grains) and salol (5 grains) every four hours.

\section{ACUTE DIARRHOEA IN THE HUMAN.}

Treatment.-This consists of antacids and antiseptics, the antacids to neutralize the contents of the stomach and bowels and the antiseptics to arrest the fermentation in them.

Prescription-

Salol .............. 5 grains

Bismuth ..............10 grains

Take every four hours.

Both these drugs are harmless and two or three doses usually produce the desired result. This prescription is good for man as well as for animals. In grown people diarrhœa does not always yield to the antacids and it is necessary to change to acids, in which case use sulphuric acid.

Usually excessive thirst is a symptom of diarrhœa; sulphuric acid cures this and stimulates healthy secretions when the antacids fail.

\section{CHRONIC DIARRHOEA.}

Chronic diarrhœa is exceedingly disagreeable. The horse comes out of the barn in a normal condition so far as the bowels are concerned, drives a mile or two, when he begins to empty himself and keeps on getting looser as he travels. This is particularly true of driving horses.

The cause of this trouble lies in over-heating the horse when 


\section{-}



he is in a plethoric condition. This purges him and he never gets over it. The mucous membranes become excessively irritated and this excites the sympathetic nerves which control the bowel. The result is chronic looseness. Chronic diarrhœa can be controlled to a certain extent but it cannot be cured.

Treatment.-Give antacids and astringents. Hygiene will do more for the animal than any other measure. Give the best quality of food in rather limited quantities, especially hay. The army allowance for a horse is fourteen pounds a day, this for an average sized horse; in this condition give 10-12 pounds. Add dry bran to the oats to make him chew slowly and he will masticate better. If he is inclined to drink too much water measure the water for him; give it to him in a pail, $1 \frac{1}{2}$ gallons four times a day, six hours apart. If the thirst is excessive put a little bicarbonate of soda or hydrochloric acid in the water. Do not give any corn. Keep him on oats and bran.

$\mathrm{R}$

Prescription

Sodium Bicarbonate.............. 4 ounces

Gentian Rd. Pulv................. 3 ounces

Quercus Rd. Alba Pulv............ 16 ounces

Zingiber ................... 1 ounce

Charcoal ................... 3 ounces

Make into 60 powders and give one night and morning.

Recommend the driver of the horse to start out slowly, and go that way for an hour, increasing the pace of the animal gradually. Especially is this necessary in hot weather. In bad cases it is necessary to give this treatment continually, and the horse will keep in a very comfortable condition. After a month's treatment leave it off for a week or two then repeat periodically.

The main treatment is the hygiene. Use soft diet such as scalded oats for horses. If you give ground feed a little bran should be added; pour hot water on it and steam well, adding a little salt. For horses that scour from indigestion stir up a little wheat flour and cold water (a pound of flour to a gallon of water) and give that. This will often be effectual.

The following is the classification and description of the three principal worms associated with colic:

1. Ascaris megalocephala, inhabiting small intestines. 
2. Strongylus armatus (sclerostoma equinum), found in the large intestines of the horse.

3. Oxyuris curvula (commonly called pin worms), found in the intestines and rectum of the horse.

The ascaris megalocephala belongs to the family ascarides of the order of nematodes. It is the largest species of its kind. It is a large, round, yellowish white worm from 10-14 inches long. It is oviparous and common in the small intestine of the horse, ass and mule. When these worms are numerous enough to cause an obstruction they may cause colic.

The strongylus armatus is a blood sucking worm found in the large intestine of the horse. It is from 1 to 2 inches long; body gray or reddish brown and broad in its anterior part. The moutii is provided with a ring of fine teeth by which it holds to the mucous membrane. The larvæ of the worm may be found i1i aneurisms and may produce tumors as large as a hazel nut in the wall of the intestine (verminous cyst).

The oxyuris curvula is the ordinary pin worm and is most common in the rectum. It measures from 1 to 3 inches long; curved in the anterior part of the body; the posterior half is attenuated. Its mouth presents three great rounded lips. It belongs to the family oxyuridæ of the order of nematodes.

Ascaridae (family).

Strongylidae (family).

[Ascaris megalocephala (species).

Large round worm (common).

「Strongylus armatus (sclerostoma

The armed worm.

Oxyuridae (family). equinum).

$\left[\begin{array}{l}\text { Oxyuris curvula (species). } \\ \text { Common pin worm. }\end{array}\right.$ 



\section{COLIC.}

Colic in the abstract, of course, relates to the colon; but as a disease we apply the word to peculiar abdominal pain. Colic is divided into two classes, spasmodic and flatulent.

Spasmodic colic is a painful affection of the bowels without fever or inflammation. The pain is intermittent and is produced by irritation of the mucous lining of the bowels. This irritation extends to the muscular coat, which contracts spasmodically through the stimulation of the sympathetic nerves. This contraction is usually violent and painful. It is the effort of nature to increase peristalsis to push the irritant along. If nature is successful in doing this, the trouble ends; but if not; then the action is repeated. Therefore the pain is intermittent. If nature fails entirely, the irritation will later produce congestion and inflammation, and eventually death by enteritis.

In such a case the spasms increase in violence as the case runs along. In a favorable case they may stop aitogether, but if they do recur, it is with decreased violence until they stop.

Etiology.-As to the causes of colic, first comes errors in diet, producing indigestion. This covers overeating, poor food, heavy drinking soon after eating, frozen food,-this chills the mucous lining and the reaction from the chill is accompanied by cranips, eating when physically exhausted, drinking large draughts of cold water especially when warm, and still more so if the horse is tired, intestinal concretions,- -usually the calcareous ones (a calculus in the bowel does not cause pain until it rolls out of its pocket, then it acts as an irritant, but the pocket in which it grows has become accustomed to its weight gradually), parasites such as ascarides, strongylus armatus and strongylus tetracanthus. Of these three worms the last two mentioned are more likely to produce colic, the first to produce diarrhœea. 'The young embryos of these worms sometimes work their way into the colic artery, start a colony and eventually cause an aneurism, which is more or less obstructed by worms. Any alteration or disturbance of the circulation in the bowels produces violent pain the same as congestion in pleurisy causes pain. This pressure in the colic artery produces pain sufficiently to ultimately kill the animal. 
The strongylus tetracanthus usually exists in large numbers and produces extreme pain, for which we use irritating poisons such as arsenic, sulphate of copper, sulphate of zinc, or sulphate of iron in large quantities.

Another cause of colic is exposure to cold and dampness. If a horse falls down or lies down on the ice or snow, exposure to the cold is likely to produce colic. Colic may be produced by violence in the form of kicks, severe accidents, etc.

Semeiology.-Colic usually comes on suddenly. The irritant, whatever it is, irritates the lining of the bowel and nature will submit to this disturbance only up to a certain point. Then she gives away suddenly. The irritation sufficient to produce colic may be accumulating for several days or it may do its work in a few hours, but when the colic begins, it runs a rapid course. If the horse is watched prior to the development of the disease, it will be noticed that he gets uneasy first, may lie down and kick his hind feet up, turn around, switch his tail, lie down and roll and get up again. He may be quite comfortable for a few minutes, then another cramp comes on a little more severe than the preceding one. As a result he lies down and may attempt to remain on his back, then goes through the same restless motions again until he exercises himself enough to sweat. He rolls and kicks and switches his tail until he feels easier.

In the majority of cases nature cures the trouble. These violent contractions succeed in moving the obstruction along. Seventy-five per cent of these cases will be well when the doctor arrives. The pulse is increased in frequency due to the violent exertion, but it is not altered in character until the trouble has been running a considerable length of time with the possible danger of enteritis. The exercise may also cause an elevation of temperature.

Pressure on the belly may give relief, while in enteritis or peritonitis, it increases the pain; this is a means of diagnosis.

According to the idea of the laity retaining the urine causes colic, but this does not occur except when the horse has a habit of urinating under certain favorable conditions and holds the urine too long, thus causing a spasmodic contraction of the neck of the bladder. This is painful in itself and of course he is un- 

able to void his urine. Overdistension of the bladder also causes pain. Some horses refuse to urinate while in harness; others will not urinate on the bare floor.

Most all cases of colic are due to the first cause-errors in diet. The effect of this is indigestion, and we can say that seventy-five'per cent of the colic cases are due to indigestion.

The feces in most cases are hard and dry. If the trouble is in the large bowel, the horse will stand stretched out as though he wanted to micturate. This action on his part is usually looked upon as a symptom of the disturbance being in the large bowel, but the owner gets the idea that the irritation is in the bladder. Again, when the large bowel is involved in colic, the horse will usually resist the entrance of the hand into the rectum, but will not if the trouble is in the small bowel.

Occasionally we find that indigestion produces diarrhœa,quite an extensive loosening of the bowels with severe cramps in connection with it.

Treatment.-Bear in mind that the trouble is always urgent, therefore be quick. If you delay, he will get well without your help! On the other hand he may develop enteritis. Stimulation is the main point as regards curing the horse. The object of this is to assist nature in forcing the irritant, whatever it is, along through the bowels. Of course these stimulants naturally increase the pain, but by so doing you are assisting nature to overcome the trouble. If the contents of the bowels lie along in one place, this will produce inflammation and death. The stimulants usually given are the more active ones such as sulphuric ether, liberal doses of nux vomica, strychnine, ginger, small doses of eserin or arecolin, barium chloride, aromatic spirits of ammonia.

Give anodynes freely, such as chloral hydrate, tincture of aconite (freely dilute it in small doses and repeat often), cannabis and in exceedingly violent cases hypodermic injections of morphine, not desirable but allowable. Friction on the belly gives relief. To overcome the indigestion use some neutral salt to act as an antiseptic. Give sodium theosulphate in 2-ounce doses. Turpentine is an excellent remedy, being an antiseptic and a stimulant. Ginger and capsicum are also good remedies. 
Watch the case closely; stay with it for a time. Colic doses are usually repeated in half hours but sometimes they must be repeated every 15 minutes. For violent colic away from home, at a funeral for instance, tie a small piece of tobacco to the bit, and the horse will swallow enough of it to give him relief.

\section{FLATULENT COLIC.}

Flatulent colic is similar in many respects to spasmodic colic, but instead of the cramp we have tympanitis. This, whether of the bowels or the stomach, is always due to the fermentation of food. In case of impaction of the colon, when there is paralysis of the bowel, there is sometimes a little flatulence, but it is never a distressing symptom. In that case the flatulence as it occurs passes off naturally.

In flatulent colic the tympanitis distends the bowels and presses strongly forward against the diaphragm. This disturbs the breathing, making the horse breathe faster and more shallow, producing rapid nervous prostration or asphyxia. Rupture of the bowels occasionally takes place, the symptoms are selfevident. The body is distended, the flanks tympanic or drumlike, and the rectum sometimes everted more or less.

Pathogenesis.-There is indigestion. The undigested food ferments; the flatulence starting in one portion of the bowel produces a fold on itself so that the gas as it forms, cannot escape and the more gas that forms the greater is the pressure upon the fold. Then it presses forward upon the diaphragm and causes asphyxia, or nervous prostration. Naturally as a result of this, the mucous membrane becomes greatly cyanotic; breathing is more rapid and shallow; the ears droop; the head hangs and in the course of 2-4 hours the horse in many cases is ready to topple over.

Treatment.-In a very bad case, when the doctor arrives, he has not time to wait for medicine to act; you cannot relieve the case with the hose and the disturbance being in the bowels, it takes the medicine a long time to reach that place. In the meantime the animal is likely to die. Consequently you must tap the distended bowel with a trocar. This is considered by some a 


radical operation, but with horses it seems quite necessary. Keep the trocar with you all the time. Keep it well cleaned. Boil it after each operation.

We usually tap on the right side for that is the most prominent point of the distension. Clip the hair off from a small place, scratch it with the finger nail and then wash the part with an antiseptic solution. Make a puncture about 5-8 to 1-2 inch deep and then insert the trocar downward, inward and forward, at least six inches, especially in a fat horse. If you strike the bowel, gas will escape. We find two kinds of gas in these cases, sulphuretted hydrogen and carbon-dioxide. The first is the one usually found. Touch a match to the escaping gas and it will burn with a blue flame, if it is hydrogen gas, but if it is carbondioxide the flame will be put out. If the force is great, it may make a flame a yard long. Just as soon as all the gas has escaped, reinsert the trocar into the cannula and pull the two out together slowly. This will prevent the fecal matter from coming out into the peritoneal cavity. Put some vaseline on the opening to keep the dirt out. If possible keep the horse standing during the operation. Occasionally he will lie down and you cannot get him up. Tap on the right side if possible, but either side will do.

After the gas has escaped the horse can get up. Then dose him. The best remedy we have is turpentine. It is quick to act-it will act in ten minutes. No other drug will act so quickly. In addition to the turpentine it is a good plan to give a liberal dose of theosulphate of sodium-4-6 ounces. After the flatulence has been relieved, give an injection of soap and water. In case of considerable pain, give an anodyne; the best one is probably chloral hydrate. Give a 1200-1b. horse about two ounces, either in a capsule or in tissue paper. Give liberal doses of linseed oil.

Occasionally when tapping a horse a stream of blood will come out through the cannula, but I have never known this to be serious. In case you do not get the gas the first time you insert the trocar, keep on trying until you do strike the bowel, always inserting it in a new place each time. In searching in this way for the bowel do not pull the trocar out through the skin, but to it only, then change the direction a little and push it forcibly 
down again. Sometimes the owner will not let you tap his horse, which may be an especially valuable one. Assure him that the operation is a perfectly safe one-that it is absolutely harmless. If he still refuses, you can resort to eserin or arecolin, which should be given hypodermically. When using these violent remedies, give the animal an anodyne so as to blunt the sensibilities; for these drugs will increase the pain. In a moderately bad case, give the eserin, or arecolin, or barium chloride-one dram in a pint of water by the mouth.

There is little use to give injections while the abdomen is distended. Bear in mind that getting the gas out does not cure the trouble; this does not remove the cause. You must introduce an anti-ferment through the mouth.

In tapping for this trouble there are only two organs to avoid, the bladder and the kidney. If you puncture straight down, you are likely to strike the bladder. Tap downward, inward and forward and you will go in front of the bladder. To avoid the kidney dip downward.

Some unfavorable results occur from tapping. These may be enumerated as follows: Peritonitis; tetanus; abscess; intestinal fistula. Peritonitis and tetanus do not occur very often, tetanus probably the more frequently of the two. The germ producing it is introduced with the trocar. Peritoneal abscess is the most common result. It is located between the parietal peritoneum and the skin. This abscess is very serious if neglected. The pus that forms in it will extend to the flank and break out there. If this occurs, open the abscess and let out the pus, then syringe it out thoroughly, and it will heal very soon. This condition is no reflection upon the doctor, such abscesses will occur in spite of good technique in operating. Exercise all possible precaution against dirt to reduce the danger. Sometimes you will get a mesenteric abscess on the inside. The resisting power of horses is strong except in the peritoneum. In case of intestinal fistula, there is a chronic discharge from the bowel through the puncture. The liquid portion of the bowel contents comes out through the puncture and it does not take long for this to become a fistula. The best treatment is to cauterize it. Inject into the opening a solution of sulphate of copper the strength of an ounce to a pint 

of water-that is a little less than 8 per cent solution. Shoot down through the fistula about two drams of the solution and smear the external opening with vaseline. This injection will produce a sloughing and inflammation and the debris will empty into the bowel. Usually one injection will cure the trouble in the course of a week.

In using the trocar and canula, do not leave the canula in the tissues more than two or three minutes. Congestion may gather around it and fix the tissues so that when you take it out an opening remains. Two or three minutes is long enough.

In all bad cases there is great nervous prostration. For this give alcohol, strychnine, ginger, capsicum, and oil of turpentine.

In close ribbed horses you will find that the flank does not distend much and is not so drum like as in a long flanked horse. These cases require more prompt attention than long flankeri horses. Watch the mucous membranes for cyanosis indicating the necessity for tapping.

\section{INTESTINAL OBSTRUCTIONS.}

The intestinal obstructions consist-of calculi, dust balls, bots or any other foreign object that might get into the bowels. As applied to horses these will probably cover all that you will ever find. Milch cows are inclined to eat all kinds of stuff, such as table cloths, etc., dogs are inclined to swallow marbles, spools of thread, and in fact any hard substance.

Other obstructions of the intestines are caused by:

2. Hernia and strangulation.

3. Stricture.

4. Volvulus.

5. Intussusception.

Calculi are composed of carbonate of lime and usually some carbonate of magnesia with it. They also contain more or less of the phosphates of lime and magnesia. These salts accumulate on the outside of a small stone, nail head or other foreign body in thin layers and they always begin to form around a nucleus. This is usually a piece of a nail, a screw, any foreign object such as a little pebble, etc. It must be something that does not dis- 
solve so that the salts of the intestinal juices will form around it until it grows to a very large size, and attains a great weight. Starting small in this way, the calculus forms a pocket, the lining of which becomes irritated, not enough to do any harm but enough to set up a sub-acute inflammation that runs into a chronic. The result is cell proliferation of connective tissue which forms a fibrous pocket which gradually becomes accustomed to the growing calculus. The calculus does no particular harm unless it rolls out of the pocket.

The "dust balls" usually form in animals feeding on mill cleanings from which they get dust and chaff of various kinds. These balls are light and usually round and of a black color,most always black. Sometimes these balls form in considerable numbers, a half dozen in one animal.

Stricture.-In this condition you must bear in mind that there is likely to be cell proliferation and inflammation. The formation of fibrous tissue thickens the wall of the intestines and destroys its dilatability, producing more or less stenosis. Some do not reduce the size of the tube while others reduce it to almost nothing. Strictures of this kind might produce a fatal obstruction in the bowels.

Volvulus.-This is sometimes called gut-tie or gut-twist. The word means a turning around. There are two ways in which the volvulus occurs: 1 . In one case a portion of the intestine seems to turn right around on itself, making from one to four turns; this is fatal by strangulation. 2. In the second we have a rupture of the mesentery as a result of more or less degeneration, and in this degenerated condition if the horse rolls, a portion of the bowel passes through the rupture making a twist on each side of the hernia. This causes strangulation. This is fatal.

Intussusception.-This is an invagination of a portion of a bowel into another portion of itself. This apparently should occur often, yet it does not, much to our surprise. It never occurs under normal conditions. In order to bring it about there must be a dilatation of a part, which is probably due to a minor amount of degeneration with relaxation, naturally causing the wall to dilate. When this takes place, the motion of the intes- 

tinal wall forces it right into the expanded part. It is seen most often in the ileum, a portion of which slips into itself or passes out through the ilio-cæcal valve into the colon. The cœcum sometimes becomes inverted into the colon. The result is obstruction of the bowel, and usually strangulation of both ends of the fold. This causes death through gangrene of the invaginated part.

Horses never recover from intussusception, but cattle sometimes do. Other animals do not recover. It is always fatal in dogs. People sometimes recover in the same way as cattle do. This condition is comparatively common in babies but rare in adults.

Semeiology of Intestinal Obstruction.-There are violent colicky pains and the case grows worse in spite of treatment. You may blunt the sensibilities with drugs but the disease grows steadily worse. Death usually takes place in 10-20 hours. There is a profuse perspiration; haggard countenance; occasional attempts to vomit; animal is inclined to sit on his haunches; strains as if trying to defecate; apt to stretch a good deal; always some flatulence; before death he becomes quite stupid.

Treatment.-We can only recommend general principles and the first is a careful examination of the animal in hand, especially in case of entire males. Stallions have been treated for colic when their trouble was hernia. When a stallion has colic always examine him carefully for inguinal hernia. Give oil ad libatum, a quart to start with and repeat in pint doses once or twice a day. Give rectal injections, cannabis indica, choral hydrate, and morphia and counter-irritation to the abdomen. Give eserine, arecolin or barium chloride.

Never neglect making a post-mortem examination on all obscure intestinal affections.

\section{EVERSION OF THE RECTUM.}

Definition.-This is a protrusion of the rectum through the anus. The mucous membrane may extend out from the size of a grape to several feet. It occurs in all animals. In parturient 
paresis in cows the rectum has been known to extend out 6 feet; in dogs I have seen it protrude $1 \mathrm{I} / 2$ feet.

Etiology.-Constipation is the main cause. A dry season in pasture with the streams dried up, dry feed, etc., too little water -these conditions cause constipation and they are responsible for the results of it. Diarrhœa will sometimes cause a prolapsed rectum. Empirical treatment of colic such as a piece of soap inserted in the anus, cauterizing it and thickening it, may bring above this cnoidition.

Treatment.-The tail must be bandaged and the protruding part be washed with warm water and milk, to which has been added laudanum $1 / 2$ ounce to the pint. Give the horse a liberal dose of chloral hydrate, 2 ounces for a 1,200 pound horse. This treatment will not paralyze but will blunt the sensibilities and relax the muscles (sphincter). Then try to replace the protruding part, using especial care not to wound the tissues. Use the palm of the hand. If the protruding portion is returned, inject more of the solution (milk and water). There are various means employed to keep the rectum in place, such as a rope twisted in the form of a truss; some take two crucial stitches in the anus, but if the animal strains at all these will burst. The best way is to keep a man with his hand upon the part until it stays in place. This is expensive but it is the only practical way. The congestion will subside in 3-4 hours, although it may take 10. The laudanum per rectum relieves the pain. You can use an ointment composed of petrolatum 8 parts and opium 1 part. The opium will overcome the peristalsis. In cows and dogs, when the bowels have been exposed for some time, the parts usually become gangrenous. In this case do not replace the gut, but excise the protruded part. Cut off the part $1 \frac{1}{2}$ inches outside the anus and then pull out the gut to get at the normal part. Cut off the remaining part so that the edges are normal tissue and suture the coats together, making the ridge on the inside, being careful to get the peritoneal coats in a position. Use the interrupted stitch. Before sewing sterilize the parts. After replacing pack with oakum. Give the animal sufficient opium to prevent defecation for three days. Then give a dose of oil and enemata. 



\section{HEMORRHOIDS.}

A hemorrhoid or bleeding pile is a little tumor of the mucous membrane due to interrupted circulation by pressure from fecal matter. The fecal matter pressing upon the gut produces passive congestion in a localized part of the mucous membrane. Constipation is always the cause of piles. A portion of the anal rose is sometimes caught in the sphincter and pinched. This interrupts the circulation and forms external piles. If the piles are on the inside they are produced by the hard fecal pellets. Internal piles become chronic. The feces rub them and make them bleed, hence the hemorrhage.

Semeiology.-The symptoms are bleeding and painful defecation. The horse will switch his tail and the fecal matter is covered with blood. In the human sometimes a severe hemorrhage occurs. The anus may be too small and contracted, making defecation difficult. If the feces are hard great harm may be done.

When internal piles become chronic there is so much irritation that it leads to a local inflammation of the rectal follicles an 1 runs through the first, second and third stages. If the abscess is not treated and cured it will ulcerate and perforate the bowel. In the horse the abscess is about two feet deep; in the hog 3-5 inches. It is not uncommon in hogs and in the human to see an opening on each side of the anus. In the horse the abscess may contain 3-4 quarts of pus. If the abscess is not opened it will rupture in from 10-30 days and become chronic. This is called an anal fistula.

Treatment.-First overcome the constipation. This is often very difficult, especially in the human. In the domestic animals, loosen the bowels with oil and then diet. Give dose (big) of chloral hydrate; after a half hour dilate the anus with a speculum and explore. If hemorrhoids are found, remove. Use antiseptic solutions for 10 days and keep the bowels open. Give salol. In case of external piles, remove in the same way, putting back only as a last resort. Sulphur ointments should be applied after each defecation. In the human astringents are relied upon but they are not much good. 
Imperforated anus sometimes occurs. The foetus may be born with the skin closed over the anus. In such case cut through and open up the rectum. Sometimes the rectum has to be sewed to the anus.

\section{RUPTURE OF THE INTESTINAL WALL.}

Rupture of the intestinal wall occurs occasionally but not as often as rupture of the stomach. It is usually caused by flatulence. The first attack very seldom ruptures a bowel and not very often the stomach, but repeated attacks lead to degeneration of some part which finally ruptures with much less provocation than may have existed some time before. The rectum is sometimes ruptured by reckless force used in back-raking.

Semeiology.-There are no diagnostic symptoms of this trouble but there will be pretty nearly the same symptoms as occur in case of rupture of the stomach. These are great nervous prostration; a small weak, rapid, hard pulse which finally gets wiry and imperceptible; animal sweats in patches; sits on his haunches. When the rupture is in the rectum there is usually violent straining and the animal dies from syncope. Death usually occurs in from 2-10 or even 20 hours.

The only positive proof of rupture of the bowel is the post mortem.

Naturally there is no special treatment but since there are no diagnostic symptoms you have to treat the case until the animal dies.

\section{ENTERITIS.}

Enteritis is an inflammation of either the small or large bowel. We consider enteritis as a true inflammation but there are authorities, writers and text-books that consider it more in the light of apoplexy. It usually comes on suddenly, runs a rapid course and terminates fatally. We do not believe that any cases of this disease ever recover but there are practitioners who claim to have cured many cases of it. Probably their cases were prolonged cases of colic, perhaps with local irritation just develop- 


ing prior to the removal of the cause. It often affects primarily the mucous coat and extends outward to the muscular coat and then the serous. The ordinary case runs it course in 6-20 hours and dies. The inflammation is so violent and severely acute as to produce rupture of the capillaries with more or less hemorrhage by rhexis. This results in extensive extravasations and great tumefaction. In the sheep, cow, and human cases of enteritis may recover, but in the horse I think never.

Semeiology.-At first there is dullness and slight uneasiness; the horse hangs his head, refuses his food, turns his head and looks around to one side; he soon develops fever; breathing accelerated; pulse increases in frequency and hardness; abdominal muscles are more or less contracted, for which reason the breathing will be more or less shallow; pain upon pressing the belly. The horse will lie down, roll, kick and sweat, but in lying down he usually goes down very carefully,-unlike a horse with colic. Fever runs up to 104 or 105 . The mucous membranes get very much injected and cyanotic.

When the horse passes feces, which may be in the rectum, at the time of defecation, you will notice that the lining is very. dark red. The animal develops a haggard, anxious countenance. After a severe case has been in progress for 4-5 hours, the pulse becomes rapid, small and hard, running somewhere between 70 and 100 . The horse ceases to lie down and walks continually if he is loose, with head elevated, eyes dazed, and stopping occasionally and sighing. When a horse sighs, it is almost a sure sign of a fatal termination. Horses never sigh except in the late stages of disease. The extremities get cold, horse gets stupid and loses sensibility to great extent. If the enteritis is in the small bowel the animal will sometimes vomit or try to; as death approaches, the mucous membranes get livid; he finally stops, stands quietly, pain seems to cease; pulse 100-120, probably imperceptible at the jaw; breathing rapid, shallow, and the expired air cold; he sweats in patches and the muscles tremble. We presume that gangrene has set in and the horse is only waiting to die from nervous prostration. I do not think that such a case lives long enough to die from septicemia. The horse stands as long as he can until weakness overcomes him and then 
he drops and soon dies. He may live 48-60 hours with this disease but an ordinary case will run its course and terminate in 6 hours after the first symptoms are visible.

Post Mortem.-Upon opening the bowel there is found patches of ecchymosis on the serous covering. The wall is greatly thickened in the average case $1 / 2$ to $3 / 4$ inches. The contents of the bowel are semi-fluid and bloody. This blood is from hemorrhage by rhexis. The thickened bowel wall will be infiltrated with serum and lymph. On the surface of the mucous membrane there will be a gelatinous coat of more or less coagulated lymph. The general appearance of the mucous membrane is very dark red, almost black. Other animals suffering from enteritis are usually very quiet, semi-stupid as it were. Pulse and temperature run about the same as in the horse.

Treatment.-It is difficult to prescribe rationally for enteritis for there are antagonizing conditions. Our best efforts, however, should be directed toward removing the cause in the hope that if we succeed the effects will cease. As a logical reasoning for this conclusion, we will say that whatever the cause of enteritis is if it is not removed, the horse will die. Consequently we want to get a free evacuation of the bowels and we treat as fo: constipation. Give oil in liberal quantities and eserine and arecolin. Give rectal injections, iiberal counter-irritation, alcoholic stimulants, give anodynes liberally, such as chloral hydrate, cannabis indica, etc., morphia not being so much indicated. In other animals besides the horse, opium is used freely, to quiet the bowel and suspend peristalsis and then give attention to reducing the fever. Such antipyretics as acetanilid are good, but in the horse the disease runs such a rapid course that we have not time for the drugs to act. We rely chiefly upon removing the cause and evacuating the bowels. It is always advisable to make a post mortem in a case of enteritis, especially so as to convince the owner that it was impossible to save the animal.

In mild cases such remedies as camphor, aconite and belladonna are indicated. Bleeding has been resorted to and has been found useless. 
, 



\section{PERITONITIS.}

Peritonitis is an inflammation of the peritoneum, the lining membrane of the abdominal cavity. It covers the mesentery and is reflected over the intestines. This serous membrane becomes inflamed and the inflammation runs close to pleurisy. The stages are the same. Two forms of peritonitis exist, acute and chronic. The acute form runs its course in about 10 days, the chronic may run along for months.

Etiology.-The most common cause is traumatism with infection. External violence, kicks from other horses for instance, especially those severe enough to produce ventral hernia; exposure to cold and dampness; strongylus armatus; starvation; old age (no appreciable cause known), -these are the main causes of peritonitis. In the third stage of peritonitis the effusion accumulates in the abdominal cavity and this condition is called ascites. Ascites may be due to chronic kidney and liver diseases without peritonitis, it being a dropsy from obstruction of the portal circulation or from defective secretion of the urine. It is sometimes tuberculous in cattle, and often follows castrations and abdominal operations.

Semeiology.-Pain is severe but the animal is comparatively quiet. Motion aggravates the pain so that instead of rolling and tossing and kicking, the horse is quiet. His back is more or less arched; abdominal muscles fixed; pulse and temperature up, temperature about 104, pulse 60-100; pulse hard and small ; pressure upon the belly causes pain. In the third stage the exudate is profuse. This is difficult to recognize as the distension of the belly is not marked. The legs swell; dropsical enlargement under the belly is usually seen but not always. The mucous membranes are hirst injected and later get pale; emaciation is rapid. The prognosis is usually unfavorable when the disease is extensive enough to be appreciable.

Post Mortem.-Fatal cases of peritonitis have ascites so that the post mortem will show much serum in the abdominal cavity. This varies in color from amber to red, depending upon the acuteness of the attack. The peritoneum is generally red over most of the surface, is softened and easily torn or punctured. 
In a chronic case you will usually find the heart, liver or kidney diseased.

Treatment.-We can prescribe for this condition in a rational manner. Control the fever with acetanilid, keep it down below two. Apply counter-irritants freely and repeat. A sinapism 2 or 3 times a day is the best local application. Give morphine hypodermically and give a stimulant with liberal doses of aconite, quinine and belladonna. Keep the animal quiet; bear in mind that motion aggravates the trouble as well as increases the pain.

Death from castration is usually due to septic peritoritis. Following a case of this kind you will usually have a swelling of the sheath and extreme redness of the wound. In addition to internal treatment and mustard on the belly, bathe the swollen parts freely and insert a disinfected hand up into the inguinal canal twice a day or so to make sure it is kept open.

\section{DYSENTERY.}

Dysentery is known as bloody flux. It is an inflammatory disease affecting the intestines and floating colon. The fever is of the same character as typhoid-a low prostrating form of fever. The discharges from the bowels are fluid, and contain an excess of mucous with some blood and pus and much fetor. These evacuations are accompanied by tenesmus of the rectum.

Etiology.-The animal has a predisposition, i. e., is weak constitutionally. The cause is probably specific. Some think that malaria is a cause. Dysentery is most commonly seen in cattle which are pastured on land overflowed with water or on hay cut from such land. The hay contains much sand and no doubt many germs, some of a specific character and these may be the cause of the dysentery. The feed should be examined for molds, animal parasites, and bacteria.

Semeiology.-Dysentery starts as a diarrhœa, but with a temperature of about 2 degrees. The prostration is greater than in diarrhœa. The coat is staring; almost complete loss of appetite; excessive thirst; tenesmus of the rectum; back arched, all four feet brought nearer each other; tail cocked, head low- 

ered; involuntary cramps and straining; fecal matter is largely a jelly-like substance streaked with blood and has a fetid odor. This is due to the degeneration of the mucous follicles in the rectum which are ulcerated. The molecular death (decomposition) causes the stench; sulphuretted hydrogen gas is formed. The tissues blacken a silver probe.

Prognosis.-There is a tendency to a fatal termination. The diagnosis should be guarded. If the disease lasts a week or ten days marasmus takes place, emaciation and nervous prostration develop very rapidly and death results from collapse.

Post Mortem.-The lining of the rectum is of a purple color and very much swollen; it shows numerous little elevations which upon closer examination prove to be ulcers. They look like papules. In young stock there would be general pallor of all the membranes.

Treatment.-Prescribe a complete change of food. This is all important. Give the best obtainable food and see that the water is pure. Give linseed tea with a little bicarbonate of soda. Laxatives are not indicated. Quiet the rectum by starch and laudanum injections with a 10 per cent solution of boracic acid added. Give opium internally by mouth and tonic doses of quinine. Salol and bismuth are good remedies. Allay cramps by hypodermic injections of morphine. Chloroform combined with opium and camphor are indicated.

It is all important to give attention to hygiene.

\section{DISEASES OF THE ABDOMINAL GLANDS.}

\section{LIVER-SPLEEN-PANCREAS.}

Diseases of the liver in the lower animals are less common than in the human, but when they do exist they are probably just as serious. The lower animals lead more natural lives and are less given to dissipation and therefore they are less given to disease. They are accustomed to eat what is given them and drink what is provided them and keep more natural hours. In the human this is very different. Many of the liver diseases in the human arise from alcoholic drinks in excess. 
The phenomena of the liver diseases are as follows:

1. Jaundice or icterus. This is a general yellowness of all parts of the body. It shows in the visible mucous membranes and in the skin, i. e., in the human.

2. Lameness-in very serious acute diseases of the liver the horse oftentimes is lame in his right forward quarter, Occasionally he has colicky pains.

3. Bilious diarrhœa, if the liver is working excessively.

4. Feces gray, in case of torpidity of the liver.

5. General listlessness.

6. Diarrhœa.

\section{CONGESTION OF THE LIVER.}

We recognize three congestions of the liver:

1. Active congestion, involving the hepatic arterial system,- - the nutrient system.

2. Passive congestion, irıvolving the venous or portal system.

3. Biliary congestion.

Bear in mind that there are two distinct circulations in the liver, the arterial and the portal or biliary.

In the hepatic arterial system we find that there is an increased flow of blood to the normal liver during the active stage of digestion. This is normal of course, but in disease there is an excess of blood at other times than that of digestion.

Etiology of Active Congestion.-Over-feeding on nitrogenous food is a principle cause. This makes an animal plethoric. This alone without any exciting cause is liable to develop hepatic arterial congestion at any time. Exercise, especially when an ani$\mathrm{mal}$ is in a gross condition during hot weather excites a condition of congestion. It is seen in horses that are pampered and very liberally fed and more or less idle. Over-feeding with too little 

work results in rupture of some of the smaller blood vessels of the liver and as a consequence hemorrhage. This hemorrhage is not sufficient to do any harm with a first or second attack, for the ruptured vessels heal and the clot becomes absorbed, leaving an anæmic patch, usually white. These patches can be seen on the surface of the liver under the capsule. These may vary in size from $1 / 4$ inch to $3 / 4$ inch in diameter. They are old chronic infarcts. These are common in old cattle and occasionally in horses.

In a more severe case rupture of the larger blood vessels occurs; the hemorrhage is greater, and if it is a third or subsequent attack, degeneration takes place to a greater or less extent. Consequently the infarction and hemorrhage are greater. Sometimes rupture of the capsule takes place with a fatal internal hemorrhage.

Etiology of Passive Congestion (Portal).-This depends mostly upon enervation, that is, nervous prostration of the animal. This is the principal cause of portal congestion. General enervation is seen in influenza, pneumonia and in other affections. The liver gets torpid and does not work as actively as it should. The bile is not secreted in proper quantity and biliary capillaries become congested, probably as a result of defective cardiac power. The congestion produces pressure upon the liver lobules, interfering with the outward flow of the bile through the minute bile ducts.

In case of pneumonia there is a different pathogenesis. There is a defective cardiac power. In all bad cases of pneumonia there is more or less obstruction of the arterial circulation in the lungs. This is especially so in the third stage, and the obstructed circulation dams up the outlet and leads to dilatation of the right ventricle, which in turn prevents the blood from leaving the liver. Consequently in most cases of pneumonia on about the fourth day we get considerable jaundice.

In chronic diseases of the kidneys there is an insufficiency of secreting substance, and consequently an insufficient secretion of the urine. This leads to ascites.

Biliary Congestion.-Biliary congestion occurs secondary to the active or the passive. It is a direct result of pressure upon 
the lobules and bile ducts. Then absorption of the bile takes place and we get a jaundiced condition.

Post Mortem.- In passive and biliary congestions the liver is darker than normal,-in streaks or in spots, usually in streaks. There are fine yellow markings. These are the surcharged biliary ducts.

Semeiology.-Listlessness is an important symptom. In active congestion pressure over the region of the liver will often cause pain. The horse or other animal will try to get away from one. Jaundiced mucous membranes are a sign; feces are usually dry, or after a period of constipation there may be a slight diarrhœa; mouth coated and has a soapy feel and a sour smell. In bad cases the animal grinds his teeth. In chronic cases the animal is hungry for salines. He will eat dirt, lick the wall, and chew the manger. Pulse and temperature will not vary much from the normal, unless the trouble is secondary to some other disease. In bad cases the appetite is poor and the animal gets unthrifty.

Treatment.-Treat according to the cause. If the hepatic disturbance is secondary to some other disease, treat the original disease. If the original trouble is in the liver, let the treatment center there. In case of a fat horse, let him fast. Give him a purgative, calomel and bicarbonate of soda. When you get results from these begin on some acid mixture, nitric acid. If there is no anæmia, give sulphate of soda night and morning for a week or two and regulate the diet and exercise.

\section{HEPATITIS.}

Hepatitis is inflammation of the liver. It runs through the three stages of inflammation and goes on to suppuration in the form of liver abscesses. This condition cannot be diagnosed during life.

Semeiology.-The symptoms are general unthriftiness; languor, animal unable to do much; the disease does not usually kill unless the abscess is large and ruptures internally. If the internal rupture takes place, the disease will terminate in pyæmia in fifty to a hundred days. 


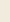



Post Mortem.-The capsule of the liver is thickened due to cell proliferation. The interlobular connective tissues is also thickened. Liver abscesses are usually associated with some other disease as glanders, strangles, etc.

\section{CHRONIC HEPATITIS.}

This is seen in one or two degenerations of the organ, viz. cirrhosis and fatty degeneration. Cirrhosis of the liver is the result of a subacute running into a chronic inflammation of the liver, affecting the interlobular fibrous connective tissue. The liver is large and hardened and the edges are rounded and thickened. The color is lighter than normal, more of a bluish color than the usual brick red. This condition is found in old animals which are starving because their teeth are too poor to grind the food given them.

Special Pathology.-Hypertrophy of the connective tissue produces pressure upon the biliary ducts and lobules and as a result we find yellow markings through the liver. The liver is brown in patches and the lobules are anæmic. The capsule is as hard as cartilage. This produces pressure upon the veins and portal circulation causing ascites. The word cirrhosis comes from the Greek kirros, meaning orange-yellow and refers to the yellow condition of the liver which is the result of the fibrous condition. This hardening may be applied to any organ but it more properly belongs to the liver. The hypertrophy of the connective tissue may produce atrophy of the parenchyma, that is, the epithelial cells which make up the lobules. It is often the primary lesion of ascites of old dogs.

Treatment.-This should be directed toward putting the animal in a better condition. Attend to its teeth, give him better and more easily digested food; give him potassium acid tartrate in his feed. 


\section{FATTY DEGENERATION.}

Fatty degeneration of the liver occurs in fat-pampered horses and dogs. The liver of a dog with ascites may be five times the natural size. In such a case the liver is large, soft and yellow and there is jaundice. The dog is fat and weak.

Treatment.-Reduce the artificial heat; decrease the amount of food and increase the work gradually. Sheep develop fatty degeneration if fed on non-nitrogenous food, such as white turnips. In such a case prescribe pea-meal and oats. The liver is large and yellow; miscropical examination shows fat globules in the liver cells; many of the lobules are transformed into fat. The animal may die from fatty degeneration of the liver attended by a rapid loss of flesh and strength following a prior period of thriftiness.

\section{ICTERUS.}

Icterus is a yellow condition of the whole system. This is caused by two factors: 1. Suppression (non-secretion of the bile); 2. Reabsorption of the bile. These two conditions may be differentiated by the presence or absence of biliary acids in the urine. These are glycocholic and taurocholic acids. When these acids are found in the urine they are a sure sign of reabsorption of the bile; when they are absent from the urine, it means that no bile has been secreted by the liver, that is, suppression.

Test for Biliary Acids in the Urine.-Put a dram of urine in a test-tube and in it dissolve a little cane sugar. To this add carefully so as not to mix a dram of sulphuric acid, holding the tube slanting so that the acid passes down under the urine. If biliary acids are present, a deep purple band will form between the urine and the sulphuric acid; if not, a brown band will form. In case the acids are present it is a sure indication of obstructed bile ducts and a consequent reabsorption. If the brown band is the reaction, then this is a sign of suppression.

Etiology of Suppression.-Enervation is an important factor. It occurs in the disordered hepatic circulation of cirrhosis, in tuberculosis, actinomycosis, fatty degeneration, etc, 

Etiology of Reabsorption.-This condition is due to obstruction of the bile channels. The obstruction may be:

1. Ductus choledochous-biliary calculus.

2. Tumefaction of the mucous membrane of the duodenum, probably from local inflammation, obstructing the outlet of the duct.

3. Stenosis-stricture and obliteration of the duct.

4. Tumors in the duct.

5. Pressure upon the duct from without.

6. Parasites. The ones most commonly found are the ascaris megalocephalus and the fasciola heptaica. The first is found in the horse, ox and sheep; the other found in any animal.

Semeiology.-In absorption of the bile there is general yellowness of all parts of the body. The icterus is much greater than when due to suppression. The urine is high-colored; feces gray and offensive; mouth feels pasty and soapy; smells sour; usually a loss of appetite, not always, in bad cases the temperature rises on the $3 \mathrm{rd}$ day; by the 5 th day the temperature will be up to 106; pulse increasing slowly, 60 by the 5 th day; itching of the skin, horse rubs himself raw in patches; skin gets thick and wrinkly; scurfy with desquamation of the epidermis. On the neck the skin lies in rolls size of finger. There is a distinct labored action of the right shoulder. There is constipation and the urine is scanty, syrupy and golden colored. The animal becomes emaciated, anæmic and finally dies from blood poisoning.

In suppression there is a less yellowness of the mucous membranes, and even this is pretty much the only symptom except in chronic cases due to absence of secreting substance, then there is general unthriftiness.

Treatment.-In reabsorption get rid of the obstruction. Purgation is indicated; for a horse of 1,600 prescribe six powders of calomel (1 dram) and bicarbonate soda (1 dram), one powder every two hours. Give these powders while the horse is fasting, 
then two hours after the last powder give 1 ounce of aloes. Let the horse have plenty of water, but nothing to eat. Twenty hours after giving the ball let him have soft feed and hay. When the purgation ceases give alterative doses of magnesia sulphate, or soda sulphate, night and morning for two weeks and then start in on quinine and nitro-muriatic acid with nux vomica.

$\mathrm{R}$

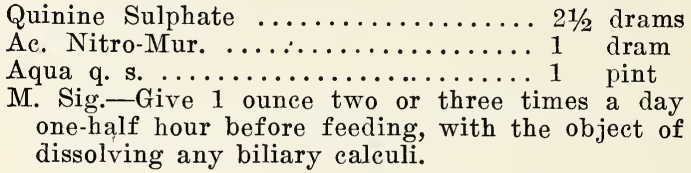

Do something for the itching. Acetic acid dilute (1 ounce) to a pint of water will make a good lotion. Wher applying, leave wet. In hot weather put on a sheet; in cold, a blanket. Tie the animal so that he cannot rub. The absorption of the bile into the blood poisons it and causes the itching.

In case sheep suffer from flukes, change to a higher better drained pasture.

\section{SpleEN.}

The spleen is subject to the various organic changes which take place in other tissues, such as congestion, inflammation, atrophy, hypertrophy, thrombosis, tuberculosis, carcinoma, melanosis, etc. The symptoms of disease of the spleen are negative.

Case of Lymphadenoma in a Horse.-The case was a draft stallion weighing 2,200 pounds, aged 5 years. He was sold in February and began serving in April and did good service up to June. The animal began getting languid and listless and in a month's time would not serve at all. He got cross and irritable, lazy, and emaciation set in. He ran down rapidly. He died the last of July a mere skeleton. The post mortem revealed a spleen weighing about 60 pounds. The organ was 6 inches thick and the surface was studded with numerous little tumors which appeared to be fibrous tissue. The horse was sold as a sound animal; suit was instigated to recover, but the owner lost. 



\section{PANCREAS.}

The pancreas is most commonly affected with fatty degeneration, but that is very seldom. One of the evidences is fat in the feces, probably due to faulty action of the pancreatic juice.

The symptoms are indefinite and are not diagnostic. The animal is anæmic and shows irritability ani dies riuch emaciated from collapse.

\section{CONSTITUTIONAL OR BLOOD DISEASES.}

A constitutional disease is one in which the whole system or blood is affected primarily, and if the disease localizes itself it does so secondarily. The exogenous diseases are contagious; the endogenous are non-contagious.

The word "contagious" refers to a disease that is due to a specific virus peculiar to the disease itself. The agent of the disease is called the contagium.

If an animal is affected with a contagious disease, the speci'fic virus of that disease inoculated into another susceptible animal will produce the disease. There are different means of inoculation:

1. Artificial-scarify the skin and introduce the virus.

2. Natural-exposure through the alimentary tract, respiratory, or through any mucous membrane or abraided surface, or accidental wound.

The contagium or specific virus in most contagious diseases is a known micro-organism-an animal parasite (protozoon) or a vegetable parasite (bacterium). In not all contagious diseases has the specific virus been found. In such cases we cannot call the causal agent a micro-organism, and yet we can reasonably presume it to be one or else the disease would not be specific.

As regards the difference between bacteria and protozooa, Sternberg gives a concise definition: The animal organism, that is a protozoon, receives food particles into the interior of the body assimilating the nutritious portion, and subsequently ex- 
truding the non-nutritious residue. A vegetable organism is nourished through the cell wall, which encloses the protoplasm, by organic or inorganic substances held in solution. This definition allows the vegetable organism both organic and inorganic food.

The discovery of the various agents of contagious diseases has produced the germ theory. This is as follows:

When a specific germ of a specific disease is inoculated into a healthy animal, it will produce that same specific disease and no other. The period of incubation is the time elapsing between the inoculation and the first physical signs of the disease. This may be called the incubative stage.

\section{VARIOLA.}

Variola is a specific disease affecting all higher animal life, but each species of animal has its own kind of variola and this is communicable to all other species. The human variola is the small pox; equine is the horse pox; bovine, cow pox or vaccina; then we have goat pox, pig pox, chicken pox, etc., but every pox is contagious.

Course.-Variola runs through four definite stages; viz. 1. Pimple; 2. Vesicle; 3. Pustule; and 4. Scab. Prior to the physical manifestations of the pox, there is probably a little fever, especially in the lower animals. In the human the pox is frequently fatal as is the case in black pox. In the lower animals the pox is mild. This disease is non-recurrent; one attack renders an animal permanently immune. Chicken pox may render some little degree of immunity in the human, and cow pox most certainly does. Vaccination is based upon the

1. Intercommunicability of the disease.

2. Non-recurrence of the disease.

History of Vaccination.-Dr. Edward Jenner, an English country practitioner, discovered the principles of vaccination. $\mathrm{He}$ was the first to notice that the milkmaids did not take the small pox, that they were practically immune. In 1768 he discovered the reason why. He examined cows and people and found that the cows frequently had kine pox. He began inoculating people 

with the serum of the kine vesicles in 1796 . He met with such success that he announced his discovery to the world in 1798. Vaccination was introduced into America in 1800 . It has proved so successful that the adoption of it has become world wide.

The specific cause of variola has never been found.

Etiology.-There is nothing known about the cause of pox. The disease has proved to be of a specific nature, and is more than likely due to some germ, but the causal agent has not yet been demonstrated.

Course.-Variola equina runs through four well defined stages, pimple, vesicle, pustule and scab. The pimple stage is short, about 24 hours. The vesicles contain serum which is thin, clear and transparent. About the second day it begins to get cloudy and the fourth day pustules form. Coagulation of the lymph and drying of the skin make the scab. This desquamates about the 21st day, leaving an oval shallow circular red pit. Equine variola usually attacks the mouth first, then spreads over the lips, cheeks, neck and shoulders and continues down the legs. Contagious stomatitis may be a form of variola. In case of infection from stomatitis use continuous applications of a 2 per cent solution of formaldehyde.

Treatment.-Wash the mouth with potassium chlorate, or sodium biborate solution. Keep a special watering bucket for the animal and keep him separate from the other animals. Thoroughly disinfect the stable after recovery.

Sheep sometimes die from variola.

Occasionally equine variola is enzootic. In such a case horses become infected after being fed or watered from contaminated food or water. , In 1877 an outbreak occurred in Montreal and the stable men in several places contracted the disease.

\section{ANTHRAX.}

Anthrax has various different names. The French call it Charbon; the Germans, Milzbrand; other names are Splenic fever, Woolsorter's disease, Putrid fever, Petaechial typhus; in East India it is called Loodiana disease, in South Africa, Horse sick.. ness, in the Highlands of Scotland it is called Braxy. Some writers call it contagious carbuncle. In the human subject, when 
anthrax is due to inoculation through a wound, it is called malignant pustule.

Anthrax is essentially malignant. As a rule it develops without any swellings or external manifestations, although there may be swelling. All warm blooded animals are subject to it. At one time it was thought that the birds and fowls did not have it, but this is erroneous. The disease usually runs a rapid fatal course, sometimes killing in 12-24 hours. In the horse many mild cases recover, but the recovery is slow. Some never fully get over the disease. A notable case of anthrax among fast horses, pacers and trotters was related by Dr. Baker. They may recover, but they never recover their former usefulness. They may have a wabbly gait.

Anthrax has been known from time immemorial. In the siege of Troy, animals are recorded as having died of anthrax. The people ate the animals and 60,000 of them died. It occurs epizootically and enzootically.

Etiology.-The cause of anthrax is the bacillus anthracis. It was discovered by two Frenchmen in 1850-Rayer and Davaine. The disease is communicable from animal to animal and from animal to people. The germs may be taken in through any channel, but most often by the mouth through food. The germs can enter any abraided surface, wounded skin, etc. In case of a skin wound the part should be excised. People can get malignant pustule through handling dirty rags, etc. Whether the animal dies from the disease or not depends upon the conditions of immunity. The course of the disease depends upon:

1. The natural immunity of the animal.

2. Strength of the culture, that is the amount of attenuation of the germs.

3. Number of germs inoculated.

The period of incubation is short-may be four hours. The disease is non-recurrent. One attack renders immunity to an animal. Pasteur proved this to be true and because of his investigations we now practice vaccination against it. $\mathrm{He}$ used broth as a culture medium and kept it at a temperature of 42 degrees $\mathrm{C}$. $\mathrm{He}$ found that the germs died in a month. When he introduced the broth into a healthy animal, it produced a consti- 


tutiona! fever. Pasteur experimented upon sheep, using two or three inoculations a week apart, and after the reaction of the fever, he found that the sheep were immune. This immunity usually developed after the second inoculation. Koch claimed that a hypodermic inoculation of anthrax would not immunize an animal from the natural infection; he further claimed that the germs were dead in a degenerated carcass, but he was not correct. There is no doubt that germs have infected people in carcasses two or three years old. Koch has immortalized himself, but he has made so many ridiculous announcements that he has lost caste amongst modern pathologists.

Horses get anthrax by grazing on lands flooded the previous spring. The streams are liable to contain a decomposing carcass which carries the germs. The disease may come through the food, through the flies, stings, etc.

Semeiology.-Some cases of anthrax develop external tumors. When the disease takes the form of apoplexy there is no swelling. In case of splenic apoplexy the animal dies suddenly.

The first thing noticeable is a high temperature, which in 3-4 days runs up to 106 . The visible mucous membranes become cyanotic, due to the disorganization of the blood. Weakness is progressive and rapid. A few hours before death the animal staggers, then goes down, dying from syncope.

Post Mortem.-The tissues show petechiæ or ecchymoses. The blood spots are local hemorrhages. The spleen is large and black, and full of coagulated blood. If the spleen is suspended the contents will gravitate, this is diagnostic of anthrax. Upon opening the heart the blood will be found to be black, thick, and non-coagulated. The liver will be congested, also the kidneys, and the fat around them will be ecchymotic. - These are the only lesions found post mortem. Anthrax is wholly a blood disease and it is a good plan to corroborate the diagnosis by a blood examination. Inoculate a small laboratory animal with some of the blood, spleen, kidney, etc., to see if anthrax develops. In making a post mortem, always remember that the disease will attack the doctor as well as any one else. Use rubber gloves, keep the flies away. Do not allow the carcass to be skinned, burn it. If it must be buried, cover it with unslaked lime and 
5 feet deep. Otherwise the earth worms will bring the germs to the surface and infect the animals grazing near by.

Treatment.-If the case is the first one diagnosed, then treat the fever and when the animal dies, as he surely will, hold a post mortem. If the post mortem confirms the diagnosis, then quarantine against the source of infection. No medical treatment will touch anthrax. You can vaccinate, however. Get the printed directions which come with the virus and follow them carefully. Make sure of your diagnosis before saying anything. Inoculate laboratory animals, and if they die examine their blood microscopically. If it is anthrax you will find the bacilli in large numbers. The fat around the kidneys is the same as in Texas fever. The blood of anthrax is not like that of any other lisease; it is like tar

\section{RABIES.}

The name rabies is derived from the Latin rabere, to rave. In the human subject it is called hydrophobia. This word means dread of water, but the disease is the same as in any other animal. Water sets the human patient into paroxysms, but so would anything else. Rabies is a peculiar contagious disease. It presents its symptoms through the nervous system in the form of paroxysms which are invariably fatal. All warm blooded animals are subject to it. The virus seems to live in the saliva, but an emulsion of brain or spinal cord of a rabid animal will produce the disease when inoculated into another animal. Other parts, such as the blood, feces, etc., when inoculated, give negative results. The rabid virus may be absorbed through a wound. It is usually easy to trace the origin of the disease. We regard the history of every case important.

Etiology.-It was formerly supposed that dogs go mad by reason of a spontaneous development of certain conditions, such as deprivation of water, confinement, ungratified passion, etc., but recent investigations show that rabies may occur in any dog and in any place, the one thing needful being a bite from a rabid animal. Bites around the head are much more dangerous than when located in other places. Pasteur states that only one out 

of five bitten by rabid animals develops the disease. So far as the specific virus is concerned, we do not know much about it, but the agent must be large for it can be filtered out from the saliva. The so-called Negri bodies are a phenomenon of this disease. In 1903 Negri discovered the small bodies in the nervous tissues of rabid animals. They can be seen under the scope in the form of little granular oval or round masses which stain red. They are found in the substance of the brain, especially in the horn of Ammon. Investigation of these bodies is being conducted in many laboratories and all reports do not agree as to the importance of these bodies from a diagnostic standpoint. They have been found in animals not diagnosed as having rabies, but such diagnosis was not positive.

Prof. Negri, Dr. Lagorio (Chicago), and Dr. Frothingham (Boston) have done much work on the subject of rabies. Dr. Frothingham claims that these bodies are a positive evidence of rabies.

So far as the deprivation of water is concerned, any animal will develop a fever when deprived of water. Statistics prove that we have more rabies in the fall and winter than in the summer and the temperature has nothing to do with it.

The period of incubation may be as short as a week, and it runs an indefinite length of time. Rabies may be traced to a bite 6 months past. The incubation period averages 28-35 days. The periods are the same in all animals.

Semeiology.-Horse. The first thing noticed is nervousness. There is an unnatural twitching of the muscles of the face and the patient is irritable, inclined to bite his attendant. He drinks slowly and on the second day cannot drink at all. He shakes his head, breaks the bucket, etc., and does not eat because of the paralysis of the muscles of deglutition. The horse grows constantly more restless and has spasmodic fits of kicking; makes attempts to bite, but does not really do so.

According to the severity of the attack paralysis sets in sooner or later and the animal staggers until he goes down in a fit of unconsciousness.

The bitten part seems to itch and the horse rubs his wound against the stall. If the wound is on a leg, the animal nibbles the 
part, bites it, tears it, wounding himself severely. There is no fever, the animal dies comatose. Death occurs about the fifth day. The course and termination of this disease indicates that it is specific. The symptoms in other animals will be taken up by Dr. White and Dr. Wright.

Differential Diagnosis.-There is no danger in making a mistaken diagnosis for no other disease resembles it. In the horse cerebro-spinal meningitis has some similar symptoms. In the dog acute indigestion must be differentiated. Dogs vomit in indigestion, but not in rabies. Because a dog bites, he is not necessarily mad. He will bite in case of worms, brain trouble, etc. In dog practice it is sometimes difficult to make a diagnosis between the diseases of the brain and those of the stomach.

Treatment.-Rabies is always fatal, consequently medicinal treatment is useless and the only thing is preventive treatment.

Pasteur discovered the efficacy of inoculating an animal with attenuated virus. He inoculated chicken broth with attenuated saliva of a rabid dog (saliva was old) and it gave no results. Then he inoculated a rabbit with an emulsion of the brain of a rabid dog; then he used the inoculated animal to inoculate the next and so on until he had inoculated 50 . He found that the 50th attenuation was very mild in action, and when an emulsion from such an animal was used, it was slower to act. He used attenuations of the 50th degree up to the 12th, making the inoculations 3 or 4 days apart, and this course of treatment produced immunity.

The Pasteur treatment as carried out today is practically the same. An emulsion of spinal cord of a rabbit is used and kept in the incubator for 3 days at $70^{\circ} \mathrm{F}$ when it is sufficiently attenuated. This attenuated virus is used for the inoculation. Dr. Lagoria inoculates for 21 days, using a 3rd day attenuation for 3 days, 2 nd day attenuation for 5 days, etc. If the bite occurs on the face or neck, the treatment should be given immediately. In all cases it should begin by the 3 rd day. 


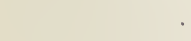





\section{GLANDERS.}

Glanders or farcy (French, La Morve; German, Rotzkrankheit) is a specific inflammation especially characteristic of the equine species, horses and asses. It is communicable to the human, dogs and cats. It has recently been found that it could be inoculated into the bovine species. Bovines are generally considered to be immune. Glanders is considered a fatal disease. Some mild cases can possibly be cured, but these are infrequent.

External glanders refers to the specific eruption of the Schneiderian membrane; internal glanders attacks the invisible mucous membranes such as those of the larynx, trachea, bronchi, etc., and the lungs in the form of interstitial pneumonia, the liver and internal lymphatic glands.

Farcy is an eruption of the external lymphatics and ducts. It is spoken of as cutaneous glanders. In all fatal cases of either glanders or farcy the one runs into the other. A horse seldom dies from the one form of lesion without the other appearing. The disease runs two courses, acute, and chronic (subacute). The acute form kills in 3-6 weeks, although the horse might run along for a longer time; the chronic form lasts indefinitely.

The disease is as old as anthrax. It was described under the head of malleus in the fourth century. The French wrote about it in 1618. LaFosse, in 1749 said it was a spontaneous disease and described it as ulceration of the mucous membranes. It exists quite generally in the uncivilized world as well as the civilized.

Etiology.-The specific germ of glanders called the bacillus malleus was discovered by Loeffler and Schultz in 1882. These men were members of the German board of health.

The germs are found in the discharges of glanders and farcy. With them are also found many other bacteria.

Semeiology.-The earliest symptoms are fever and diabetes insipidus. These symptoms are not usually noticed unless the horse is a valuable one and is observed to miss a meal or two. The horse may show so marked polyuria as to partially flood the stable. He has a good appetite, but in spite of it he loses flesh rapidly. He is not sick long before a nasal discharge begins in 
the form of a red limpid serum mixed with mucus. After a few days this becomes muco-purulent and streaked with blood. If you look into his nose you will notice a red local swelling elevated at least $1 / 4$ inch. This is the pustule of glanders, and when it ruptures the hemorrhage causes the pus to be bloody. The mucus from the nose is of a catarrhal nature, coagulated and starchy. It has no actual diagnostic appearance, but it is suspicious because of its resemblance to melted butter. This sticky discharge accumulates around the rim of the nostrils and dries there more or less. This interferes with the respiration. The horse blows his, nose and a stringy discharge flips up and lodges on the side of the face where it attracts dust and dirt. The glandered horse always has a dirty face. The stall also will be just as dirty. You can always tell a glandered horse's stall.

In the course of a week the submaxillary lymphatics will swell. They catch the debris and germs. As a rule they do not suppurate. This symptom is not diagnostic, for you get the same in chronic catarrh.

The pustule in the nostril ruptures, eventually ulcerates. The ulcer has an elevated, ridged edge with a depressed center. Ultimately the whole mucous membrane of the nose has a mouse eaten appearance. In a mild case, especially if the pustules form high up in the nostril, the air passages become constricted and we get wheezy breathing, but this is not diagnostic.

Cough, accelerated breathing and pneumonia are the next symptoms. The pneumonia is interstitial because it involves the interlobular connective tissue. The lining of the bronchi becomes ulcerated. In the course of a week we get cuticular manifestations, particularly in the inguinal region and at times in the pectoral. The legs are doughy and the nodular swellings discharge and ulcerate. These ulcers do not heal, but remain in a state of continual discharge until death.

In such a condition the horse is dejected, hangs his head and looks miserable. The discharge from the nose may become somewhat offensive in a late stage. The animal dies from collapse. During the whole course of the disease the fever stays up to $102^{\circ}$ to $104^{\circ}$ or even more. The pulse is increased in frequency and decreased in size and strength. 
. 

In subacute glanders a chronic discharge from the nose is usually the first thing noticed. It is of the same character as that in the acute only not so exaggerated. The case runs along with no other symptoms except the discharge and the enlargement of the submaxillary lymphatics. Such a case may keep up indefinitely if well cared for. But during all this time the horse is sowing the germs of the disease everywhere he goes. The termination of a chronic case is a sudden development of the acute form. This comes from exposure such as an animal would get on board ship or in the army, or even poorly ventilated stables may be a cause or hardship of any kind, exposure to a cold storm, etc.

Acute Farcy.-This form of glanders starts with abscesses in the lymphatics. They begin as small nodules about the size of a hazel nut and suppurate, rupture, discharge and ulcerate. The legs swell and eventually the eruption involves the greater portion of the body. The condition grows worse rapidly and after 2-4 weeks the case runs its course and acute glanders develops.

Chronic Farcy.-In a mild form the inflammatory areas on the leg become indurated while small nodules spring up in other parts. The course is slow. Many of the ulcers heal, leaving hairless scars. Sometimes the farcy buds come up on the head and neck pretty thick; they heal and leave scars. Such a case may continue for years until some unfavorable condition develops to produce a severe exacerbation.

Besides the conditions previously mentioned, severe purgation may bring on a sudden development of acute glanders. In this climate chronic glanders runs its course in about 3 years, but in North Dakota and Montana it may run along for 10 years or more.

How is the disease carried? The usual port of entrance is thought to be the alimentary tract. The virus may be absorbed from any mucous surface or through any abrasion of the skin. Feed boxes, drinking places and hitching posts are sources of infection. A glandered horse is prone to rub his nose and the sticky discharge therefrom contaminates everything it comes in contact with. Dogs discharge much more freely than the horse. They carry the glanders germs from one place to another. 
The period of incubation of glanders may be as short as a week. This is difficult to estimate for we do not know what time the case was infected.

Treatment.-All kinds of treatment have been tried but they are all a failure. It is useless to attempt to give medicine and it only endangers the life of the attendant. Destroy all glandered horses. In order to do this you must get the consent of the owner or else fall back upon the state. The state law must be given liberal consideration. You cannot take a man's property from him without due process of law. In case the owner objects, go to the state's attorney and find out what action to take. Quarantine the premises in the interest of the public health if the owner is obstinate. The quarantine can be maintained indefinitely.

The main trouble in glanders comes with the handling of suspicious horses. In a stable where one or two animals have the disease and the rest are exposed how will you protect the public? The first thing to do, if the doctor is working under the state law and has the power, is to quarantine the place. If the doctor has not this power, then let him report it to the state officer. In most of the states there are laws regulating the disposition of animals affected with contagious diseases and glanders is one of these diseases. Glanders, foot and mouth disease and Texas fever are recognized and handled officially. Most states require practitioners to report all cases of contagious diseases. In Illinois there is a $\$ 500.00$ fine for failing to report to the state veterinarian any contagious case.

After the stable has been quarantined then test the animals with mallein.

Mallein is an albuminous serum. It varies in strength but that prepared by the United States Bureau of Animal Industry is taken as a standard. A dose of that which is made in Washington is 1 cubic centimeter, about 16 drops. This comes in bottles ready for use and is injected under the skin in the side of the neck. The object of making the injection in this place is to select a place where the swelling will be visible. The hair should be clipped off and the skin washed before the injection is made. This should be done about ten o'clock at night. The 


temperature of the horse prior to the injection should be taken three times, morning, noon and night. The horse should be in good condition and the temperature should be taken under favorable circumstances. Do not take the temperature soon after drinking cold water but before eating or drinking and at a time when he is not warm from exercise or work. He should not come from an over-crowded or ill-ventilated stable in such a case if ever you want a normal temperature. After making the injection take the temperature the next morning at 7 o'clock, then every two hours during that day and if necessary through the night. If the horse has glanders you will get an elevation of temperature from 3-6 degrees. The maximum temperature will usually be found about 14 hours after the injection. The temperature, as a rule, in these cases rises slowly but persistently. It keeps rising for $24-30$ hours. It will then fall within 2 or 3 degrees of normal and stay there, forming what is called the rainbow curve.

At the point of injection there will be a swelling varying in size from 3-9 inches in diameter. It is usually about $3 / 4$ inch thick. Radiating, swollen lines will be seen running from it. These are sometimes 3 or 4 inches long, may be a foot and they are apt to run downward toward the point of the shoulder. This swelling is intensely painful.

The constitutional effects of the mallein test are as follows:

The horse hangs his head, loses his appetite and is disinclined to move and sometimes has minor chills. This depression is so well marked that it is an important symptom. It indicates that the diagnosis of glanders is unquestionable. A great many cases have been tested, destroyed and posted and I have never heard of a case with these symptoms of depression with the febrile reaction and local swelling at the point of injection proving to be anything else but glanders.

Of course there will be varying degrees of severity of reaction from the test. The temperature may run along to 105 or 106 , and there be present swelling, soreness and depression, and there would be no question of its being glanders. But suppose the maximum temperature is 103 , swelling is 3 inches in diameter, no soreness, no radiating lines, no depression,- then this is a 
suspicious case. You cannot say that he has or has not glanders. Every animal should either be named or marked on the temperature blank. Mark the last case as suspicious and test him again after 30 days.

In case the horse has not glanders the mallein test will give no results at all, there will be no elevation of temperature. However there may cases come up that will give a little temperature, possibly a degree or two. There will be a slight local swelling, but no radiating lines. Before night these symptoms will be gone. You would declare such a case sound.

Disposition of the Animals.-The strong reactors should be destroyed, the mild ones quarantined and tested again at the end of one or two months. The non-reactors should be taken out to a clean fresh place and be kept separate from the others. When the tests are made again at the end of the month you may find some that give a marked reaction showing that the disease has developed in that time. On the other hand some will react suspiciously at the first test and then when tested again show clearly that they were not infected. Such cases should be tested several times. It is important to protect a man's horses as far as possible. Do not kill any more horses than is absolutely necessary.

The failure to react on the part of some of these animals that are tested two or three times creates the impression in the minds of many that the mallein test immunizes the animal. In some mild cases the mallein seems to act as a curative and the animals become sound again, living along indefinitely, while in others the addition of a little more poison than they already have in their system precipitates the disease to sudden termination. This point is being debated throughout the world today by prominent veterinarians, but we aim to be conservative and protect the public. If you are acting under a state veterinarian who is inclined to be radical, argue the point with him and if you feel that he is unreasonable, more so than you can endorse you better resign than be caught by his mistake.

Thorough disinfection must be given a stable where a glandered horse has been found and destroyed. This is much easier done where the barn has a good floor. Clean out the stable thoroughly, wash the stall, etc, Fill a large bucket with boiling 


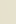



water, put in three or four ounces of carbolic acid, one-half ounce of bichloride of mercury to three gallons of water. Then with an old broom scrub the stall. Wet it good and let it soak a while then scrape out. Do this two or three times. If the stable is tight enough to use formaldehyde and fumigate, do so. Shut the stable up tight, suspend a number of sheets in it and spray the formaldehyde over the sheets, starting at the far side from the door. Do the work very rapidly and back out toward the door as you work. Let it stand five or six hours in this condition, then open the windows and doors letting in all the fresh air possible until it is thoroughly ventilated. Then whitewash extensively with fresh slacked lime and sprinkle the floor liberally with a ten per cent solution of coal tar emulsion. Where the glandered horse has stood take out the manger and burn it.

Some think that the life of the germ is only a few months, that it will die with time. This is recognized as correct for outdoors-for water troughs, fence posts, hitching posts, specially it exposed to winter weather with alternating thawing and freez. ing, etc. But inside this is not so.

The harness used on glandered horses should be cleaned very thoroughly and the bits boiled.

Mallein Test.-We have occasion to have great confidence in the Mallein Test. If the horse has an elevation of temperature to begin with we may get a reading of thermometer that will not tell us much as regards the diagnosis. But if the temperature is normal and the reaction is definite, say 3 or 4 degrees above normal then the case is one of glanders. If the temperature rises 3 degrees and depression is slight then do not kill the animal but test again a month later. Suspicious cases should be retested.

If thought advisable, confirm the mallein test with some other such as the Strauss test or the agglutination test.

Strauss Test.-Inoculate under the skin or into the peritoneal cavity of the male pig some glander's pus. In three days the pig will show swelling of the testicles which are sore under pressure if the pus is specific of glanders. In thus inoculating the pig other germs will no doubt be introduced into his system so that the pig will die probably anyway from an infection but the swelling of the testicles is the diagnostic point. 
Agglutination Test.-This test is a new laboratory method. It is based upon the theory of the agglutination of germs by an infected serum. The serum is first diluted and then inoculated with a suspension of the dead glander's germ and after a definite length of time agglutination of the germs takes place. This reaction can be seen in a test tube, in the test tube the germs make a floculant white precipitate at the bottom of the tube.

Post Mortem.-Suppose we have a case which shows no evidence of the glander's lesion. At post you will probably find an interstitial pneumonia or glanders abscesses in the liver or lymphatic glands. Many cases will show internal evidence of glanders which were wrongly diagnosed during life.

Order the animals which react strongly, killed, that is, those that have a marked temperature or swelling. Mild cases usually react less and less to each test. They should be held over and tested again. Never inject mallein into an animal when there is fever present from some other cause than the glanders infection. All suspicious cases should be quarantined and tested again. The mallein test will hurry the termination of an acute case.

In Montana glanders is mild; horses live for years with the disease and scatter the germs everywhere. Canada gets her glanders cases from Montana and this has compelled her to refuse to admit any reactor into her boundaries. Reactors should be kept in continuous quarantine. The owners can put them to work, but they must be kept out of the way of other animals.

The Board of Live Stock Commission of Illinois can make rules that are just as binding as laws. The old laws put the quarantined cases in the barn and kept them there but according to the present regulations the horses can go on the public highways providing they are inspected every week at the owner's expense. The veterinarian can charge $\$ 2.00$ for every 10 horses or fraction of ten. The object of these inspections is to catch the cases that develop early. If the owner works his horse after he has been ordered to hold his animal in he can be arrested. The time limit of quarantine is usually 90 days if nothing develops, but the quarantine runs indefinitely.

The law says that the owner must carry his own waterpail; 
-

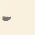



that he cannot water his horses at public watering places; that he cannot use other stables or tie to hitching posts, etc.

\section{DIFFERENTIAL DIAGNOSIS BETWEEN GLANDERS and CHRONIC CATARRH.}

Ulceration of Schneiderian

Membrane.

Discharge continuous.

Discharge usually bilateral.

Discharge resembles melted

butter.

Doscharge odorless unless there

is extensivet necrosis of

the turbinate bones.
No ulceration at all.

Discharge intermittent.

Discharge usually unilateral.

Coagulated in chunks, not gluey.

Discharge always fetid.

\section{RECORD OF FIVE CASES TESTED WITH MALLEIN.}

The first three cases occurred in Dr. Vernon's practice. M.

Temperature before

$$
\text { injection.....10:00 p. m. } \quad 101 \quad 100 \quad 101
$$

Temperature after

Case No. 1. Case No. 2. Case No. 3.

\begin{tabular}{|c|c|c|c|c|}
\hline injec & 6:00 a. m. & $102: 5$ & 100 & $102: 5$ \\
\hline 6 & 8:00 a. m. & $103: 6$ & 102 & 104 \\
\hline 66 & 10:00 a. m. & $103: 4$ & $102: 4$ & $103: 6$ \\
\hline 66 & 12:00 m. & $103: 4$ & 102 & $103: 4$ \\
\hline 66 & 2:00 p. m. & $103: 2$ & $101: 8$ & $101: 8$ \\
\hline "6 & 4:00 p. m. & 102 & & $101: 2$ \\
\hline 66 & 6:00 p. m. & $101: 6$ & & 101.2 \\
\hline
\end{tabular}

The first case developed a swelling at the seat of injection 8 by 10 inches. There was no swelling the next morning and the horse showed no depression.

In the second case the swelling was slight and there was no depression.

In the third case was a swelling 5 by 10 inches with no depression.

The question arose what to do with these animals. As the temperature and swellings were not typical it was decided that they should be liberated. 
The next two cases were under Dr. Schneider's observation.

Temperature before

Case No. 1-Gray. Case No. 2-Roan.

\begin{tabular}{|c|c|c|c|}
\hline \multicolumn{4}{|c|}{ Temperature before } \\
\hline inject & . $3: 30$ p. m. & $99: 9$ & $100: 6$ \\
\hline 66 & $7: 00$ p. m. & 101:6 & $101: 5$ \\
\hline “6 & 10:00 p. m. & $101: 4$ & $100: 8$ \\
\hline \multicolumn{4}{|c|}{ Temperature after } \\
\hline inject & . 6:00 a. m. & 104:1 & $100: 3$ \\
\hline 66 & $8: 00$ a. m. & $105: 8$ & $102: 6$ \\
\hline 66 & $10: 00$ a. m. & $105: 8$ & 104 \\
\hline 66 & $12: 00 \mathrm{~m}$. & $105: 9$ & $103: 3$ \\
\hline 66 & 2:00 p. m. & $105: 3$ & $102: 4$ \\
\hline 66 & 4:00 p. m. & $105: 7$ & $104: 2$ \\
\hline 66 & 6:00 p. m. & $105: 2$ & $104: 2$ \\
\hline 66 & 8:00 p. m. & 105 & $103: 3$ \\
\hline 66 & 10:00 p. m. & 104 & 104 \\
\hline 66 & 7:00 a. m. & -105:1 & $103: 8$ \\
\hline
\end{tabular}

The first case developed a swelling 4 by 5 .

Second case developed a swelling 3 by 4 .

Botk of these cases showed great depression the day following the injection and the next day also. It was decirled that they had the disease and they were destroyed.

\section{STRANGLES.}

Strangles, Gourme (French), Druse (German), is a contagious disease peculiar to the horse. Sorne writers describe it under the name of Coryza Contagiosa Equorum. The specific germ was described by Schultz in 1888 and he called it streptococcus equi. Horses of all ages may contract the disease but it is generally known as a colt disease. It is non-recurrent, but possibly in rare cases it may attack an animal the second time.

Strangles runs two courses, the regular and the irregular. The irregular course used to be a disease called Pastard Strangles, but this name has become obsolete.

In its nature strangles is a pyogenic fever. No other species of animal is ever attacked. The regular form manifests itself in abscesses about the throat, in the submaxillary space, in the glands and lymphatic vessels about the head. It runs a course of about a week or two when the abscesses will have ruptured spontaneously and healed benignly. The abscesses usually discharge for about a week.

Inoculation with pus from these abscesses will not always 
$\checkmark$ 

produce the disease, but cohabitation of the diseased and healthy animals seems to transmit the infection. It cannot really be explained how the infection is carried. It may be from the pus or it may be from the expired air or discharges of the diseased animal.

Etiology.-The streptococcus equi is the cause. The period of incubation is from one to two weeks.

Semeiology.-There is at first a high fever which is soon fo!lowed by loss of appetite and swellings around the throat and branches of the lower jaw. The abscesses may be numerous or few, large or small. Regularly they occur around the head and throat. If the retropharyngeal glands are involved the dyspnœa may be dangerous and death from asphyxiation occur. The mortality is not high. About 99 out of a 100 recover, but occasionally one will die. The death depends a great deal upon the environment and the weather. Wet and cold are unfavorable to the sick animal.

Strangles usually starts with fever and a discharge from the nose, and there may be some cough. At first the pulse is rapid and in a few days it becomes weak. The mucous membranes become injected, and if the abscesses occur around the throat and maxillary glands, the deglutition is apt to be interfered with. In a few days they will rupture spontaneously unless they are too deep-seated. Then the fever subsides, the appetite returns and rapid recovery takes place. The temperature may start out at 106 but in 4 days go down to 104, at which place it stays until the abscess ruptures, when it returns to normal.

In the irregular form the character of the fever is the same but the abscesses form somewhere else than around the throat, either internally or externally. They are often seen in the liver, spleen and lymph glands and the pectoral and inguinal branches of the lymphatic ducts. They may break out about the legs. If the abscesses are superficial and can be opened or ruptured the animal will make a good recovery, but if the abscesses follow one another then the animal may not get well for six or eight months. If they rupture internally the case may be fatal. The pericardium and occasionally the heart itself is often the site of these ab- 
scesses. They sometimes occur on the pleura and rupture into the pleural cavity.

Treatment.-Strangles must run its course-it cannot be aborted. Rational treatment will help to produce a favorable termination. In this and every other febrile disease the patient should be housed and kept warm. Let the food be laxative. Crushed scalded oats and bran are good. Apply linseed poultices to the abscesses to soften them and repeat the application night and morning. Open the abscesses, do not let them rupture. They should be opened 3 or 4 days after they start. Syringe them out and still keep on the poultice. This will hasten the recovery. After 2 or 3 days stop the poulticing but cover the sore part if the weather necessitates it. If dyspnoea is accentuated, perform tracheotomy.

As to medicine, give quinine and whisky every 4 hours. If the temperature is around 104 or 105 give small doses of acetanilid. Keep the temperature at 104 or less. As soon as the abscess has been opened or has ruptured put the patient on tincture of iron. For the irregular case, if you can make a positive diagnosis, give quinine and whisky, but if the case runs for a long time use anti-streptococcus serum. This may effect a recovery. "It would be a good plan to give all suspected cases a prophylactic dose of the serum.

Post mortem reveals nothing more than the abscesses.

\section{BURSATTI.}

Bursatti is the name of a disease which produces inflammatory swellings in the skin with raw suppurating surfaces. These are called kunkurs. The name "bursatti" is derived from the East Indian word burus, meaning rain, and the disease is spoken of as rain sores. Up to the present time it has proven to be incurable. It may yield for a time but the kunkurs will return. The disease is found mostly in the South and the outbreaks occur in the summer. It is not considered contagious from horse to horse, but rather infectious, two or more getting it from a common source. The inflammation is located in the subcutaneous tissue, and the tumors may be found in the internal organs, In- 


oculation with the serum from the ulcers does not seem to produce the disease. The cause of it is not really known. It is believed to arise from filth. It seems to occur in horses which have not been kept clean and whose harnesses have never been cleaned. The harness rubs against the skin until the surface is raw and an ulcer forms. If the ulcer heals it will eventually return. This condition interferes with the welfare of the animal and he runs down in flesh, getting thin and worthless. In India it is considered a hereditary disease and animals so affected are condemned for breeding purposes. The raw surfaces of these ulcers itch a great deal and the itching seems to be mainly around the outside of the ulcer, which is slightly raised. Two or three of these ulcers may start in the same region and by spreading they will become confluent.

Treatment.-The hygiene is considered important. See that everything is cleaned up and prevent the harness from rubbing the sores. The horse should have a liberal diet and be fed on good nutritious food. Prevent the horse from scratching or biting the raw surfaces if possible. If called early extirpate the inflammatory area if it is not too large. In addition to this give rational treatment. The sores of bursatti are similar to the socalled summer sores although there is a distinction. Neither will endure winter.

The following is a summer sore lotion which seems to be effectual in some cases:

$\mathrm{R}$

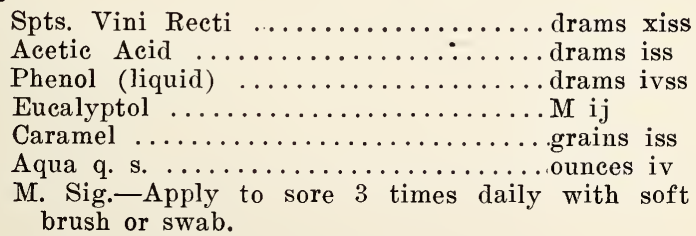

To prepare dissolve the eucalyptol in the alcohol; dissolve the caramel in $\mathrm{H}_{2} \mathrm{O}$, then add the acetic acid and phenol and shake. 


\section{BOTRYOMYCOSIS.}

Closely related to bursatti is the disease called botryomycosis and it is often difficult to differentiate them. In botryomycosis the tumors which go on to ulceration are due to the invasion of a microorganism called the botroyomyces. The tumors which are formed are inflammatory growths, often of very large size. Scirrhous cord may be caused by this microbe. The tumors should be removed with a knife and all the parts of the growth thoroughly cut out as the botryomyces invade the outer zone of the tissue. In the discharge from the abscess colonies of the microbe may be found but these are the older organisms and are not as active as those in the outer parts of the tumor. The knife seems to be the only treatment, although the summer sore lotion has been tried with some results. The knife should always be applied to scirrhous cord.

\section{CORN STALK DISEASE.}

Horses and cattle die from a disease known as corn stalk disease when turned into a stalk field in December and January. In these months the disease seems to be prevalent. The animals eat the nubbins and stalks and suddenly they come down with an affliction and next morning are found dead. It is supposed that they get the disease from the corn stalks. Animals that get a mild attack do not die so early but become delirious or at other times may become stupid and finally die in a comatose condition. This shows that the disease is due to some toxin. All animals which contract the disease die from it sooner or later.

Prevention is better than cure. Remedial treatment seems to be useless. Prevent by keeping the stock out of the fields. Cut the stalks early enough to make good fodder and there will be no corn stalk disease.

\section{SURRA.}

Surra is a constitutional disease of the infectious type. The infection is not carried from animal to animal but is due to the 

introduction into the animal of a hæmatozoon known as the trypanosoma Evansi. This parasite has a long whip-like body with a tail something like a flagellum. The parasite was discovered by Evans. The organisms may be taken into the body through the medium of the food and water, but it is believed now that they are inoculated into the host by some certain insect, such as a certain kind of fly or mosquito. The disease affects the horses usually in the low, swampy regions. It is called trypanosomiasis. From the alimentary tract the parasite enters the blood and more or less disorganizes it, producing great debility, emaciation, fever, capricious appetite and finally death through syncope and collapse in from 6 weeks to 6 months.

Post Mortem.-There are no post mortem lesions found. There is a disease found in the northwestern part of this country and in Canada and Montreal called Swamp fever or Swamp disease. It has the symptoms of surra but the trypanosome has not been found in the blood nor are the lesions at post specific. Surra is very prevalent in the Philippine Islands.

Treatment.-No known therapeutic treatment has had any effect so that preventive measures are the only ones which can be counted on. Keep the horses on high land or drain the low swampy places. If the horse takes the disease this can be recognized by the peculiar symptoms. The animal will run down rapidly and in a few weeks generally dies. It is presumed that the trypanosomata are excreted in the feces and urine and these products should therefore be taken care of as far as possible or else the animal killed.

\section{INFLUENZA.}

The word influenza comes from the Latin word meaning "influence." The disease was so named in human medicine on account of the influence the planets are supposed to have upon the atmospheric conditions and the resultant changes upon the animal life on the earth. No disease spreads so rapidly as influenza. The especial cause has been attributed to several different micro-organisms. Pfeiffer isolated a bacillus which he thought was the specific cause and called it by his own name. 
Kitasato and Canon corroborated Pfeiffer's discovery. Ehrlich claimed that the germ was a streptococcus. Influenza is recognized today as a contagious disease but the means of communication are not known, probably it is through the alimentary tract. The germs may be taken into the respiratory tract first and then when expelled, lodge in the pharynx and are swallowed. It is thought that horses get the disease from eating and drinking from common receptacles. It is a good plan to have their drinking water constantly running and this will carry away any foreign matter that may accumulate. The disease is not atmospheric but it travels along the lines of commerce. The atmosphere may influence it to the extent that a decrease in ozone will produce depression while an increase in the ozone stimulates the animal.

Influenza is a blood disease and it produces catarrhal discharges from all the mucous membranes.

Semeiology.-The disease presents different symptoms according to whether it is involved with complications or not. The symptoms of the uncomplicated form of the disease are as follows: Great nervous depression and languor with high fever. During the first twenty-four hours the fever is about 106 and then it falls to 105 or 103 . The animal is inclined to lie down more or less, his legs swell, the swellings are sore under palpation, and he generally loses his appetite. After twenty-four hours there is a profuse discharge from the nose and after fortyeight hours a marked loosening of the bowels, and the mucous membranes are usually icteric. The inflammation sometimes attacks the urinary apparatus and produces profuse micturition. If the animal is not well blanketed his coat stares, breathing is accelerated and the mucous membranes are injected. The head hangs, the ears and the eyelids droop. In the corners of the eye is coagulated mucous. Often the throat is sore and there is a troublesome cough. This cough is inclined to remain with the animal and become chronic. It is thought by some that this disease produces roaring and this cannot be disputed.

In the uncomplicated case the fever generally goes down to normal in three or four days and the appetite is regained and the animal makes a good recovery in from five to ten days. The discharge subsides and the animal seems to be none the worse 

for the attack. It is thought that one attack renders the animal immune for at least six months.

The complications of influenza involve the lungs in the form of broncho-pneumonia, which is always of the contagious variety and the symptoms are those of a grave pneumonia; the symptoms being rapid exhaustion, loss of appetite and exacerbation of fever-animal dies in 5-10 days if recovery does not take place.

Diagnosis.-The diagnosis of influenza is based upon the continued fever, with great depression and symptoms of rapidly developing icteric, dark colored mucous membranes and swelling of the legs. The evidence of colic and congestion which is followed by diarrhœea indicates a complication of enteritis. The diagnosis of brain trouble is based upon the excessive violence which interrupts the otherwise continual stupor of the animal.

Prognosis.-Influenza is a serious disease principally on account of the complications. Uncomplicated influenza is only fatal in 1-5 per cent of the cases.

Treatment.-While the appetite remains the patient should have a moderate quantity of hay, good oats and bran; or even a little fresh clover, if obtainable, can be given in small quantities. The sick horse should be placed in a well-ventilated stall. To reduce the temperature give one dram of acetanilid every two to four hours. The acetanilid may be used with alcohol and strychnine. The patient must be blanketed. Never give a laxative of any sort, for the enteric mucosa is in so sensitive a state that half a pint of raw oil, or one ounce of sulphate of soda might precipitate enteritis and cause death. If founder occurs, it is difficult to treat. When recognized, hot or cold applications are useful.

\section{PURPURA HAEMORRHAGICA.}

Purpura hemorrhagica (French, anasarca) is frequently one of the sequelæ of influenza. It is an infectious disease though not contagious. There is a difference of opinion as to the specific germ of this disease. Tizzoni, in 1889, found a bacillus which he considered the specific germ. Babes corroborated it 
in 1890 and Kolb in 1891. After this Marmorek of the Pasteut Institute claimed that the streptococcus was the cause. Although the disease may follow influenza yet it may often develop and run its course as an original disease.

This disease is characterized by the destruction of the blood elements on a large scale. The blood becomes thin and oozes out through the pores into dependent parts by diapedesis; it may be circumscribed or diffused when it produces purple spots or patches (ecchymoses).

It occurs as an original disease in badly ventilated and badly drained stables or as a sequel to debilitating fevers.

Symptoms. - The fever is regular and quite persistent at 104 . The legs swell and when the swelling gets above the knees and hocks it terminates abruptly. The swelling creeps higher and higher and in three or four days the lips begin to swell and the eyelids become bloated. There is also a pendulous swelling under the abdomen. Early in the disease the mucous membranes are ecchymotic, the spots varying in size from $1 / 4$ to $1 / 2$ inch in diameter. They may be seen on all the mucous membranes.

In some severe cases there is a fetid discharge from the nose. The mouth fills with a ropy saliva. A yellowish, gummy liquid comes out through the skin on the legs and trickles down the hair. The swelling is so great as to prevent the horse from eating and it diminishes the caliber of the air passage and causes wheezy breathing.

When the symptoms develop as above described the horse usually dies within 5-15 days. The five-day course accompanies the disease of an original lesion; the fifteen days course is the result of some other previous disease. The kidneys are usually inactive sometimes to the extent of complete ischuria, and toward the latter end of the disease the horse may have bloody diarrhœea and colics. Sometimes more or less extensive sloughing of the skin takes place. This is an indication of a very severe case.

Post Mortem.-Upon opening the body we find ecchymotic extravasations everywhere, especially on the mucous membranes. In addition there is more or less general infiltration. All parts are œdematous. The large bowels will show quite a liquid con- 

dition of the contents, which are bloody. The blood itself is thin and coagulates very slowly.

Treatment.-The serum treatnent would be the best for its action upon the blood would kill the germs. Marmorek man"1factured a serum which has been used with good results in some cases and in others not. Use antiseptics and stimulants internally. Bichromate of potassium in one to three grain doses in one or two ounces of water given 2-3 times a day will tend to destroy the germs in the blood. In connection with this use tincture of iron and sweet spirits nitre in liberal doses. Some add a little quinine. The prescription is as follows:

$\mathbf{R}_{\mathbf{k}}$

Tr. Ferri. Chlo................. ounce ij

Quinini Sulph.................. dram iv

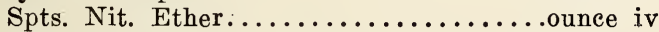

Aqua q. s......................pint $j$

M. Sig.-Give 2 ounces every 4 hours in a moderate case, but if the case is bad give it every 2 hours.

In addition to this give the bichromate. Do not give a febrifuge.

The iron has double action as it helps make the red blood corpuscles regenerate and tends to limit the hemorrhage by its astringent action upon the blood vessels.

If the muzzle begins to swell bathe it with warm water. In a bad case keep a man at this treatment all the time, night and day. The warm water drives the œdema to some other part where it is not so dangerous. Never tap the swellings as sloughing will follow. The fluid will not flow out. In case the dyspnœa interferes with the respiration perform tracheotomy, but an operation upon a horse with purpura is apt to be dangerous and fatal. Keep the horse quiet and feed him generously with oats, corn, bran, etc. All mild cases recover, but $40-60$ per cent of the cases are fatal.

\section{SCARLATINA.}

Scarlatina is a very infectious disease, closely related to purpura, but it has some prominent differentiating features. Some have thought it a milder form of purpura but they have overlooked important features. Scarlatina comes on oftener as an 
original disease than purpura. In scarlatina the hemorrhage is in the form of petechix and the blood spots are scarlet, while in purpura they are purple. In scarlatina there is always some sore throat, in purpura never. The swellings in scarlatina are usually in patches and lumps usually on the trunk, while in purpura the legs swell and also the abdomen. The swellings are smooth while in scarlatina they are lumpy. In scarlatina the lymphatic glands suppurate, while in purpura they never do. In both diseases the fever and pulse are about the same. Animals usually recover from scarlatina while in purpura many die.

Treatment.-A case of scarlatina is apt to hang on for two or three weeks without any change, while a case of purpura gets better or worse within a week. In scarlatina we have to treat the sore throat. Put counter-irritants and bandages on the throat and use such antiseptics as guaiacol. Give quinine in larger doses than for purpura. Give iron gargle for the throat and keep the horse mildly stimulated with nitrous ether. In some bad cases the throat ulcerates and then it is a good plan to swab the throat with nitrate of silver. Use the swab on the end of a long stick.

Scarlatina in the horse seems to conform with scarlet fever in the human but purpura has no similarity whatever. So far as the relationship between scarlatina and purpura is concerned it is found that scarlatina runs into purpura before death, but this does not necessarily mean that the two diseases are the same. It is seldom that animals die from scarlatina.

Post Mortem.- In scarlatina there is an absence of the general infiltration that is seen in purpura. There is difference enough to warrant us in saying that the two diseases are not the same.

\section{INFECTIOUS ANEMTA.}

This disease, known also by other names, such as Pernicious Anemia, Swamp Fever, Mountain Fever, American Surra, Malarial Fever, Typhoid Fever of Horses, the Unknown Disease, No Name Disease and Plains Paralysis, is more or less prevalent in Texas, Nevada, Kansas, Nebraska, Colorado, Wyoming, Mon- 

tana, North and South Dakota, Illinois, Iowa, Manitoba and several countries in Europe.

It has been under investigation for the last three years by Drs. John R. Mohler, M. Francis, R. P. Marsteller, Winfred B. Mack, A. T. Kinsley, L. Van Es, and others.

It is a distinctly infectious disease affecting horses, mules and asses. It used to be supposed to be confined to swampy regions, but within the last three years has been found in altitudes as great as 7,500 feet.

Etiology.-The specific virus has not yet been isolated, but it is known to be specific by inoculation with the blood and blood serum producing the disease, even after the serum has been filtered through a fine Pasteur 'ilter, consequently the virus must be infinitesimally small, or a toxine.

Dr. Van Es has recently, 1911, found that the urine and feces contain the virus, and that horses eating the litter soiled by horses with this disease develop it. The Pasteur filter would remove all known bacteria and protozoa.

Semeiology.-It is characterized by progressive anemia and remittent fever. The horses become dull, listless, weak and finally stagger; the temperature may run as high as 106 for a few days, then gradually fall to 102 and stay around that point for two or three weeks, and then rise again for a week; the pulse becomes rapid, small, hard and thready, and jugular pulse is seen after a few weeks. The mucus membranes gradually become paler and paler; sometimes they develop a yellowish or even a mahogany color in some cases. They also occasionally present hemorrhage spots (petechiæ). Oedematous enlargements on the under side of the belly, sheath, mammæ and legs. There are profound changes in the blood. The red corpuscles from a normal of seven million per cubic millimeter sometimes drop as low as two millions. There is little or no change in the number of white corpuscles. The blood becomes thin and watery. The appetite usually remains good throughout the course of the disease.

Post Mortem.-Great emaciation and loss of fat are seen, all parts are extremely pale. Local hemorrhages may be seen, especially in and around the heart, which is generally enlarged. The lungs are studded with petechiæ, and there is besides a little 
serum in the plural cavity, also in the pericardium. The liver is usually sound, with the exception of possibly slight areas of degeneration. The spleen is somewhat enlarged and covered with petechiæ. The kidneys are anemic and flaccid and usually show, microscopically, a chronic parenchymatous degeneration. The lymph glands are usually enlarged and hemorrhagic.

Prognosis.-Prognosis is very unfavorable. They practically all die, although a few, perhaps $15 \%$ or $20 \%$, may yield more or less to treatment, and live along for two or three years, but finally succumb. No horse was ever known to fully recover.

Treatment.-The treatment is very disappointing, but systemic treatment would be as follows: In the earlier stages reduce the fever with actetanilid and cold sponge baths. Stimulants, such as nux vomica and alcohol, are indicated for the pulse. For the blood give tincture of iron and Fowler's solution of arsenic in liberal doses. In all probability the arsenic is the only remedy that has much, if any, effect. Isolate the affected horse from the healthy ones and burn all of his litter and disinfect the stable daily with a $5 \%$ solution of U. S. P. Liquor Cresolis, or any other coal tar emulsion, and sprinkle the floor occasionally with air slacked lime, or chloride of lime. Investigations are in progress with a view of producing a vaccine or serum that will protect the healthy horses where the disease is prevalent.

\section{RHEUMATISM.}

Rheumatism is a non-contagious disease that arises in the body and it is not specific. It is a very peculiar constitutional disease. Its real character is not known, but old theories attribute it to an excess of some natural acid in the blood, such as lactic acid, uric acid, etc. This is evidence of indigestion and in every case of rheumatism we can trace the condition back to some form of stomach disturbance. In herbivora we have more hippuric acid than in other animals and if this runs to excess we are apt to get rheumatism.

Rheumatism is divided into various classes according to the part affected. For instance, we have rheumatic fever. In such a case the whole system is affected and there is no localization of 

the affection. It is generally fatal if the animal is not very strong. Death comes either from endocarditis or from valvulitis or both. Occasionally death is due to pericarditis. Another form of the disease is found in the localization of the trouble in the loin and it is then called lumbago. When it attacks the nerves it is called neuralgia. The nerve usually affected is the trifacial nerve. If the disease locates in the muscles it is called muscular rheumatism. Sometimes it attacks the sciatic nerve and then it is sciatica. When it attacks the joints it is called articular rheumatism. The disease is inclined to attack the synoval membranes of the joints and tendons and it frequently jumps about from one place to another and is called flying rheumatism or metastatic rheumatism. It then seems to leave the part of the body first affected.

Rheumatism may be either acute or chronic.

There are some who do not consider rheumatism to be a specific disease, but claim that it is only a shield for ignorance. Sir Richard Christison found that by injecting a dog with lactic acid he could produce rheumatism. Many are now looking for the specific germ of rheumatism. The disease is, probably, largely hereditary. The exciting cause may be due to faulty assimilation as is seen in dogs fed on meat only. In some cases it occurs without any apparent exciting cause but there is usually exposure to cold and dampness. Animals that are left out in bad weather are apt to get rheumatism.

Semeiology.-Rheumatic fever usually starts with a temperature of 104 or more but sometimes not over 103. A rheumatic animal is more or less stiff and not inclined to move. The appetite is good and does not vary much. The animal may or may not lie down. The pulse is increased in frequency and hardness. It runs about 45 to 60 .

The fever can be reduced with acetanilid but as soon as the drug is stopped the fever will come back again. If a little of the blood is drawn it will coagulate very quickly. After the disease has been running for ten days or a few weeks the animal may die suddenly. Or if he lingers along he loses flesh. His legs swell and you get a jugular pulse. He may live in this condition for several weeks. 
Post Mortem.-Post mortem shows that the trouble is in the heart, which may give evidence of endocarditis, valvulitis or pericarditis. In all cases the animal is liable to die suddenly from syncope. In inflammatory rheumatism the joints swell but never run to suppuration unless bruised. The parts are sore and produce great lameness. If left to run its own course the inflammation is apt to run five or six weeks. It usually leaves the joints permanently enlarged, especially if in the hock. Metastatic or flying rheumatism is inclined to attack the sheaths of the tendons in the legs. The disease migrates from one leg to another but is usually constant. The horse never recovers from rheumatic arthritis after ossification of the exudates takes place ard has what is called chronic rheumatism.

Sciatica comes on suddenly. The horse steps short with the affected leg and does not want to be backed up; the act of backing up seems to cause muscular pressure and it consequently causes pain. If he is made to back up he will carry the leg that is affected with rheumatism. Upon manipulating the sciatic region you can detect the soreness. In neuralgia rheumatism attacks the fifth nerve. The horse will hold his head tipped more or less and there is twitching of the facial muscles and partial closing of the eye. The horse is disinclined to move and shows many evidences of distress. Pressure upon the part will cause him to wince. Neuralgia is not very common in the horse.

Lumbago.-When this condition attacks a horse he will show stiffness, will turn with difficulty and will not move readily. His back will be arched. In bad cases he is likely to lie down and then is unable to rise, perhaps for a week or two.

Semeiology.-The symptoms of lumbago are well marked in the dog. The subacute form sometimes attacks the intercostal muscles and is called pleurodymia. It closely resembles pleurisy but in pleurodynia there is no fever and auscultation reveals no friction sound. It resembles pleurisy in its fixed condition of the ribs, soreness of the intercostal muscles, careful breathing, turning out of the elbows, etc.

A subacute attack in the muscles of the neck seems to cause contraction of the neck muscles so that the head is turned to 

one side. On the concave side of the neck the muscles stand out prominent and are called the cords of "torti colis."

In muscular rheumatism the horse has a stiff action and is dicinclined to move. He has probably been exposed to cold drafts and consequently caught cold in his muscles.

Rheumatism is not fatal unless it attacks the heart, which it does in two forms. When the spasmodic pain attacks the heart it is called angina pectoris. In the human there is the sensation of suffocation. The presumption is that if the heart is interfered with it may produce death by spasm of the heart but this cannot be detected in the horse.

Acute rheumatism in the form of rheumatic fever is never fatal unless it attacks the heart. In this case only a few cases recover.

Subacute or Chronic.-From what is known we find that this form of rheumatism generally attacks older animals. When it attacks older animals it is more mild. The attacks are painful, but not dangerous. The synovial membranes may become affected but when the disease locates in the muscles it often becomes chronic. In old horses it may cause sudden and severe lameness which may leave in a few minutes, or it may last for hours. In dissecting a case like this you will find calcareous deposits in the synovial fringes. It is supposed that this calcified fringe gets in between the two bearing surfaces and causes the lameness. If the horse is compelled to proceed the deposit is pushed back in place and the lameness ceases.

Torti colis may be caused by violence and rupture of the ligamentous attachment to cervical vertebra on one side of the neck and this makes the head turn in the opposite direction. In the very young animals this can be cured by means of splints on the neck. This form of torti colis is not rheumatic in its nature.

Treatment of the Acute.-In rheumatism there is an unhealthy condition of the blood. Therefore antiseptics are indicated with the general idea of increasing the alkalinity of the blood and of changing the urine from acid to alkaline. Particular attention should be paid to the digestion. Salines are effectual. Salicylate of soda should be given in liberal doses. 
Give three dram doses every hour night and day for ten to fifteen days in order to get the desired effect. The best treatment known is salicylate alkaloid of oil of wintergreen (aspirin), but this is very expensive. The human dose is 'five grains every four hours.

Other salines are nitrate of potash, one dram three times a day, sulphate of magnesium and all of the laxative salts, iodide of potash, vegetable alteratives, etc.

The following is good treatment:

$\mathrm{R}$

Sodii Salicyl .................... ounce vi

Fl. Ex. Colchici .................... ounce ij

Aqua qs. ad.......................... pint $i j$

M. Sig.-2 ounces every 4 hours night and day.

The first remedy you adopt should be used for about ten days and then switch to some other. An acute case seems to run a course of about six weeks. If you have been using the prescription given above then change to sulphate of magnesium.

It is useless to apply stimulating liniments. An application of chloroform for a few minutes, which is allowed to evaporate, will allay the pain. The following is a good liniment:

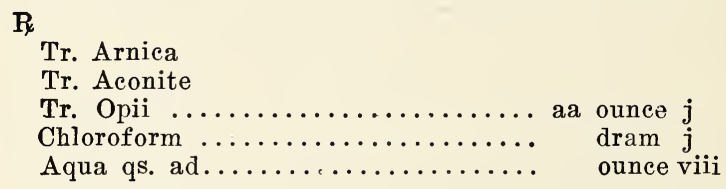

In all acute cases of rheumatic lameness and swelling a fly blister is very beneficial.

\section{LYMPHANGITIS.}

This is an inflammation of the lymphatic ducts. Lymphadenitis is inflammation of the lymphatic glands. There are four forms of lymphangitis, plethoric, anæmic, mycotic or epizootic and septic. The acute plethoric form, develops suddenly in horses that are plethoric and working every day, but laid off for a holiday or a Sunday. The plethoric condition of the horse while at rest is the etiological factor in the case. It may attack any part of the body but generally one of the hind legs. It is usually found in the morning after a day of rest, such as Mon- 

day, for instance. This disease is called by some Monday morning sickness. In Scotland it is called the "weed." In England it is called the "shot of grease" or the "shakes." The affected lymphatics swell and become inflamed.

Pathogenesis.-The blood is plethoric and the lymphatic ducts become congested on account of overwork. We presume that in such a case the metabolism during the forty-eight hours previous to the attack has been slight and the nutrition great enough to clog the lymphatic ducts. This seems to act much the same as active congestion in altering the nutrition of the part. The inflammation may be so severe as to run into the third stage and may result in an abscess.

Symptoms.-If the horse is in a warm stable he will be found in the morning with a chill, which is sometimes very severe. The severity of the attack depends upon the severity of this chill. In the course of a couple of hours the doctor will be called and when he arrives he will find the horse lame in a hind leg. The temperature will be about 106 . The swelling increases rapidly and runs down the inside of the leg in the inguinal chain of lymphatics and varies in size from the size of a linger to that of a wrist. By the end of the first day the whole leg becomes involved from the foot to the stifle. When the inflammation subsides it will leave the leg large for at least ten days and sometimes permanently. An abscess may form as large as a fist or it may hold a quart or two of pus. During all this time the animal is off his feed. His appetite returns as the fever subsides. One attack predisposes an animal to another, and each time the affected leg is left a little larger until the horse gets the chronic big leg, called Elephantiasis. This enlargement is fibroid all the way up the leg and it is incurable. If the enlargement is not fibroid in nature then being œdematous, exercise will help drive it out.

Treatment.-If called to an acute case during the chill, treat it vigorously. Give aconite and alcohol or spirits of nitrous ether every fifteen minutes until the horse begins to sweat and keep him well blanketed. Drop off the frequency of the dose as soon as the chill subsides. Give him aconite, spirits of nitrous ether, nitrate of potash and water after the chill has subsided. Give these every hour or two and take off some of the blankets. 
In addition to this give a full purgative dose of aloes and dose with acetanilid every hour for four hours. The dose should be governed by the weight of the horse and the height of the fever. Keep up the fever mixture until diuresis is produced and then drop off to every four hours and then to three times a day. As soon as the treatment has been started, have a man bathe the leg of the animal with warm water. Take care that the water is not too hot, for it will scald the leg. The water should be 115 degrees. The bathing should be done three times a day, about an hour at each time. It is very important to have the affected leg warm and well wrapped. If this treatment is persisted in the swelling will go down and the fever will subside. Continue until the leg is reduced one-half. The animal should not be fed for forty-eight hours. After the leg begins to get better, exercise should begin. Walk him around in a warm place, each time increasing the exercise, commencing with about ten minutes. If the case comes to you neglected, then the treatment is different. Give him purgatives but do not bathe. Leave out the ether and acetanilid. Give him potash and exercise him. If the swelling can be driven out the case is curable, otherwise not.

Stallions and jacks have sores in the form of eczema which come from lymphangitis. The sore starts with a small pimple which itches. The horse either bites or scratches the place until it becomes raw and granulates and has a tendency to spread. The treatment consists in removing the cause. If feeding is the cause, lessen it and substitute bran. Give a mild purgative. A large stallion should not get more than seven drams for a purgative dose. Stallions cannot stand as much as a working horse. Give a purgative once a month and between the purgations give sulphate of sodium night and morning. For local treatment mild applications are the best. If much granulation tissue forms around the sore it must be removed with the knife and then apply the white lotion three or four times a day. Rub the sore gently to remove the loose scabs. Jack sores are more difficult to heal. 



\section{EPIZOOTIC LYMPHANGITIS.}

This is a new disease in some parts of the world and old in others. It is caused by a fungus called the saccharomyces farciminosus. The disease is also called ulcerative or mycotic lymphangitis. It is communicable to other horses by contact with anything that carries the fungus.

The fungus can grow on the horse and mule. After it enters the wound it begins to grow slowly and soon starts up an inflammation (in three weeks to three months) and a nodule forms under the skin with hardening and thickening of the surrounding lymphatic ducts. The nodule softens, ruptures and discharges pus and an indolent ulcer forms. It usually occurs on one leg, may be on two and it may occur on any part of the body. The poison is carried from this point and it may break out anywhere. When it attacks the leg it involves the whole leg from the foot to the body. A few cases recover spontaneously and many are curable by proper treatment if applied early. The rest continue to grow worse. Inasmuch as the pus carries the germ the disease is liable to spread to other animals. It is fatal in ten to fifteen per cent of cases. The pus is yellow or grayish yellow and is often mixed with blood. Sometimes it contains flakes.

The first symptom is an indolent sore covered with pus and a scab surrounded by a slightly swollen area with radiating lines running from it. The ulcers may heal slowly and recur and the skin becomes thickened. The affected horse does not show any constitutional disturbance unless the case is very advanced. This disease may be mistaken for farcy. In farcy the mallein test is positive, and the pus is oily or glutinous and the ulcers are cupshaped. In lymphangitis the mallein test is negative, likewise the Strauss test and the agglutination test, the pus is thick and creamy, the ulcers are flat and show more granulations, and the nodules are less firmly attached to the skin and more movable than in farcy. There is an absence of the bacillus malleus and the presence of the specific fungus, the saccharomyces farciminosus.

Treatment.-If the case is gotten early enough remove all the tissue involved with the knife. If removal is impracticable open 
the abscesses and treat antiseptically. Bichloride 1-250 or Lugol's solution or a solution of formaldehyde 1-200 are appropriate antiseptics.

All advanced cases should be destroyed.

Anaemic lymphangitis is not seen in the horse. Septic lymphangitis is seen as a result of sepsis in wounds of any kind. The parts around the wound swell, become painful and the wound suppurates, and sometimes small abscesses form in the ducts leading from the wound.

Treatment.-After opening the abscesses, local antiseptic wet packs continuously, applied, with thorough antiseptic treatment of the original wound, and stimulants internally will control it.

\section{AZOTURIA.}

Azoturia is a non-contagious blood disease of the plethoric type. It is almost if not entirely peculiar to horses. It may exist in the human. The disease is characterized by sudden prostration and if the animal is down he is usually unable to rise. Azoturia invariably follows exercise after a period of enforced idleness and continued high feeding on such food as oats. In such a case an excess of nitrogen is probably developed in the system and the excretory organs cannot take care of all the extra waste material. It occurs in all ages and breeds, in both sexes. We find it more common in half bred draft horses than in full blooded animals, probably because the half bred is more common, more numerous.

Etiology.-Azoturia is due to idleness, high feeding and then exercise. The resulting condition is one of plethora. The animal sometimes gets down and becomes cast in some way or other and in his efforts to get up he exercises so violently as to bring on the disease. In case of indigestion from idleness if colic pains arise, the struggles of the animal may bring on an attack of azoturia. If a horse is to be cast for an operation and has not been prepared by dieting, his struggles in the apparatus may bring on an attack of azoturia. If a highly fed animal is idle he will become plethoric. All of the organs become sluggish in action and the blood becomes rich in solids at the expense of the 
. 

watery constituents. The animal becomes indolent and his circulation is slowed. When he begins to exercise again the circulation increases, the pulse in some cases running up to 60; he feels good and takes very active exercise, and as a result of this exercise there is tissue metamorphosis. The waste material produced in the system is nitrogen. Every contraction of the muscles separates the nitrogen from the tissues. The muscles get their nitrogen from certain kinds of focd. The surplus nitrogen must be eliminated from the system by the kidneys or it will poison the blood. The kidneys excrete it in the form of urea. The surplus water and salts in the blood of a plethoric animal can be gotten rid of through other channels but the kidneys must take care of the excess of nitrogen. The thick blood cannot circulate so freely through the organs and we get a capillary stasis, the kidneys become congested and there is an impaction of the capillaries with blood. This interferes with the action of the kidneys and the coloring matter of the blood is forced out into the urine, giving it the dark color which we see in the urine of azoturia.

The action of the kidneys may be so disturbed that there is total suppression of the urine and the horse may die in two hours; but if the kidneys are partially active then the life of the animal is prolonged. When he recovers, it is because the kidneys are restored to their normal activity, but if he dies it is because the kidneys cannot eliminate the urea and uræmic poisoning takes place. Uræmic blood poisoning is the immediate cause of death. Other organs are congested as well as the kidneys, the lungs especially. As the animal goes down he gives way to a peculiar motor paralysis of the muscles, due to muscular congestion. This congestion causes pressure upon the trunk nerves and the muscles become paralyzed. The muscles most often affected are the giuteal, posterior portion of ihe dorsal and the anterior crural region. The congestion of these muscles is often so severe as to interrupt the circulation and a cessation of the muscle activity takes place, leading to atrophy. When the congestion is very tense with little swelling, the muscles get hard and the pressure upon the nerves is severe and often fatal. Usually, but not always, the hind parts are affected, more 
often the right hind quarter. A rather plausible theory has recently been advanced that the disease is due to autointoxication by the product of fermentation of the mass of food in the colon. The disease develops only in heavy feeders and during idleness the bowels get torpid, giving the ingesta ample opportunity to ferment, producing some subtle poison that does the mischief.

Post Mortcm.-Where death sets in soon after congestion, we shall find the muscles, particularly the affected ones, darker colored than normal. The heart is filled with black clots of blood; the kidney and liver are soft and the kidneys may be gangrenous. There may be abscesses in the kidneys. The cerebral meninges will be more or less congested with some effusion in the arachnoid space. The animal will be fat on the inside even if lean on the outside. If the blood is plethoric, the animal does not need to be fat in order to be predisposed to this disease. Animals that are hard worked usually get an abundance of oats and they are the ones most apt to take the disease though the work may keep down the fat.

Semeiology.-When the horse is taken out after a period of idleness he feels good and dances around full of life. The distance he will then be able to go depends upon the amount of nitrogen set free by the muscular exertion. He may go onefourth of a mile, he may go twenty miles, but he soon begins to sweat and blow. The blowing is due to congestion of the lungs, the sweating to congestion of the skin. He begins to look around, has an anxious countenance and gets lame in the hind quarter. The fetlock knuckles-that is the first symptom of the paralysis. The affected leg drags, he cannot bear any weight on $\mathrm{i}$, then the other fetlock knuckles and that leg becomes helpless and all at once the horse is down. During this time the muscles of the back often swell hard as a board. The horse lies quiet for a time but colicky pains come on, he gets restless, the nerves become intensely excited, pulse tumultuous, weak, uneven, and finally dicrotic, and there is a convulsive motion of the limb. In the early stage you will not find any abnormal condition of the urine, but within an hour or two you will find it thick, and a dark coffee color. The color is due to the pigment set free from the blood; the thickness is due to mucous. Sometimes it is so 

thick that it will not flow through the catheter, and you have to assist it by putting the hand in the rectum and giving the bladder gentle pressure. The fever rises to 103 or 104 . Pain and nervousness cause him to make attempts to get up and he can get up upon his fore feet but his hind parts are helpless. He may walk on his front feet and drag his hind parts along for some distance.

As the disease progresses and uræmic poisoning sets in the animal becomes delirious (the first effect of uræmia) and runs on to a comatose condition and finally death. Death usually occurs in convulsions. The mortality in the city is about 60 or 70 per cent. Death occurs in about 48 to 72 hours, though the disease often runs on to five or six weeks. Sometimes the urine contains tube casts, hæmatin and hæmoglobin. The tube casts indicate nephritis and in case of abscess there will be pus in it. In case of gangrene gas will bubble out through the catheter.

In mild cases the symptoms are only partially developed. He may go down and get up again but there will be pain and lameness in one quarter, in that respect different from colic. The animal often gets well after lying down an hour or two. It seems that a three days' stay in the stable is worse than a three weeks' period of idleness, for in the longer time of rest the animal gradually loses the good appetite he had at hirst and consequently eats less and the system becomes used to the change. The capillaries become enlarged and can accommodate the larger amount of blood.

Traatment.-Wherever you find the animal, draw off the urine to relieve the distress of an overfilled bladder. If he is nervous give him an ounce of fluid extract Gelsemium for the first dose and if a second is needed, a half ounce. It is doubtful if a purgative is needed, as there is already a lack of water in the system and a purgative would further reduce it. I never found much benefit in purgatives, though I usually give an average dose of aloes. Diuretics are dangerous; if the congestion is severe the diuretic will surely kill. The potassium diuretics are especially dangerous. Give a mild stimulant. 


\section{$\mathbf{R}$}

Fl. Ex. Gelsemium ..................ounces ij

Potassium Bromide .................... ounces ij

Aqua qs. ad....................... pint i

M. Sig.-Give a 2-ounce dose about every three or four hours.

In addition to this Dr. Ellingwood suggests lithium citrate (1 dram in water) every two or three hours for the first 50 or 60 hours, then three times a day.

For local treatment use hot fomentations. Wring out a rug in hot water and apply over the loins. Cover with water proof and then dry blankets. This keeps the steam in. During convalescence give nux vomica. Remember that a horse's chances are much better for recovery if he is able to remain in the standing position. If he can bear about two-thirds of his own weight slings may be tried, but they usually aggravate the symptoms.

If the muscles waste in the hind quarter, treat by stimulating with setons. Put in three setons about two inches apart and leave them in about six weeks. Send the animal to pasture and give a good long rest. It may take six months for the muscles to redevelop. If necessary you can medicate the setons with cantharides, turpentine, etc. Be sure to give gentle exercise, but give all he can take. If the wasting is extensive and severe get him up for exercise every two or three hours.

Although the urine is so dark colored and full of the coloring matters of the blood, yet a microscopical examination of a portion of it under the high power lens does not show any pigment crystals. Also an examination of the blood does not reveal any diagnostic features. The red blood corpuscles are normal in color and size and do not give evidence of having lost their constituents. In fact some authorities consider the pigment body which colors the urine to be directly a muscle element and not a blood. Muscle hæmoglobin, however, has the same composition as blood hæmoglobin.

Friedberger and Froehner say that the blood in azoturia will show the hæmoglobin crystals.

Azoturia is a complex affection. It is rather a disease of the liver and blood forming functions than of the kidneys, although the vulgar idea is that the disorder is one of the urinary tract. 

It is directly connected with a plethora in the blood of nitrogenized constituents with extreme nervous and muscular disorder. The poisoning is seemingly not present when the animal is taken from the stable, yet some cases develop a fatal attack while being harnessed and hooked up, before leaving the wagon floor. It would seem as if the aspiratory power of the chest under the sudden exertion of the over-fed animal speedily drew from the liver and abdominal veins (portal system) the accumulated store of nitrogenous matter in an imperfectly oxidized or elaborated condition, and as if the blood surcharged with these materials was unable to maintain the healthy functions of the nerves and muscles. The pathology, however, is little understood. We must content ourselves for the present with theories.

\section{DISEASES OF THE CIRCULATORY SYSTEM.}

There are three means of determining the diseases of the heart and blood vessels;-inspection, palpation and auscultation. By inspection you detect any change in the position of the heart and the force of the heart beat can be determined. The character and rhythm of the pulse you get by palpation. Auscultation reveals the sounds of the heart. The heart has a normal sound and any deviation from this can be detected by auscultation.

The various phenomena of the heart diseases are (1) Languor; (2) Impeded respiration during the exercise, often amounting to distressing dyspnœa; and (3) Condition of the visible mucous membranes.

The diseases of the heart are divided into two classes,--functional and organic. The functional are acute and are three in number, palpitation (thumping), cyanosis (blue disease) and syncope (faiting fit). Spasm of the diaphragm, sometimes spoken of as thumps, is often mistaken for palpitation. The organic diseases of the heart are chronic or structural diseases.

\section{PALPITATION AND SINGULTUS.}

Palpitation (thumps) is a tumultuous action of the heart. It is a disturbance of the rhythmic action of the heart and is some- 
times seen as a symptom of some other disease, for instance anæmia. Hard work often brings it on, especially on a hot day. It occurs through a disturbance of the equilibrium of the nervous system (sympathetic). When the condition comes through exertion, the animal is exhausted and the heart is over-taxed. The action of the nerves may be direct or reflex. Acute indigestion may cause thumps. This form of the disease may come on while the animal is standing quietly in the stable.

Singultus or spasm of the diaphragm (hiccough) is caused by excitation of the phrenic nerve center and it is thought that this excitement is due to some form of indigestion. Too hard or too fast work after a full meal is apt to cause hiccough and it may be mistaken for palpitation. It may sometimes develop without any apparent cause. The condition is seen most frequently in fast horses. If a horse comes in from a run with hiccough and in great distress, he may recover in twenty minutes, but if it is thumps he may be laid up for several days.

Semeiology.-There is increased effort with decreased power of the heart. The pulse is irregular in every way. It is generally very rapid, say at seventy-five, but it is up and down and the force of the beat is irregular. The result is passive congestion of the lungs and there is rapid breathing with considerable dyspnœa in a bad case. No signs of pain especially, but the distress of the animal is great. There is a jugular pulse, distension of the veins of the head and neck and the palpitation of the organ itself is sometimes so great as to shake the whole body. In hiccough there is sudden contraction of the diaphragm, in spasms, which also gives an impulse to the body, but you cannot see the pounding of the heart on the ribs. Hiccough is not as frequent as palpitation. You may get several hiccoughs in a minute and on the other hand only one in the course of three minutes. In palpitation there is apt to be two or three a second. Hiccough may stop any time, while in palpitation the thumps keeps up for one to two hours to a day or two. In palpitation the blood vessels will be greatly dilated, while in hiccough they will not.

Treatment.-In all cases there should be perfect quiet. If the palpitation is due to organic disturbance of the heart's action 


$$
\text { , }
$$



then strong stimulants are indicated, such as alcohol, whisky, digitalis (most important of all) and nitro-glycerine is often resorted to when the others fail. Tincture of cactus is good in the human when the palpitation is due to indigestion. If the palpitation is due to the disturbance of some other organ then the condition is reflex and it is necessary to treat the organ disturbed. In case of anæmia treat the blood; in plethora and too much fat, give a purgative, reduce the feed and give mo ie exercise. The excited condition of the heart should also be treated. For this aconite is the best for it slows the heart without depressing it. Give about ten drops of the tincture of aconite or about seven drops of the extract. Give whisky also, a half pint for the first dose and then, if necessary, repeat in two ounce doses every fifteen minutes until the animal is relieved. After the attack is over then remove the cause. If the horse is suffering from nervous exhaustion then give cardiac stimulants, such as digitalis. Powdered digitalis and solid extract of belladonna are prescribed, twenty grains each, night and morning. If the blood is faulty give iron.

Treatment of singultus depends upon its severity and duration. It usually disappears as the horse cools and after drinking a few swallows of cold water. If not give half a grain of strychnia hypodermatically. If this does not stop it give four grains of morphia in the same way.

\section{CYANOSIS.}

Cyanosis is known as the blue disease. This occurs only in young animals,- - those newly born. It is sometimes found in foals immediately after birth and is due to the non-closure of the foramen ovale between the two auricles. This allows a mixture of the venous with the arterial blood in the left cavities of the heart. It is characterized by a dark purple or bluish color of the visible mucous membranes, shortness of breath and general coldness and feebleness. Foals thus affected generally live only a few hours. If the young animal should live it would grow up very weak. This condition is often seen in babies-blue baby it is called-and they may recover by good nursing. If they can be kept alive for eight or ten days they will get well. 


\section{SYNCOPE.}

Syncope is fainting and when applied to the heart it means a fainting fit. The heart fails and loses its irritability and will not respond to the nerves. This causes the nerve centers to be anæmic and a consequent lack of blood to the brain. It rarely occurs among horses. The condition could be induced by a rapid and great loss of blood or by intense pain or by mechanical interference with the circulation of the brain.

Uterine hemorrhage is a common cause of syncope in the human. Rapid bleeding from the jugular produces it. You should be able to differentiate between syncope and apoplexy. Apoplexy is caused by hemorrhage into the brain while syncope is caused by a lack of blood, or anæmia of the brain. In apoplexy there is a purple appearance; in syncope pallor.

Semeiology.-There may be a partial or total loss of consciousness; the pulse sinks rapidly and the animal drops to the ground. The surface of the body turns cold, the breathing is scarcely to be perceived, but the state lasts usually only a few minutes. If the case is attended with much hemorrhage it may be fatal. In paralysis of the heart the symptoms may be similar to those of fainting. In apoplexy there is stertorous breathing.

Treatment.-Dash cold water on the head. Lay the animal flat with his head as low as possible in order that the blood may flow into the brain with the least resistance. Do not let the animal get up too soon or else the attack may return. As soon as the consciousness returns give whisky or carbonate of ammonia. Then afterwards general tonics, rest, and nourishing foods are indicated. Remove the cause.

\section{ACUTE INFLAMMATORY DISEASES.}

This will embrace myocarditis, endocarditis and pericarditis, Pericarditis is an inflammation of the pericardium, the serous sack surrounding the heart; endocarditis is the inflammation of the serous lining of the heart, the endocardium; myocarditis is the inflammation of the muscle.

Pericarditis runs about the same course as pleurisy (acute, 


with which it is often associated) and terminates in the same way with serous effusion into the pericardium, constituting hydropericardium, the same as hydrothorax.

Etiology.-Pericarditis may be induced by cold, damp stabling, exposure and fatigue, wounds caused by broken ribs and blood contamination. Diseases that usually exist as complications are influenza, strangles, purpura hemorrhagica, pyæmia, rheumatism and probably most common of all is rheumatic fever. These diseases may cause the inflammation of the pericardium through the blood, but the inflammation can also extend to the heart sac by the inflamed adjacent tissues.

Semeiology.-When arising as it usually does from some previously existing fever, the symptoms of the original disease will be the most prominent. As the pericarditis develops you will notice that the heart gets weaker and becomes irregular in rhythm and strength; the heart then becomes so weak as to bring on distressing dyspnœa, such as seen in influenza and rheumatic fever. If the inflammation is not severe the effusion that follows will be small. The various stages will be like those of pleurisy,first, congestion; second, inflammation; third, effusion. In case of extensive effusion the fluid often coagulates and coats the heart and the inside of the pericardium, the layer may be a half inch thick. This causes the muscles of the heart to soften and weaken. The pulse gets weak, soft and fluttering and there is always considerable fever usually about $104^{\circ} \mathrm{F}$. This condition tends to dyspnœa and consequently a watery effusion increases in the sack. The dyspnœa is due entirely to the faulty thoracic circulation. In consequence of the altered circulation and passive congestion there is more or less exudation and the lungs become sufficiently involved to get a cough associated with the disease. With the increased weakness of the heart there will be coldness of the extremities, œdematous enlargements, loss of strength, finally collapse and death. Even where the trouble is moderately severe it usually leaves a chronic lesion and affects the horse afterward. Ante mortem clots may form in the posterior aorta and cause sudden death.

Treatment.-If the condition can be diagnosed in the first stage depletion is indicated. You can give diuretics and quinine. 
Then after the first stage is passed stimulants are indicated, mild at first but increased as the weakness develops. If you let the heart get weak then the effusion will be greater, so you must keep up the heart. The heart in this condition is very susceptible and digitalis cannot be used in a mixture but must be given once every three hours alone, as the case requires. Counterirritants to the left chest or hot fomentations are good for the heat allays the pain; cold will aggravate it. In the third stage cantharides to the left side can be applied as a vesicant. Give iron and stimulants to maintain a strong heart. Salol and quinine are also indicated. The salol is usually given in dram doses every three to six hours. Acetanilid can be given with it.

But in spite of all you can do the animal as a rule dies and the autopsy shows serum in the pericardial sack and accumulations of coagula around the heart. Following some other disease, as it often does, treat the original condition as its nature seems to indicate.

\section{ENDOCARDITIS.}

This is inflammation of the endocardial lining of the heart. Associated with it is valvulitis, inflammation of the valves of the heart. Endocarditis frequently occurs as a complication of rheumatism. It is a more frequent disease of horses than is generally known and often gives rise to symptoms that are first obscure and unnoticed. In influenza we 'find the heart becoming involved in consequence of the morbid material conveyed through the heart by the blood stream. Septic diseases in any form may produce their evil effects upon the heart through the channel of the blood.

In acute endocarditis we find the delicate, sensitive lining of the cavities roughened. Coagulation of fibrin may follow upon the inflamed surface and becomes deposited in shreddy-like granular elevations. Fibrinous clots may form and early death result.

The subacute endocarditis is the most common form of the disease and it may not become appreciable for several days. It is usually confined to one or more anatomical divisions of the 
. 

heart and all the successive morbid changes follow each other in comparatively slow processes.

When vegetations form upon the inflaned membrane, portions are liable to break off and be carried elsewhere by the blood, thus constituting emboli, which are capable of suddenly plugging certain vessels and thereby interrupting important functions. In most cases of either acute or subacute cases of endocarcitis the most alarming symptoms disappear in a week or ten days but they usually leave the heart in such an impaired condition that the circulation is abnormal for a long time afterward. These changes usually consist of thickening or induration of the membrane lining the cavities of the heart. Especially do these changes persist if the valves of the heart have been involved in the inflammation. Even a slight attack of inflammation will render them less flexible than normal so that the free passage of the blood is interfered with and furthermore they cannot close up tightly so that some blood escapes at each contraction. The resultant efforts of the heart to compensate this condition leads to hypertrophy of its walls, more particularly of the left wall, thereby producing fullness of the capillaries in the lungs, pressure upon the air cells, difficult or asthmatic breathing and in a few months complete disability. The weak heart and inability to work finally rest1lts in a dropsical condition of the extremities and passive congestion of the kidneys may follow. Valvular disease follows a large per cent of cases of acute endocarditis, which is of itself seldom fatal in its early stages.

Semeiology.-There may be a chill with a sudden rise of temperature. The heart beats hard and the pulse is irregular. If the animal is compelled to trot there is evidence of great pain, difficulty in breathing and shortness of breath. If the valves of the right side are affected we shall find a jugular pulse. The pulse is always fast. The animal is not disposed to eat much, the surface of his body is cold, mucous membranes may be cyanotic and in nearly all cases there is suppression of the urinary secretion. In fatal cases death occurs about the fourth day.

Endocarditis may be suspected in all cases where the symp- 
toms of cardiac affection are associated with rheumatism, influenza or any septic condition.

Endocarditis may be distinguished from pleuritis by the absence of any friction murmur, absence of pain when the chest wall is percussed and the absence of effusion in the cavity of the chest. It may be differentiated from pericarditis by the absence of friction sounds and want of an enlarged area of dullness on percussion.

Treatment.-Blood-letting is contra-indicated. If you can purify the blood the heart may recover. Give antiseptics, such as salol, hyposúlphite of soda, bicarbonate of soda, etc. To guard against the chronic induration of the valves give iodide of potash. This should be given in 1 to 2 dram doses early in the disease and may be repeated two or three times a day for several weeks. In all cases see to the hygiene-good nourishing food and warm quarters. Absolute rest is necessary.

Endocarditis is usually fatal. In the autopsy there are black patches on the lining of the heart. These may extend around the valves.

Acute valvular disease cannot be distinguished from endocarditis, and chronic valvular affections are generally the result of endocardial inflammation. The valves of the left side are the ones most often affected,- the mitral and the aortic semilunar. The lesion may be mere inflammation and swelling or it may consist of organized vegetations upon the surface of the valve. Adhesions may occur and the chordæ tendinæ may be shortened or lengthened. The fibrous tissue may become changed into cartilage or bone or it may become the seat of lime deposits, resulting in ulceration, ruptures and fissures. If the heart dilates too much there may be atrophy and shrinking of the valves.

Symptoms.-Valvular disease may be indicated by a venous pulse, by a jerking, irregular and intermittent pulse, by palpitation and dyspnœa, attacks of vertigo, congestion of the brain and dropsical swelling of the limbs. Simple dilatation of the heart usually accompanies the valvular disease.

Treatment.-When the pulse is irregular or irritable, iron, gentian and ginger may be given. For the jerky violent action of the heart give 20 to 30 drop doses of digitalis or of veratrum 


viride. The potassium iodide and general tonics are of temporary benefit and stimulants should be given when the animal is weak. Very few animals recover and remain useful for any length of time when the valves of the heart have once been involved in organic disease.

\section{MYOCARDITIS.}

Myocarditis is the inflammation of the heart muscle. This disease sometimes accompanies pyæmia and septicæmia. It can be traced to foreign bodies, especially in cattle. Foreign bodies may be swallowed and passed through the walls of the stomach into the muscle of the heart and there set up an inflammation. Strangles may cause this inflammation in the horse. It usually terminates fatally and at autopsy we find abscesses in different parts of the body.

The myositis is shown by the inability of the heart muscle to contract forcibly and it results in a weak, rapid, soft pulse with irregular heart sounds. The course of the disease is very rapid and terminates suddenly from paralysis or rupture of the heart.

The heart muscle at post has a yellowish; boiled appearance and is so friable that it tears easily. Small abscesses may be scattered through it. If the disease is of long duration and becomes chronic, the fibrous tissue may increase to great extent, extending through the muscle in every direction and largely replacing it. This makes the wall of the heart very hard.

Treatment.-Quiet is of the greatest importance. The animal should be kept in an airy stall, his legs should be well rubbed and bandaged with flannel. Aid the heart by the use of tonics and stimulants. Strychnine in one grain doses twice daily, whisky in 4-ounce doses every two to four hours and digitalis in 1-dram doses every three to six hours are indicated remedies.

\section{HYPERTROPHY AND ATROPHY.}

Hypertrophy of the heart means enlargement and it occurs with or without dilatation of the cavities. This unnatural condition may be general or local, over one ventricle or both or in- 
volving only part of one. It alters the shape of the heart, which has a more nearly round appearance when hypertrophied. The left ventricle is the most likely to be involved, probably on account of the larger amount of work it has to do. The enlargement may be due to the dilatation of the cavities or it may be due to increased thickness of the walls. In draft horses both are likely to occur together. Ossification of the valves may occur.

Simple dilatation is dilatation of the cavities, principally the right ventricle, without thickening of the walls. Sometimes even a thinning of the wall of the right ventricle is seen.

Simple hypertrophy is the condition in which the ventricular walls are thickened but the cavities are normal in size.

Eccentric hypertrophy implies both the thickened walls and the dilated cavities.

Concentric hypertrophy implies the thickened walls and the contraction of the cavities.

Hypertrophy with dilatation is by far the most frequent form of cardiac enlargement. The heart may be three or four times its natural size.

Etiology.-Long-continued, severe exertion always causes cardiac enlargement. - It is seen in fast horses, especially trotters and pacers that have done hard campaigning for three or four years, and aged hard worked draft horses. This hypertrophy gives little or no inconvenience unless the animal is attacked by some other disease, then he is more liable to succumb than if he had a normal heart. Inflammatory diseases with high fever, especially pneumonia, is liable to produce simple dilatation. The concentric hypertrophy follows long continued over-exertion. The horse usually dies from some disease aggravated by the weak condition of the heart.

Semeiology.-The heart in simple, eccentric and concentric hypertrophy gives a powerful impulse at each beat; pulse full and strong. In simple dilatation it gets weaker and weaker until severe dyspnœa results. If the pulse is small and feeble at the jaw we may conclude that there is some cardiac weakness, probably simple dilatation.

Treatment.-In simple dilatation and other cardiac weak- 
, 

nesses give the animal rest and keep from excitement. Digitalis, strychnine and other stimulants are indicated.

Atrophy is the contracting and wasting of the muscular substance of the heart. The heart grows smaller arid harder and the muscle fibers lose their striations. In the muscle protoplasm are to be found around the nuclei brown pigment granules and in severe cases the pigments may lie loose between the fibers. This condition is called brown atrophy of the heart.

The chief symptom is weakness of the pulse. In the horse the pulse could not be taken at the jaw and as the consequence changes are apt to occur in any part of the body.

\section{FATTY DEGENERATION.}

This form of degeneration may involve the whole organ or it may be limited to patches, which would give the heart a mottled appearance. When the condition is general the heart is flabby and in extreme cases collapses when cut. Fatty infiltration deposits fat between the fibres while in degeneration the muscle protoplasm itself is changed into fat. Fatty degeneration is often associated with other morbid conditions such as obesity, dilatation, rupture, aneurism, etc. When it exists alone its presence is seldom suspected previous to death. It may be due to deteriorated conditions of the blood in wasting diseases, excessive hemorrhages, or to poisoning with arsenic and phosphorus. Fatty infiltration co-exists with obesity.

Symptoms.-The most prominent symptoms of fatty degeneration are a feeble action of the heart, a remarkably slow pulse, general debility and attacks of vertigo. It may involve a liability to sudden death from rupture of the walls.

Obesity of the heart is the deposit of immense quantities of fat around the heart. The result of this is a very weak heart, shortness of breath, which runs into dyspnœa. The animal may be all right when standing still but as soon as exercised the trouble shows. 


\section{POLYPI OR TUMORS OF THE HEART.}

Tumors may develop on the inside or outside of the heart. On the inside they are more likely to take the form of polypi. They are usually orginated from vegetations attached to the base of the valves. These polypi may become regular fibrous growths such as are seen following an inflammation. They are more often found upon the lining of the auricle than upon the ventricle. Fragments of these vegetations often float off as emboli and lodge in some other part of the body, as the brain, where they produce degeneration, or coma and finally death.

When these tumors form in the heart they can be diagnosed by auscultation; a rasping sound reveals their presence.

Vascular tumors are frequently found on the outside of the heart at the apex. Sometimes they contain hydatids.

\section{RUPTURE.}

Rupture of the heart may occur as the result of some previous disease, such as fatty degeneration, dilatation with weakness of the walls, etc. It may be caused by external violence, a crushing fall, pressure from some great weight, etc. Jumping sometimes causes it.

The rupture more frequently occurs in the left ventricle, although it may occur in the auricles. Death quickly follows a rupture.

Sometimes the heart is found congenitally misplaced. It is sometimes on the right side and sometimes in the front outside of the chest cavity, or in the abdomen. This condition of the heart is called Ectopia Cordis.

\section{DISEASES OF THE BLOOD VESSELS.}

\section{ARTERITIS.}

Inflammation of the lining of an artery is called arteritis. This inflammation sometimes spreads to the media and adventitia and it is hard to find what causes it. The strongylus armatus is 

often found right under the coat of the vessel where it sets up an inflammation. Other worms may cause this condition. The iliac artery in females is sometimes injured in parturition, setting up ill results.

The most common result is the formation of a thrombus at the point of inflammation. It may grow so as to obstruct the vessel and it is then spoken of as thrombosis of the artery. The partially organized lymph may become detached and float off to some other part, as an embolus. When an embolus reaches a vessel too small for it to pass, it becomes lodged and plugs the vessel, producing embolism.

Treatment.-Embolism is incurable and thrombosis may produce serious results. Thrombosis must be treated with hot or cold applications externally and internally-acetanilid, quinine and belladonna. In case of arteritis of the iliacs give a laxative and cold rectal injections ( 60 degrees F.) with a little belladonna in it. Keep the animal warm in cold weather.

\section{ANEURISM.}

Aneurism is a dilatation of an artery and it is sometimes called a pulsating tumor. This may be the result of one or two causes. First may be mentioned weakness of the arterial walls, which the pressure dilates. The walls may get very thin and sometimes rupture and the animal bleeds to death. This condition usually occurs in the posterior aorta. Worms may cause aneurism, especially in the colic artery.

If the aneurism is external, apply pressure in the form of a truss. If it is in a vessel that can be dispensed with, ligate it. It may occur from stricture caused by arteritis, the dilatation being anterior to it.

\section{PHLEBITIS.}

Inflammation of the veins, is called phlebitis and it is either traumatic or idiopathic-idiopathic as regards its origin. In the horse it is usually traumatic. As the result of the inflammation there is extensive exudation into the vein, which finally becomes 
obliterated at the point of injury. More or less pus is discharged.

The idiopathic form is seen in the human, but seldom or never in the horse. In the human it is associated with milk leg at times.

Symptoms.-Local heat and swelling. If the vein is obliterated, it is like a cord.

Treatment.-Ordinary antiseptic treatment. If there is a suppurating wound present, it must be curetted and treated freely with antiseptics.

\section{VARIX.}

Varix is a dilated vein, sometimes called varicose veins. It is due to obstruction or weakness of the venous walls. Varicosity of the jugular is sometimes met with, especially where the two jugulars meet. A varix of the jugular is sometimes seen in the cite of a goitre. It may be mistaken for a goitre, but it is softer and is quite compressible. A varix of the saphena major is often seen in connection with a bog or bone spavin.

Treatment.-Pressure is about the only treatment. In the human, elastic stockings and bands are used on the legs. In the case of bone spavin you will find the saphena major enlarged. In firing, be careful not to press the iron too heavily when over the part. In case of bog spavin it is sometimes ligated.

\section{DISEASES OF THE URINARY SYSTEM.}

The chief function of the kidneys is the removal of the surplus nitrogen of the body in the form of urea, and the surplus water. Most of the poisons of the body also those of disease are thrown off through the kidneys. The kidneys, therefore, are the sanitary scavengers and purifiers of the system, and when their functions are impaired or arrested, the retained poisons quickly show their presence in the resulting disorder of the skin, nervous system, and in fact all the other organs. On the other hand, scarcely an important organ of the body can suffer derangement without a corresponding disorder of the urinary system. 

The prominent causes of urinary diseases may be summed up as follows:

All extensive inflammations and acute diseases attended by fever diminish the liquids of the urine and increase the solidswaste products-resulting in the irritation of the urinary organs or the poisoning of the system at large by the retention of the surplus waste.

Diseases of the heart and lungs, by interfering with the free onward flow of the blood from the right side of the heart, tend to throw that liquid back upon the veins, and this backward pressure of venous blood reacts upon the kidneys.

Poisons in the food and water (such as irritant diuretic plants, musty hay or oats); green vegetables covered with hoar frost, excess of phosphates in such food as bran, peas, lentils, vetches, rape cake, cotton-seed cake, etc., deprivation of water, producing too great concentration of the body tissues,- - these are direct causes of disturbance in the kidneys.

A disordered liver producing an excess of bile will color the urine; an excess of hippuric acid and its allied products favor the formation of calcareous deposits; of taurocholic acid, the destruction of the blood elements and consequent irritation of the kidneys; of glycogen, the production of saccharine urine.

Any disorder leading to impaired function of the stomach is causative of an excess of hippuric acid, of bile, of oxalic acid, of sugar, etc., in the urine, resulting in irritation of the urinary passages.

Diseases of the brain and nervous system, notably of the base of the brain and the spinal cord, induce various urinary disorders, such as chylous urine, diabetes, and albuminuria.

In some cases the changes in the urine are the sole sign of disease. In health the horse's urine is of a deep amber color and has a strong odor. On a feed of grass it may show uniform transparency, while on a grain and hay ration there is an abundant white deposit of carbonate of lime. Of the morbid changes the following are to be looked for: (1) Color; white from deposited salts of lime; brown or red from blood clots or coloring matter; yellow or orange from bile or from blood pigment; pale from excess of water; or variously from vegetable ingredients 
(santonin makes it red; rhubarb or senna, brown; methylene blue, tar or carbolic acid, green). (2) Density: The horse's urine may be 1.030 to 1.050 , but the specific gravity may exceed this, as in diabetes, or it may sink to 1.007 , as in diuresis. (3) Chemical reaction: The horse on a vegetable diet has alkaline urine, while in the sucking colt or in a horse fed on flesh or on his own tissues, as in starvation or abstinence during disease, the urine is acid. (4) Organic constituents: Albumen, mucous shreds, casts, worms, etc. (5) Salts: These crystalize out spontaneously as the result of excess of some acid or base in the urine. Pus and an excess of mucous are frequently found in the urine .

\section{ALBUMINURIA.}

In the horse this can be safely called equine Bright's. It occurs in two forms, acute and chronic. It is mostly due to indigestion. The chief symptom is the presence of albumin in the urine, but sometimes none may be present. Accordingly, several tests should be made for the determination of the albumin. You cannot rely on a single sample. Tube casts are also present. These are cylinders of fibrous coagulum, the coagulation having taken place in the uriniferous tubules to which the casts conform in shape. In the case of fatty degeneration the casts will have a waxy appearance, due to the presence of fat and oil in them.

Tests for Albumin in the Urine.-Put some of the urine in a test-tube and boil it: if any albumin is present, a white coagulum will form. Heating the urine will also precipitate phosphates if in excess, but nitric acid will dissolve them. If the urine is strongly alkaline, neutralize it with a little acetic acid, for alkaline urine may coagulate when heated even though albumin is not present. Another test consists in placing a little urine in a test-tube and carefully pouring down the side of the tube a little strong nitric acid-pour it in such a way that it goes to the bottom of the tube underneath the urine and a white line of coagulation will form between the acid and the urine.

Semeiology.-The first indication of equine Bright's is stiffness in the gait of the horse-of the hind parts. In old confirmed cases they step about eight or ten inches to a step, are stiff 

in turning and inclined to stretch. Ultimately the horse will suffer constitutionally, - animal will become hide-bound, coat long, becomes. weaker and weaker in the hind parts, finally gets down and is unable to get up, delirium follows, then uric acid poisoning and death.

Treatment.-The treatment is usually not applied early enough for the disease is not then diagnosed. But if it can be detected in its early stages, the stomach should be given a complete change of food. If possible the animal should be put on grass. Give him stomachics and saline laxatives. Exercise regularly. Give gentian, nux vomica, arsenic, zingiber, with a little charcoal. Diuretics in any form are not indicated. Give plenty of bran and sloppy food. After a few weeks' time change to a sour tonic.

$\mathbf{R}$

Fl. Ext. Gent. Rd. Pulv................ounce $j$

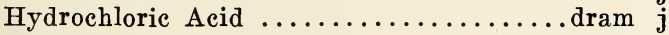

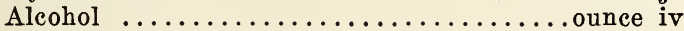

Aqua qs. ad......................... $j$

M. Sig. - 1 ounce 3 times a day before eating.

After using this treatment for a week or ten days go back to the other treatment.

\section{HEMATURIA.}

Hematuria is bloody urine but not a high-colored urine necessarily. The blood is usually passed in clots and they can be seen on the floor.

Etiology.-Severe strain of the loins, cystic calculus, strong diuretics, various parasites in the kidneys, especially the strongylus gigas, congestion and degeneration of the kidney and cancers, especially melanosis, and blows across the loins, are the principal causes of this disease. The strongylus gigas seems to be partial to the kidney and develops enormously there. A male 10 inches long and a female 14 inches long were taken from the kidney of a dog that died in the Chicago Veterinary College.

Treatment.-Treat as the symptoms indicate. If the disease is caused by strain, apply cold applications and keep up for two or three hours. If this fails to do any good, give the horse a 
cold water injection and run the hose in three or four feet. If a calculus is found in the bladder it must be removed. If the hemorrhage continues and no calculus in the bladder has been found nor any bruise, the cause may be a renal calculus. Hydrochloric acid internally will reduce the alkalinity of the urine. In case of parasites nothing can be done.

\section{DIABETES INSIPIDUS.}

Diabetes insipidus or polyuria is profuse micturition and associated with it is great thirst. The urine is clear as water and horse passes it every fifteen to thirty minutes. Stable men call the condition flooding. The specific gravity of the urine is about the same as water-1.002 or 1.003 . In horses clear urine is frequently a sign of blood poisoning, as in glanders.

Etiology.-The chief cause is indigestion, or mal-assimilation of the food. Improper food, such as musty oats or hay or kilndried oats, or too free use of diuretics may be the cause of the trouble.

The horse will run down in flesh, he develops a long staring coat, gets wabbly behind and finally dies from collapse. The appetite usually remains good.

Horses generally recover but mules do not. Post mortem shows a general pallor of all the parts; the kidney, especially the cortical portion, is soft.

Treatment.-Iodine is the best remedy and should be given in large doses. To a 1,200 pound horse give a dram of the iodine crystals in a linseed bolus. This quenches the thirst and incidentally reduces the flow of urine. In the horse one dose is usually sufficient; if not, repeat in three or four days. Following the dose of iodine, treat the indigestion. Give bicarbonate of sodium,-a dram three times a day for a week and then change to hydrochloric acid for a week. Then go back to soda. Give vegetable bitters and feed on grass or bran. Do not let the horse bolt his food.

'A mild form of polyuria is seen in horses fed continuously on hay and oats without any change. The common remedy is a tablespoonful of starch in the food three times a day, for a day 

or two. Give bran with the oats and a mild purgative if the animal is not working.

\section{DIABETES MELLITUS.}

Diabetes mellitus (glycosuria) is a condition where sugar is found in the urine. It is comparatively common in the human but it is seldom found among the other animals. Dogs fed exclusively on liver develop fatty degeneration of the liver and it is suspected that they die from glycosuria. Professor Williams says that he never saw this disease in the horse or in herbivorous animals.

Etiology.-The predisposing cause is continuous over-eating. The disease is a result of indigestion.

Semeiology.-The urine is excessive in amount and of high specific gravity. Next symptom is lassitude. Then follows swelling of the feet and red spots on the skin of the legs. These spots become larger and finally break out in raw sores.

Treatment.-All starch and sugar should be withheld from the food and the diet should consist of meat and nitrogenous foods. Give plenty of water. Avoid irritating substances.

Besides sugar in the urine, pus may appear, also mucus in increased amount. The mucus may be seen as a floculent cloud which is easily shaken up when the urine is in a bottle. It never settles. Pus settles to the bottom of the vessel in a whitish yellow deposit, which is easily shaken up. Unless the pus is very abundant it need give no alarm. It may be caused by calculi or some irritating substance in the food or medicine. The urine must be examined microscopically to determine its presence. In order to clear up the urine, remove the cause of the irritation. Give the animal something to allay it and also give him a laxative. Give the horse fluid extract Saw Palmetto in ounce doses three times a day.

\section{OXALURIA.}

Oxaluria is a disease seen in horses oftener than in any other animal. It is a condition in which the oxalic acid if formed in 
the body crystallizes out in the urine in the form of oxalate of ime. Oxalic acid itself is a product of imperfect combustion of the nitrogenous and amyloidal substances of the body. This condition is found to be comparatively common in animals which are overfed and are given irregular work. Fatiguing labor after a period of idleness and high feeding will cause it. The most aggravated cases are found in trotting stallions.

Symptoms.-Great dullness- and languor are marked symptoms. In severe cases there is rapid loss of flesh,-a difference in flesh can be seen in a horse within a week. He has a capricious appetite. On examination nothing can be found. The anima! runs from bad to worse and gets stiff in the loins. In from ten to 'fifteen days a bran-like scurf appears on the skin. This is a prominent symptom. In four or five weeks, if the disease runs that long, he becomes hide bound and emaciated. He passes water oftener than normal and the urine is of pale amber color. The urethra becomes scalded by the urine and he dreads to micturate. The mouth is furred, has a sour smell and a soapy feel. The bowels are irregular. Upon testing the urine the reaction will be neutral, but after standing a little while it will be alkaline. Examined microscopically oxalates of lime will be found, -octahedral crystals in the horse and dumb-bell shaped in the dog and human. These crystals are soluble in nitric acid without effervescence, but not in water and are not affected by boiling in acetic acid or by potassium hydroxide.

Treatment.-Indigestion being the cause of the condition, give the animal a complete change of diet and withhold all saccharines. Give him bran, grass, etc., and gentle and regular exercise. Drinking water should be pure, rain water is the best. In addition to this give mineral acids, hydrochloric acid. Give Saw Palmetto. If he is in much pain, give a little morphine or codine and combine a little belladonna with the palmetto.

\section{RENAL CONGESTION.}

Renal congestion is hyperemia of the kidneys. It may occur as a sequel of any debilitating disease. It may be the result of irritating substances applied to the skin or being present in the 

food. Absorption of cantharides, which has been spread over a large surface, or too much turpentine admiristered internally, exposure to cold and dampness, accidental injuries or violence, blood contamination, - these are the principal causes of renal congestion and in all cases the Malpighian tufts of the kidney are congested. In Azoturia passive congestion occurs.

Post Mortem.-The kidneys are very large and red; there will be ecchymoses corresponding to the Malpighian tufts sometimes on the surface.

Treatment.-Ascertain the cause if possible and remove it. If too much turpentine has been given, give the horse a small dose of oil and saw palmetto. Belladonna is indicated internally. Apply hot rugs to the loins and give perfect rest for a few days and a light diet. Nephritis usually follows.

\section{NEPHRITIS.}

Nephritis is inflammation of the kidneys. There are two forms. The first form is the mild or subacute. It follows some debilitating disease such as influenza,-in fact any weakening disease in which the fever has been high for some time, say a week. Congestion occurs around the loops of Henle and the Malphighian tufts and is due to the accumulation of debris in the system resulting from the previous fever, during which the kidneys were inactive.

Pathogenesis.-High fever lasting for about a week without diuresis being produced. The kidneys fail to remove the accumulating debris and become congested to the extent of inflammation as a result. This is Bright's disease of the kidneys. It is seen most often in the dog, cat and human, but seldom in the horse.

Post Mortem.-The kidneys are found congested.

Symptoms.-The animal seems to be convalescent from the fever which first attacked him when suddenly the appetite fails, the urine becomes scanty and high-colored, in some cases quite red. Upon testing it albumin is present. Emaciation takes place and general debility comes on rapidly. There is a staring coat, œdema of the legs and stiffness of gait. 
Treatment.-Hygiene should be good; give scalded oats and bran, equal parts. The kidneys must be made to work or the horse will die. Diuretics are strongly indicated; at the same time keep the bowels slightly relaxed with sulphate of sodium. When the disease becomes chronic the animal will die but he may live for two or three years.

The second form of nephritis is the acute parenchymatous. This form come on suddenly with a tendency to kill in about five days or produces one or more abscesses, later. If it attacks both kidneys it will kill in five days, but if only one is affected the horse may live for some time and possibly recover.

Etiology.-Anything that causes renal congestion may cause acute parenchymatous nephritis. Sometimes severe strains of the psoas muscles cause inflammation which extends to the kidneys. The most typical cases come from cold and dampness.

Symptoms.-The horse is in great pain and has an anxious countenance, glistening eyes, and although restless is disinclined to move and yet may sweat. His temperature may run up four to six degrees. He does not seem to wish to move his hind legs and stands persistently. He passes only a small quantity of urine and it is hot and high-colored. If pressure is put against the affected part there is pain. If the horse is forced to move he has a straddling gait. There is great loss of appetite. He grows rapidly worse and may die in the course of three days, but the general average is five. The urine is entirely suppressed before death. The form of death is syncope.

Post Mortem.-In a very acute case without pus the kidneys will be swollen and dark red. Occasionally you will find part of one or both gangrenous.

Treatment.-This form of acute nephritis requires prompt treatment. Put hot wet rugs over the loins and change them every half hour. Keep the animal where he is warm and comfortable. Give rectal injections of warm water with a little belladonna in it,-Fl. Ext. Bella, half ounce to water four pints. Give horse linseed tea to drink, acetanilid in moderate doses as often as he can stand it and aconite in small repeated doses. No matter what the treatment, however, the animal very seldom, if ever, recovers. 



\section{RENAL CALCULUS.}

A renal calculus is a calcareous secretion which is usually found in the pelvis of the kidney. It may be in the form of stone of considerable size or it may be in the form of gravel, which floats down through the ureters with the urine into the bladder. The calcareous material may lodge in the ureters or may remain in the bladder to form large stones. In case the ureter becomes obstructed the pelvis of the kidneys will become distended, local inflammation will follow with more or less suppuration. Suppurative nephritis will be the ultimate result unless the stone is dislodged. A horse in this condition would die from a combination of pyæmia and uræmia and coma would be the form of death. The stone is composed of carbonate and phosphate of lime, the carbonates predominating.

Symptoms.-Colicky pains at irregular intervals are present, especially after severe exercise. The urine is exceedingly heavy, being charged with earthy salts. Occasionally there is some blood in the urine and it will clot on the floor. Upon examination of the ureter through the rectum you can detect the distension and will feel the soft, fluctuating enlargement which can be traced to the kidney.

Treatment.-Give the horse pure water to drink (rain water is the best). Give plenty of bran and grass in season and hydrochloric acid in dram doses once a day in a pint of water.

\section{CYSTITIS.}

Cystitis is inflammation of the lining of the bladder.

Etiology.-The inflammation may result from calculi or from gravel. The most common cause is irritation of the mucosa or irritating substances in the food or water. In the human it is often caused by gonorrhœa.

Semeiology.-Nervous excitement is marked at first and then depression comes on. Elevation of temperature may or may not be present. Micturition is very painful with spasms of the bladder (tenesmus). The horse urinates frequently and the urine is in small quantities, hot and high-colored, sometimes 
streaked with blood, especially if the condition is caused by calculi, or gravel. The bowels are apt to be congested and the stools coated with slime. If the cause of irritation is in the alimentary tract, then its mucous membrane will be congested and catarrhal. The disease may run a rapid course and terminate fatally in three or four days But in a mild case it may run three or four weeks.

Treatment.-General anodyne course should be followed. If the inflammation is caused by a calculus, remove it if possible. The anodyne course is to give him linseed tea with rain water. To keep up the strength of the horse, give him raw eggs and milk and if he will eat it, grass. If the cause of the irritation can be removed, then put the animal on stimulants such as strychnine, nitro-glycerine, etc. Flush the bladder with a saturated solution of boracic acid two or three times a day. The catheter should be sterilized and the solution should be run in from a fountain syringe. Cramps may occur, but when they cease, go on with the treatment. Keep the bowels open with a laxative and give rather liberally saw palmetto or san metto.

\section{DYSURIA.}

Dysuria is the painful passage of urine. This may be caused by the partial obstruction or irritation of the mucous lining of the urethra. The most aggravated cases of this is seen from gravel in the form of cystic calculi or smaller grains floating down the urethra. In males the next most common cause is enlargement of the prostrate glands. It generally comes in old age, and is attributed to too much stud work. Occasionally, but rarely, it is seen in geldings. Dysuria is sometimes caused by a sabulous concretion in the fossa navicularis, called by horsemen the "bean." It is sometimes so great as to press upon the urethra. Other causes of dysuria are thickening of the neck of the bladder from cystitis of the bulbous portion of the urethra, cancer of the penis, tumor of the glands penis, foulness of the sheath from the accumulation of sebaceous material, producing swelling and pressure. Another cause is the horse urinating in his sheath either from habit or malformation. In the female, 

at the opening in the vagina there is often found a small tumor, called a caruncle, which extends sometimes into the urethra and makes micturition slow and painful. Prolapsus of the uterus may be a cause; hardened feces may press down upon the vagina and urethra. In the male stings of bees may cause swelling of the sheath.

Treatment.-If possible locate the cause. This is sometimes very difficult. In case of enlargement of the prostrate in the dog or stallion the old treatment consisted in applying tincture of iodine to the perineum and giving idodide of potash internally for a long time. But this is more or less injurious to the reproductive power of the animal. Pass the catheter occasionally: in bad cases it may be passed two or three times a day. In the human the prostrate gland may have to be removed in part, but this is a very dangerous operation. In case of thickening of the neck of the bladder give external treatment that will allay the irritation of the mucous membrane of the bladder, which is often due to the acid condition of the urine caused by eating too much meat. In such a case alkalies will overcome the acidity and relieve it. Retire the animal from stud work according to the severity of the case. In foulness of the sheath, wash the parts with soap and water and introduce a wad of absorbent cotton dipped in carbolized oil. Swab out once a day and at each dressing bring the penis down, pulling gently and slowly.

\section{ISCHURIA.}

Ischuria is suppression of the urine. There may be none secreted or there may be inability to pass it if it is present. When the latter is the case it is usually from paralysis of the muscular coat of the bladder.

This condition usually exists when the horse is kept too long without giving him a chance to urinate. Some horses are peculiar as to when and how they do this, so never fail to humor them in their peculiarities in this respect. Therefore, when you notice colicky pains, switching of the tail, and restlessness you can suspect the cause. Many horses dislike to urinate while down; therefore in azoturia the water should be drawn from 
them. When a horse is to be raised with slings, never fail to draw the water from him before raising him or rupture of the bladder may occur. When no urine is secreted as in purpura hemorrhagica, then the kidneys are at fault.

Treatment.--When the urine is not secreted diuretics and diffusible stimulants are indicated. If there is spasmodic contraction of the neck of the bladder, then anodynes should be given,- -as hot water and belladonna. Try passing the catheter smeared with solid extract of belladonna. This will often relieve the constriction. If you are sure of paralysis being present, give one dram of powdered nux vomica in the feed night and morning for'a few days.

The condition should be treated promptly and the animal not neglected. Otherwise the case may become serious.

\section{ENURESIS.}

Enuresis is incontinuence of the urine. When the animal cannot retain the urine in the bladder it dribbles away as it forms. The condition is usually due to paralysis of the neck of the bladder; the muscles fail to contract and the urine dribbles down the legs, scalding them. It is sometimes very painful. In the male the penis becomes paralyzed and hangs down.

Treatment.-Nerve tonics are indicated, with local shocking such as dashing cold water on the perineum. This often resuscitates the waning power. A gallon of water given per rectum produces a shock and this comes in good in this condition. Nux vomica taken continuously for a month will promote the general health.

Strangury is the passage of the urine in drops on account of severe irritability of the mucous lining of the bladder or of the muscularis. The urine comes away every few minutes in drops or in small quantities. Apparently there is hyperasthesia of the bladder mucosa. The retention of the urine causes inflammation of the bladder.

The treatment must be toward allaying the irritability. Therefore give anodynes such as opium, belladonna, etc.; and laxatives,-oil and linseed tea to drink, 
$$
\text { . }
$$ 



\section{URETHRITIS.}

Inflammation of the lining of the urethra is called urethritis. Any of the causes producing inflammation in the bladder may cause urethritis. If a stallion copulates with a mare suffering from leucorrhœa, urethritis is apt to follow.

Treatment.-Mild soothing diuretics and stimulants in the form of copaiba, 1 dram dose three times a day, or oil of sandalwood are good remedies. Suspend the horse's stud work. Be careful about using local injection. A solution of bichloride of mercury 1 to 2000 can be used and twenty-four hours after another solution 1 to 6000 . Do this twice a day for two or three days. In all these cases relax the bowels with the salines. As a rule the inflammation does not extend further up than three to six inches, but if it does, it is due to the irritation of gravel in the bladder. If the condition arises in a stallion, retire for a week or two. Clean the parts with soap and water three times a day and at the same time syringe mild antiseptics into the urethra. Solution of sulphate of zinc two grains to an ounce of water is the best. Do not manipulate the penis after injection.

Rupture of the bladder may occur occasionally but it is very. rare. It is more likely to occur in the human than in the other animals. It may be seen in cases of azoturia or in cases of long rentention of the urine, or it may be ruptured by raising a recumbent horse with slings without first emptying the bladder. It is always fatal.

Eversion of the bladder often occurs. The bladder protrudes through the vulva, appearing like a case of prolapsus or a polypus. Examination will tell the difference. The lining of the bladder is velvety and soft and the openings of the ureters can be found. Return the bladder to its proper position with a probang and give anodynes and oil.

\section{TUMORS.}

A tumor may be defined as any prominence or growth, unnatural, on the body or in any organ or gland. It may be composed of the same tissue as where it grows, or it may be en- 
tirely dissimilar. A tumor grows by cell-proliferation, the same as other parts of the body; new blood vessels and lymphatics extend into the growth and furnish it nutriment. These nutrient vessels are usually much enlarged. As a rule nerve fibers extend into a tumor. Tumors are subject to all the changes which occur in any other tissue, such as fatty degeneration, suppuration, ulceration, gangrene, pigmentation, calcification, etc. Necrosis sometimes takes place, often to such an extent as to entirely destroy the tumor.

Tumors are of various shapes and forms: nodular, when resembling nodules; tuberous, when like a tuber; fungoid, when cauliflower shaped; polypoid, when like a polypus pear shaped; papillary, when they are shaped like a papula; dentritic, when they have roots or branches. Tumors may be single or multiple; this is particularly seen in black cancer, such as is seen around the tails of white horses-the melanotic tumors.

Tumors are either malignant or benign. A malignant tumor has the following characteristics: (1) It invades the surrounding tissues by peripheral or eccentric growth; (2) it has a tendency to recur locally after removal; (3) it will spread to other parts of the body, by metastasis; (4) it has a tendency to interfere with the nutrition and well being of the body and results in cachexia.

The real cause of tumors is not known. Predisposing causes are such as heredity, mechanical injuries, parasitic invasion,-in fact anything that will lower the resistance of the animal. Conheim developed the theory that tumors are due to embryonal displacement,-either to misplaced cells or to superfluous cells. Tumors do not develop in young animals so much as in old. The melanotic tumors in horses do not develop usually until the age of ten or fifteen years.

Tumors may be divided into six large classes:

1. Connective tissue tumors (histioid).

a. Fibroma.

b. Myxoma.

c. Sarcoma-round, spindle-celled, mixed, and giant-celled.

d. Endothelioma. 


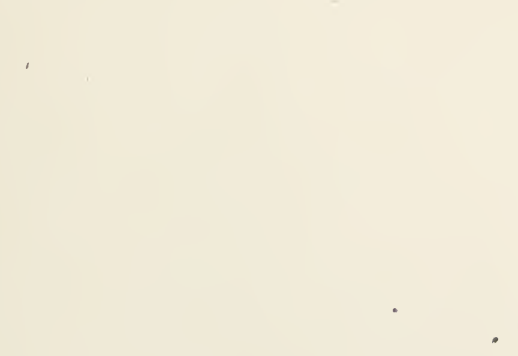



e. Lipoma.

f. Chondroma.

g. Osteoma.

h. Glioma.

2. Myomata.

3. Neuromata.

4. Angiomata.

5. Epitheliomata.

6. Teratomata. These are mixed tumors, which are composed of all kinds of tissue. They are congenital. Teeth and hair may be found in them.

Tumors of the first and fifth classes are most likely to become malignant. A malignant tumor is called a cancer, and there are several different kinds, such as hard, soft, pigmented, etc. A hard cancer is called a scirrhus cancer, especially by the old writers. A scirrhus cancer is very hard and dense and consists of white tissue. It is sometimes of uneven hardness; other times it has hard projections extending from it into the tissue to which it is attached. The tumor cuts like cartilage and from the cut surface a persistent hemorrhage is prone to occur. When operating it is difficult to arrest the hemorrhage. The scirrhus has a special liking for the lymphatic glands. Unless such a growth interferes with mastication or some other functional activity it will produce little or no harm until it begins to break down, when ulceration may take place and eventually the tissue refuses to heal. Early removal with the knife is the only treatment.

As examples of the soft cancers may be mentioned the encephaloma, fungus hæmatoid, seen growing in the eyes of cattle, medullary fungus, etc. The soft cancerous fungi may be found in any of the tissues, but mostly in the glands. In the horse it is often found on the penis and may extend to the testicles; also on the vulva in the mare. It may affect bones and may arise in the periosteum. To the touch the tumor has a soft fluctuating feel, but there is no pus in it. Upon cutting into it a profuse hemorrhage will take place. Around such tumors is a network of veins which have increased in size greatly and this 
feature makes it difficult to operate. These tumors often break down and granulate, going on to ulceration.

Colloid degeneration frequently takes place in tumors, especially in epithelial tumors. The colloid material is amber-colored and resembles half melted glue in consistency. Other forms of degeneration are the mucoid and the contents may become very fluid, forming a cyst.

Of the connective tissue tumors which become malignant the sarcoma is the most common type. The tumor cell is a round cell or spindle shaped. The round celled sarcomata are the most malignant. These tumors are very prone to metastasis. The so-called melanotic cancer is a type of sarcoma. Each cell contains an abundance of pigment, which is often of so great amount that the form of the cell, its nucleus, etc., cannot be made out. The pigment may invade the intercellular substance as well. These black tumors have every feature of malignancy. Sometimes they develop very suddenly, producing great lameness. No tissue in the body is exempt from them, but their favorite sites are the vulva, the anus, and the bare part under the tail. When removed, they come back in some other part. They are found most frequently in old white horses.

The epithelial cancer is common. This tumor is composed of epithelial cells, which can be of any type. The tumors may grow and develop in the skin and mucous membrane or in the glands. Metastasis usually follows and even though the tumor be removed, it will most always recur.

A benign tumor usually is surrounded by a capsule. It may consist of fat, cartilage, fibrous connective tissue or bone.

The fibrous connective tissue tumor is called a fibroma. It may be soft or hard. The polypus is a form of soft fibroma which is usually found in the nose. It is attached by a pedicle and in the horse it may be so long as to interfere with the epiglottis. It produces a difficulty in breathing, a sort of snoring or snuffling, the same as heard in the pug dog. The best way to remove a polypus is with a wire ecraseur. If the neck of the polypus is small, it can be twisted off with a pair of forceps. After removing, wash out with perchloride of iron-1 dram to the pint of water-twice a day. Another kind of fibroma is the 

wart. The eyelids and the legs are the usual places for warts. Not only is the connective tissue increased to form the bulk of the wart, but the surface epithelium is increased also and they may become malignant.

An interesting polypus is sometimes found in the vagina of the mare and may grow to a large size. It could be confused with an everted bladder or with an impervious hymen in fillies.

The condroma or cartilaginous tumor develops commonly on the sternum of the horse or ox, following injury. In the horses we may find one growing in the trachea, following tracheotomy or from kicks and wounds. If they are limited in size, they can be removed with the knife, but when large, removal is impracticable. In the early stage of their growth they are largely made up of fibrous connective tissue.

The so-called osteoma or bone tumor is usually an inflammatory growth and it is not a true tumor.

The lipoma or fatty tumor consists of fat and it is liable to grow in any animal and in any part. Such a tumor is easily removed; it is non-vascular. Apply a little cocaine when operating.

The neuroma is a nerve tumor and it is most commonly seen as a sequel of neurotomy. In this operation the nerve should be drawn down and cut off so that the end will draw up into the wound. Then it will not grow out into the granulations which form during the healing of the wound. You will find that when the horse is not benefited by the operation of neurotomy, it is usually due to the formation of one of these tumors.

\section{CYSTS.}

Cysts are very common and they are important. Examples are Capped Hock, Capped Knee, Wind Galls, etc. They may grow on any part of the body, inside or outside. They may contain hair and they are lined with skin as well as being covered with it. They usually follow an injury. When produced they rapidly fill with serum, and an acute inflammation takes place in from 12 to 48 hours.

Treatment.-For cystic tumors on a horse's legs the first thing to do, especially if they are of large size, is to open them. 
In capped hock it has been proven that an incission can be made with safety and it is the only satisfactory treatment. If the tumor is not tapped, eventually a fibrous growth will form, which may prove difficult to remove.

Opcration.-First find where the point of injury is and locate the size and position of the cyst by palpation. Open it with a sharp pointed bistoury, never a scalpel, as near the bottom as possible. Manipulate the tumor so as to make it bulge at the point to be incised and insert the knife slowly at the bottom, pointing it inward, upward and outward, till the point of the knife is about an inch from the insertion and then cut through. Sometimes you will not cut deep enough and the serum will not flow, then cut again a little deeper ir a similar manner. Do not be afraid if the blood flows freely, for the hemorrhage can be stopped. Syringe out the cyst with an iodine solution. This will prevent reforming. Tincture of iodine, full strength, can be used. Never syringe after the first time. Dip a wad of oakum in some antiseptic solution and insert in the sack. Bathe the wound with hot water an hour at a time and insert a fresh piece of oakum after each bathing. Keep up this treatment for 10 to 20 days, by which time the place will usually become healed. A hard fibrous swelling will usually form in the place of the cyst and the parts should be bathed with witch hazel, added to the same amount of water. Wrap the parts in flannel and give a little walking exercise. Animal will be all right in about six weeks.

If an old case is brought to you, open and make a new wound of it, poultice and after about a month put on a mild blister. If the wound tends to heal with the formation of much fibrous tissue, hand rubbing will help it very much.

Serous cysts on the knees of cattle can be opened and treated all right if they are young in growth, but if old chronic cases, the doctor would better let them alone. Where they form in other parts of the body, from kicks and injuries, etc., they can be opened at the bottom and some antiseptic be injected. Use hot applications an hour at a time and regulate the diet. In capped elbow, where the tumor is small and bagging, it can be removed by ligating. Tie a cord around it tightly and after two 



or three days another a little tighter for a day and then cut off and sear with a hot iron. Considerable inflammation is apt to accompany this method, but it is the best way.

The ovarian cyst is common in women, cows and cats. It is a dropsical condition of the ovary and it does not always involve the whole of the organ. In the human these cysts may become malignant by the growth of the epithelium lining the cavity.

Sometimes these cysts can be treated heroically by inserting the hand in the vagina and another hand in the anus and crushing the cyst between the two. The serum escapes into the abdominal cavity and does no harm. Give soft feed and rest and the animal will recover in a day or two.

Mucous cysts develop in the mucous membrane and fill with mucus instead of serum. In the mouth they are called Ranula, and they grow into long, tuberous forms. Slit them up full length and wash out with boracic acid solution.

Cysts in the thyroid gland are called bronchocele when they are filled with water, and goitre when they fill with the solid colloid substance. Goitre is very common in dogs and they frequently develop into malignant growths of the sarcomatous type.

Treatment.-In new growths, paint the parts with iodine once a day and give iodide of potash internally. If the skin gets sore, cease the treatment for a time and then continue again. It is a dangerous operation to attempt to dissect such a tumor out. It is, however, an operation that is becoming more common every day, especially in the human. Goitre is also very common in the stallion. Thyroid extract is used in the treatment of goiters in dogs with much success.

Dentigerous or teeth-bearing cysts are often found. Tumor teeth may be found most anywhere, but frequently at the base of the ear, sometimes in the maxillary sinuses or the frontal and also in the testicles or ovaries. When they develop at the base of the ear, there will first be seen a little swelling, which will grow suddenly and finally rupture. Upon cutting it through a molar tooth may be found which seems to grow without a matrix.

Certain inflammations may result in hard fibrous swellings which become more or less permanent. The so-called scirrhous cord is one example. It is not a true tumor, but is the result of 
inflammation following castration and the botryomyces is supposed to play a role. Some part of the fibrous mass will usually give evidence of an abscess in the wall of which the fungus can be found. Several different kinds of these botryomyces have been described and probably no particular kind is specific. The fungus seems to be a very large sized coccus, which is found in masses much like the zoogloea.

Muscle tumors or myomiata, are not very common. If a proliferation of the non-striated muscle they are called leiomyoma; if of the striated, rhabdomyoma. Leiomyoma is the most common. In the horse leiomyoma of the kidney has been reported and in the sheep, of the liver. In the human a common tumor is the uterine 'fibroid, which is a mixture of non-striated muscle and fibrous connective tissue. Leiomyoma of the ovary is rare.

\section{DISEASES OF THE BONES.}

All of the lower animals are subject to diseases of the bones. The horse especially is more prone to bone disease because of high feeding and rapidity of gait. In health bone is insensible, but in disease it is very sensitive.

Inflammation of the bone substance is called osteitis; of the periosteum, periostitis; of the marrow, osteomyelitis. The three forms of inflammation are difficult to separate. They are usually the result of external injury.

Inflammation of the bone has the same phenomena as inflammation occurring in soft tissue. It is attended by very many of the same changes, but on account of the dense substance making up the bone tissue somewhat different results come about. Serum, fibrin and pus may be found but they will be less in amount. The hard substance of the bone will be absorbed to greater or less extent and become replaced by animal matter, and there results a deficiency in the earthy or mineral matter. The newly formed tissue is the so-called granulation tissue of bone, which after a time becomes ossified. This tissue may be very vascular before ossification takes place. Although the bone is much enlarged as the result of the inflammation, yet it is much 

lighter. This condition is a rarifying process and it is called osteoporosis. The Haversian spaces are much increased in size and the bone is made porous. Osteoporosis may be observed both in spongy and compact bone.

Osteosclerosis is analogous to cicatricial formation of the fibrous connective tissue and is a result of inflammation of bone. It may occur without any apparent cause in senility in the diploe of the skull, causing it to become dense. It always occurs about carious and necrotic areas, constituting one of the chief means by which the sequestrum is surrounded by new dense bony tissue. Osteosclerotic exostoses of ivory-like hardness are called eburnations. Such bone is much heavier than normal.

Periostitis may be acute or chronic. In the acute form the morbid changes are found first in the periosteum itself, which becomes swollen, hyperæmic and more or less infiltrated with serum. In some cases infection takes place and suppuration occurs. The pus collects between the periosteum and the bone, elevating the membrane-(subperiosteal abscess). This frequently brings about a molecular destruction of the surface of the bone, causing the mineral structure to crumble away, and leaving softened and excavated areas, while particles of the disintegrated bone lie free in the surrounding tissue. To this molecular death and destruction of bone the name caries is given.

Caries usually occurs in cancellated bone. It is often seen in fistulous withers, caused by the pus burroughing down to the bone. The depressed surface has a peculiar velvety feel. It is also seen in poll-evil and may involve the axis or even the dentata in poll-evil. Foot Rot in sheep is often followed by caries of the digits. In treating such a condition, open up the sinuses or diseased parts and scrape out all the diseased portions and a little of the healthy part as well. Treat antiseptically and the bone will granulate the same as other tissues.

The acute forms terminate either in recovery without damage to the bone, when the abscesses are absorbed or rupture externally; or in caries or necrosis, with subsequent chronic disturbances induced by the process of bone-destruction, that may continue during the entire life of the animal or require surgical interference. 
Chronic periostitis is divided into fibrous and ossifying forms. In fibrous periostitis the characteristic feature is the transformation of the periosteum into a dense fibrous tissue, which closely adheres to the bone and later ossifies.

Osteitis and osteomyelitis are almost invariably associated. It is almost impossible to find the bone inflamed without the involvement of the marrow. This inflammation is infectious and seems to depend upon the bacteria which are disseminated by the blood and lodge in the vessels of the bone. The disease usually begins in the marrow cavities of the long bones, but may occur in the short bones, or even in the flat bones of the skull. The marrow is first found to be deeply congested and of darkred color. When the bone is sawed through the congested marrow bulges outward. In the majority of cases there is more or less suppuration and the pus collects in small cavities, forming abscesses in the bone. In severe cases extensive necrosis may result.

The greater number of cases heal by resolution, some in erosion of the bone, external evacuation of the pus, necrosis and then a chronic inflammation set up by the sequestrum. Some cases are fatal in consequence of pyemia. The extent of the necrosis that may follow osteomyelitis and periostitis is variable according to the severity of the case. In severe cases the entire shaft may die. The dead bone is known as a sequestrum. Chronic osteomyelitis results from the acute chiefly by the continued irritation by the sequestrum.

Necrosis of bone is death of the part, due to the nutrition being shut off. This is usually caused by injury to the periosteum. We see necrosis sometimes suddenly develop from external injuries, such as occur in the bars of the mouth from the bit. The bone dies and large pieces slough off-the sequestra. When the shaft of the large bone dies this way, it usually softens and liquifies and flows out through a fistulous opening. The discharge is ichorous. In the meantime new bone is forming under the periosteum. It is lacking in the characteristics of normal bone but it does very well.

The treatment for necrosis consists first in using slings unless the horse shows inclination to lie down. Soon as the pus 

can be located, open up and let it escape. If practical, remove all the dead bone. In necrosis of the bars of the mouth (the lower jaw, it is not always necessary to operate except where the parts are very sore and swollen. Then with a scalpel cut down to the bone and this will allow the escape of the pus, if any, and quicken the separation of the part if necrosed, and if not, it may prevent it. Always cut down through the periosteum; then let alone until the pieces come out. Do not force them out or use forceps. After they are removed, wash out with an antiseptic solution three or four times a day. The patients usually make a nice recovery. If the lesion is in the lower bars of the mouth, when the patient is getting well, use the common jointed bit or rubber covered chain. If the shafts of the long bones are affected, make a large independent opening by trephining; remove the sequestrum and wash out the cavity twice a day with antiseptics.

\section{ATROPHY OF BONE.}

Atrophy of bone is an interstitial absorption of the bony substance. This usually takes place in the cancellated portion of the bone, but it may occur in the compact bone, which then becomes softened and wastes away. It is not an inflammatory process although it may start as such because of some injury. In this condition the bone becomes diminished in size and weight and if it happens in the bicipital groove, it allows the patella to become dislocated very easily.

This condition is incurable, though rational internal treatment is in order,- - such as iron, arsenic, etc. The disease usually occurs in old animals.

\section{CONSTITUTIONAL OSTEOPOROSIS.}

Constitutional osteoporosis, or the big head, is a very peculiar disease because it is little understood. The pathological changes seem to be identical with those of localized osteoporosis. Some think that it is rheumatic, others that it is due to a parasite and consequently infectious. It is often found in 
breeding farms and affects large numbers of horses. The horse seems to be the only animal that is affected. It is more common in driving horses than in the running. It is thought to be due to a fungus on the grass or fodder.

In this condition we usually find the urine thick and mucilaginous; the horse tucked up in the flanks and inclined to lie down a good deal; back is arched; horse grows thin in flesh; but the appetite keeps good. The affected bone is probably painful and often the first sign is a peculiar lameness, which is intermitting and frequently locates across the loins. It cannot always be located and is often mistaken for rheumatism. The disease is not confined to the head alone, all the bones are affected, in fact if the bones of the head become affected, the rest of the body is usually affected also and the horse may break down all at once. This disease is much more common in the south than in the north.

Some claim to get good results by using iodide of potash and trephining into the enlargement and injecting antiseptics. This treatment does not seem to bring good results.' Hyposulphite of soda and calcium phosphate in equal parts three times a day is good. Give a complete change of diet and a change of location. In acute cases death may take place in two or three months. In autopsy we find the face bories so soft that you can sometimes run a probe through them. They are red and vascular, like granulating tissue.

\section{OSTEOMALACIA.}

Osteomalacia is an acquired disease of the bones of unknown origin. It is rather common in the human and bovines, but not so in other animals. In the human it usually affects adult individuals of the female sex, though it is often seen in males. It is thought to be caused by an innutritious diet, especially such as would occur among the poorer classes. Pregnancy, rheumatism, infection, intoxication, etc., have been blamed for its development. (See Merillat's, Vol. II.)

The disease is characterized by a soft, plastic condition of the bones, which depends upon the replacement of the original 

calcified osseous tissue with a new uncalcified osteoid tissue. The bones become subject to frequent fracture and increasing deformity, while the patient becomes more and more feeble and a profound cachexia comes on. Patient may die of exhaustion, but much more commonly succumbs to pneumonia or to some other intercurrent affection.

The bones usually retain their lamellar arrangement and their external and internal construction are alike histologically unchanged, except that the great mass of bone is decalcified and the altered bone appears homogeneous, sometimes fibrous. The decalcification usually begins at the periphery and extends in toward the center. Some of the lacunar cells remain after decalcification but many die. As the disease progresses, the osteoid substance melts away and increases the spaces between the columns of bony tissue.

The marrow is frequently congested and may be hemorrhagic or pigmented, from previous hemorrhages. The bones are so soft that they can be easily broken in two. Frequent fractures occur and serve to deform the bone. The ordinary form of the disease attacks the spinal column and thorax and spreads to the limbs and head. The most interesting and important changes occur in the pelvis, which yields to the pressure of the spinal column and the thighs. It becomes wholly transformed, making the bearing of young almost impossible.

Fragilitis ossium is a hardening of the bones to the extent of brittleness and usually occurs in old animals. It is due to the absorption of the animal matter and inflammation is usually the cause. In this condition fractures are very easy to occur.

\section{RACHITIS.}

Rachitis or rickets is a degenerate softening of the bones that occurs only in the young. It is due to some disturbance that prevents the ossification of the bone. It is really a constitutional disease and nutritional disorder. The lesion is characterized by deficient calcification and increased absorption of the bones, which in consequence are permanently altered in size and shape. All the parts of the skeleton are affected. The 
bones are usually shortened, thickened, rarified, curved, and twisted. The rarified condition is most frequently seen in the epiphyseal ends of the long bones, which become considerably enlarged.

This disease is not infrequently congenital, and seems at times to be hereditary. Bad hygiene, malnutrition, and certain inflammatory diseases seem to be exciting causes. In the congenital form the causes mentioned probably operate upon the young through the mother. 'The disease usually manifests itself in the first and second year of life. In cases that live beyond puberty the disease recovers but the deformities persist. Dentition is irregular and delayed. The animal, however, may be well up to two to six years and then be affected. It is common in puppies that are fed on starchy food. Foals, too, are apt to have it.

The first intimation of the disease is a peculiar lassitude and the play is not so lively; the long bones of the legs begin to bow, especially in the front legs, the convexity being outward. The femur is usually curved anteriorly as well as externally.

At the articular ends of the bones, where the diaphysis joins the epiphysis, instead of a narrow distinct white line, one finds an enlargement consisting of porous, irregularly formed bone in which there is a widely separated plexiform or fibrillar arrangement of osteoid substance deficient in lime-salts. These areas alternate with others of irregular ossification. The marrow is much like that found in the fetus. Next to the growing cartilage of the epiphysis is a zone of osteoid tissue in which the bony partitions inclose patches of cartilage.

The irregular growth and rarefaction of the bones give them a pronounced predisposition toward bending and fracture, especially of the green-stick variety, while the continued pressure produced by the weight of the body results in permanent deformities.

Treatment.-There should be a complete change of food. In the foal, wean him and feed on cow's milk liberally, say a gallon three times a day, and more as he grows older. Skim milk even if it is sour is better than fresh milk, because it contains less fatty matter; in addition give bone dust, oatmeal, etc., 
.

. 

but leave out corn and everything that tends to fat. In puppies change the food; give boiled meat with oatmeal, make a strong stew of it. If the legs in the foal do not get strong, apply splints and bandages. Lime water is good for rickets. Cod liver oil is good for puppies.

\section{HEALING OF BONE (下EPAIR).}

When a bone breaks, either completeiy or incompletely, there results more or less hemorrhage; the surrounding tissues are torn and infiltrated with blood; a moderate degree of inflammatory exudation and cellular migration takes place, but in the absence of infection the inflammation subsides after a few days, soon to be followed by regeneration. As early as the second day the cells of the periosteum begin to grow and proliferate and show many karyokinetic figures. In two or three days a vascular formative tissue has developed which is rapidly developed and differentiated into osteoid and chondroid tissue. This formative tissue produced by the peristeum is known as the external callus; that which extends in between the fragments is known as the intermediary callus. The tissue regenerating from the medulla is called the myelogenic callus.

The periosteal callus extends around the fragments like a capsule for some little distance on each side of the break. At the end of the first week the inner layers of the newly formed tissue have become differentiated into osteoid tissue and hyaline cartilage especially so in young and in animals like the dog, and then calcification sets in and porous bone develops-the bony callus. During the succeeding two or three weeks the amount of bony callus constantly increases.

The myelogenic callus is formed by the proliferation of the osteoblasts; it is not of so much importance as the periosteal callus. At about the end of the seventh week the periosteal callus is totally ossified, consisting of a porous, rather soft, osseous tissue, which gradually becomes substituted by new bone of considerable density by means of lacunar resorption and the formation of medullary spaces on the one hand (osteoclasts break down bone) and the production of new lamellæ or growth 
by apposition on the part of the osteoblasts on the other hand. In this way is produced a more solid dense bone-the definitive callus.

When great dislocation of the fragments has occurred, it may take several months or even years before the reparative processes are fully complete. In case of great dislocation the medullary canal is usually not restored.

Bones unite all right if given a chance. They are stronger at the point of fracture after healing than before. It is a popular idea that horse's bones will not unite well. Dogs, sheep and young steers take care of themselves and assist us but the horse never does, that is why we have such poor success in the healing of a horse's fracture. When the bones fail to keep in position they become intensely inflamed and this is kept up until gangrene sets in and ends the life of the animal.

\section{DISEASES OF THE NERVOUS SYSTEM.}

Diseases of the brain and nervous system are not as common in the lower animals as in the human. The lower animals are less liable to fatal results from shocks, as from fright or operations. These diseases are divided into three classes-(1) cerebral, when applied to the brain; (2) spinal, to the cord; (3) peripheral, to the nerves.

On account of the decussation of the nerves in the medulla, and in fact all along the spinal cord, injuries on one side of the brain produce paralysis on the other side of the body. The effects of sudden injuries to the brain are very noticeable, but slowly encroaching injuries are very puzzling in the effects which they produce. Any derangement of the nervous system has a tendency to produce either an increased functional activity or a depression, that is, hyperæsthesia or paralysis.

Cererral Congestion. - This disease is called vertigo, megrims, and sometimes blind staggers. It is due to a disturbance of the circulation resulting in a hyperæmic condition of the brain. It is more or less sudden in its attacks and it is usually due to pressure on the large veins of the neck by ill-fitting harness. It may be due to a plethoric condition of the animal and is 

associated with torpidity of the liver and indigestion, which results in paralysis of the pneumogastric nerve.

The horse will often stop, shake his head and let his ears droop; the muscles of the head and neck twitch, the eyes protrude, and he may become blind; his nostrils will be dilated and his breathing stertorous. He rears and plunges, loses co-ordination and goes down in a heap. The shock to the nervous system causes him to sweat, and while down he will often froth at the mouth.

While the animal is down dash cold water on his head and face. Take off his harness and give him plenty of air. As soon as the fit is over learn the causes of it and remove them if possible. If plethora is at the bottom of it, lessen his feed and give purgatives. These attacks often. come from stomach and liver troubles. Bleeding is useless.

Cerebral Anaemia.-This is a bloodless condition of the brain. There results a loss of motor and sensory power and the mucous membranes are pale. It may be due to rapid hæmorrhage. There will be great nervous prostration from weakness and debility. The treatment should be rational; treat the general symptoms to supply the body with nutrition.

Cerebral Embolus.-In this condition some of the blood vessels of the brain become plugged by a clot and embolism results. Paralysis of all parts of the brain supplied by these occurs. The clot is usually formed by reason of weak cardiac power or valvular insufficiency. The embolism causes softening of the brain. The symptoms are stupor, weak mindedness and pale mucous membranes.

Apoplexy.-As applied to the brain this disease means cerebral hemorrhage. It is characterized by a sudden loss of both motor and sensory power. The hemorrhage may occur in one of three ways: first, in rupture of the blood vessels of the brain; second, from the filling of the ventricles with blood; and third, from a hemorrhage into the arachnoid space. The first and third ways usually prevail in the horse.

The disease comes on suddenly always: the horse falls to the ground, froths at the mouth and the special senses are suspended,- - he cannot hear, taste, smell or see. The mucous mem- 
branes are livid and the breathing more or less stertorous. If the case is severe death will result in from a few hours to eight or ten days. If not severe, the clot may become absorbed. The horse seldom fully recovers.

It is not considered safe to bleed an animal in this condition. Elevate the head, pack it in ice and keep the rest of the body warm by clothing and friction. Give an enema and inhalations of ammonia, hypodermic injections of alcohol, atropine, etc. Never give medicine by the mouth in such a case, because the function of deglutition is suspended.

Cerebral Meningitis.-This disease is inflammation of the coverings of the brain. Cerebritis is inflammation of the brain substance. It is not possible to draw the line between the two as in the human. The condition exists in two forms, acute and chronic. The meningitis is usually due to idiopathic or traumatic influences. The idiopathic influences are exposure, exhaustion, sunstroke, etc. The traumatic include wounds, concussions, etc., from violence.

Semeiology.-There is marked congestion of the visible mucous membranes, especially those of the eye. There is muscular twitching and excitement of the animal; the twitching sometimes amounts to spasm of the limb or of two limbs. These spasms sometimes involve the whole body, producing cerebral convulsions. The period of excitement is usually followed by one of depression, and then an abnormal activity. In some cases it amounts to stupor or coma; but in meningitis this stupor period is comparatively short and the excitement severe. There is a well-marked constitutional disturbance until the animal becomes delirious and he tries to climb over the manger. This desire to climb comes on during the first twenty-four hours. Convulsions may come on at the same time and throw him down, but he gets up and goes on climbing again. During the stupor he will hang his head and seems almost inclined to fall.

The symptoms of meningitis are more violent than those of cerebritis. In cerebritis there is less fever and less excitement and the periods of stupor are more complete and longer continued. In cerebritis the animal will stand a great part of his time with his head against the wall or manger; his appetite is 
usually lost, partly due to fever and partly due to the loss of sense. After a time, both in cerebritis and meningitis, the animal will lose his power of co-ordination, does down and is unable to get up again; he will lie prone and keep his legs going. Put your finger in the eye or ear and no resistance is offered. Death usually takes place in two or three days.

Cerebral meningitis runs a very rapid course compared w:th the human, terminating in the lower animals sometimes as quickly as the end of the first day. The differential diagnosis between it and blind staggers is quite simple: in blind staggers there is no fever, no congestion of the mucous membranes and the eyes are not bloodshot, while the very opposite occurs in mad staggers or cerebral meningitis, the bloodshot eyes being a special characteristic. Blind staggers usually yields to treatment while mad staggers does not.

Treatment.-Purge the animal with aloes and give diuretics - potassium salts. Allay the nervous irritability with gelsemium, aconite, belladonna or acetanilid. Belladonna is particularly indicated on account of its contracting the arterioles. An ice poultice to the head is good, but the horse is dangerous to handle so do not get caught in the stall in trying to apply the ice. It is very difficult to do much for the horse in this disease and recoveries are rare. In case of recovery give the animal three or four months' rest at pasture.

It is difficult to diagnose this disease with any degree of certainty. At autopsy the meninges are thickened, with adhesions here and there. You will find coagulated lymph in the ventricles, also in the sulci and other parts of the brain.

Cerebral softening.-In this disease there is a softening of the brain substance, which is altered in color. It is sometimes quite liquid and is softened always, especially in spots. It looks like abscesses and the color varies from dirty pink to white. Many cases have been seen where the cerebrum looked like pus. Cerebral softening is known among horsemen as a "dummy." Such cases are quite common. It is hard to say what is the cause, but we think that the condition comes from acute or subacute attacks of cerebritis or from interrupted circulation in the brain. In the human it is caused by long-continued use of alco- 
holic drinks. The disease is in reality nothing more than cerebral degeneration.

The symptoms are very marked but require close observation to detect them. The horse is more or less stupid and seems to lack ambition and often staggers. Mild excitement will arouse him for a time and you must look out for this in examining a horse for soundness. The animal looks stupid, will stub his toes after being backed out of the stall. His ears may be moving back and forth and his face lacks expression. To tell a dummy just observe him as he backs out of a stall or turns around in it. He lacks complete power of co-ordination. Staggers and wabbles a little. Cross his feet and they will stay there. Put your finger in his ear and he will not resist. The treatment is useless. The real "dummy," is due to cerebral softening or chronic dropsy of the lateral ventricles.

Myelitis.-This disease is inflammation of the spinal cord. The meninges are usually involved. The causes are violence in the form of external injuries, disease of the bones of the vertebræ, and exposure to dampness and extremes of temperature. It often comes on without any appreciable cause.

Semeiology.-When the meninges of the cord are first affected, the first symptoms will be tonic spasms of the limbs, usually the hind ones. This is probably because it affects the loins first and then extends forward. The spasms are often so severe as to jerk the horse off his feet and throw him down. He stamps spasmodically and involuntarily. If the cord itself is first affected there is an absence of these spasms and instead there is a loss of motor power. You will notice that the hocks dip into each other, the toes will turn out, the gait will be wabbly,-no spasm and no delirium. The motor paralysis increases so that finally the animal cannot move without falling; if he does not move, he will stand braced from twenty-four to fortyeight hours. While lying his expression is haggard and he looks in great distress.

In chronic spinal meningitis the animal is very excitable, the least bit of excitement affecting him. $\mathrm{He}$ will also manifest nervous twitching.

PARESIS.-This disease is ordinarily spoken of as paralysis 

or it may be called palsy. It is a loss of the motor power as concerning the voluntary movements of the body: It is a symptom of other diseases. Paraplegia is the most common form of the paralysis,-meaning paralysis of the body transversely.

The causes are centric or reflex. Centric is due to external violence, perhaps severing of the cord, or anything that will produce pressure on the cord, as fracture of the vertebræ. "Reflex causes must be referred to the periphery of the body, from whence a disturbance is carried to the nerve centers. We see this in canine distemper and in metritis in all females. The reflex form of paraplegia resists treatment very much. 'It is likely to occur from indigestion; impaction of the rumen in cattle may cause it.

The symptoms show a partial or complete loss of power, first the motor. If the cause is reflex, there is a loss also of the sensory. Constipation is usually present; the muscles around the neck of the bladder are relaxed and the urine dribbles away. Prick the hind parts of the horse and he will not show any pain. If this is done in the dog he will cry out. This shows that the motor nerves are affected before the sensory.

Hemiplegia is paralysis of one whole side of the body. The cerebrum is usually affected. A ruptured blood vessel may be the cause. Recovery is rare and also the disease itself in the lower animals.

Treatment.-Remove the cause. If impaction is at the bottom, then give such drugs that will loosen the bowels. Dogs usually recover from paraplegia. Bitches usually die. Give the animal rectal injections. Give diuretics and stimulants such as potassium, iodide and nux vomica.

Hydrocephalus.-This is rare, especially in the adult. . It is seen in the fetus and renders parturition difficult. The symptoms are very similar to those of cerebritis, but more mild in character. The animal is stupid, keeps getting more so until coma sets in and he dies. The effusion is usually in the subarachnoid space or in the lateral ventricles.

In the early stages of hydrocephalus there is fever and a lit. tle irritability. The stupor and insensibility keep on increasing 
with occasional epileptic fits until the disease terminates in coma, then convulsions and death.

Pathology.-In autopsy, on opening the cranial cavity we notice that the brain is altered in form; the fissure between the hemispheres is almost obliterated. In cutting down through the corpus callosum you find water in the ventricles, varying in quaritity from 1 to 8 ounces. Upon emptying the water out we find the septum lucidum broken down, making one cavity. The arachnoid is thickened and opaque, particularly over the cerebellum and it is often studded with spots of inflammation. The fluid in the ventricles is usually pale, thin and watery, but sometimes quite turbid. Effusion may take place into the lateral ventricles, or into the subarachnoid space. A horse so affected in the chronic form is a dummy.

The treatment is not very satisfactory. In mild cases the disease may give way to iodide of potash, iron, vegetable bitters, and mild counter-irritants externally. Keep the animal quiet; give soft diet and rest.

\section{TABES DORSALIS.}

Tabes dorsalis or locomotor ataxia, is the same as sclerosis of the cord. It is sometimes called "crick back." In the domestic animals the disease affects the white matter of the cord. In the human the gray matter may also become involved. This condition is a contraction and hardening of the columns of the cord. In the horse it is usually the supralateral columns. The inferolateral may also become affected. The loins are about the first to become affected, but the disease may start in the cerebellum and travel down the cord. The motor columns are affected more than the sensory.

Etiology.-The disease usually develops as the result of exposure to cold and storms, especially if the horse is not in good condition. It may occur in old horses and debilitated ones. The minute vessels of the cord are diminished in size and the gray matter can be easily ruptured. In cases of syphilis in horses this condition is always present.

Semeiology.-Tabes dorsalis comes on slowly. We notice 

first an unsteady gait behind, especially if the animal turns quickly. If the disease originates in the cord, then the lesion seems to be confined to the hind limbs; if in the cerebellum, then the animal is wabbly both before and behind. The eyes squint, which is particular evidence of sclerosis of the cerebellum. If the lesion is in the cerebellum the animal will walk in a circle and the more severe the lesion, the smaller the circle he will walk in. The paralysis increases continually and usually the symptoms are increased also. In an acute case the muscles of the hind parts waste. The inclination to throw the weight upon the fore limbs on account of the increasing paralysis causes the muscles of the hind limbs to be inactive and therefore to waste away.

In the horse we have no evidence of acute pain; in the human it is said that there are pains in the legs at times.

Treatment.-There is no treatment that will cure the disease and the prognosis must therefore be unfavorable. If you give strychnine, electricity, bromine, iodine, iron or arsenic and liberal diet with gentle exercise, this course of treatment will prolong life.

\section{TETANUS.}

Tetanus is a continuous tonic spasm of the muscles of the body. Usually the voluntary muscles are the ones involved, but the involuntary muscles may be involved also.

Etiology.-The bacillus Nicolaier is the specific cause. This germ was discovered in 1884 . Nicolaier found the germ in the earth and introduced it into animals and produced tetanus. The germ is drumstick-shaped carrying its spore in the big end. It lives in the dirt everywhere and it is anærobic. For this reason the disease will result from small wounds which easily close up more readily than large wounds that are open and exposed to the air. The germ will not grow where there is free access of oxygen. More than one-half of the tetanus cases come from small nail pricks that hardly lame the animal. All an:mals are subject to it but the horse, perhaps more than others. Different parts of the body are affected. When the whole body is affected 
it is called OrтнотоNos. When the masseter muscles are affected it is called Trismus. Both forms are common. The tetanus germ must be associated with other germs in order to set up its infection; it cannot work alone.

Semeiology.-The symptoms are clearly diagnostic usually. The first thing you will notice is a contraction of the massetcr muscles. The muscles will be drawn around the mouth. The animal seems to have difficulty in eating and he secretcs an enormous amount of saliva. After a few hours he becomes excitable, the nose is extended, the facial muscles twitch and any little disturbance excites the animal. The eyeballs are retracted in the orbit and look small. The membrana nictitans is liable to be raised constantly over more or less of the eyeball, and when the head is raised will cover it. An intelligent horseman will notice the beginning stiffness of gait. Trismus is usually well marked; after twenty-four or forty-eight hours the development seems to be quite gradual, other times violent, in which case the animal will soon die. The spasms which were at first in the masseters finally affect the whole body. The dorsal, cervical and gluteal muscles are the most affected. The tail is elevated and it maintains that position and trembles. The respiration is accelerated and the nostrils are dilated; the ears stand like sticks, the limbs are stiff and are straddled; locomotion is very difficult; flanks are tucked up; ribs are tightly drawn, due to contraction of the intercostals. As the pain becomes intense, the animal sweats profusely and is very excitable. The spasms come on from time to time and between times the muscles relax a little, but not enough to cause much hope. The action is spasmodic. During a spasm the gluteal muscles often shoot out behind like a goose in flight, and the action throws the animal to the floor and he cannot rise until the muscles relax. An animal usually does not rise after going down on account of the stiffness of the legs; he is unable to get them under him enough to get up. The recumbent position and being unable to rise increase the frequency and severity of the spasms. The spasms are easily brought on, - a rat or loud talking or the presence of a stranger may be the cause. The saliva is ropy, the pulse grows hard; the 


appetite remains good but the bowels are inactive. The peristaltic action is interfered with.

As a rule the horse persistently stands and if he can get up after being down, it is a favorable sign. Death comes from two causes,-syncope and asphyxia. The muscles of the throat contract and cause the asphyxia. Death occurs in from two to ten days.

Prognosis.-When a spasm throws a horse down, there is little hope. If the animal can eat and the trismus is slight and the respiration is not affected then the results may not be so bad. We find by experience that the mortality runs from $75 \%$ to $80 \%$. If the animal lives thirteen days he has $50 \%$ of the chances to recover. If he lives eighteen days he has $75 \%$ in his favor. If he lives twenty-one days he has $95 \%$ in his favor. Dr. Baker says one of his cases died on the twenty-eighth day.

Treatment.-There is no specific treatment. Give a rational course toward allaying the symptoms, bearing in mind that if the animal lives two weeks he will probably recover. Pay attention to the hygiene. If it is cold, clothe him warmly; if it is hot, keep the animal cool. Keep away exciting conditions. Give linseed gruel-about two gallons, and keep this before him all the time. Give him nothing else to drink. Let the horse have as much of the gruel as he will drink. The gruel tends to loosen the bowels. Purgatives are useless. It is a good plan to put the horse in a sling. Bear in mind that he cannot recover on his side.

Give drugs that will quiet the nervous system, such as belladonna, prussic acid, morphine, ether, chloral hydrate, lobelia and gelsemium. If the tetanus antitoxin can be given in time it will cure the disease, but it must be given early. Some claim that this is useless, but it has not proven so in my experience. Give about $40 \mathrm{cc}$ for the first day than $20 \mathrm{cc}$ the second; the third day the same. If on the fourth day the animal is holding his own, then give another dose of $20 \mathrm{cc}$. Keep this up for ten days. If however the animal begins to grow worse, then drop the serum treatment. 
The following is a good prescription:

$\mathbf{R}$

Gelsemium Fl. Ex................ ounce

Lobelia Fl. Ex.................. ounce

Belladonna Fl. Ex................. ounce

Aqua ad qs....................... ounces

Give one ounce every 3-4 hours.

If possible give per mouth; but if there is much trismus, dilute it and give per rectum. If administered this way let it be about blood heat. A hypodermic of 4 grains of morphine three or four times a day is good with this prescription.

The post mortem shows very few lesions.

\section{CHOREA.}

Chorea is convulsions of the voluntary muscles and it is most common in dogs. In the dog it occurs oftener in the legs while in the horse it is seen most often in the neck and hind quarters. In the horse it seems to arise as an original lesion while in the dog and in the human it usually follows some other disease. In the horse it may come on suddenly from indigestion, particularly that which arises from over-feeding. In dogs and children it may occur from worms in the intestines. In all these cases there is an increased irritability of the nerves. In the dog it is often seen in a fatal form as a complication in distemper.

Semeiology.-The disease comes on suddenly, especially in a case of distemper in the dog. The disease often attacks a foreleg which will be lame for a day or two before any jerking begins. If you make the animal step on the affected leg by taking the other up, he will go down and when there is jerking in it he cannot bear any weight upon it at all. The condition rapidly spreads to the whole body and the animal finally dies from exhaustion. During these attacks the dog will whine, particularly if alone. There probably is not much acute pain, only general distress. The jerking may be continuous night and day. Eventually the animal becomes emaciated, especially the dog.

Some think that the disease is transmissible, so much so that animals thus affected are condemned for breeding purposes. At any rate the offspring of choreic stallions are liable to have the disease. Horsemen call it shivers. In the stall the horse will 

show his trouble by putting his leg out to one side in a peculiar manner when stepping over. In backing out of the stall, he may take a few steps all right and then be unable to go further. The tail may shiver as well as other parts. In severe cases in horses spasms severe enough to throw them to the floor come on occasionally. Some horses with this disease are unable to back at all, but can go ahead. Chorea is liable to become chronic and all chronic cases of this disease are without exception incurable.

There is no special lesion revealed by the post mortem, except that in long-standing cases we find thickening of the arachnoid, effusion into the arachnoid space and hardening of the outer layer of the cord. In acute cases in the dog the medulla show softening.

Treatment.-Chorea is very unsatisfactory to treat. In severe cases it is incurable. Constitutional tonic treatment is about all that can be done for the animal. Give a liberal diet, correct the hygiene and avoid fatigue. For drugs give iron, zinc, copper and in some cases small doses of strychnine. You can combine with these cinchona and gentian. In case of whining dogs you can relieve the distress by giving valerian fluid extract with a mineral tonic.

Give Fowler's solution in gradually increasing doses till the maximum dose is reached, then reduce the dose gradually to the minimum, then up again, and so on for two to four weeks. Nitrate of silver in bread pills is very good; give for a week and alternate with sulphate of copper. Recoveries are rare.

\section{DISEASES OF THE REPRODUCTIVE SYSTEM.}

The diseases of the reproductive system are not so common in the lower animals as in the human. There are only two specific diseases, the malignant and simple pox.

\section{SIMPLE POX.}

In its nature simply pox is a phlyctenoid eruption. The eruptions occur on the vulva and extend into the vagina. It is never seen in the sheep, pig or dog. It is common in the human 
and in the mare. The eruption is a simple blister called a phlyctena. This occurs in the male sometimes on the body of the penis. The period of inoculation is from 24 hours to 3 days, The lips of the vulva swell as large as the wrist. . In the stallion the sheath swells. The disease is transmissible from one animal to another by coition. No special constitutional disturbance is produced. The disease is self-limiting and if copulation ceases recovery will take place in 5 to 10 days.

Treatment.-Give cool laxatives and mild stimulants. For a local application use sulphate of zinc ( 5 grains) to the ounce of water. Bathe the parts three times a day. This disease is also known as coital exanthema.

\section{MALIGNANT $\cdot$ POX.}

Malignant pox is known as dourine or equine syphilis. The disease was brought to this country from France. In 1817 it was known in Prussia; in 1821 in Hungary; in 1836 in Italy. It has never been known in Belgium or England. The special pathology of this disease was gotten from Tanhoffer.

Etiology.-The trypanosoma equiperdum is the specific parasite causing the disease: It is a single-celled animal parasite. This parasite was discovered in South Africa in connection with the tsetse fly disease and sleeping sickness. The lesions resemble syphilis in the human, but the two are different. Syphilis is transmissible from parent to off-spring in the human but dourine is not in the horse. A syphilitic stallion cannot impregnate a mare. If a syphilitic mare should conceive she will abort or else the foal will be born dead.

Semeiology.-The elevated plaques on the body and pustules on the penis and vulva are the first noticeable indications of the disease. These ulcerate. They may occur on the glans penis and are accompanied by the swelling of the sheath. In the mare the vulva swells, and turns out, exposing the clitoris to view. The appetite is poor and the animal becomes lazy. If the disease occurs in an acute form, it will kill; if it is chronic, in the course of a month or so the animal begins to be wabbly in his hind parts. Sometimes the ulcers heal and are replaced by others. After a 

month or so depigmentation takes place in the skin,-on the inside of the thighs, scrotum, vulva, and elbow. The depigmented patches are about the size of a quarter to the size of a man's hand and turn white. The depigmentation is a progressive process. The hair gets shabby and the animal wabbles more and more. As weakness develops the legs swell and in 12 to 16 weeks a profuse muco-purulent discharge takes place from the nose. The scrotum and testicles may be very large.

In the mare the mammary glands swell and the clitoris and mucous tissue are protruded. The mucous membrane of the clitoris is of yellowish color. The subacute runs into the chronic;

Although some animals appear to recover they should be retired from breeding, because it always revives the disease.

The only peculiar morbid anatomy is in the spinal cord, the locomotor ataxia is the same as that of other diseases.

Treatment.-Dourine is a contagious disease; therefore destroy all the animals that are affected. If necessary quarantine them. The old quarantine laws allowed the animals to go back into the stud after three years, but it was found that they gained no results, for stud work revived the disease.

\section{URETHRITIS.}

In stallions this condition comes always from serving mares that are affected with leucorrhoea. It is characterized by pus, but no germ has been isolated as has been done in the human.

Micturition is painful.

Retire the animal for a few days. Give a purgative and saw palmetto. Use the zinc sulphate solution two grains to an ounce of water as an injection twice a day.

Bull BuRnt is similar to urethritis but it affects the còvering of the penis rather than the uretha. It is usually the result of too frequent copulation. Inject into the sheath a solution of acetate of lead-half an ounce to the pint of water. Manipulate the part and repeat night and morning.

BURNT Dog is a foulness of the sheath from the accumulation of natural secretion in the sheath, which produces a catarrhal discharge. Use the same treatment as for the preceding. 


\section{PHIMOSIS.}

Phimosis is the inability of the animal to extend the penis. This may be due to congenital malformation. It may be due to wicer or to some other interference, as edema of the sheath.

This condition can be recognized by the foulness of the sheath in which the animal urinates.

Treatment.-In congenital cases cast the animal if necessary and insert a probe-pointed bistoury and cut down the prepuse about half an inch to one inch. Bring the organ down and wash off the parts: The sheath should be cleaned out night and morning until the wound has begun to granulate. Then use the white solution.

Horses may get the notion that they cannot let the penis down and consequently urinate in the sheath. Put a twitch on the animal and bring the penis down once a day for three or four days.

\section{PARAPHIMOSIS.}

Paraphimosis is the inability on the part of an animal to retract the penis. There may be a swelling of the sheath and the penis itself may be swollen. Following castration we find a typical example of this disease, probably on account of the swelling of the penis and sheath. The penis swells mostly on the dorsal side and the covering becomes tense and glistening. Such a severe case is usually due to local irritation. Paralysis of the penis may be the cause. In the dog sometimes following copulation the erection cannot subside and the organ becomes gangrenous in the course of three or four days.

Treatment.-If the condition comes from castration, you will have to reduce the swelling. Give diuretics internally and plenty of exercise and fresh water. If it comes from injury and inflammation has set in, put on ice pack tight upon the horse's hody near the penis. Keep this up until the swelling goes down. Give diuretics and laxatives. Sometimes we find that the penis is cold and cyanotic; scarify it from six to a dozen cuts and foment it with tepid water. This will produce a dilation of the 

vessels and consequent hemorrhage. In case of paralysis try the cold water treatment by throwing very cold water upon the retractor muscles. Do this twice a day. The electric battery can be applied here and strychnine internally. After putting the penis in place, then put a bandage around the body to hold the wet packs in place. Keep the penis suspended tightly up against the belly with a broad bandage passed around the horse's body.

In the dog you will find that the penis is purple and congested.. Scarify it and bathe the parts with water. If it cannot be replaced in the sheath cut down the prepuce a little. If you cannot do better, amputate.

\section{DROPSY OF THE OVARIES.}

Dropsy of the ovaries follows congestion and large cysts sometimes result. The condition is common in old cows and in the human, but not in the other animals. When a chronic congestion occurs as in this condition, the animal is always in heat. But it is an unnatural condition, and the animal will not breed. Such animals seem to change their form in time and become more masculine in looks. They do not make good breeders or good feeders and the best thing is to spay them. The cystic ovary can be crushed and the fluid allowed to escape into the abdominal cavity.

\section{HYDROMETRA.}

Hydrometra is dropsy of the womb. The mixture that fills the womb is a mixture of serum and pus. It is the result of long-continued inflammation. It may follow parturition and it may occur without any assignable cause.

If you are sure of your diagnosis open the os uteri with the hand and empty the womb. Wash out with boracic acid, or dilute coal tar emulsion. After a week or two, change to sulphate of zinc. Give a tonic of iron and alcohol. 


\section{NYMPHOMANIA.}

This is a condition where the mare is always in heat. The clitoris is in a state of chronic congestion. Excess of passion in the male is called satyriasis, but the disease is more common in the human than among the lower animals. Females in this condition are in heat all the time, but as a rule they do not breed. The disease is more common in the cow than in other females and it is due to luxurious living. Sometimes it may be caused by cancer of the clitoris and in such cases it should be amputated. Males should be put to harder work and the hygiene attended to. Females should be spayed.

\section{HYSTERIÁ.}

Hysteria is an excessive nervous condition seen in females only. The whole body is in a hyperæsthetic condition. It usually comes on with the period of œestrum. The genital organs are congested and in the attacks that follow the symptoms are similar to those of tetanus. The spasms may throw the animal off her feet and are manifested by kicking, biting, etc. The temperature runs high, sometimes to $112^{\circ} \mathrm{F}$. Try bleeding the animal. If this does not work give gelsemium and bromide of potash. Give a purgative and keep the animal quiet.

\section{LEUCORRHOEA.}

Leucorrhoea, is a muco-purulent discharge from the vagina and uterus. The discharge is whitish in color and in most cases it is caused by a retained placenta. The mare is usually unthrifty, becomes thin in flesh, has a staring coat and a more or less purulent discharge. The trouble may run on for years if not arrested.

Wash out the uterus with half of one per cent of liquor cresolis compositus U. S. P. once a day for a week, then twice a week for a week or two. If ulceration is present treat the sore places with pure boracic acid in the powdered state, and 

keep this up for about two weeks. Give iron, arsenic and vegetable bitters.

\section{ONANISM.}

The word "onanism" mean alone. It is masturbation on the part of the animal. The act becomes a habit and leads to general debility. It produces softening of the brain and will eventually cause death. Horses are very subject to it and the habit ruins young studs. The offspring of such an animal will be weak and inferior. The horse should be watched and exercised. Attend to the hygiene. Various mechanical appliances are used to prevent the acts of self abuse, and when these fail castration is the only remedy.

\section{DISEASES OF THE ORGANS OF SPECIAL SENSE.}

\section{SIMPLE OPHTHALMIA.}

Simple ophthalmia is inflammation of the conjunctiva. It is usually the result of some foreign body being introduced into the eye or the extension of some inflammation through the lachrymal ducts in catarrh or glanders. It is often caused by facing a cold wind in long drives or from caustic substances getting into the eye.

The eyelids are more or less swollen and the extreme sensitiveness of the eye causes the animal to keep the eyelids shut. There is more or less pain and a copious discharge of tears. The cornea becomes inflamed if the condition lasts long and it becomes opaque.

Examine the eye for a foreign body and if found remove it. This is best done with a pair of curved forceps holding the curved side toward the eye so as not to injure it. Foreign bodies become embedded in the mucous membrane, which closes around them, so look carefully. You will recognize the presence of one by the local swelling where the body is and you can often as it were break down the mucous membrane and get at it by curretting the most prominent point. 
After removing the object, treat with anodyne and mucilaginous substances. Common salt and distilled water are good to allay the inflammation. Use one-half dram to the pint of water. The white of an egg introduced into the eye is also good-it lubricates the surface and relieves the irritation. Linseed tea or a grain of linseed introduced into the eye is good. In a very bad case keep the animal in the dark and apply cocaine occasionally.

\section{FUNGUS HAEMATOIDES.}

This is a bleeding fungus. It is a medullary soft cancer often seen in cattle. It usually starts in small purplish spots on the conjunctiva. It is malignant and spreads through the eye, often working back to and through the bone. The putrefactive matter of the ulceration causes cachexia and the animal may die from the absorption of the products of cytolysis.

The only hope is to remove the growth in the early stage. If the eye is involved remove it and cauterize. When the granulations fill up the eye about one-half, use the white lotion to prevent further growth. The animal should be sent to the abbatoir on account of the liability of recurrence.

\section{ENTROPIUM.}

Entropium is the turning in of one eyelid or both. They bring the eyelashes in contact with the eye and irritate it. The cornea is more or less cloudy and there is a discharge of mucopurulent matter from the eye, giving the animal a disagreeable appearance.

The treatment is surgical and involves the removal of an eliptical section from the eyelids, thus shortening them up. The result of the operation as a rule is pleasing. The condition is very common in dogs. 




\section{ECTROPIUM.}

In this condition the eyelids are too short; they turn the lashes out and expose the conjunctiva. This is not so painful as in entropium, but it is unsightly.

In mild cases we may use astringents such as sulphate of zinc (2 grains to the ounce), or a saturate solution of borax, or cold tea. Apply the solution two or three times a day. If this fails we sometimes sew the lids together the same as in dislocation of the eye. We may be required to cut out an eliptical portion, but this is rare. If the zinc lotion proves too weak, then increase its strength to 5 grains to the ounce.

\section{LEUCOMA.}

Leucoma is an opaque cornea. The cornea is cloudy. This condition may be produced by blows, which however, are not severe enough to break the cornea, but which often produce a permanent cloudiness. The more dense and organized the exudate, the more permanent will be the opacity; and the whiter it is, the more likely is it to be incurable. While it is blue (pale blue) there is hope of recovery; but as it becomes white, the chance diminishes. Sometimes there is a white patch on the cornea at the point of injury. This will remain permanent, but if the contusion is mild, the exudate will become absorbed. For local treatment apply warm fomentations to the eye and give anodynes. Later use local applications of saline or borated water. Give stimulants. In three or four days change to distilled or fluid extract of witch hazel-the distilled can be used clear, while the extract should be diluted 75 per cent. ' Follow this with sulphate of zinc or silver nitrate- 5 grains to the ounce. In old cases use stronger solutions-say 10 grains to the ounce. Always use distilled water when treating the eye.

\section{ULCERATION OF THE CORNEA.}

This is liable to occur in connection with any wound, but it is often found in distemper in dogs. The eyelids become glued 
together and pus accumulates inside them and irritates the cornea. The ulceration may occur in two or three places in the cornea, and then the sores become confluent. Unless the lids are softened and the pus let out, the cornea may be eaten through and the aqueous humor escape.

Remove the cause; reduce the conjunctivitis and touch the ulcer lightly with lunar caustic once a day. In case the cornea is not ruptured, granulations may form in patches on its surface and these will be red as.beefsteak. In such cases cauterize every three days with lunar caustic.

\section{INFECTIOUS KERATITIS OF CATTLE.}

Keratitis is inflammation of the cornea. It is a constitutional disease and one especially peculiar to cattle. It is very common and often runs through a whole herd. This would indicate that we have to deal with a specific germ but as yet none has been found. The disease runs a regular course and it is usually benign.

Semeiology.-Tears runs down the cheek... The eyelids are more or less swollen and the cornea is often ulcerated.: Acute cellulitis is also present. The cornea is opaque. Recovery may take place in 4-12 days, but in some cases the cornea may become ruptured, the aqueous humor escape and the eye becomes destroyed.

Treatment.-Keep the patient in the dark. Apply warm fomentations -with salt water. Do not let the iris remain in one position too long a time. Inject a little atropine to 'dilate' the pupil and if it does not contract in a few days then use calabar bean. After the inflammation has run its course use sulphate of zinc lotion. Give laxatives and soft food.

\section{FILARIA OCULI.}

The filaria is a little thread-like worm which is sometimes found in the anterior chamber of the eye. This is especially seen in Canada. It is not known how the parasite gets into the eye, but probably through smaller worms which develop in the 


blood. The worm is $1 / 2^{-1}$ inch long and is as slender as 60 cotton thread. Sometimes it lies quietly and sometimes it flashes about in the humor. This movement stimulates the glands to secrete more humor and the eye bulges out, causing great distress.

Treatment.-In case of the horse, lay the animal on his back, turn the head on the poll and fasten the upper eyelid back with a few stitches. Do not use a speculum to hold the lids in place. First apply a little cocaine; some use chloroform, but I do not think that this is necessary. With an ordinary scalpel cut into the cornea on the upper side of the eye. Make an incision about an eighth of an inch long and then press out the humor. The parasite will usually come out with the humor, but if not, press it out. You may have to take it out with a pair of forceps. This operation is called the Sclero-corneal operation. The wound will heal by first intention, the humor will be renewed and the eye will be all right in a few days. Never make the incision on the lower side of the eye, else the humor will run out as fast as secreted and form a fistula. Before operating pass the blade of the scalpel through a flame to insure that it is sterile.

\section{AMAUROSIS.}

This condition is paralysis of the optic nerve. It is sometimes called Gutta Serena or Glass Eye. The eye is insensible and there is total blindness. It is common in people and horses and rare in cattle and dogs. The causes of the affection consist of tumors and other diseases of the brain implicating the optic nerve. Injury to the nerve between the brain and the eye and inflammation of the roots and also the endings of the nerve or undue pressure upon the same from inflammatory effusion are etiological factors. Optic palsy may also occur from an overloaded stomach, from a profuse hemorrhage, and even from pressure of the gravid womb in gestation.

Semeiology. - Wide dilatation of the pupis is a marked symptom. The whole interior of the eyeball is exposed and the expansion remains the same in light and darkness. The horse does not swerve when a feint to strike is made unless the hand causes a current of air to come against the face. The ears are held 
erect and turn quickly at any noise. The animal steps high to avoid stumbling.

Treatment.-Treatment is only useful when the disease is symptomatic of some removable cause, such for instance as congested brain, an overloaded stomach or gravid womb. When recovery does not follow the termination of these conditions, apply a blister behind the ear and give dram doses of nux vomica three times daily.

\section{DETACHMENT OF THE RETINA.}

This occurs in case of hemorrhage between the chorid and the retina. It produces a loss of functional activity of the retina and may run into amaurosis. The cause of this condition may be a severe blow on the head, a fit of coughing, or rapid, excessive hemorrhage. It is common in people and horses and dogs but is never seen in cattle. It frequently occurs in horses in connection with castration, cuts from barbed wire fences, etc. This affection can sometimes be cured; amaurosis seldom.

In treating such cases give tincture of iron and nux vomica internally. Inject a few drops of witch hazel (distilled extract) into the eye three times a day.

\section{STAPHYLOMA.}

This consists of a bulging forward of the cornea at a given point by the sacular yielding and distention of its coats. The tumor may be transparent or opaque. The disease is common in the human and dogs but rare in horses and cattle. In the transparent form the tumor is bulging, distended and cyst-like; but in the opaque the tumor is thick and is formed like a grape. The bulging kind is more common and it grows larger than the solid tumor.

If the bulging kind is detected soon enough, puncture it and liberate some of the aqueous humor. This may save the eye. If the condition becomes chronic and a thickened mass is found on the outside of the cornea, it must be removed. This operation destroys the sight and the cornea cannot be replaced. A glass 

or rubber eye can be inserted for the sake of appearance. The wound should heal with little difficulty. Be sure that the instruments are sterile before operating.

\section{PERIODIC OPHTHALMIA.}

Other names for this condition are Specific Ophthalmia, Recurrent Ophthalmia or Moonblindness. It is an inflammatory affection of the interior of the eye, intimately related to certain soils, climates and systems, showing a strong tendency to recur again and again, and usually ending in blindness from cataract or other serious injury. It is peculiar to the horse.

Semeiology.-The local symptoms are in the main those of external ophthalmia, with, in many cases an increased hardness of the eyeball from effusion into its cavity. The contracted pupil does not contract much in darkness nor even under the action of belladonna. The opacity advances from the margin of the cornea over its whole surface. So long as it is transparent there can be seen a turbid aqueous humor. This may or may not contain floculi. The dingy iris is robbed of its clear black aspect, the lens is cloudy and there is a greenish yellow reflection from the interior of the eye. From the fifth to the seventh day there is a floculent precipitate which forms in the lower part of the anterior chamber, exposing more clearly the iris and the lens and absoption commences. The eye will clear up in 10 or 15 days.

The striking characteristic of the disease, however, is its recurrence again and again until blindness results. The attacks may follow each other at intervals of a month, more or less, but they show no relation to any particular phase of the moon as the name "moonblindness" would lead one to think. The attacks are rather determined by the weather, the health or the food, or some periodicity of the system. From five to seven attacks usually result in blindness in one eye and then the other runs through the same course. In the intervals between the attacks some symptoms remain which usually betray the condition. Even after the first attacks there can usually be seen a bluish ring around the margin of the cornea. The affected eye seems 
smaller than the other, at first from retraction in its socket and later from atrophy. The upper eyelid will have an abrupt bend toward its inner angle from the contraction of the levator muscle. The front of the eye has lost its lustre and the depths are greenish yellow. The ears are alert to compensate for the waning vision.

There is no doubt that some specific germ is responsible for periodic ophthalmia, but it has not been demonstrated. The periodicity of the disease is probably due to the manner of development of the micro-organism, whatever it is, whose generations die out from lack of food in the anterior chamber of the eye and the symptoms subside until the spores develop into virulent organisms again.

Etiology.-The causes may be fundamentally attributed to the soil. Damp clays, marshes and bottoms which have frequently been overflowed are potent causative factors. The damp air and wet climate react upon the animal to produce a lymphatic constitution with an excess of connective tissue, bones and muscle of coarse, open texture, thick skins and gummy legs with profuse long hair. The rank fodders grown on such soils are other causes. Foods act by leading to constipation and undermining the constitution of the animal, giving it a predisposition to any infection. The period of denition and training is a fertile exciting cause. The great majority of victims are from 2-6 years old. If a horse escapes the infection until after he is six years old, he will probably never get it. The irritation about the head during the eruption of teeth, the unwonted bridle and collar, the stimulating grain diet and the close air of the stable all combine to arouse the latent tendency of the disease in the eye. No one of these conditions would cause the attack, but all together have great bearing as predisposing factors at least. It has been alleged that the specific factor is a germ which is harbored in the marshy district, but it has never been found.

Heredity is accepted by horsemen as a most potent factor,cne so strong that intelligent horsemen everywhere refuse to breed from either horse or mare that has once suffered from recurrent ophthalmia. The French Government even refuses ser- 

vice to any mare that has once suffered with her eyes. A consideration of the future of our horses would demand the disuse of all sires that are unlicensed and the refusal of a license to any sire that has suffered from periodic ophthalmia or any other communicable disease.

Treatment.-Treatment is not satisfactory, but the same measures as are useful in external ophthalmia help some in the periodic form. The affection should be treated with purgatives, followed by diuretics and cold applications to the eye. To cause dilatation of the pupil inject a solution of atropin ( $2 \frac{1}{2}$ grains to the ounce) three or four times a day. If the pupil does not contract after a few days, then use calabar bean. Give soft food, keep the animal in a cool place and let him rest. This will ward off the opacity for a time. During the convalescence iodide of potash will help to absorb the deposits, but the animal will go blind in time. Some doctors say that surgical treatment is the right thing and recommend tapping the eye with a fine-bladed knife and squeezing out twenty-five per cent of the aqueous humor. This, at least, would relieve the intraorbital pressure. Following the operation give a dram of iodide of potash three times a day for a month or six weeks.

The prevention of the disease is the great object to be aimed at and this demands the most careful breeding, feeding and housing. The animals should be placed in a high and dry location and kept off the lowlands. The improvement of the land by drainage and cultivation, however, should be the final aim.

SUN STROKE, INSOLATION, COUP DE SOLEIL, THERMIC FEVER, HEAT STROKE, COUP DE CHALEUR, HITZSCHLAG, SONNENSTICH, ICTUS SOLIS, HEAT PROSTRATION, SIRIASIS, OVERHEATING.

Definition.-Under these various names the diseased condition, in which an excessively high temperature, nervous prostration, insensibility, coma and death are the prominent features! occurs." 
Nature.-It is an auto-intoxication with fatigue toxines and toxic decomposition products due to overwork in hot weather.

The heat of warm blooded animals is produced by chemic metabolism, and is regulated by the thermal center in the medulla oblongata. The natural channels through which heat is lost are, according to Helmholtz, $21 / 2 \%$ by the solid and fluid egesta, $5 \mathrm{r} / 4 \%$ by warming inspired air, $143 / 4 \%$ by evaporation of the water carried out by the expired air, and $771 / 2 \%$ by radiation.

Alexander Lambert gives the normal loss of animal heat as follows : $2.6 \%$ by warming food and drink, $2.6 \%$ by warming inspired air, $14.7 \%$ by evaporation and $80.1 \%$ by radiation. These losses are affected by meteorological conditions. In very hot weather the loss caused by warming the food, drink and inspired air is almost nil, and in hot, humid weather, with low barometric pressure, especially if there is no breeze, evaporation and radiation are reduced to a minimum. When the barometer is high the neve tonus is good, the animal feels well and evaporation keeps pace with the perspiration. Then there are no sunstrokes, but when the barometer is low, the weather hot and humid, the animal feels languid, perspiration is profuse, and evaporation is nil sunstrokes are common. Consequently sunstrokes are common on the Atlantic coast, in the region of the great lakes and in tropical climates, but in the Northwestern States they are rare.

Etiology.-A condition of cerebral hyperaemia may come on from excessively active exercise in hot weather, attended by convulsions, syncope, coma and death. This is most often seen in dogs.

In the horse the predisposing causes are old age, overwork or indigestion. The exciting causes are hard work in hot, humid weather with low barometer, insufficient water supply overheating in horses working between other horses in three horse teams or gangs.

Symptomatology.-At first there is profuse perspiration, then the horse begins to pant and the sweat dries up; he now begins to stagger, becomes insensible, goes down, is unable to rise, soon. becomes comatose, the surface of the body is dry and hot, the 

temperature is $109^{\circ}$ to $112^{\circ} \mathrm{F}$., the mucous membranes get livid, the pulse rapid and weak, the respirations at first are rapid, and as coma develops they get slower and finally stertorous. Death in bad cases follows in half an hour to four or five hours.

Prognosis.-With a temperature of $109^{\circ}$ a horse will usually recover if he receives prompt and proper treatment. $110^{\circ}$ or higher will usually prove fatal, unless the treatment is very prompt and the horse is strong.

Special Pathology.-There is a condition of anhydræmia. The blood is thick, slightly if any coagulated, nearly black, with extensive destruction of red corpuscles. The lungs are congested, the right side of the heart is nearly empty, the left side is full of black nearly fluid blood, the brain is congested and the chromophylic plagues or Nissl's bodies are broken up, the liver and kidneys are congested, and the spleen is somewhat enlarged.

Sequelae.-Supersensitiveness to heat that lasts the balance of the season, and sometimes for ever after. This is manifested by panting on slight exertion in hot weather, and staggering from slight cerebral congestion. Some cases become dummies.

Treatment.-Get the patient into the shade as soon as possible, if he is not comatose. Give him a half pint of whisky in as much water or an ounce of aromatic spirits of ammonia in half a pint of cold water. Give a grain of strychnia hypodermatically, and a dose of nitroglycerine if necessary; dram doses of acetanilid will assist in reducing the temperature which must be brought about promptly. To aid in doing this put sacks of ice on the head and neck, and spray the body with cold water by pinching the end of a hose pointed upwards so as to let the water come down on to him like rain. Take his temperature every fifteen minutes and stop the spraying when it is reduced to $103^{\circ}$. If kept up longer it is apt to fall below the normal. In addition to the above, if the initial temperature is $110^{\circ}$ or higher, give rectal injections of cold water with a fountain syringe." During convalescence give nux, aromatic spirits of ammonia, gentian, light diet and long rest.

Prevention.-When horses come in from work hot, sponge them over with cold water and let them dry. Give internally 
aromatic spirits of ammonia and nux and tie the horses outside in the open air. Do not put them into the stable nor feed them till they are well cooled out. During this time give them cold water to drink in small quantities and often. If treated in this way they will be ready for work the next day, but if not they will go out tired next morning and probably collapse before night. When a horse begins to pant collapse is imminent, consequently he should be driven into the shade, unharnessed, sponged off, watered and, when able, driven home.

\section{DEATH BY LIGHTNING.}

Post Mortem findings in death from electricity.

First:-Rigor Mortis is always quick, second or third hour, in 'fifty minutes in one case.

Second:- Points of Penetration show more or less burns. Points of exit show less burning than those of entrance.

Third:-If body is opened immediately the heart is lax, both ventricles full and the auricles pulsating rhythmically. If Post is delayed fifteen minutes or more the left ventricle is sometimes found empty and firmly contracted.

Fourth:- The arteries are contracted to the limit, the blood being forced into the large veins of the trunk and head.

Fifth:-Viscera engorged.

Sixth:-Nervous system presents no change either macroscopical or microscopical. Molecular changes are supposed to occur. but not proven. Brain is sometimes seen with gross destruction of peripheral cortex with intracranial hemorrhage.

Cause of Death.-Paralysis of the heart in ventricular tremulation in case of a low pressure current (syncope), and inhibition of the respiratory center in high pressure current (asphyxia).

Treatment.-Artificial respiration and Faradic current to the precordium, diaphragm and epigastric region. Also bromides and strychnine. 



\section{INDEX.}

\section{PAGE}

Abdominal Glands-Diseases

of $\ldots \ldots \ldots \ldots \ldots \ldots \ldots \ldots 145$

Abscess-Sub-Periosteal .... 229

Abscess-Post Pharyngeal... 55

Active Congestion ........21-22

Acute Bronchitis .......... 57

Acute Farcy ............... 164

Acute Gastric-Impaction..... 109

Acute Indigestion $\ldots \ldots \ldots 106$

Acute Inflammatory Diseases 198

Acute Parenchymatous $\mathrm{Ne}-$

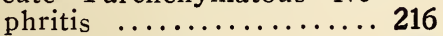

Acute Laryngitis ........ 49

Adhesion ............... 29

Agglutination Test $\ldots \ldots \ldots 168$

Albuminuria ............. 210

Albumin-Test for in Urine.. 210

Amaurosis .............. 257

Anaemia ................ 16

Anaemia-Cerebral ........ 237

Anal Fistula ............ 139

Anasarca ................ 177

Anemia-Infectious ......... 180

Aneurism ............... 207

Angina Pectoris ......... 185

Angiomata ............... 223

Anthrax ................ 153

Anus-Imperforated ........ 140

Apoplexy ..............198-237

Appetite Capricious ........ 111

Arteritis ............... 206

Articular Rheumatism ....... 183

Ascaris Megalocephala ..... 127

Ascites ................ 143

Asphyxia $\ldots \ldots \ldots \ldots \ldots \ldots, 32$

Asthma ................ 68

Atelectasis ............ 57

Atrophy of Bone..........231

Atrophy of Heart........ 203

Auscultation $\ldots \ldots \ldots \ldots \ldots . .40$

Azoturia ................. 190

\section{B}

Bacillus Anthracis ........ 156

Bacillus Malleus ............ 161

Balls-Dust ............... 136

Pastard Strangles ........... PAGE

Bastard Strangles ........ 170

Bean ..................... 218

Big Head .............. 231

Bile-Reabsorption of ....... 150

-Suppression of ...... 150

Biliary Congestion of Liver. 147

Bladder-Eversion of the.... 221 -Rupture of ........ 221

Blind Staggers .......... 236

Blood-The ............. 15

Blood-Composition of the... 16

Blood Diseases ..........36-153

Blood Vessels-Diseases of . . 206

Bloody Flux ............. 144

-Urine ............. 211

Blue Disease .............. 197

Bone-Atrophy of ........ 231

Bones-Discases of .......... 228

Botrymoycosis .......... 174

Bots .................... 118

Bowel Invagination ........ 136

Braxy ................. 155

Bright's Disease .......210-215

Broken Wind ............ 63

Bronchiolitis $\ldots \ldots \ldots \ldots \ldots \ldots .6 \%$

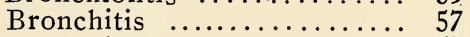

-Acute ............ 57

Bronchocele ............... 227

Broncho-Pneumonia .......57-73

Brown Atrophy of Heart... 203

Buccal Membrane-Congestion of $\ldots \ldots \ldots \ldots \ldots \ldots, 92$

Bull Burnt .............. 249

Burnt Dog ................ 249

Bursatti ................ 172

C

Cachexia ............... 254

Calculi .................... 135

-Salivary $\ldots \ldots \ldots \ldots .98$

Callus $\ldots \ldots \ldots \ldots \ldots \ldots \ldots \ldots 235$

Cancer .................. 223

Capped Hock and Knee.... 225

Capricious Appetite ......... 111

Carbuncle ................ 218

-Contagious ......... 155 


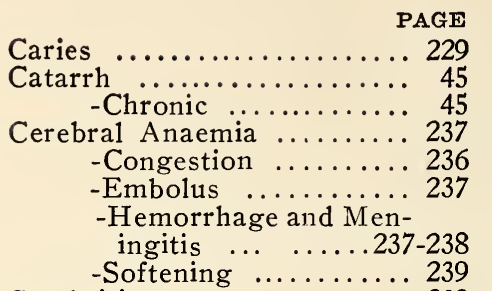

Cerebritis ................ 238

Charbon $\ldots . \ldots \ldots \ldots \ldots \ldots . . .6154$

Choking ................. 101

Chondroma .............. 223

Chorea .................... 246

Chronic Catarrh ........... 45

-Cough ............. 53

-Farcy ............. 163

-Gastritis ........... 115

-Heparitis ........... 149

.Indigestion-Gastric .. 110

-Laryngitis $\ldots \ldots \ldots \ldots . \quad 52$

-Rhinitis ........... 45

-Rheumatism ........ 85

Cirrhosis of Liver.......... 149

Classification of Disease..... 15

Clox-Ante and Post Mortem 27

Coital Exanthema........... 248

Colic-Flatulent .............. 132

-Spasmodic .......... 129

Coma ................. 33

Conditioning of Horse..... 70

Congestion $\ldots . . \ldots \ldots \ldots \ldots .21$

-Active .............21-24

-Pulmonary ......... 59

Congestion-Cerebral ...... 236

Congestion-Hypostatic $\ldots \ldots .21$

-Passive ............ 23

-Physiological ........ 21

Constipation $\ldots \ldots \ldots \ldots \ldots \ldots . .118$

Constitutional Osteo Porosis. 231

Contagious Carbuncle ..... 155

Contagious Pleuro-Pneumonia of Cattle .............. 77

Contagious Pneumonia ..... 77 -Stomatitis .......... 94

Corn Stalk Disease........ 174

Coryza Contagiosa Equorum. 170

Cough-Chronic ............ 53

Cornea Inflammation ........ 256 -Opaque ............ 255

-Ulceration of $\ldots \ldots \ldots 256$

Coughing ................. 41

Coup de Chaleur ........... 261

Coup de Soleil ............ 261

Cow Pox ............... 154
PAGE

Crepitation $\ldots \ldots \ldots \ldots \ldots \ldots \quad 42$

Cribbing ................. 115

Crick Back .............. 242

Cyanosis ................. 197

Cystitis ............... 217

Cysts .................. 225

-Dentigerous ....... 227

-Mucous ......... 227

-Ovarian ........... 227

-Serous ........... 227

D

Death ............... 30

Death by Lightning ....... 264

Decomposition ............ 145

Dentigerous Cysts ....... 227

Detachment of the Retina... 258

Diabetes Insipidus ......... 212 -Mellitus ........... 213

Diagnosis $\ldots \ldots \ldots \ldots \ldots \ldots$ 14-15

Diarrhoea ................. 123

-Acute in the Human.. 126

-Chronic ............ 126

Dicrotic Pulse .............. 19

Digestive System-Diseases of 89

Diphtheria ............ 56

Diseases-Acute Inflammatory. 198

Disease-Classification of.... 15

Disease in General..........

Rational Treatment of. 36

Diseases-Constitutional $\quad \ldots . .153$

Diseases of Abdominal Glands 145

Diseases of Blood Vessels... 206

Diseases of the Circulatory

System .............. 195

Diseases of the Digestive System .............. 89

Diseases of the Mouth....... 92

Diseases of the Nervous Sys-

tem ............... 236

Diseases of the Oesophagus. 100

Diseases of the Organs of Special Sense .......... 253

Diseases of the Reproductive System .............. 247

Diseases of the Stomach.... 105

Diseases of the Throat.... 99

Diseases of the Urinary System ................. 208

Diseases-Respiratory ....... 41

Disinfection of Stables...... 166

Disorganization .......... 30

Dourine ............... 248

Dropsy of the Ovaries...... 250 


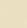



PAGE

Dropsy of the Womb..... 250

Druse ........................... 170

Dry Gangrene .......... 25

Dummy ................239

Dust Balls ............. 136

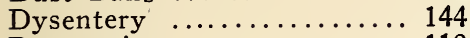

Dyspepsia ................ 110

Dyspnoea ............. 42

Dysuria ............... 218

$\mathbf{E}$

Ecchymosis ........... 23

Ectopia Cordis ........... 206

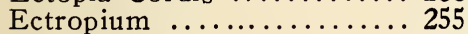

Elephantiasis ............ 187

Embolism .............26-207

Embolus-Cerebral .......... 237

Emesis .................. 105

Emphysema-Pulmonary ....6 63

Empyema ............. 83

Endocarditis ........... 200

Endothelioma ........... 222

Engorgement of Stomach.. 106

Enteritis .............. 140

Entropium ............. 254

Enuresis .............. 220

Ephemeral Fever .......... 37

Epitheliomata ............ 223

Epizootic Lymphangitis .... 189

Equine Syphilis .......... 248

Eructations ............... 107

Etiology ..............13-14

Eversion of the Rectum.... 137

\section{F}

Fabricula ............. 37

Fainting ................... 198

Farcy ................. 161

Fatty Degeneration ........ 205

Fever-Ephemeral ......... 37

-Mixture ........... 39

-Putrid ........... 155

-Rational Treatment of 37

-Simple ........... 37

Fibrinous Stage ........... 59

Fibroma .............2222-224

Filaria Oculi ............. 256

Fistula-Anal ............. 139

-Intestinal .......... 134

-Salivary ............ 97

Flatulence-Gastric ........... 106

Flatulent Colic .......... 132
PAGE

Flooding ............. 212

Flukes ................ 152

Flux-Bloody ............ 144

Foetal Lung ............57-60

Friction Sounds ......... 42

Fungus-Bleeding ......... 254

\section{G}

Gangrene ............. 24

Gargle-Iron ............. 53

Gastric Flatulence ........ 106 -Impaction-Acute ..... 109 -Indigestion-Chronic... 110

Gastritis ............. 113

-Chronic .......... 115

Glanders ............... 161

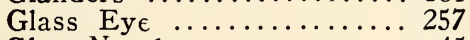

Gleet-Nasal ............. 45

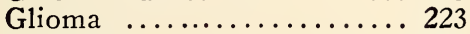

Glossitis .............. 94

Glycosuria ............ 213

Goitre ................ 227

Gourme $\ldots \ldots \ldots \ldots \ldots \ldots \ldots . \ldots 170$

Grunting $\ldots \ldots \ldots \ldots \ldots \ldots, 41$

Gut-Tie .............. 136

Gut-Twist ........... 136

Gutta Serena ........... 257

H

Haematoides Fungus ...... 254

Healing of Bone.......... 235

Heart .................... 203

-Atrophy .......... 203

-Dilatation of ....... 202

-Fatty Degeneration of 205

-Failure-Sudden ...... 31

-Hypertrophy ....... 203

-Inflammation of Serous

Sack of .......... 198

-Inflammation of Valves

of ............... 199

-Muscle Inflammation. 203

-Obesity of ........205

-Polypi ........... 206

-Rupture of ........ 206

-Tumors ..........206

Heat Stroke ............ 261

Heaves-Confirmed and In-

cipient ..........6.63-66-68

Hematuria .............2 211

Hemiplegia ............. 241

Hemophilia ............16-23

Hemorrhage by Rhexis..... 142

Hemorrhage-Internal and $\mathrm{Ex}$ ternal 
Hemorrhagic Infarction .... 69 Hemorrhoids ............ 139 Hepatitis ................. 148 -Chronic .......... 149

Histioid Tumors ........ 222

Hitzschlag ............. 261

Horse Sickness .......... 155

How to Kill a Horse....... 33

Hydrocephalus ........... 241

Hydrometra ............ 251

Hydrophobia ........... 158

Hydrothorax ........... 83

Hygiene ...............14-15

Hyperemia ............. 21

Hypertrophy of Heart....203-204

Hypostatic Congestion ....21-72

Hysteria .............. 252

\section{I}

Icterus $\ldots \ldots \ldots \ldots \ldots \ldots 146$

Ictus Solis ............. 261

Imperforated Anus ........ 140

Inanition $\ldots \ldots \ldots \ldots \ldots \ldots \ldots .25$

Indigestion $\ldots \ldots \ldots \ldots \ldots \ldots 106$

-Chronic ........... 106

-Subacute .......... 106

Induration $\ldots \ldots \ldots \ldots \ldots \ldots .29$

Infectious Anemia ......... 180

Inflammation ........... 28

-of the Bowels....... 140

-of the Conjunctiva.. 253

-of the Liver ........ 148

Influenza ............... 175

Insolation $. . \ldots \ldots \ldots \ldots \ldots . . .261$

Inspection $\ldots \ldots \ldots \ldots \ldots \ldots .40$

Institutes of Medicine....... 13

Intermittent Pulse ......... 19

Intersitial Pneumonia of

Glanders ............. 77

Intestinal Fistula .......... 134

-Obstructions ........ 135

-Wall Rupture ....... 140

Introduction ............ 12

Intussusception .......... 136

Iron Gargle ............. 53

Irregular Pulse .......... 19

Irritant ................ 27

Ischuria $\ldots \ldots \ldots \ldots \ldots \ldots . \ldots 219$

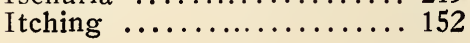

Jaundice $\ldots \ldots \ldots \ldots \ldots \ldots 146$

$\mathrm{K}$

Keratitis .............. 256

Kidneys-Hyperaemia of the. 214
Kidneys-Inflammation $\ldots \ldots .215$

Killing of Horse ......... 33

\section{L}

Lampas ............. 92

Laryngitis ............ 48

-Acute ........... 49

-Chronic ........... 52

-Necrotic .......... 48

Laryngo-Pharyngitis ...... 48

Leiomyoma ............ 228

Leucoma ............... 255

Leucorrhoea .............. 252

Lipoma .............. 223

Liver-Active-Passive and

Biliary Congestion ........ 146

-Cirrhosis of the...... 149

-Congestion of ......... 146

-Fatty Degeneration of 150

-Inflammation of the.. 148

-Diseases of the..... 145

Locomotor A taxia ........ 242

Loodiana Disease ......... 155

Lymphadenitis ........... 186

Lymphadenoma ............ 152

Lymphangitis ............ 186

-Anaemic ........... 190

-Epizootic ........... 186

-Mycotic ........... 186

-Septic ............ 190

-Ulcerative .......... 186

Lumbago .............. 183-184

\section{M}

Mad Staggers .......... 239

Malarial Fever ........... 180

Malignant Pox ........... 247

-Pustule ............ 156

Mallein Test .............164-167

Malleus-Baccilus ........... 161

Massage of the Bowels..... 123

Masturbation ............. 253

Mechanical Engorgement ... 69

Meconium-dry ............ 120

Megrims ............... 236

Meningitis-Cerebral ....... 230

Mensuration ............ 40

Metastatic Rheumatism ..... 183

Micturition-Profuse ......... 212

Milzbrand .............. 155

Moist Gangrene ........... 24

Monday Morning Sickness.. 187

Moonblindness ........... 259

Morbid Anatomy ........... 14

Morve-La .............. 161 



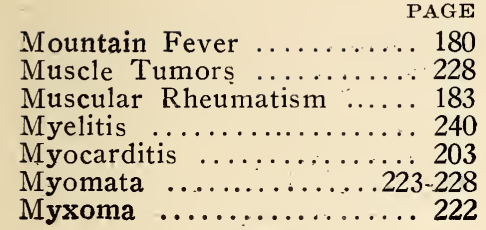

\section{$\mathbf{N}$}

Nasal Gleet ............. 45

Nausea $\ldots \ldots \ldots \ldots \ldots \ldots \ldots . .6107$

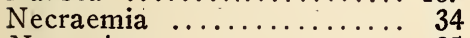

Necrosis ................ 25 -of Bone ............ 230

Necrotic Laryngitis ....... 48

Negri Bodies ............... 159

Nematoda $\ldots \ldots \ldots \ldots \ldots \ldots, 128$

Nephritis ............... 215

Nervous System-Disease of. 236

Neuralgia $\ldots \ldots \ldots \ldots \ldots \ldots, 183-188$

Neuroma $\ldots \ldots \ldots \ldots \ldots \ldots \ldots .225$

Neuromata .............. 223

Nicolaier Bacillus ............ 243

Noncontagious Pneumonia .. 73

Noname Disease ......... 180

Normal Respiration of Horse 37

Nosology ...............13-35

Nymphomania $\ldots . \ldots \ldots \ldots 252$

\section{0}

Obesity of the Heart...... 205

Obstructions-Intestinal .... 135

Occlusion .................. 60

Oedema ................... 22

Oedema Glottidis ........... 48

Oesophagismus ............ 101

Oesophagitis ............. 100

Oesophagus-Organic Diseases of $\ldots . . . \ldots \ldots \ldots . . . . . .104$

Onanism .................... 253

Opaque Cornea ........... 256

Ophthalmia Periodic ...... 259

-Recurrent ........... 259

-Simple ............. 253

-Specific ............ 259

Organic Diseases of the Oesophagus ............... 104

Organs of Special Sense-Diseases of ............... 253

Organization .............. 30

Orthotonos ............... 244

Ossium-Fragilitis .......... 233

Osteitis ................... 228

Osteoclasts .................. 235
PAGE

Osteosclerosis ........... 229

Osteoma ................. 223

Osteomalacia $\ldots . \ldots \ldots \ldots . . .232$

Osteomyelitis ............ 228

Osteoporosis .............. 229

-Constitutional ...... 231

Ovaries-Dropsy of the..... 250

Overheating $\ldots \ldots \ldots \ldots \ldots . .261$

Oxaluria ................. 213

Oxyuris Curvula .......... 128

Ozena $. . . \ldots \ldots \ldots \ldots \ldots \ldots . \ldots 45$

\section{$P$}

Palatine Artery Hemorrhage 92

Palpation .................. 40

Palpitation $\ldots{ }_{2} \ldots \ldots \ldots \ldots \ldots . .6105$

Palsy ................... 241

Pancreas-Fatty Degeneration 153

Paracentesis Thoracis ...... 86

Paralysis ............... 240 -of Throat ........... 54

Paraphimosis ........... 250

Paraplegia ............... 241

Paresis ................ 240

Parotid Gland Inflammation. 95

Parotiditis ............... 95

Pasty Mouth .............. 124

Pathogenesis ............. 13

Pathogeny ..............13-14

Pathology .............. 13

-General ............ 13

Percussion ............... 40

Pericarditis .............. 198

Periodic Ophthalmia ....... 259

Periostitis ............... 229

Peritonitis ............... 143

Pernicious Anemia ........ 180

Petaechial Typhus .......... 155

Petechiae ................. 23

Pharyngitis ................ 99

Phlebitis ................ 207

Phlyctena $\ldots . \ldots \ldots \ldots \ldots \ldots, \ldots, 48$

Phymosis $\ldots . . \ldots \ldots \ldots \ldots \ldots . .250$

Piles-Bleeding ............. 139

Pin Worms .............. 128

Plains Paralysis .......... 180

Plethora ............... 16

Pleural Adhesions ........... 85

Pleurisy ................. 81

Pleuro-Pneumonia ........ 73

Pleuro-Pneumonia of Cattle. 77

Pneumonia ............. 72

Pneumonia-Contagious ..... 77 -Broncho ...........57-73 -of Glanders-Interstitial 77 
PAGE

Pneumothorax .......... 88

-Record of a Case of. . 88

Polypi of the Heart ....... 206

Polyuria ...............111-211

Post Mortem Digestion..... 118

Post Pharyngeal Abscess.... 55

Pox-Simple and Malig-

nant .............247-248

Prognosis ............... 14

Protozoon $\ldots \ldots \ldots \ldots \ldots \ldots \ldots . \ldots 153$

Ptyalism ................... 96

Pulmonary Apoplexy ...... 69

-Congestion .........6 69

-Emphysema ....... 63

Pulsating Tumor ......... 207

Pulse.................. 17

-Dicrotic ........... 19

-Frequent and Infrequent $. . \ldots \ldots \ldots \ldots, 18$

-Hard and Soft....... 19

-Intermittent ........ 19

-Irregular .......... 19

-Large and Small .... 18

-Quick and Slow...... 18

-Venous ............. 19

Purpura Hemorrhagica ..... 177

Pustule-Malignant ......... 156

Putrid Fever ............ 155

Pyogenic Fever .......... 170

Q

Quinsy

\section{4}

$\mathbf{R}$

Rabies .............. 158

Rachitis .............. 233

Rales ................ 42

Ranula ................. 227

Rational Treatment of Disease in General ......... 36

Rational Treatment of Fever 37

Rectum-Eversion of the.... 137

-Tenesmus of ....... 144

Recurrent Ophthalmia ..... 259

Renal Calculus ........... 217

-Congestion .......... 214

Reproductive System

-Diseases of the...... 247

Respiratory Diseases ...... 41

Results of Active Congestion 24

Retina-Detachment of the... 258

Rhabdomyoma ........... 228

Rhexis-Hemorrhage by ..... 142

Rheumatic Fever ......... 182
PAGE

Rheumatism ........... 182

Rhinitis ...............42-47

Rickets ................. 233

Roaring .............42-52

Rotzkrankheit ............ 161

Rupture of Heart........ 206 -of Intestinal Wall.... 140 -of Stomach ........ 116

\section{S}

Saccharomyces Farciminosus 189

Sales Stable Fever......... 86

Salivary Calculi ............ 98

-Fistula ........... 97

Sarcoma .............. 222

Satyriasis $\ldots \ldots \ldots \ldots \ldots \ldots \ldots 252$

Scarlatina ............... 179

Sciatica .................183-184

Scirrhous Cord ............ 227

Scirrhous Cancer .......... 223

Sclerosis of the Cord....... 242

Sclerostoma Equinum ....... 127

Scouring ................ 127

Semeiology ............ 13

Septic Lymphangitis ....... 190

Sequestrum ............ 230

Shakes ....................... 187

Shipping Fever ........ 77

Shivers ............... 246

Shot of Grease........... 187

Sibilant Sounds ........... 59

Simple Pox ............ 247

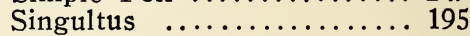

Siriasis $\ldots \ldots \ldots \ldots \ldots \ldots \ldots 261$

Sitfast $\ldots \ldots \ldots \ldots \ldots \ldots \ldots .25$

Sneezing $\ldots \ldots \ldots \ldots \ldots \ldots \ldots, 42$

Snoring $\ldots \ldots \ldots \ldots \ldots \ldots \ldots, 42$

Sonnenstich ............... 261

Sore Throat ........... 48

Spasmodic Colic ........... 129

Specific Ophthalmia ........ 209

Sphacelation .............. 48

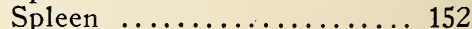

Splenic Fever ........... 155

Staggers-Stomach ........ 109

Staphyloma ............. 258

Sthenic Syncope .......... 31

Stomach-Diseases of ........ 105

-Engorgement ....... 106

-Rupture of ........ 116

-Staggers ........... 109

Stomatitis $\ldots \ldots \ldots \ldots \ldots \ldots . \ldots 9$

Stomatitis Pustulosa Contagiosa

94-155 
. 

Strausstest ............. 167

Stricture $\ldots \ldots \ldots \ldots \ldots \ldots \ldots . .136$

Strangles $\ldots \ldots \ldots \ldots \ldots \ldots \ldots . \ldots 170$

Strangury ................. 220

Streptococcus Equi ........ 171

Strongylus Armatus ......... 127

Subaçute Indigestion...$\ldots .106$

Subacute Rhinitis .......... 45

Succussion ............... 41

Sun Stroke .............. 261

Superpurgation $\ldots \ldots \ldots \ldots \ldots .125$

Suppuration ............... 59

Surra ................... 174

Swamp Fever ..........175-180

Symptomatology .........13-14

Syncope $\ldots . \ldots \ldots \ldots \ldots \ldots . . .198$

-Asthenic .......... 31

-Sthenic ............. 31

Syphilis Equine ............ 248

\section{$\mathbf{T}$}

Tabes Dorsalis ........... 242

Tapping $\ldots \ldots \ldots \ldots \ldots \ldots \ldots . . .133$

Teeth-bearing Cysts $. \ldots \ldots . .227$

Temperature $\ldots \ldots \ldots \ldots \ldots .20$

Tenesmus .............. 217 -of Rectum ......... 144

Teratomata ............. 223

Tetanus $\ldots \ldots \ldots \ldots \ldots \ldots \ldots 243$

Therapeutics $\ldots \ldots \ldots \ldots \ldots . .614$

Thermic Fever ............ 261

Thirst-Excessive .......... 111

Throat-Diseases of $\ldots \ldots \ldots \ldots 99$

-Paralysis of $\ldots \ldots \ldots .54$

Thrombosis ............26.207

Thrush-Baby's ........... 93

Thumps ............... 93

Torti Colis .............. 185

Tracheotomy $\ldots . . . \ldots \ldots \ldots . .651$

Trismus ............... 244

Trypanosoma Equiperdum .. 248

Trypanosoma Evansi ....... 175

Trypanosomiasis $\ldots \ldots \ldots \ldots .6175$

Tubular Breathing ........... 75

Tumor-Pulsating ........... 207

Tumors of the Heart..... 206

Tumors ................. 221

Tympanitis $\ldots \ldots \ldots \ldots \ldots \ldots . \ldots 132$
Tympanitis of the Rumen... 102 Typhoid Fever .........144-180

\section{U}

Ulceration of the Cornea.... 255 Ulcerative Lymphangitis ... 186 Unknown Disease- 1 'he ..... 180 Unthriftiness ............ 111 Uraemia ................ 193 Urethritis ............221-249 Urinary System-Diseases of. 208 Urine-Bloody ............. 211 -Incontinence .......220 -Sugar in .......... 213 -Suppression of the... 219 Uterine Haemorrhage ...... 198

\section{V}

Vaccina ................. 154

Vaccination ................ 154

Valvular Disease .......... 202

Valvulitis ................ 200

Varicose Veins ............ 208

Variola .................. 154

Varix ..................... 208

Veins-Inflammation of ...... 207 -Dilated ............ 208

-Varicose ........... 208

Venous Pulse .............. 19

Verminous Cyst ............ 128

Vertigo ................. 236

Volvulus ................. 136

Vomition .............105-116

\section{W}

Weed .................. 187

Wheezing ..............42-52-69

Whistling $\ldots \ldots \ldots \ldots \ldots \ldots \ldots .42-52$

Wind Galls .............. 225

Wind Sucking ............ 115

Womb-Dropsy of the...... 251

Woolsorter's Disease ....... 155

Worms in Colic............. 128

-Armed .............. 129

-Common Pin ....... 129

-Large Round ........ 129 

$y=x$

$$
350 \text { ust }
$$




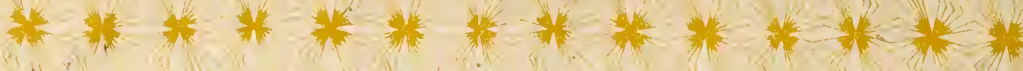

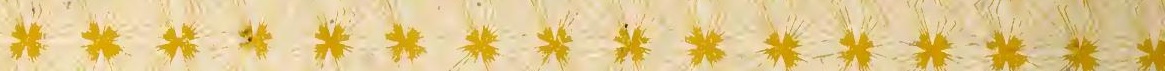
*

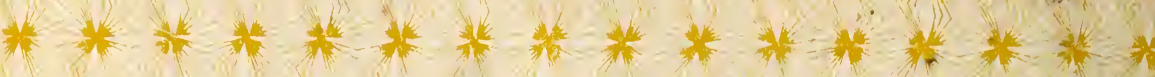
to

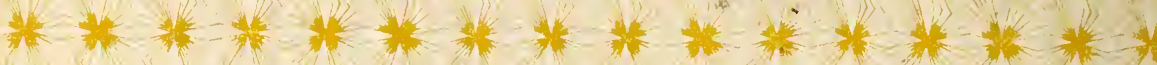

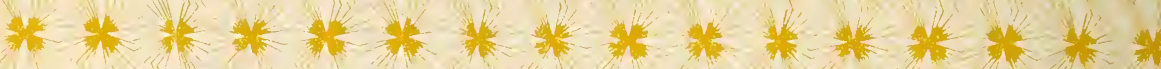

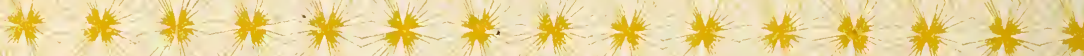
H. *t A I

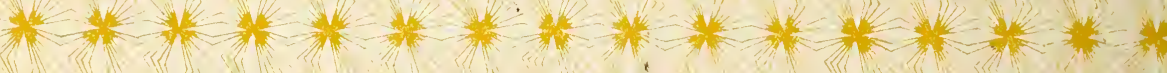

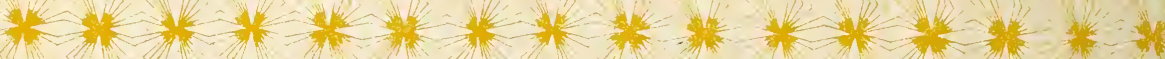

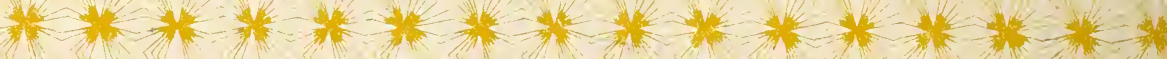

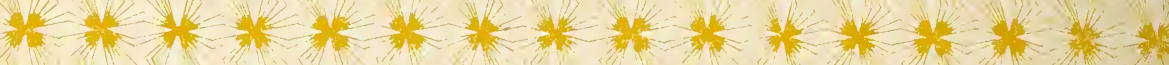

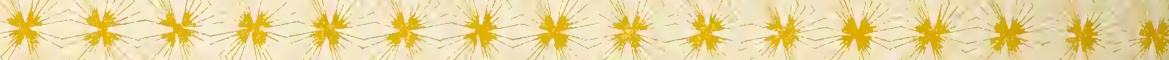

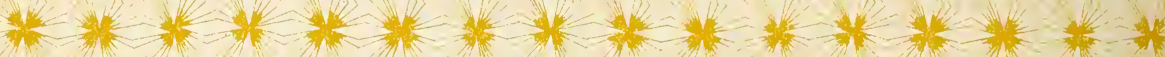
H

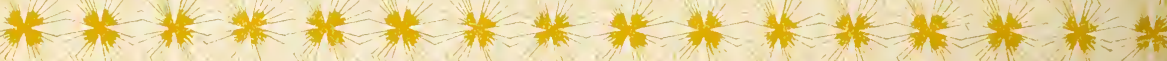

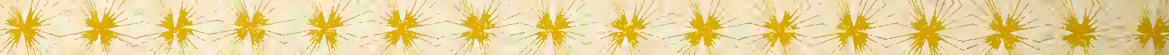
N

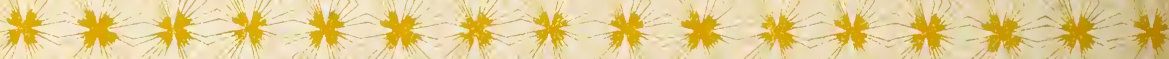

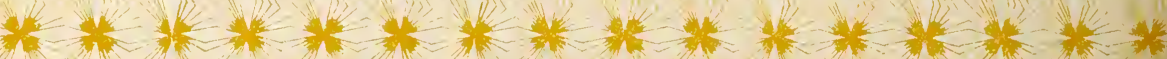
* औ N

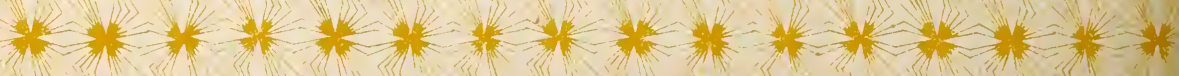

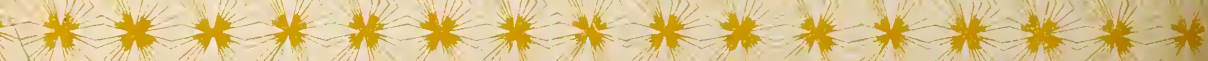




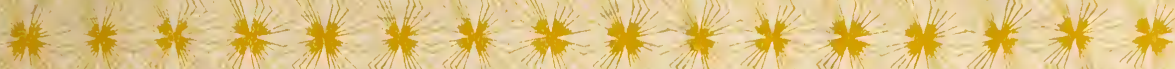

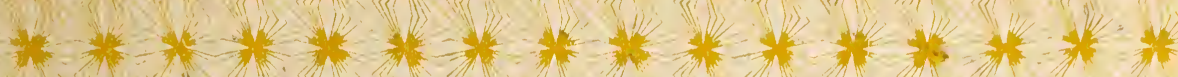

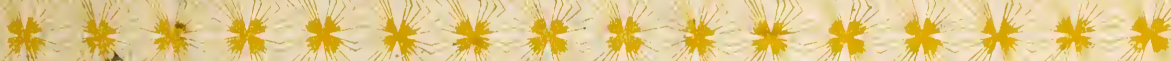

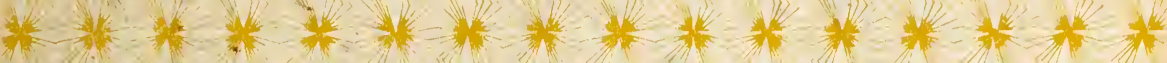

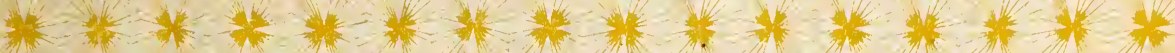

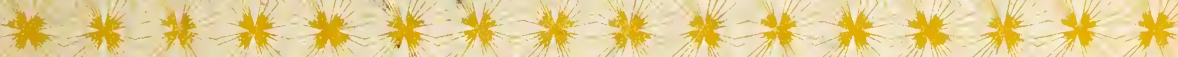

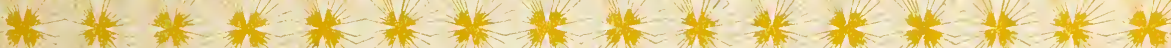

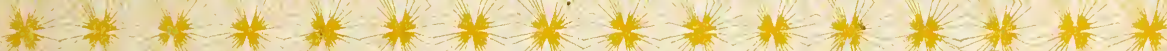

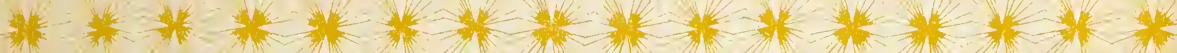

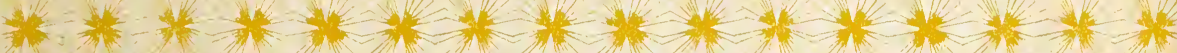

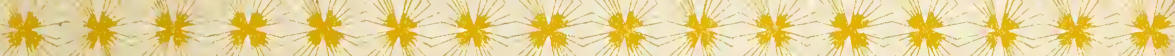

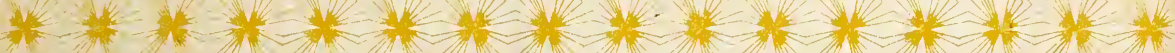

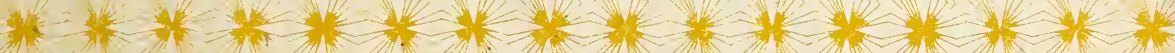

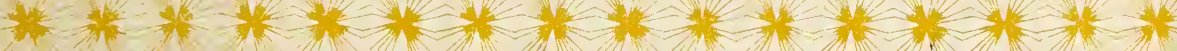

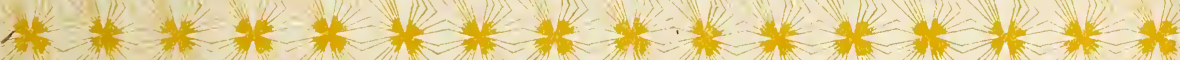

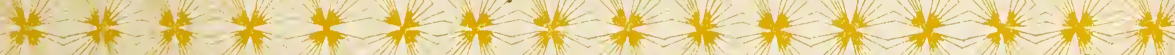

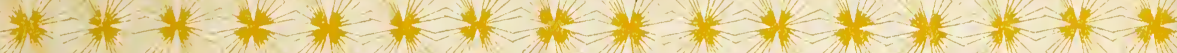

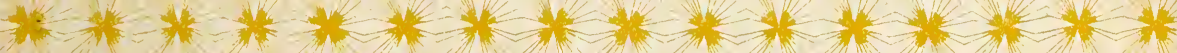
3 (1)

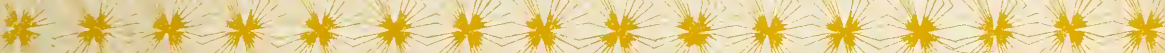

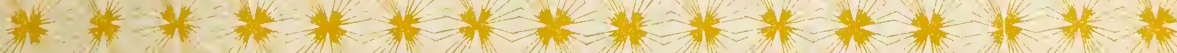

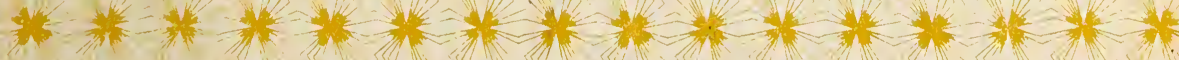

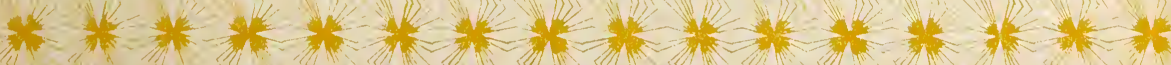

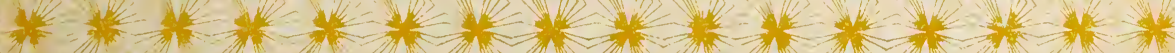

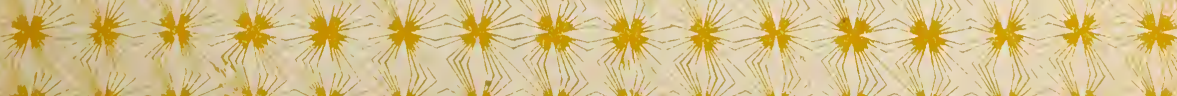

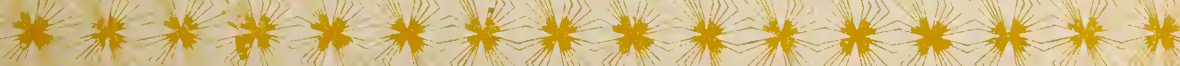


